\section{THEORETICAL STUDIES ON THE ROLE OF TRANSITION IN DETERMINING FRICTION AND HEAT TRANSFER IN SMOOTH AND \\ ROUGH PASSAGES}

FINAL REPORT

FHMT REPORT No. 005

\author{
N. T. Obot and E. B. Esen \\ Fluid Mechanics, Heat and Mass Transfer Laboratory \\ Clarkson University \\ Potsdam, New York 13676-5705 \\ T. J. Rabas \\ Energy Systems Division \\ Argonne National Laboratory \\ Argonne, Illinois 60439
}

APRIL 1990

Prepared for

THE U.S. DEPARTMENT OF ENERGY AGREEMENT NO. DE-FG02-89CE90029.A000 


\section{DISCLAIMER}

This report was prepared as an account of work sponsored by an agency of the United States Government. Neither the United States Government nor any agency Thereof, nor any of their employees, makes any warranty, express or implied, or assumes any legal liability or responsibility for the accuracy, completeness, or usefulness of any information, apparatus, product, or process disclosed, or represents that its use would not infringe privately owned rights. Reference herein to any specific commercial product, process, or service by trade name, trademark, manufacturer, or otherwise does not necessarily constitute or imply its endorsement, recommendation, or favoring by the United States Government or any agency thereof. The views and opinions of authors expressed herein do not necessarily state or reflect those of the United States Government or any agency thereof. 


\section{DISCLAIMER}

Portions of this document may be illegible in electronic image products. Images are produced from the best available original document. 


\begin{abstract}
It has been established that transition determines the attainable friction and heat transfer in smooth and rough passages. According to the proposed law of corresponding states for friction, different types of roughness exhibit the same general behavior for friction at the same reduced conditions. This is also true of different types of smooth passages. It has been fully demonstrated that, in rough passages, the marked increases in friction factor are intimately associated with early transition and that, under reduced similarity conditions, the friction factors are considerably lower than those deduced from the familiar $f$ vs. Re plots.

For all smooth or rough passages, the simple rule for heat transfer amounts to this: the lower the critical Reynolds number for transition, the greater the value for the average heat transfer coefficient. Consequently, for a given Reynolds number based on the hydraulic diameter, triangular passages can be expected to give heat transfer coefficients that are significantly higher than for circular, rectangular or annular tubes. For smooth and enhanced passages of complex shapes, it appears that heat transfer coefficients can be calculated accurately from the smooth circular tube relations, provided the critical Reynolds number is known.
\end{abstract}


Page No.

ABSTRACT

ii

Table of Contents

iii

List of Figures

iv

List of Tables

vi

Nomenclature

vii

1. DNTRODUCTION

2. ANALYSES OF FRICTION IN FLOW PASSAGES

2.1 Smooth Passages

2

2.2 Rough Passages

3. ANALYSES OF HEAT TRANSFER IN FLOW PASSAGES

3.1 Smooth Passages

3.2 Rough Passages

3.2.1 Transition-Based Similarity Parameter Vs. Roughness Reynolds Number

4. FLUID HEATING VERSUS COOLING

5. DISCUSSION

56

6. CONCLUSIONS AND RECOMMENDATIONS

60

6.1 Conclusions

6.2 Recommendations

ACKNOWLEDGEMENTS

62

REFERENCES

64

APPENDIX

71 


\section{List of Figures}

Fig. No.

Page No.

1 Friction factor for circular and noncircular passages. 4

2 Effect of $e / D$ on resistance coefficient according to Koch (1960). 8

3 Variation of $\psi_{R}\left(=R e_{c, r} / R e_{c, a}\right)$ with $e / D . \quad 11$

$4 \quad$ Variation of $\psi_{f}\left(=f_{c, r} / f_{c, a}\right)$ with $e / D$. 12

$5 \quad$ Variation of $\psi_{f}$ with $\psi_{R}$. 13

6 Similarity plot of $f_{m}$ vs. $R e_{m}$ using Koch's (1960) data. 17

$7 \quad$ Plot of $f_{m}$ vs. $R e_{m}$ using Nunner's (1956) data. 18

8 Nikuradse's sand-grain roughness vs. Koch's ring inserts. 20

$9 \quad$ Nusselt number $\left(N u_{m}\right)$ vs. $R e_{m}$ for smooth annuli. 24

10 Nusselt number $\left(N u_{m}\right)$ vs. $R e_{m}$ for smooth annuli. 25

11 Effect of $e / D$ on Nusselt number according to Koch (1960). 28

12 Nusselt number $\left(N u_{m}\right)$ vs. $R e_{m}$ using Koch's (1960) data. 30

$13 N u_{m}$ vs. $R e_{m}$ using Nunner's (1956) data. 32

$14 N u_{m}$ vs. $R e_{m}$ using data of Blackwelder and Kreith (1970). 33

$15\left(N u_{m} / \mathrm{Pr}^{0.4}\right)$ vs. $\operatorname{Re}_{m}$ using data of Knudsen and Katz (1950). 34

16 Maximum enhancement factor, $\phi_{h, \max }$, vs. $\psi_{R}$. 37

$17 \quad$ Variation of $\phi_{h, \max }$ with $\phi_{f}\left(=f_{m} / f_{m, s}\right)$. 38

$18 \quad$ Plot of $\left(\phi_{h, \max } / \phi_{f}\right)$ vs. $\psi_{R}$. $\quad 39$

19 Stanton number ratio versus $R e_{m}$. 45

20 Stanton number ratio versus $R e_{\tau m}$. 46

21 Stanton number ratio versus $e_{m}{ }^{+}$. 47

22 Stanton number ratio versus $e^{+}$. 48 
Fig. No.

Page No.

23 Stanton number ratio versus $R e_{m}$. 50

24 Stanton number ratio versus $R e_{\tau m}$. 51

$25 \quad$ Generalized plot of $\psi_{f}$ vs. $\psi_{R}$ for smooth and rough passages. $\quad 57$ 


\section{List of Tables}

Table No.

Page No.

1 Summary of critical data for enhanced passages.

6

$\begin{array}{lll}\text { A.1 Data of Allen and Grunberg (1937). } & 73\end{array}$

A.2 Data of Cornish (1928). 74

A.3 Data of Carlson (1959). 76

$\begin{array}{lll}\text { A.4 Data of Hartnett et al. (1959). } & 79\end{array}$

$\begin{array}{lll}\text { A.5 Data of Jones (1976). } & 81\end{array}$

A.6 Data of Koch and Feind (1958). 83

$\begin{array}{lll}\text { A.7 Data of Koch (1960). } & 91\end{array}$

$\begin{array}{lll}\text { A.8 Data of Nunner (1956). } & 107\end{array}$

$\begin{array}{lll}\text { A.9 Data of Nikuradse (1933). } & 149\end{array}$

A.10 Data of Blackwelder and Kreith (1970). 156

A.11 Data of Knudsen and Katz (1950). 160

A.12 Data of Watkinson et al. (1974). 165 


\section{Nomenclature}

$$
\begin{aligned}
& A=\text { cross section flow area, } \mathrm{m}^{2} \\
& a_{r} \quad=\text { aspect ratio, } w / s \\
& b=\text { base of triangular duct, } \mathrm{m} \\
& c_{p}=\text { specific heat, } \mathrm{J} / \mathrm{kg}^{\circ} \mathrm{C} \\
& D, D_{e}=\text { maximum internal diameter, equivalent (hydraulic) diameter, } \mathrm{m} \\
& d_{i}, d_{o}=\text { inner, outer diameter of annular space, } \mathrm{m} \\
& e=\text { roughness height, } \mathrm{m} \\
& e^{+} \quad=\text { roughness Reynolds number, equation (7) } \\
& e_{m}^{+} \quad=\text { modified roughness Reynolds number, equation (8) } \\
& f \quad=\text { Fanning friction factor } \\
& f_{m} \quad=\text { reduced friction factor, equation (2) } \\
& f_{a}=\text { friction factor for arbitrary condition, equation (2) } \\
& h \quad=\text { height of triangular duct, } \mathrm{m} \\
& \bar{h}=\text { heat transfer coefficient, } \mathrm{W} / \mathrm{m}^{2}{ }^{\circ} \mathrm{C} \\
& k=\text { fluid thermal conductivity, } \mathrm{W} / \mathrm{m}^{\circ} \mathrm{C} \\
& L_{e} \quad=\text { entrance length, } \mathrm{m} \\
& N u=\text { Nusselt number, } \bar{h} D / k \text { or } \bar{h} D_{e} / k \\
& N u_{m}=\text { Nusselt number associated with } R e_{m} \\
& \operatorname{Pr} \quad=\text { Prandtl number, } \nu / \alpha \\
& P_{w} \quad=\text { wetted perimeter, } \mathrm{m} \\
& p \quad=\text { roughness pitch, } m \\
& R e \quad=\text { Reynolds number } \\
& R e_{a}=\text { Reynolds number for arbitrary condition, equation (2) } \\
& R e_{m}=\text { similarity parameter or reduced Reynolds number, equation (2) } \\
& R e_{\tau m}=\text { friction Reynolds number, equation (10) } \\
& s=\text { channel spacing, } m
\end{aligned}
$$




$$
\begin{array}{ll}
S t & =\text { Stanton number, } h / \rho c_{p} \bar{V} \\
T_{f}, T & =\text { fluid, temperature, }{ }^{\circ} \mathrm{C} \\
U & =\text { velocity, } \mathrm{m} / \mathrm{s} \\
U_{\tau} & =\text { friction velocity, }\left(\tau_{w} / \rho\right)^{1 / 2}, \mathrm{~m} / \mathrm{s} \\
\bar{V} & =\text { average flow velocity, } \mathrm{m} / \mathrm{s} \\
w & =\text { channel width, } \mathrm{m} \\
x & =\text { coordinate in flow direction } \\
y & =\text { coordinate normal to flow } \\
\alpha & =\text { apex angle, deg.; thermal diffusivity, } \mathrm{m}^{2} / \mathrm{s} \\
\beta & =\text { diameter ratio, } d_{i} / d_{o} \\
\mu & =\text { fluid viscosity, } \mathrm{Pa} \mathrm{s} \\
\nu & =\text { kinematic viscosity, } \mathrm{m}^{2} / \mathrm{s} \\
\rho & =\text { fluid density, } \mathrm{kg} / \mathrm{m}^{3} \\
\tau_{w} & =\text { wall shear stress, } \mathrm{N} / \mathrm{m}^{2} \\
\phi_{f} & =\text { friction factor ratio, } f_{m} / f_{m, s} \\
\phi_{h} & =\text { ratio of heat transfer coefficient, } N u_{r} / N u_{s} \\
\psi_{f} & =\text { ratio of critical } f, \text { equation }(1) \\
\psi_{R} \quad=\text { ratio of critical } R e, \text { equation }(1)
\end{array}
$$

\section{Additional Subscripts}

$$
\begin{aligned}
& c, r=\text { critical parameters for reference condition } \\
& c, a=\text { critical parameters for arbitrary condition } \\
& m a x=\text { maximum value } \\
& m, s ; s=\text { smooth duct } \\
& m, c=\text { critical value of similarity parameter } \\
& r \quad=\text { rough condition } \\
& w \quad=\text { value at the wall }
\end{aligned}
$$




\section{INTRODUCTION}

Transition, a process whereby the motion of a fluid changes from laminar to turbulent flow, was successfully traced by Reynolds (1883) over a century ago. Although this remarkable process occurs naturally when the critical velocity and pressure attain some threshold values, it is also well known that its onset can be delayed or accelerated markedly. For any particular condition, be it of a natural origin or one in which the process is effected by artificial means, the most notable characteristic of transition is the marked change in the resistance to flow. Despite the extensive studies on transition (Morkovin, 1958; Dryden, 1953), and even though it is of considerable importance in convective heat transfer, the underlying physics of this phenomenon as well as its implications have eluded complete understanding.

The connection between fluid friction and heat transfer was also postulated by Reynolds in 1874, even before the very exhaustive studies on transition and the laws of resistance, and the resulting relation is generally referred to as the Reynolds analogy. Thereafter, the problem of friction and heat transfer analogy was studied by very eminent researchers (Stanton, 1897, 1913; Lanchester, 1913; Taylor, 1916; Prandt1, 1928; to mention but a few). An overview of the many contributions on the subject, prior to 1933, is given by Colburn (1933). Rather surprisingly, although there are clear indiciations that transition can have very profound effects on surface friction, it does not appear that hitherto any systematic investigations have been made to establish connections between transition and heat transfer, or a global mechanism for heat transfer enhancement. Consequently, the origin of the differences among the various results obtained on smooth passages or of the anomaly between the magnitudes of the increase in frictional pressure coefficients and heat transfer coefficients in the presence of roughness has been open to conjecture. For example, in connection with the latter, Owens and Thomson (1963) 
argued that the rate of heat transfer is controlled by a purely molecular property of the fluid, its thermal conductivity, whereas the shear stress, augmented by roughness, is transmitted to the surface as form drag on the individual elements. The difficulty with this explanation is that it provides no insight on the physical phenomenon involved.

The work described in this paper has its origin in a thorough re-analysis of the literature on friction in smooth circular and noncircular passages which resulted in the formulation of the critical friction method or the frictional law of corresponding states, as well as the fundamental requirements for flow similarity (Obot, 1988). However, it became quite evident that the implications of transition to friction or heat transfer are far too important and more numerous than can be inferred from much that has been written on the subject. It was with this understanding that the aforementioned study was extended to include friction and heat transfer in smooth and rough passages. As with the earlier study, this analysis was carried out using existing literature data. However, in sharp contrast to the manual extraction of much of the data used therein, the data for this study were extracted digitally. A complete tabulation of all data is given in the Appendix of this report.

\section{ANALYSES OF FRICTION IN FLOW PASSAGES}

This treatment of the influence of transition on friction in flow passages is divided into two sections. The first gives an overview of the effects for flow in smooth channels, while a more extensive evaluation of the situation with different types of enhanced tubes is presented in Section 2.2.

\subsection{Smooth Passages.}

In a recent paper (Obot, 1988), the critical friction law (or the law of corresponding states), which asserts that flows having the same critical friction factor and critical 
Reynolds number are dynamically similar to one another, was formulated and successfully applied to smooth tubes of complex shapes. The simple mathematical relations that are needed to effect such an analysis are given by equations (1) and (2):

$$
\begin{aligned}
& \psi_{R}=R e_{c, r} / R e_{c, a} \quad ; \quad \psi_{f}=f_{c, r} / f_{c, a} \\
& R e_{m}=\psi_{R} R e_{a} \quad ; \quad f_{m}=\psi_{f} f_{a}
\end{aligned}
$$

In equation (1), $R e_{c, r}$ and $f_{c, r}$ are the reference values for the critical Reynolds number and critical friction factor, while the corresponding critical parameters for an arbitrary condition are $R e_{c, a}$ and $f_{c, a}$. In equation (2), the similarity parameters $R e_{m}$ and $f_{m}$ are independent of the length scale used to reduce the data to nondimensional form. For rectangular or annular passages, and with $R e_{c, r}=2,100$ as the reference, $\psi_{R}$ decreases with increasing aspect or diameter ratio. For fully developed flow in smooth passages, the critical limiting (Fanning) friction factor is about 0.008 , provided the data are reduced using the hydraulic (or equivalent) diameter, $D_{e}\left(=A / P_{w}\right)$. For triangular passages, $\psi_{R}$ increases with increasing height-to-base ratio or with decreasing apex angle in the case of isosceles channels.

To shed further light on the application of the law of corresponding state to flow passages, data for rectangular, annular and triangular passages (a total of 500 points) that were presented as three separate figures in the aforementioned paper have been consolidated into a single plot (Fig. 1). The well known laminar and turbulent relations, as well as the circular tube data of Dodge and Metzner (1959), are also included in Fig. 1 for purposes of comparison. The number of data points for each cross-section is given in brackets in Fig. 1. Given the differences in the relative entrance length $\left(L_{e} / D_{e}\right)$, the relative roughness of the smooth duct, and the lack of geometrically similar test conditions, all of which may affect the critical Reynolds number, the results in Fig. 1 are satisfactorily consistent. As might be expected, the turbulent relation is a good 


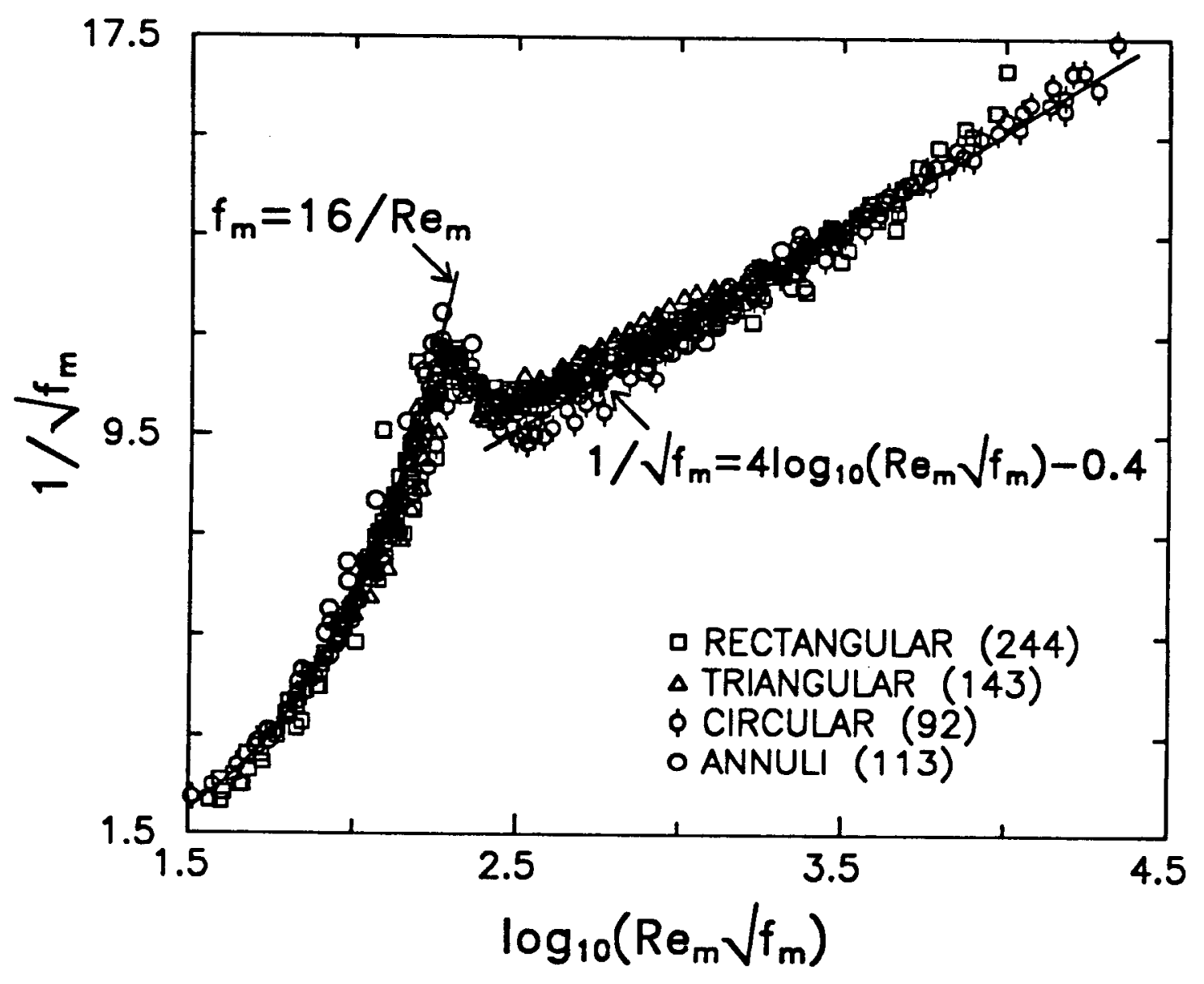

Figure 1. Friction factor for circular and noncircular passages. 
approximation of the data over a limited range. It was tempting to establish an improved relation for the turbulent results on this figure; however, it was decided that such an attempt must await the results of ongoing systematic experiments.

\subsection{Rough Passages.}

To date, efforts to provide a generalized approach for handling experimental data obtained on rough tubes or very clear insight into the mechanism of friction/heat transfer augmentation have been frustrated by several factors. In the first place, there are far too many variables (pitch, height, shape, angular orientation, density, etc.) associated with the problem. Second, as was the case until recently with smooth passages, the contentious issue has been the choice of the length scale needed to reduce the experimental data to nondimensional form. These are further complicated by the widely held view that the conventional Reynolds number is a similarity parameter, a position that is not necessarily correct. And, finally, although there are clear indications in the literature that the origin of turbulence must be the common feature for all types of roughness, it is rather surprising that this line of reasoning has not been explored theoretically or experimentally in the past. In fact, of the extensive studies on friction and heat transfer with enhanced tubes, only a few of these covered the complete range extending from the laminar through the turbulent regime, and nearly all of these were performed on circular tubes (Nunner, 1956; Koch, 1960).

It is most instructive to begin this analysis by considering the effects of roughness on the critical parameters for transition, and the relevant data are summarized in Table 1. To gain more insight into the influence of roughness on transition, the results of Koch (1960) are reproduced exactly in Fig. 2. For these results, the pitch-to-height ratio ( $p / e)$ was held fixed at 9.8 , hence the figure shows the generally expected trend with increasing height of roughness. It should be emphasized that the data of Table 1 as well as those 
Table 1: Summary of critical data for rough passages.

\begin{tabular}{|c|c|c|c|c|c|c|}
\hline Author(s) & $\overline{L_{\mathrm{e}} / D}$ & $f_{c, a} \times 10^{2}$ & $\overline{R e_{c, a}}$ & $\overline{p / e}$ & $e / D$ & Roughness Geometry \\
\hline \multirow{8}{*}{$\begin{array}{l}\text { Nunner (1956) } \\
\text { (fluid - air) }\end{array}$} & \multirow{8}{*}{50} & $0.83^{1}$ & 2510 & 7 & 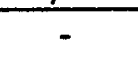 & (smooth tube) \\
\hline & & 1.31 & $1988^{2}$ & 20 & 0.04 & $\begin{array}{l}\text { transverse rectangular } \\
\text { ring inserts }\end{array}$ \\
\hline & & 1.31 & $1988^{2}$ & 20 & 0.04 & \multirow{6}{*}{$\begin{array}{l}\text { transverse semi-circular } \\
\text { ring inserts with no } \\
\text { clearance at the wall }\end{array}$} \\
\hline & & 0.95 & 2537 & 80 & 0.04 & \\
\hline & & 1.14 & 2497 & 2 & 0.08 & \\
\hline & & 1.65 & 2014 & 5 & 0.08 & \\
\hline & & 1.92 & 1653 & 10 & 0.08 & \\
\hline & & 3.36 & 1022 & 20 & 0.08 & \\
\hline \multirow{9}{*}{$\begin{array}{l}\text { Koch (1960) } \\
\text { (fluid - air) }\end{array}$} & \multirow{9}{*}{50} & 4.62 & 1019 & 3.92 & 0.1 & \multirow{6}{*}{$\begin{array}{l}\text { transverse disc-shaped } \\
\text { inserts with no clearance } \\
\text { at the wall }\end{array}$} \\
\hline & & 3.68 & 972 & 9.8 & 0.1 & \\
\hline & & 3.72 & 747 & 65 & 0.1 & \\
\hline & & 47.33 & 226 & 3.92 & 0.2 & \\
\hline & & 28.88 & 244 & 9.8 & 0.2 & \\
\hline & & 57.86 & 181 & 26.0 & 0.25 & \\
\hline & & 3.16 & 1170 & 9.8 & 0.045 & \multirow{2}{*}{$\begin{array}{l}\text { transverse ring inserts } \\
\text { with clearance for flow } \\
\text { near the wall }\end{array}$} \\
\hline & & 7.17 & 702 & 9.8 & 0.075 & \\
\hline & & 0.80 & $\begin{array}{c}2944 \\
(2900)^{3}\end{array}$ & - & - & (smooth tube) \\
\hline $\begin{array}{l}\text { Marner \& } \\
\text { Bergles (1978) } \\
\text { (fluid - ethylene } \\
\text { glycol) }\end{array}$ & - & $\overline{\overline{3.11}}$ & 1133 & $\overline{6^{4}}$ & $0.088^{4}$ & forge-fin tubes \\
\hline \multirow{2}{*}{$\begin{array}{l}\text { Molloy (1967) } \\
\text { (fluid - air) }\end{array}$} & $\overline{8.1}$ & $\overline{0.90}$ & $\overline{2203}$ & - & $=$ & \multirow{2}{*}{$\begin{array}{l}\text { (smooth) } \\
\text { spirally wound wire }\end{array}$} \\
\hline & 8.1 & 0.78 & 2659 & 10 & 0.014 & \\
\hline \multirow{7}{*}{$\begin{array}{l}\text { Knudsen \& } \\
\text { Katz }(1950)^{5} \\
\text { (fluid - water) }\end{array}$} & 30.9 & $\overline{1.3}$ & $\overline{1766}$ & - & - & (smooth) \\
\hline & 33.0 & 1.5 & 1502 & 0.952 & 0.089 & \multirow{6}{*}{$\begin{array}{l}\text { helically-finned } \\
\text { annular tubes }\end{array}$} \\
\hline & 42.1 & 3.0 & 784 & 1.15 & 0.184 & \\
\hline & 41.1 & 2.1 & 1093 & 0.513 & 0.185 & \\
\hline & 50.1 & 3.9 & 605 & 0.730 & 0.236 & \\
\hline & 52.9 & 3.5 & 666 & 0.452 & 0.250 & \\
\hline & 54.3 & 4.4 & 536 & 0.299 & 0.269 & \\
\hline \multirow{5}{*}{$\begin{array}{l}\text { Li et al. (1982) } \\
\text { (fluid - water) } \\
\text { (visual studies) }\end{array}$} & & - & 1700 . & 11.83 & 0.047 & \multirow{4}{*}{ helically-ridged tubes } \\
\hline & & - & 1700 & 29.6 & 0.048 & \\
\hline & 30 & - & 2200 & 67.7 & 0.058 & \\
\hline & & - & 2000 & 99.9 & 0.040 & \\
\hline & & - & 2300 & - & - & (smooth) \\
\hline
\end{tabular}




\begin{tabular}{lcccccl}
\hline \hline Author(s) & $L_{e} / D$ & $f_{c, a} \times 10^{2}$ & $R e_{c, a}$ & $p / e$ & $e / D$ & Roughness Geometry \\
\hline Watkinson & 29.9 & 3.10 & 938 & 10.0 & $0.097(1)^{6}$ & \\
et al. (1974) & 35.6 & 2.77 & 756 & 13.0 & $0.105(2)$ & \\
(fluid - oil) & 43.6 & 3.21 & 989 & 20.0 & $0.118(3)$ & \\
& 51.6 & 3.18 & 949 & 14.5 & $0.157(4)$ & High Spiral Fins (HSF) \\
& 57.1 & 2.83 & 693 & 33.3 & $0.144(5)$ & \\
& 24.0 & 3.44 & 886 & 6.0 & $0.088(9)$ & \\
& 20.1 & 11.35 & 731 & 6.6 & $0.149(18)$ & \\
& 33.8 & 1.65 & 1464 & - & $0.099(11)$ & \\
& 52.9 & 2.18 & 1299 & - & $0.148(13)$ & \\
& 42.9 & 2.63 & 1112 & - & $0.111(4)$ & High Straight Fins (STF) \\
& 52.5 & 1.97 & 1290 & - & $0.094(16)$ & \\
& 24.1 & 2.53 & 1483 & - & $0.073(20)$ & \\
& 23.8 & 2.37 & 2010 & 162.2 & $0.037(15)$ & \\
& 30.5 & 1.81 & 1296 & 206.9 & $0.037(17)$ & \\
& 19.4 & 3.71 & 2127 & 242.0 & $0.026(19)$ & Low Spiral Fins (LSF) \\
& 24.6 & 1.93 & 1482 & 164.6 & $0.042(21)$ & \\
& 41.5 & 1.49 & 1579 & 444.4 & $0.047(22)$ & \\
& 41.3 & 1.54 & 1478 & 181.8 & $0.057(23)$ & \\
\hline \hline
\end{tabular}

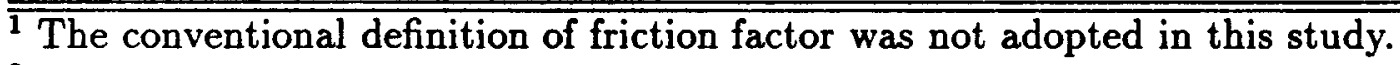

${ }^{2}$ Characterized by the same profile in the laminar and transition regions.

${ }^{3}$ Smooth tube critical value reported in original study.

4 Values were deduced from Watkinson et al. (1974) for Tube \# 9.

5 The characteristic dimension is the hydraulic diameter, $D_{\mathrm{c}}=d_{o}-d_{i}$.

${ }^{6}$ Numbers in brackets correspond to tube numbers in the original paper. 


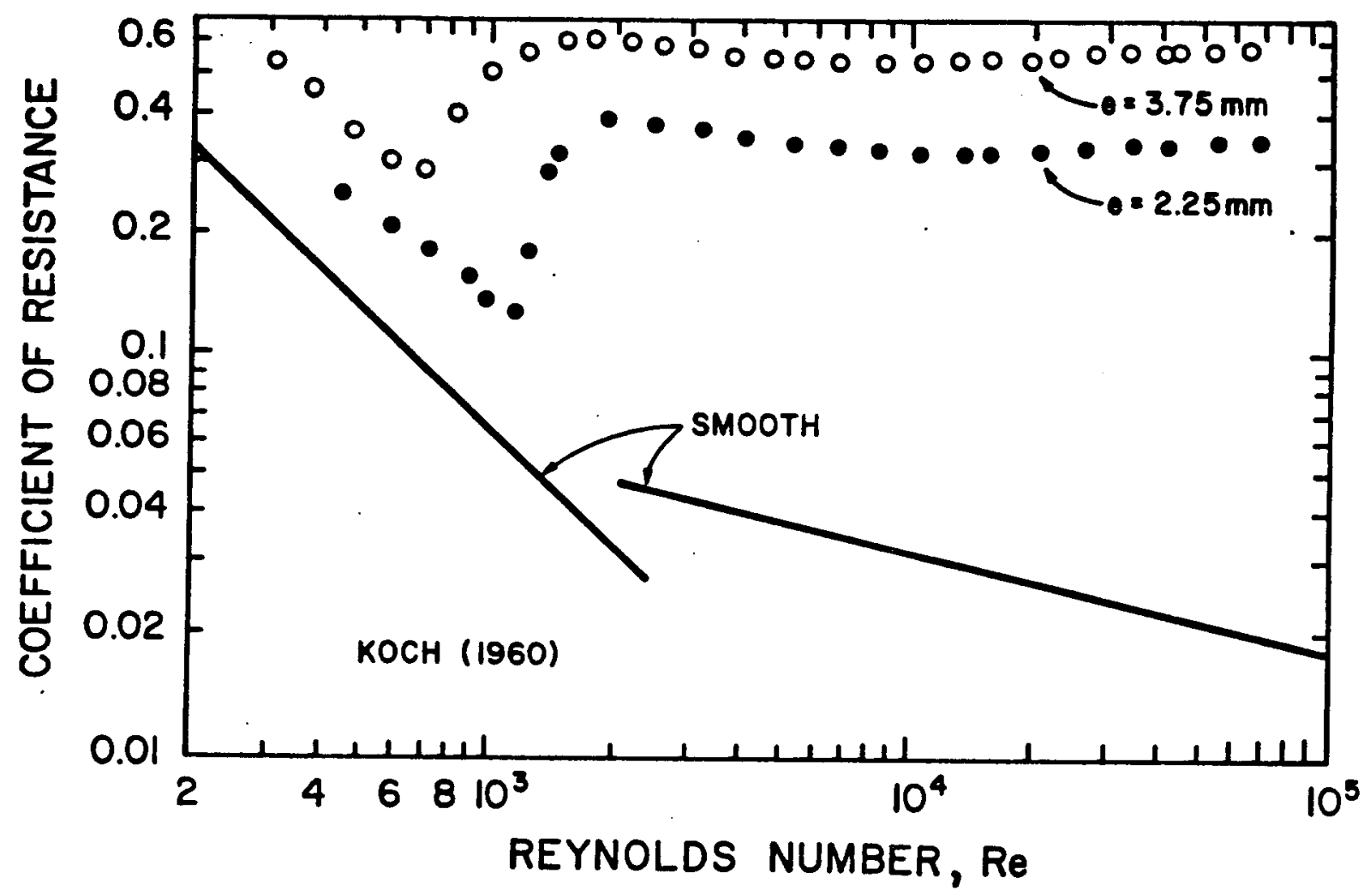

Figure 2. Effect of $e / D$ on resistance coefficient according to Koch (1960). 
on Fig. 2 were obtained in the absence of heat transfer (isothermal conditions). Also, to provide a consistent basis for viewing of the critical parameters, the characteristic length for the results in Table 1 is the maximum internal tube diameter. The only exception occurs with the results of Knudsen and Katz (1950) which, due to the use of annular passages, have the hydraulic diameter as the linear parameter.

From a comparison of the results (Table 1) of Nunner and Koch, both of which were obtained using the same experimental facility, it may be noted that Nunner's $f_{c, a}$ data are much lower, while the values for $R e_{c, a}$ are substantially higher, than those determined from Koch's results. These cannot be attributed solely to differences in the details of roughness. Nunner defined the resistance coefficient in terms of the difference between the conventional pressure drop and the work due to acceleration; the latter being expressed as a function of both the pressure and temperature at the inlet and exit of the test section. Obviously, even for isothermal conditions, with nearly the same temperature at the inlet and exit, the latter term always enters into the calculation since the inlet and exit pressures are unequal. Without data for pressure and temperature at the two end points for each trial, the usual friction factor cannot be established from his data. Also, the characteristic length in the Reynolds and Nusselt number was the mean diameter, $d_{m}$, defined as the equivalent diameter of a smooth pipe having the same volume per unit length. Using the $d_{m}$ values in his paper (c.f. Table 2), all of his results were re-computed using the internal diameter as reference. Even with this revision, the critical $R e$ values are still considerably higher than would be expected for the test conditions. The reader may wish to compare the values in Table 1 with those in Table 2 of the original paper. By contrast, in the subsequent study of Koch, the usual definition of friction factor was used and the length parameter was the tube diameter. Suffice it to state that using the empirical relation provided by Koch, it has been estimated that 
$R e_{c, a}=1700$ represents the upper limit to the range of critical Reynolds numbers for Nunner's test conditions. The results of other investigations (Table 1) definitely support this view.

A study of the results in Table 1 and Fig. 2 reveals a number of important trends. The most obvious of these is that, the critical friction factors are considerably higher, while the critical Reynolds numbers are substantially lower, than the values for smooth tubes. In other words, $\psi_{f}<1$ and $\psi_{R}>1$ when the data are reduced using the internal diameter. In this regard, it is worthy of note that, if the hydraulic diameter $D_{e}$ were used as the linear parameter, the critical friction factor can be lower than the smooth tube value, and the values for $R e_{c, a}$ would be even lower than those in Table 1 . At this point it is useful to recall that for smooth passages and with $D_{e}$ (i.e. $\psi_{f} \simeq 1$ ), $\psi_{R}<1$ for rectangular or annular tubes, but $\psi_{R}>1$ for triangular passages.

Another important trend is that $f_{c, a}$ increases, while $R e_{c, a}$ decreases, markedly with increasing height of roughness, regardless of whether one is dealing with transverse repeated-rib inserts, fins, or spirally ridged surfaces. From these results it appears that the roughness height is by far the single most important parameter, at least insofar as the influence on transition is concerned, with moderate effect of pitch-to-height ratio, $p / e$. Given the available data base, the fact that the effects of all relevant parameters cannot be established definitely is of little consequence to the present considerations. It is, however, correct to state that the global effect of roughness is to bring about early transition, regardless of the geometric configuration of roughness or the prevailing local flow conditions (i.e. separation and reattachment, swirl, etc.).

To shed further light on the influence of roughness on transition, the results in Table 1 are presented in several ways in Figs. 3-5. The first of these is a plot of the normalized critical Reynolds number, $\psi_{R}\left(=R e_{c, r} / R e_{c, a}\right)$, versus the relative height of 


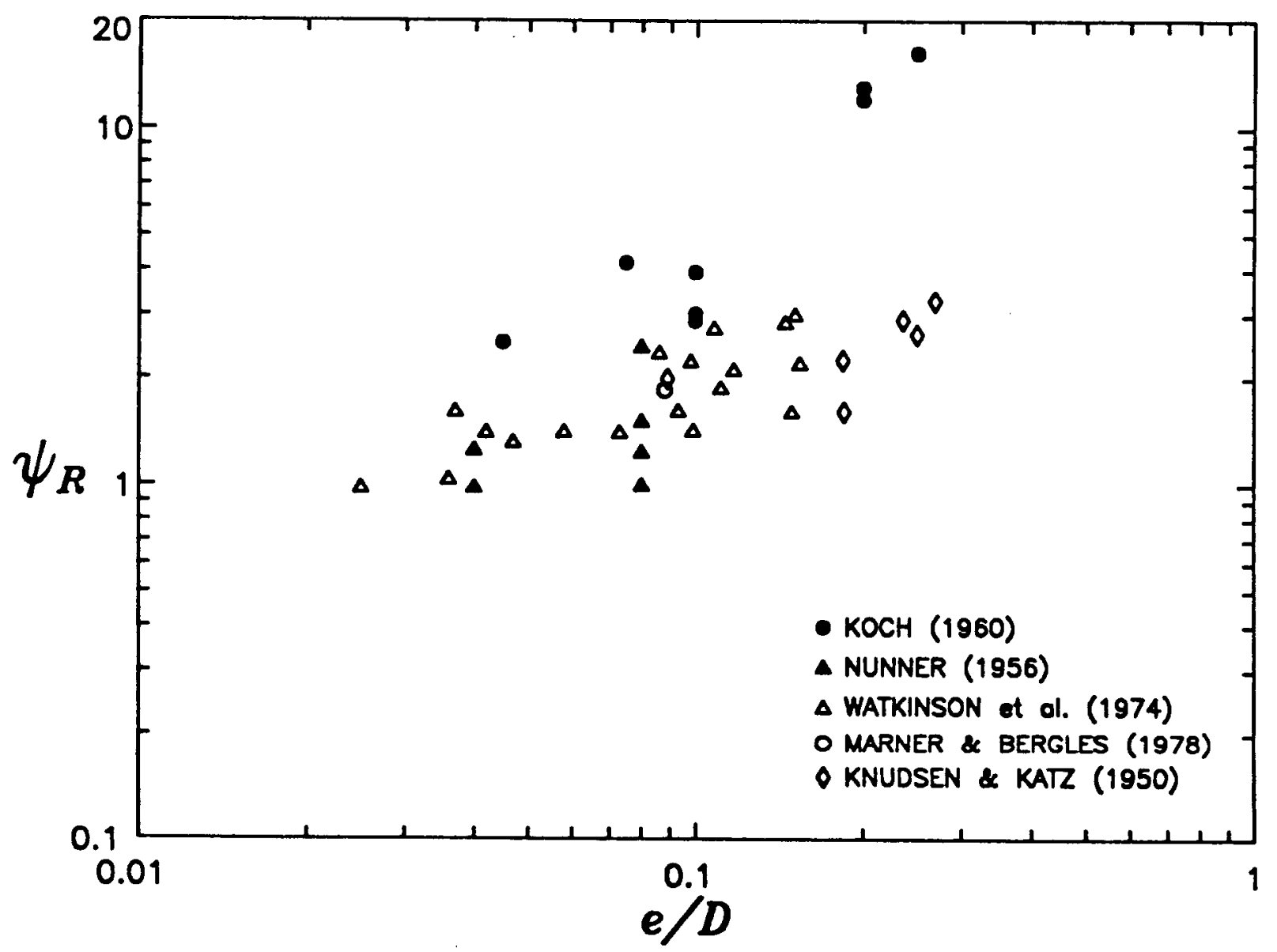

Figure 3. Variation of $\psi_{R}\left(=R e_{c,} / R e_{c, a}\right)$ with $e / D$. 


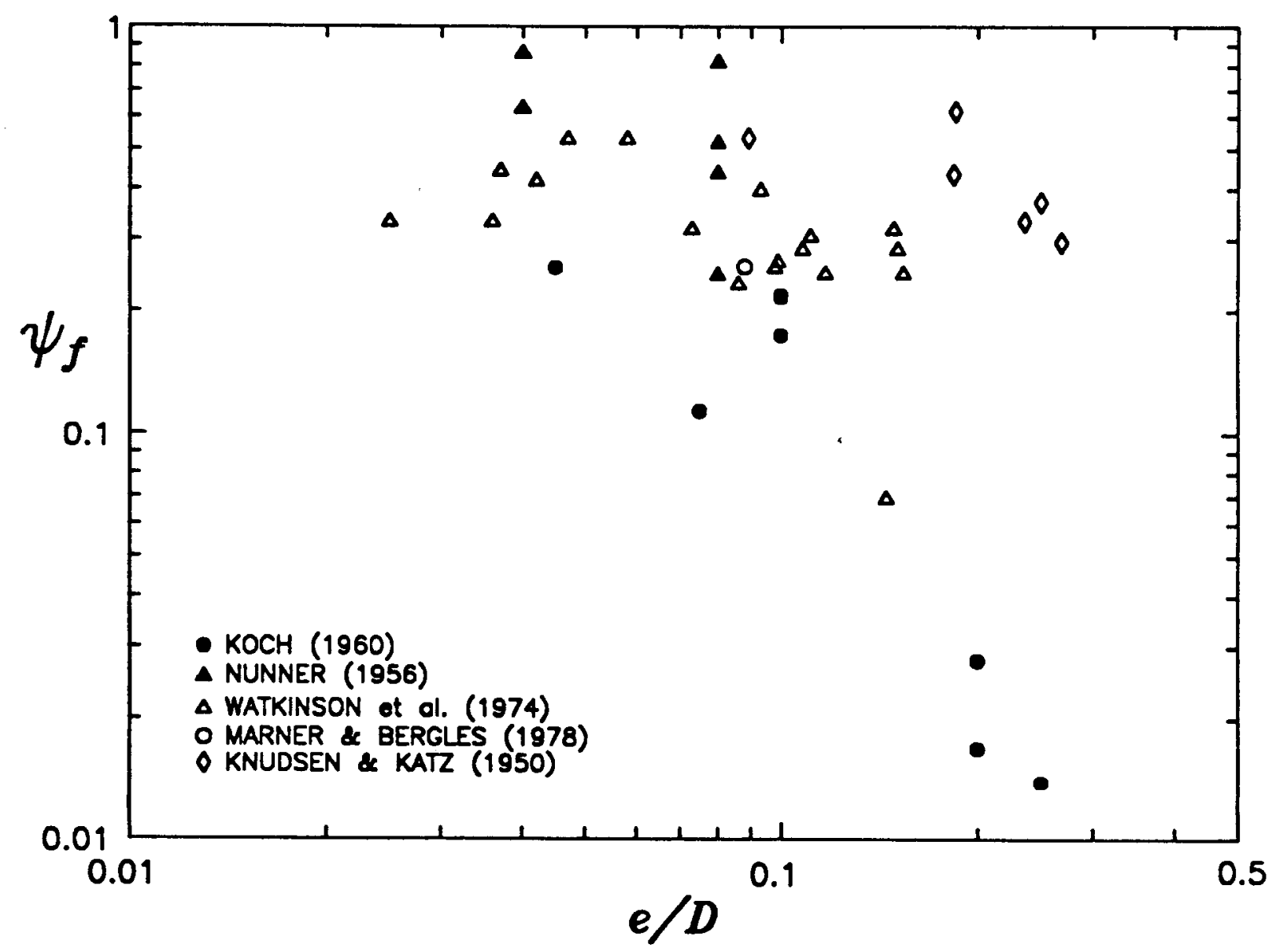

Figure 4. Variation of $\psi_{f}\left(=f_{c,} / f_{c a}\right)$ with $e / D$. 


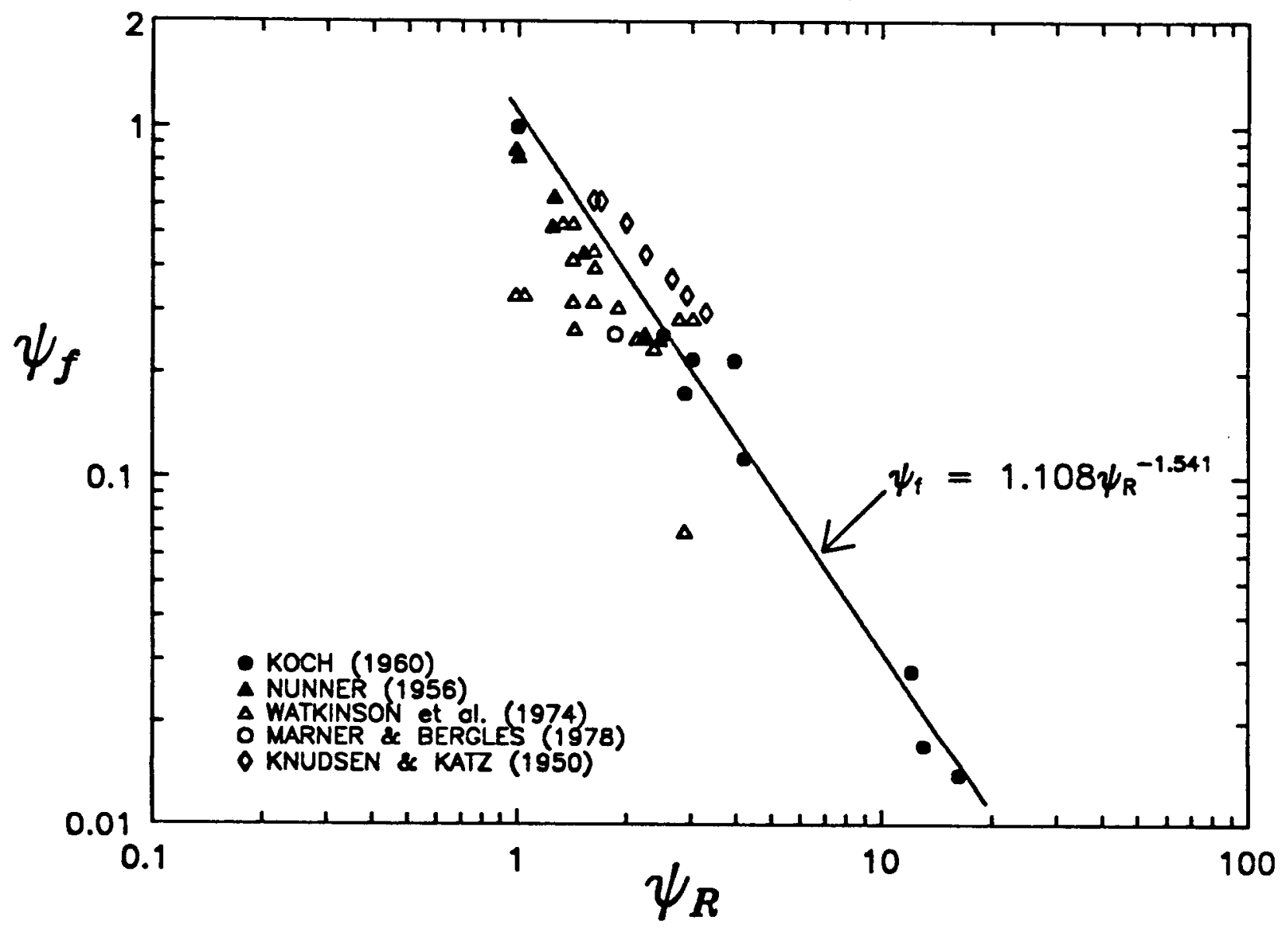

Figure 5. Variation of $\psi_{f}$ with $\psi_{R}$. 
roughness $(e / D)$ while the second shows the variation of $\psi_{f}\left(=f_{c, r} / f_{c, a}\right)$ with $e / D$. Figure 5 is a plot of $\psi_{f}$ versus $\psi_{R}$ for all $e / D$. The line in this figure represents a logarithmic least squares fit using the data of Koch. For the data of Nunner and Koch, the appropriate smooth tube values (Table 1) were used to compute $\psi_{f}$ and $\psi_{R}$, while the data of Marner and Bergles, and Watkinson et al. were scaled with the limiting smooth tube values (i.e. $f_{c, r}=0.008$ and $R e_{c, r}=2,100$ ). Although Watkinson et al. (1974) reported a critical Reynolds number of 1300 for smooth tubes, it was determined from the presentation therein that this value was most likely deduced from their heat transfer data for Tube \#10 with a relative entrance length of $L_{e} / D=24$. Since ten of their tubes were characterized by $L_{e} / D>30$ (Table 1), use of the circular tube values is justified, at least for those tubes.

Excluded from the analysis are the qualitative results of $\mathrm{Li}$ et al. and the results of Molloy. The critical Reynolds numbers of Li et al. were determined via flow visualization, hence no data for the critical friction factor are available. Given the difficulty in obtaining accurate pressure drop measurements in the laminar and transition regions, as noted by Molloy in his report, and the rather unusual trends with increasing Reynolds number, his data are unreliable and hence must be rejected. His claim that there is a range in the transition region where the rough tube could actually give lower friction factors than the smooth tube is neither correct when the tube diameter is the linear parameter nor consistent with his heat transfer results.

Fig. 3 shows that $\psi_{R}$ increases with $e / D$ and that, for a given $e / D$, disc inserts which are essentially repeated-rib roughness give values that are generally higher than for finned tubes. In this regard it is useful to recall that the lower the critical Reynolds number, the greater the critical parameter, $\psi_{R}$. Consistent with the effects of $e / D$ on $f_{c, a}$ (Fig. 2) and the general trend of Fig. 3, the behavior in Fig. 4 is one of decreasing 
$\psi_{f}$ with increasing $e / D$ based on the results of Koch, while no marked variation with $e / D$ can be established from the data for finned tubes. From this lack of strong effect of $e / D$ on $\psi_{f}$ or $\psi_{R}$, it is reasonable to speculate that a similar trend would result from an analysis of heat transfer data. The most useful information that can be inferred from the results of Fig. 5 is the possible existence of a unique relation between $\psi_{f}$ and $\psi_{R}$ for all types of roughness. Using the nine data points of Koch, i.e. inclusive of the smooth tube value $\left(\psi_{f}, \psi_{R}\right)=(1,1)$, the applicable relation is:

$$
\psi_{f}=1.108 \psi_{R}^{-1.541}
$$

The correlation coefficient for equation (3) is -0.988 . It is worthy of note that the remaining data tend to cluster around this regression line. It is a fact of striking significance that the results on each of Figs. 3-5 follow two distinct trends: one for all finned tubes and the other for transverse repeated-rib inserts. It is also quite evident that some of Nunner's data tend to lie above or below those for finned tubes, clearly supporting an earlier observation about his results. Since the critical parameters are central to a proper interpretation of the role of transition on heat transfer, we will return to Figs. 3-5 in Section 3.2 .

Having discussed the influence of roughness on the critical parameters for transition, it is necessary to consider the fundamental requirement for flow similarity which, as noted previously on several occasions, asserts that the critical parameters must be the same. On this basis, it is quite clear that the three flows of Fig. 2 are not similar, at least not for the $R e>700$ region, because the onset of transition occurs at $R e=702$, 1170 and 2944 for $e=3.75 \mathrm{~mm}, 2.25 \mathrm{~mm}$ and 0.0 (smooth), successively. This observation makes it perfectly clear that, for a given $R e$ based on $D_{e}$, the number obtained from the division of the roughened tube data by the corresponding smooth tube value is not truly representative of the overall influence of roughness. To obtain realistic informa- 
tion on the physical situation, it is necessary to reduce the data using equations (1) and (2). Accordingly, the original results of Koch (1960) and Nunner (1956), which are the most extensive, have been re-analyzed, and these are shown graphically in Figs. 6 and 7 respectively where, in each case, the reduced friction factor $f_{m}$ is plotted against the reduced Reynolds number $R e_{m}$. The fact that Nunner's definition of the resistance coefficient is different and the somewhat higher $R e_{c, a}$ values from that study, do not preclude this general treatment of his data. Since the friction factor is practically independent of Reynolds number in the fully rough regime, these discrepancies are not expected to have a marked effect on the qualitative interpretation of Nunner's results.

Figs. 6 and 7 show that, when reduced according to the law of corresponding states, $f_{m}$ in the laminar region is almost independent of the geometric details of the roughness elements and its profile with increasing $R e_{m}$ is approximated by the smooth tube relation. In the turbulent regime, it is especially noticeable that, relative to the smooth tube data, the absolute increases in $f_{m}$ are considerably smaller than can be inferred from the conventional $f$ vs. Re plots (Fig. 2). This fully demonstrates that the marked enhancement factors, as is widely documented in the literature, are intimately associated with transition. For example, whereas Fig. 2 gives enhancement factors of about 17.5 and 20 for $e=2.25$ and $3.75 \mathrm{~mm}$ and $R e=50,000$, the corresponding $f_{m} / f_{m, s}$ $\left(=\phi_{f}\right)$ values from Fig. 6 are about 5.8 and 4.5, respectively. In fact, on Figs. 6 and 7, the largest value for $\phi_{f}$ is no more than 10. And, further, it is quite evident that $f_{m}$ depends solely on the geometric parameters of the roughness.

It should be noted that the $p / e=9.8$ results for $e / D=0.045$ and 0.075 (Fig. 6) are for the situation where a flow path $(2.7 \mathrm{~mm}$ in width) was provided between the external circumference of the ring insert and the tube wall, while for $p / e=9.8$ and $e / D=0.1$, the disc-shaped inserts were rigidly supported to the inner surface of the tube with no 


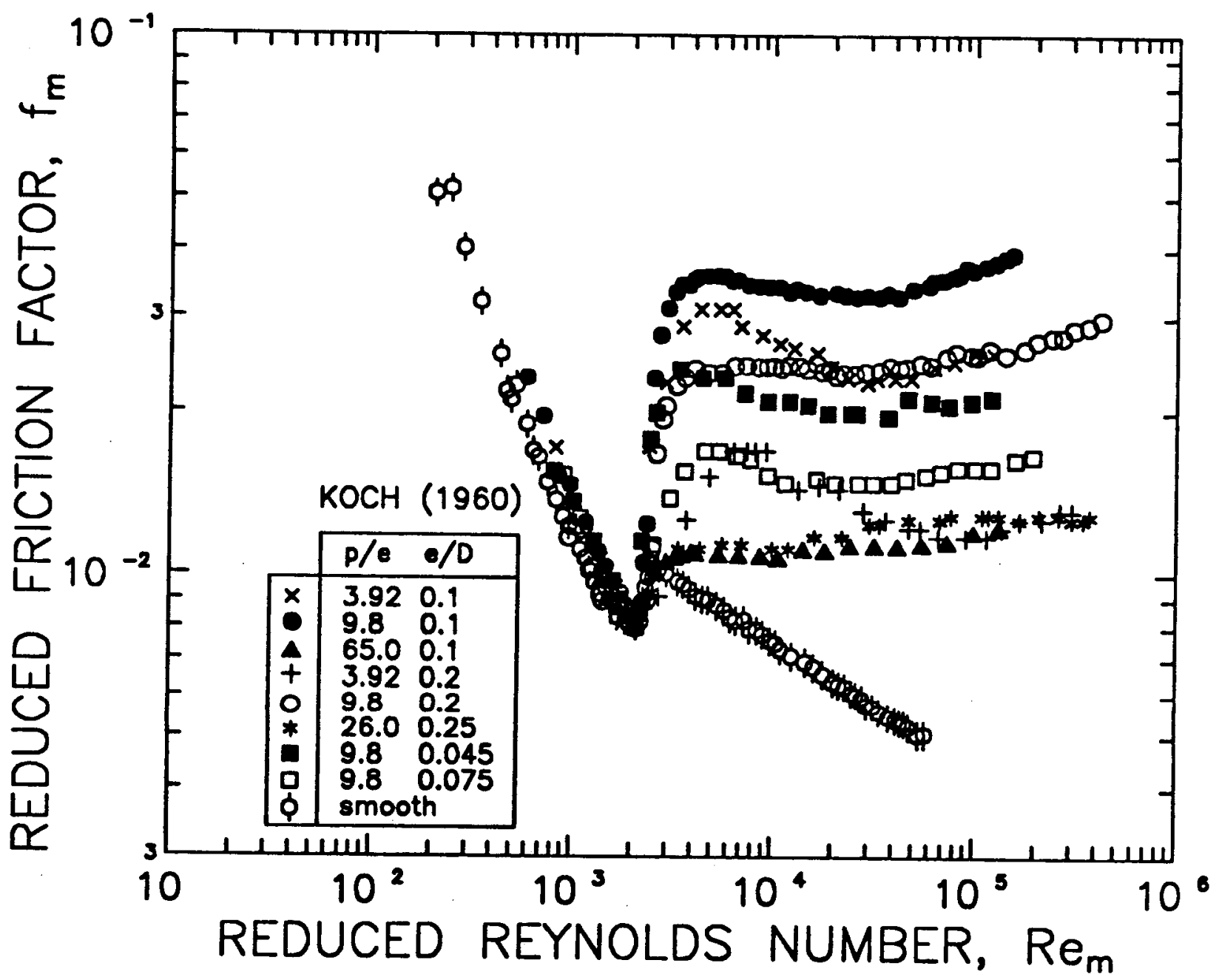

Figure 6. Similarity plot of $f_{m}$ vs. $R e_{m}$ using Koch's (1960) data. 


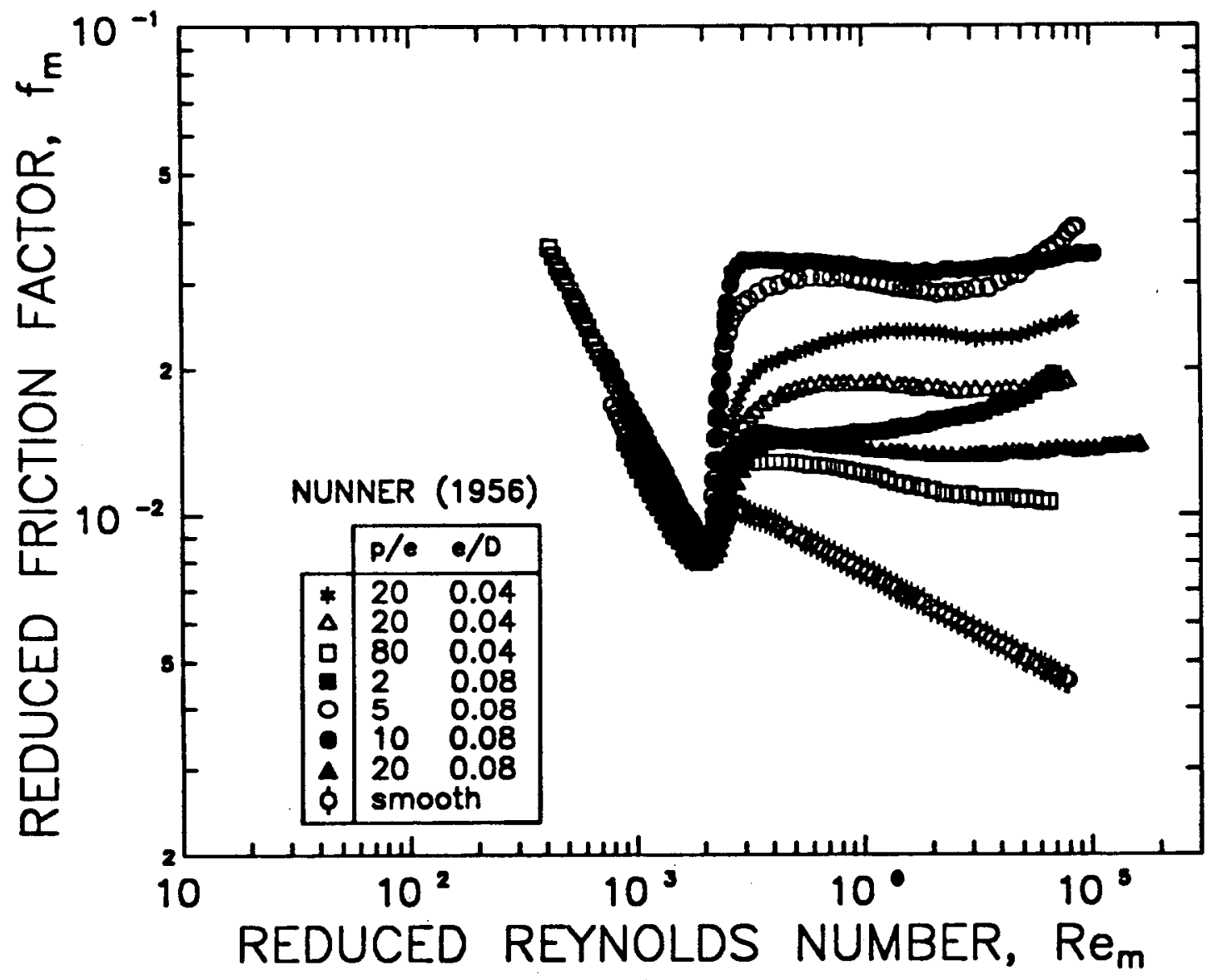

Figure 7. Plot of $f_{m}$ vs. $R e_{m}$ using Nunner's (1956) data. 
clearance. Also, in the case of Nunner's results (Fig. 7), the shape of the roughness elements and the number of such elements in a tube were additional parameters. For instance, with $e / D=0.08$, the number of rings for $p / e=10$ was double that for $p / e=$ 20 , while that for $p / e=20$ was 4 times that for $p / e=80$ with $e / D=0.04$. For these reasons, it would be unwise to draw any definite conclusion concerning the trend with increasing $e / D$ or $p / e$ from Figs. 6 and 7 .

From Fig. 6 or 7 the striking evidence is that the onset of the so-called fully rough regime is almost definite in character and in value, beginning right after the end of the transition region. In this regard the general features of these curves are remarkably similar to those presented by Nikuradse (1933) for the largest heights of sand-grain roughness (Fig. 8). Infact, even for the relatively small heights of roughness of that study, where the various curves progressively leave the smooth profile and where the points of successive departure vary with roughness height, it appears that the fully rough regime begins almost at the points at which the individual curves leave the smooth profile. ${ }^{1}$ Although many of the curves exhibit maxima and minima, in line with some of the profiles reported by Nikuradse, the depressions are such that, in each case, the error around the mean value is small. The only exception to this consistent trend can be seen in Fig. 7 for $p / e=3.92$ with $e / D=0.1$ and 0.2 . These curves are characterized by more pronounced maxima and minima. No definite statement can be made at this point about these trends.

The assertion that different types of roughness exhibit the same general features is strikingly illustrated in Fig. 8, which is a comparison of the reduced results of Fig. 2 with the data of Nikuradse (1933). Given the structural differences between sand-grain roughness and repeated-rib inserts as well as the attendant effects on transition, it would

\footnotetext{
${ }^{1}$ See, for example, H. Schlichting, Boundary Layer Theory, 7th ed., McGraw-Hill Book Company, N.Y., 1979, p. 617.
} 


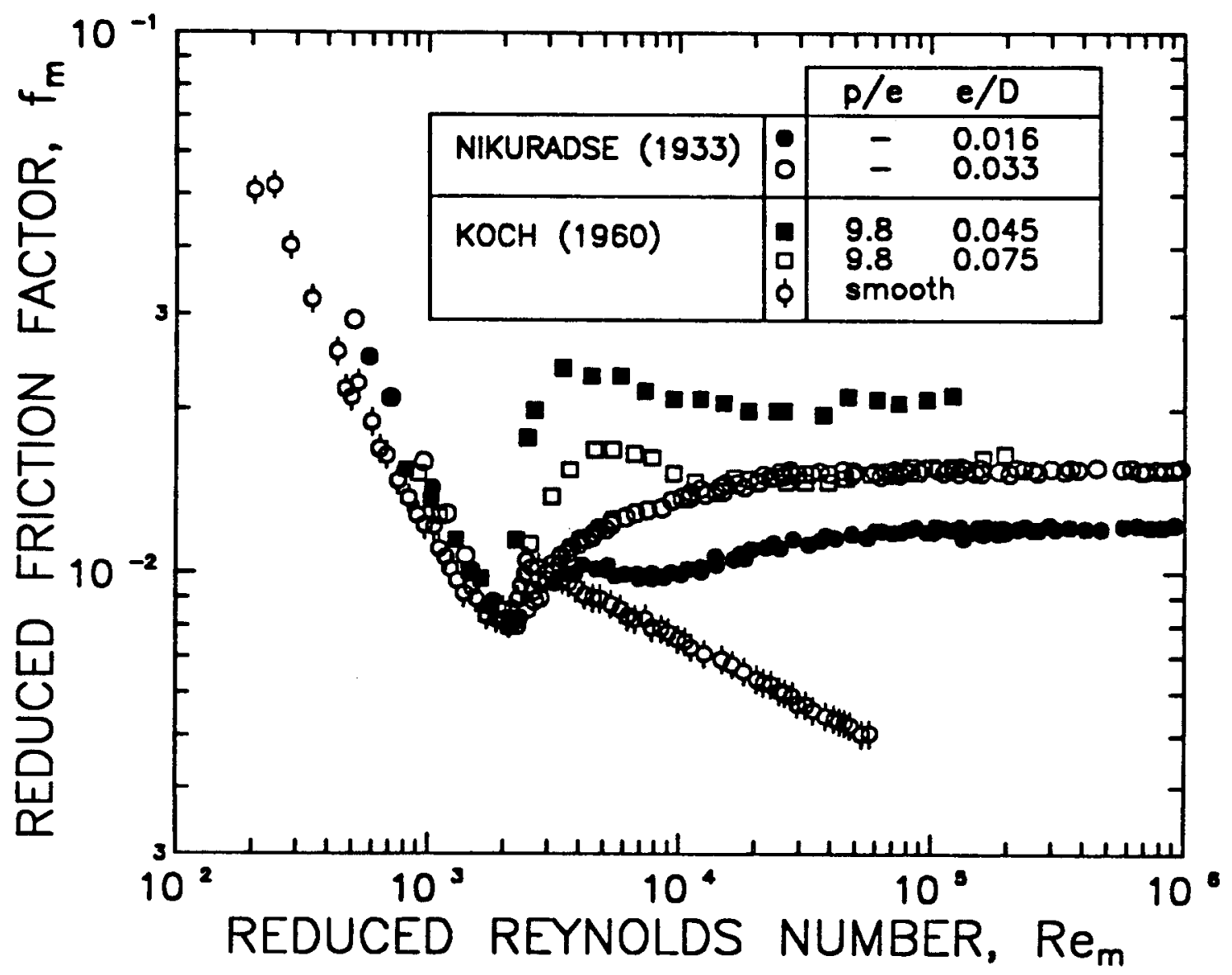

Figure 8. Nikuradse's sand-grain roughness vs. Koch's ring inserts. 
be unwise to draw definite conclusions concerning the modest agreement that is evident from this figure in the turbulent regime. Nevertheless, the comparison is important in two respects. First, it provides clearer insight as well as possible explanation as to why it has been possible to correlate experimental data using the friction similarity concept advanced by Nikuradse. Second, it definitely confirms an observation that the law of resistance in the fully rough regime should be of a universal nature for all pipes (Obot, 1988). Here, the relevant feature is the dependence of $f_{m}$ only on the geometric details of roughness, a trend that must also be expected for noncircular passages (Knudsen and Katz, 1950).

In summary, according to the law of corresponding states the reduced frictional pressure coefficients are essentially independent in laminar flow of, but are dependent in turbulent flow only on, the geometric parameters of roughness. In addition to establishing that the marked increases in friction factor are intimately associated with transition, the rather unique nature of the so-called fully rough regime has been isolated from similarity plots of $f_{m}$ vs. $R e_{m}$.

\section{ANALYSES OF HEAT TRANSFER IN FLOW PASSAGES}

Prior to the presentation and discussion of the heat transfer data, there are two important comments. First, for each of the studies used in the following analysis, the bases for the comparisons and conclusions are the smooth duct data for the particular study. Since the original smooth tube data for some of the studies were not approximated by the Dittus-Boelter or Petukhov-Popov correlation, it can be readily appreciated that the reduced data may not follow either correlation. Second, in the absence of the appropriate temperatures for these studies, no attempt was made to incorporate the Prandtl

number effect. As with the treatment of friction, this evaluation of the heat transfer 
results is divided into two main sections, one dealing with smooth and the other with enhanced passages.

\subsection{Smooth Passages.}

The role of transition in determining heat transfer in passages is best illustrated using the modified empirical Filonenko correlation, equation (4), and the Petukhov and Popov (1963) correlation, equation (5)

$$
\begin{gathered}
f_{m}=\left(1.58 \ln \psi_{R} R e_{a}-3.28\right)^{-2} \\
N u_{m}=\left(f_{m} \psi_{R} \operatorname{Re}_{a} \operatorname{Pr} / 2\right)\left[1.07+12.7\left(f_{m} / 2\right)^{1 / 2}\left(\operatorname{Pr}^{2 / 3}-1\right)\right]^{-1.0}
\end{gathered}
$$

Equations (4) and (5) are valid for circular and noncircular passages and, in the case of friction, the alternative form of equation (4) is verified in Fig. 1. It must be noted as a matter of particular importance that, by introduction of $f_{m}$ and $R e_{m}=\psi_{R} R e_{a}$ into the well known Petukhov-Popov correlation, the fundamental flow similarity requirement for all passages is satisfied.

Clearly, for a given $R e$ based on $D_{e}$ and quite consistent with the general effect of aspect ratio $\left(a_{r}\right)$ on friction factor (Obot, 1988), it is especially noticeable that $N u_{m}$ decreases with increasing aspect ratio. This is consistent with the results reported by several investigators (Drexel and McAdams, 1944; Washington and Marks, 1937). Likewise, for tubes of annular section which are characterized by $\psi_{R}<1$, the $N u_{m}$ trend with increasing diameter ratio $(\beta)$ is the same as noted above for rectangular channels, and this is definitely confirmed by the results of several researchers (Petukhov and Roizen, 1963; Koch and Feind, 1958). It is also quite apparent that as $\beta$ and $a_{r}$ approach zero and unity, respectively, the $N u_{m}$ values approach those for circular tubes, as might have been inferred from the general trend for $\psi_{R}$ with increasing $\beta$ or $a_{r}$ (Obot, 1988). 
Koch and Feind reported data for heat transfer and frictional pressure coefficients for $0.212 \leq \beta \leq 0.838$. The results for the latter were thoroughly discussed in Obot (1988). For heat transfer coefficients, laminar and turbulent data exhibit marked dependence on $\beta$, decreasing as $\beta$ is increased. Allowing for the fact that the critical Reynolds number increases with diameter ratio, their results have been re-analyzed and the $N u_{m}$ vs. $R e_{m}$ plots are given in Figs. 9 and 10. To obtain the corrections for the data of Fig. 9, the critical Reynolds numbers given in the paper cited above (c.f. Table 4) were used along with the rounded value of 2,100 for a circular tube. For Fig. 10, $R e_{c, r}=2719$ and this was extracted from the original paper.

For Figs. 9 and 10, although the results with and without the central core are almost indistinguishable, it may be noted that the data of Fig. 9 are about $25 \%$ higher than, while those for the latter figure are closely approximated by, equation (5). Fig. 10 indicates that, to calculate heat transfer coefficients for ducts of arbitrary shapes, equation (5) can give satisfactory results provided the appropriate critical parameters $\left(\psi_{f}, \psi_{R}\right)$ are used to account for the role of transition. The closeness with which the data for all $\beta$ are approximated by a single regression line also supports the view that heat transfer coefficients decrease with increasing $R e_{c, a}$. The trend on Fig. 9 also establishes the importance of determining the $R e_{c, r}$ value for any particular duct geometry.

For triangular passages, $\psi_{R}>1$ and $R e_{m}$ increases with increasing $h / b$ (or with decreasing apex angle in the case of isosceles ducts) for a given Reynolds number based on $D_{e}$. Consequently, $N u_{m}$ values can be substantially greater than for circular, rectangular or annular geometry with the same Reynolds number. For instance, using the $\alpha$ (apex angle) $=38.8^{\circ}$ data of Carlson (1959) which gives $\psi_{R}=1.197$ and for $R e=80,000$ (i.e. $R e_{m}=95,760$ ), the calculated $N u_{m}$ is about 163 , which is about $15 \%$ higher than with a circular tube. Infact, for the same $R e$, the calculated value for the narrowest, $\alpha=$ 


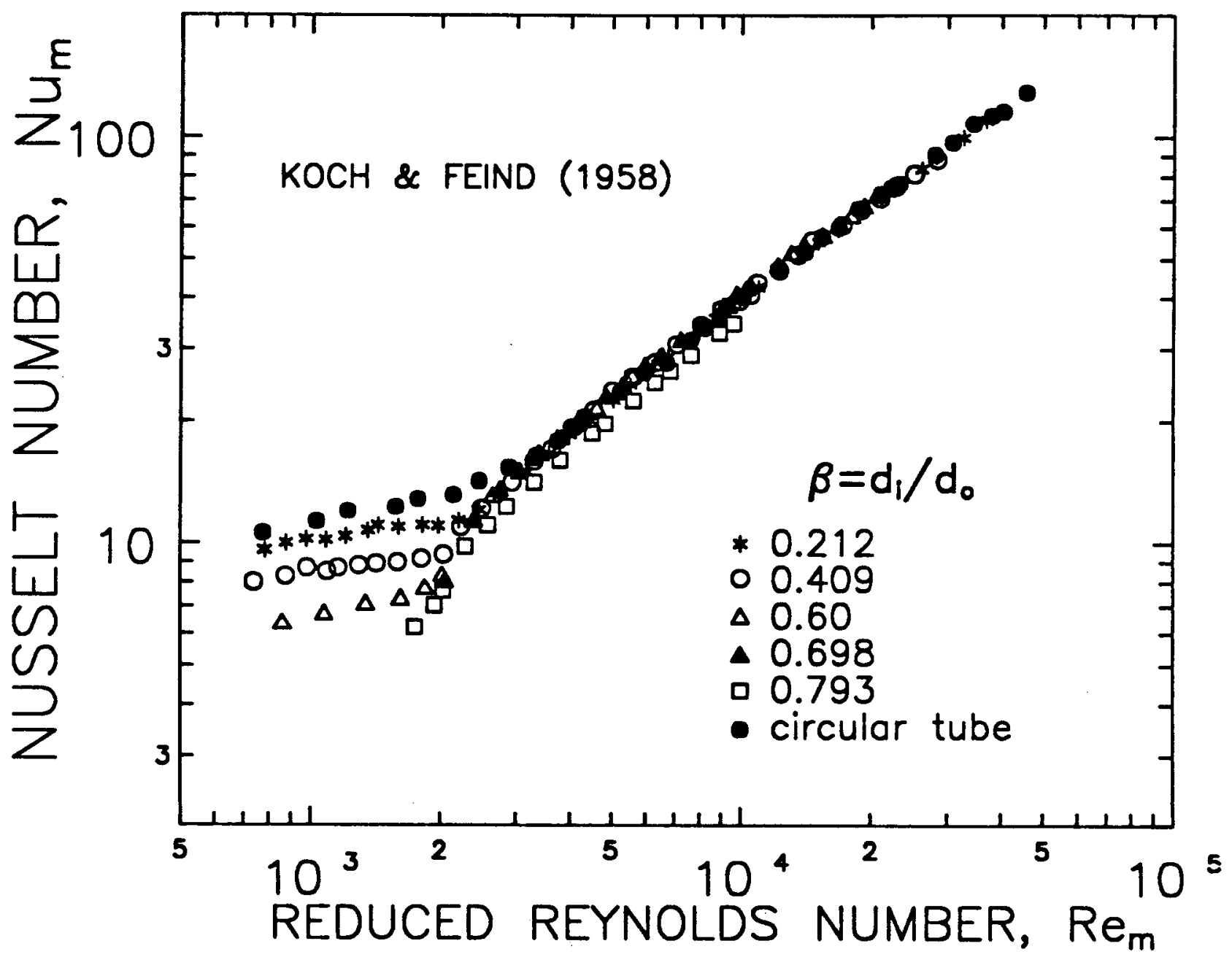

Figure 9. Nusselt number $\left(N u_{m}\right)$ vs. $R e_{m}$ for smooth annuli. 


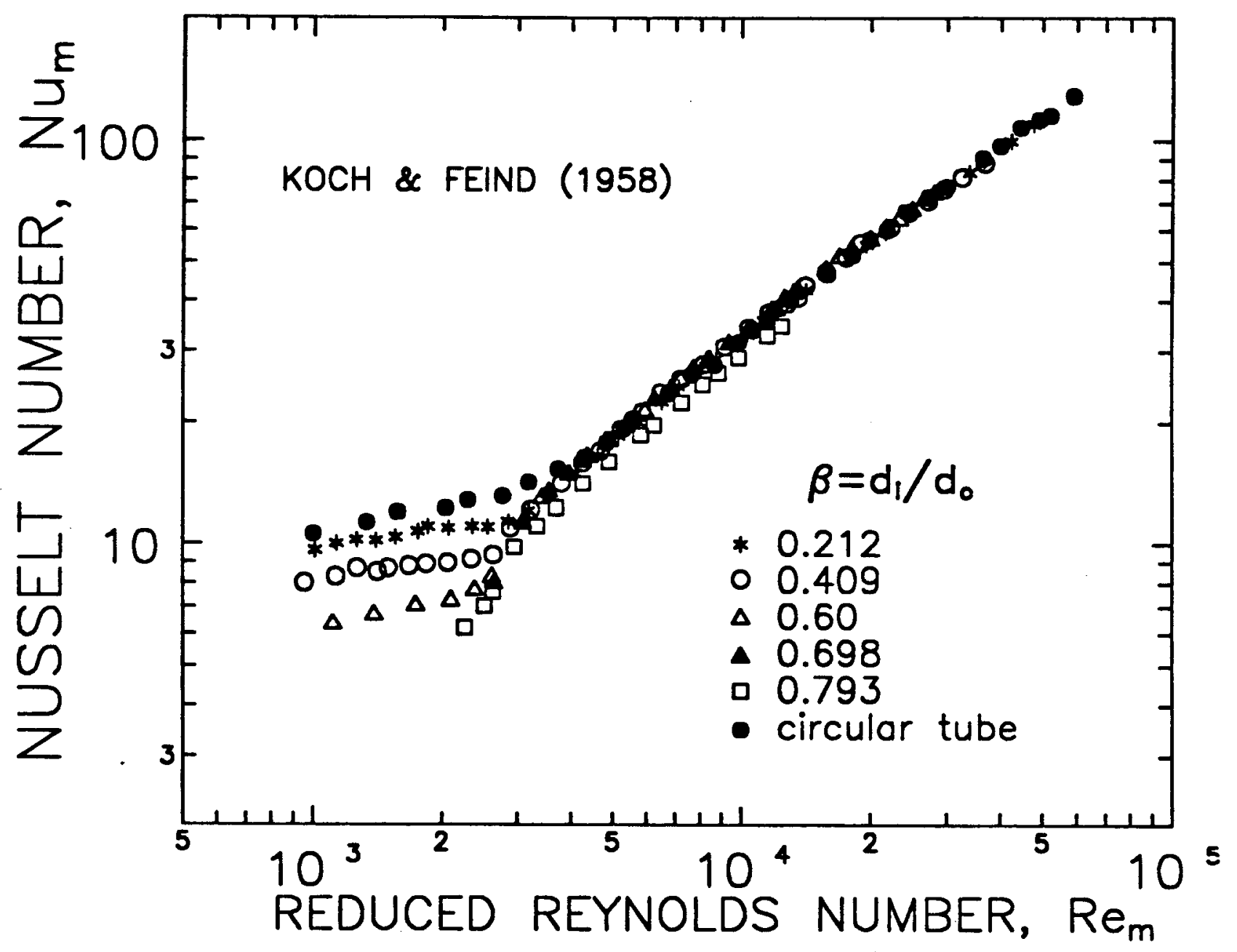

Figure 10. Nusselt number $\left(N u_{m}\right)$ vs. $R e_{m}$ for smooth annuli. 
$4.01^{\circ}$ passage $\left(N u_{m}=196\right)$ is about $40 \%$ and $60 \%$ higher than for a cylindrical and rectangular $\left(a_{r}=10\right)$ geometry, respectively. The common basis for these calculations is $\operatorname{Pr}=1$. This observation of great practical importance, though clearly at variance with the heat transfer results of Eckert and Irvine (1960) or Tokarev (1979), is not altogether surprising for several reasons. In the first place, to maintain a fixed Reynolds number (based on $D_{e}$ ) the average flow velocity must rise with increasing $h / b$, due to the reduction in flow area. And, further, the critical Re attains its lowest value for these narrow passages (Obot, 1988) and, even at such relatively low $R e$, the coexistence of laminar and turbulent flow (Eckert and Irvine, 1956; Bandopadhayay and Hinwood, 1973) should cause a marked improvement in heat transfer over that for completely laminar flow.

In summary, details such as those provided in this section, though totally obscured by the straightfoward application of the Reynolds number similarity as well as insuffcient understanding of the role of transition, are of considerable importance in several respects. First, they establish that the attainable friction factors and turbulent heat transfer coefficients in passages are determined largely by transition. For heat transfer the simple rule amounts to this: the lower the critical Reynolds number for transition, the greater the turbulent heat transfer coefficients. This provides the first concrete evidence that a comparative evaluation of the thermal performance of surfaces can be made simply from knowledge of the critical Re for transition. And, second, there are clear indications that, for a given Reynolds number based on $D_{e}$, heat transfer coefficients may be greatest in triangular passages, lowest for ducts of annular or rectangular geometry, with intermediate values for circular tubes. It remains, however, to generalize the role of transition by considering other flow situations and this will be considered in the 
following sections.

\subsection{Rough Passages.}

Having discussed the general treatment of friction in rough passages, attention is now directed to heat transfer enhancement, a problem that has been studied quite extensively for well over half a century. Reviews of some aspects of the widespread literature have been carried out by several researchers (Rabas, 1989; Webb, 1987; Ravigururajan and Bergles, 1986; Dalle Donne and Meyer, 1977). The general view is that varying degrees of improvement can be realized with roughness. For instance, for tests with air, the improvements in heat transfer are rarely more than a factor of five (Koch, 1960). It was established in the preceding section that there exists a definite connection between transition and heat transfer in smooth passages. On the basis of such a complete treatment, it is reasonable to question whether a similar link exists for rough passages. The answer to such a question is the subject of this section.

As with enhanced tube friction, we begin the discussion by considering Koch's complementary heat transfer data for the friction results of Fig. 2, and these are reproduced on Fig. 11. From this figure, the most obvious conclusion is that $N u_{r}$ is not significantly affected by the variation in $e / D$. For $R e>10,000$, the enhancement factors, $\phi_{h}\left(=N u_{r} / N u_{s}\right)$ are roughly constant for a given $e / D$, and can be stated as 1.98 and 2.23 for $e=2.25$ and $3.75 \mathrm{~mm}$ respectively. Since these ratios are much lower than the corresponding friction factor ratios of Fig. 2, and since no meaningful conclusions can be reached from such comparisons, these $\phi_{h}$ values must be viewed in light of the results on Fig. 6. In this connection it is of some interest to note that, in each case, $\phi_{h}$ is no more than one-half the friction factor ratio, $\phi_{f}\left(=f_{m} / f_{m, s} ; f_{m, s}=\right.$ smooth tube value). We will elaborate on this later. Although there are no large differences between the heat transfer results for the two roughness heights, the consistent trend in the turbulent 


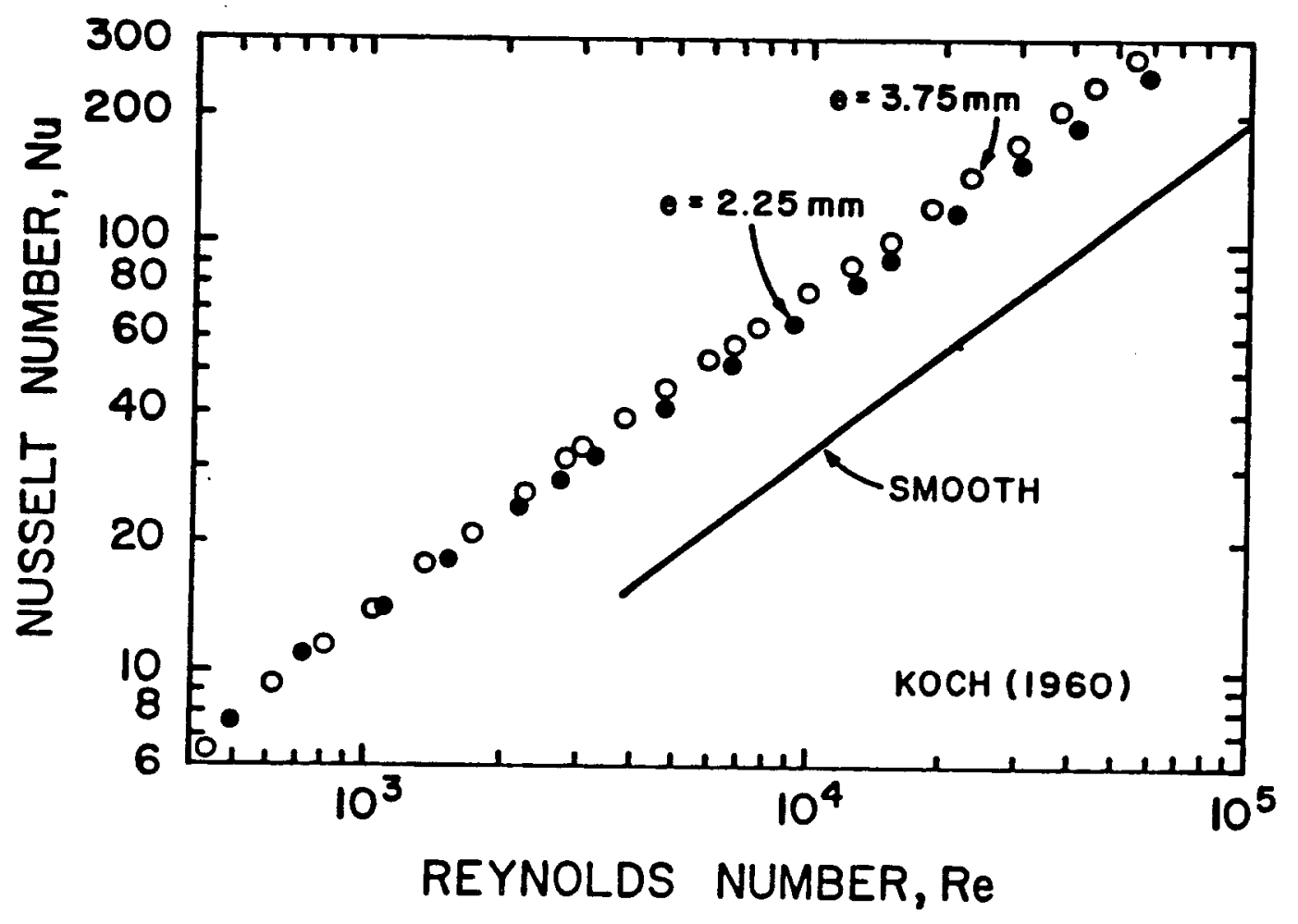

Figure 11. Effect of $e / D$ on Nusselt number according to Koch (1960). 
region is that the results for $e=3.75 \mathrm{~mm}$ are higher than for $e=2.25 \mathrm{~mm}$, in line with the depression of the onset of transition.

In order to gain clearer insight into the influence of transition on heat transfer, and in line with the presentation of Fig. 9 or 10, some of the results of Koch for which the critical Reynolds numbers were determined precisely have been re-analyzed and these are shown on Fig. 12 where $N u_{m}$ is plotted against the reduced Reynolds number, $R e_{m}$. It should be emphasized that as with Fig. 9 or 10, the correction has been applied only to the familiar Reynolds number. The $e=2.25$ and $3.75 \mathrm{~mm}$ results of Fig. 11 correspond to those for $e / D=0.045$ and 0.075 , respectively, and these are almost coincident with the smooth tube data. For the moment, the fact that some of the reduced data lie moderately above and below the smooth tube data does not matter, since the transition parameter $\left(\psi_{R}\right)$ is based on isothermal friction data. Although the fact that $\psi_{R}$ varies according to whether the fluid is being heated or cooled will be considered in Section 4, it should be emphasized that whatever might be the effects of heat transfer on $\psi_{R}$, these must be more pronounced for rough than smooth passages.

The general trends on Fig. 12 imply several things, virtually all of which are not easily predicted from much that has been written on the subject of convective heat transfer. That the reduced data with roughness cluster around the smooth tube data, with an average error of under $20 \%$, fully demonstrates that the marked increases in heat transfer coefficient are intimately associated with transition to turbulence. Second, it suggests that heat transfer coefficients can be predicted from the smooth tube relation provided the critical Reynolds numbers are known precisely. Third, from the critical Re data of Table 1 and Fig. 12 it is easily established that the fundamental law, which asserts that the lower the critical Reynolds number, the higher the heat transfer coeffcient, is satisfied for all cases. Finally, the law of corresponding state dictates that, in the 


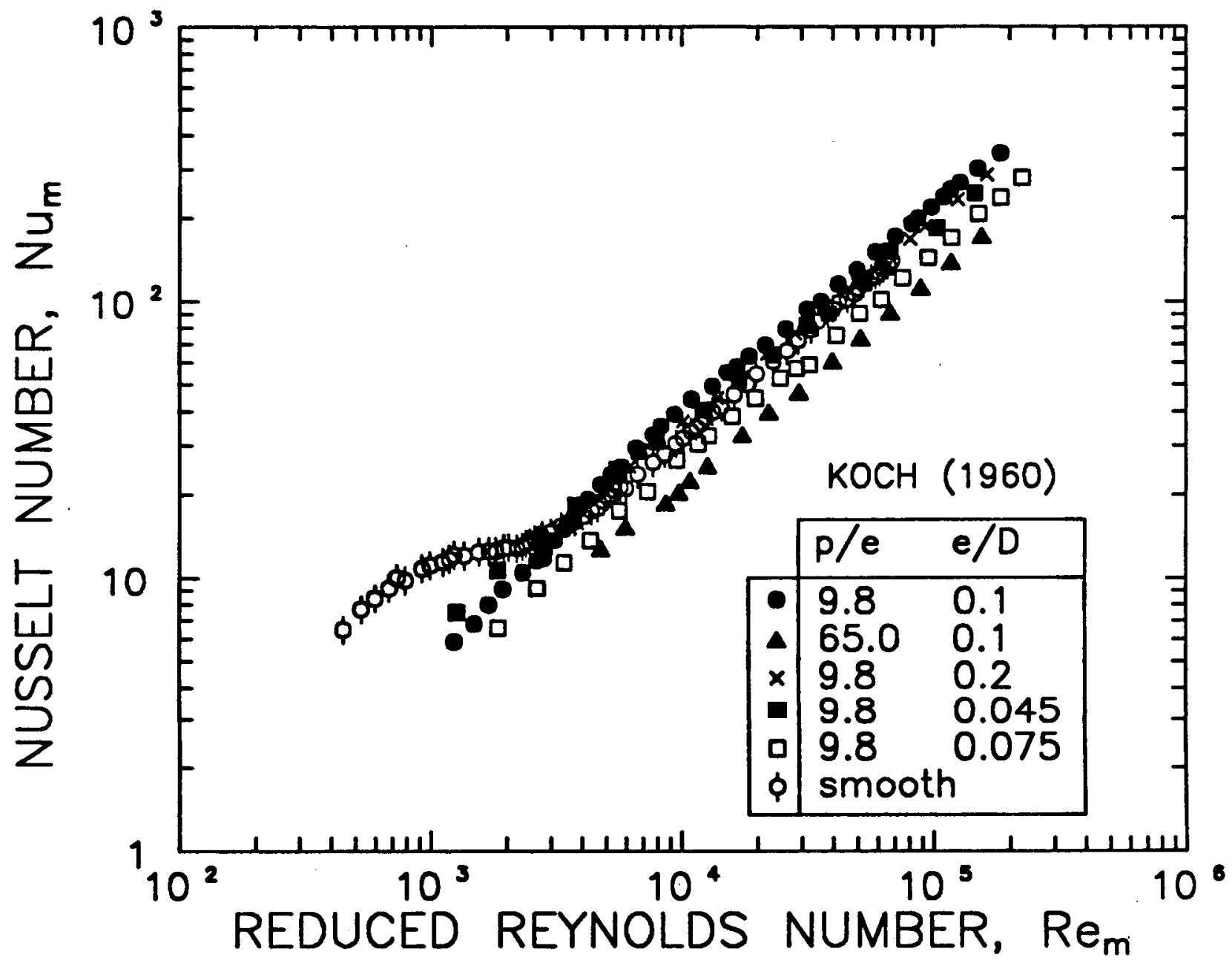

Figure 12. Nusselt number $\left(N u_{m}\right)$ vs. $R e_{m}$ using Koch's (1960) data. 
turbulent regime, there should be almost no departure from the conventional Reynolds number dependence for smooth tubes, and this has been carefully documented by numerous researchers (Knudsen and Katz, 1950; Koch, 1960; Gomelauri, 1964; Savage and Myers, 1963).

Figs. 13 and 14 which are alternative representations of the results of Nunner (1956) and Blackwelder and Kreith (1970) complement one another in that, unlike the use of $\psi_{R}$ values deduced from isothermal friction factor data, the $\psi_{R}$ values for these results were determined from the heat transfer data. In principle, this would be one of the preferred methods for determining $\psi_{R}$ because it eliminates its dependence on heat transfer (heating or cooling) and temperature. The difficulty, however, is that when $R e_{c, r}$ is unusually low, as was the case with some of Koch's test conditions, it becomes almost impracticable to obtain accurate heat transfer coefficients at such low flow rates. Whereas Nunner used repeated-rib inserts (Table 1), Blackwelder and Kreith tested twisted tape inserts and the relative lengths $\left(p_{t} / D\right)$, where $p_{t}$ is the length required for a $360^{\circ}$ rotation of the tape, are given on Fig. 14. In each case, it may be noted that virtually all of the reduced data lie in a band about $\pm 15 \%$ around the smooth tube data, fully supporting the view that good estimates of average heat transfer coefficients can be made provided the critical $R e$ for transition is known.

Another study uncovered during the exhaustive literature search, that by Knudsen and Katz (1950), dealt with heat transfer and pressure drop in annuli with transverse fins. The significance of this study relates to the use of water as the working fluid, in contradistinction to the air studies of Figs. 12-14. Another distinction between the air and water studies relates to the use of annular passages instead of circular tubes, inasmuch as the analysis should provide at least partial verification of the law of corresponding states for rough passages of complex shapes. In this connection it may be noted that 


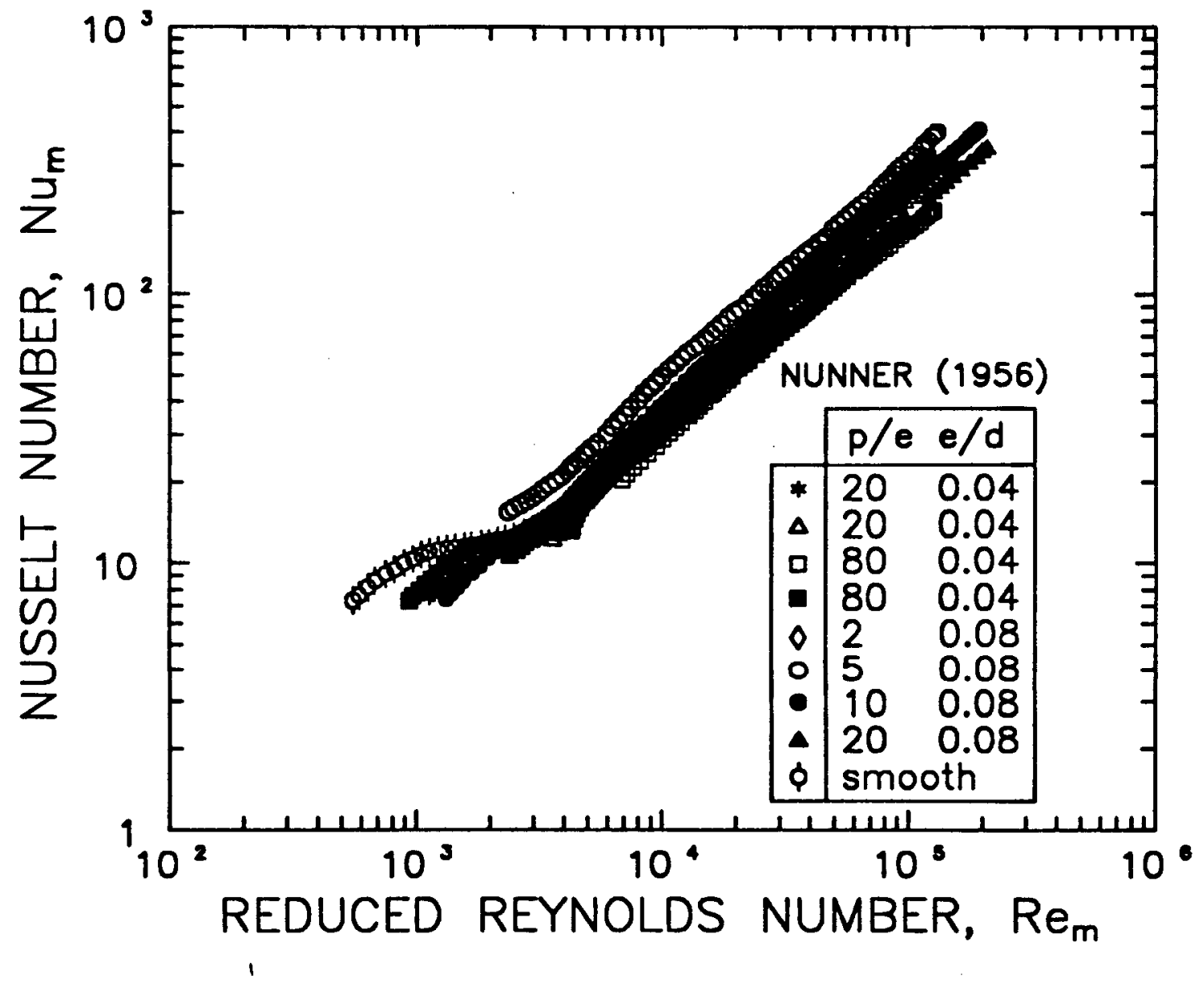

Figure 13. $N u_{m}$ vs. $R e_{m}$ using Nunner's (1956) data. 


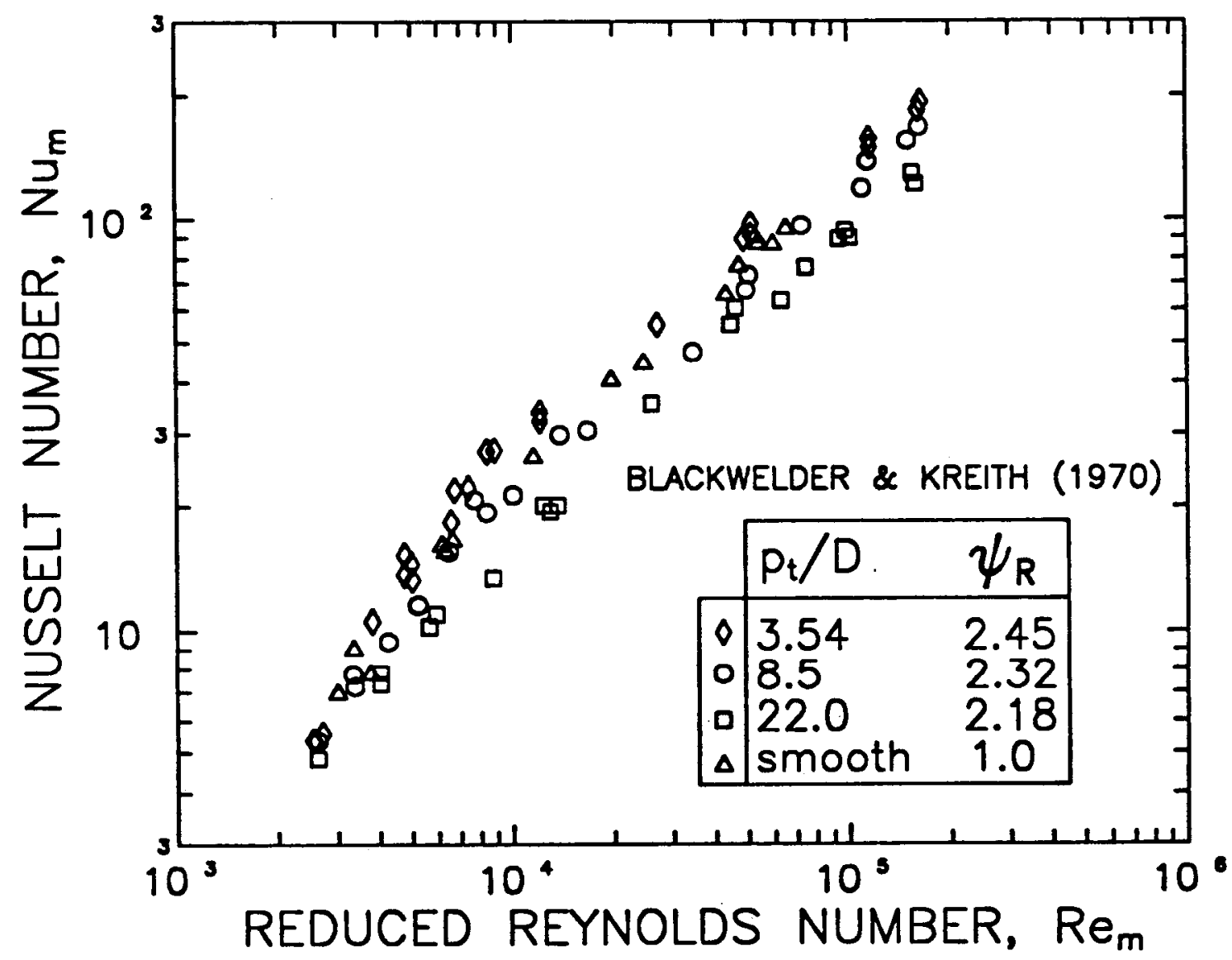

Figure 14. $N u_{m}$ vs. $R e_{m}$ using data of Blackwelder and Kreith (1970). 


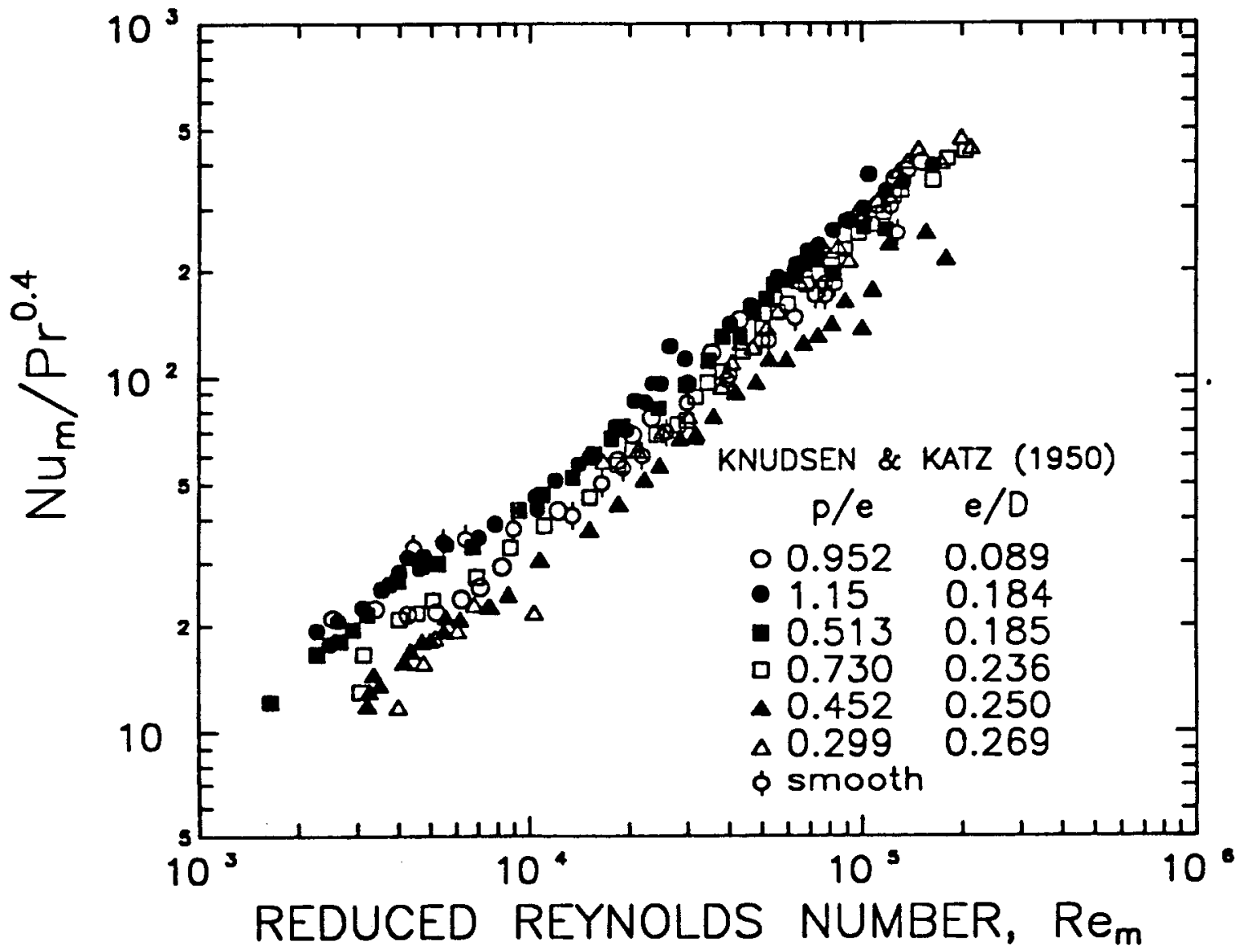

Figure 15. $\left(N u_{m} / P^{0.4}\right)$ using data of Knudsen and Katz (1950). 
Smith and Gowen (1965) reported very limited results for high Prandtl number fluids, but the friction factor trend does not appear to be demonstrative.

Since the smooth duct relative entrance length $\left(L_{e} / D_{e}\right)$ of Knudsen and Katz was 31.0, not sufficiently long to give fully developed flow for their well-rounded entrance, and since the critical $R e$ was about 1770 , it was necessary to establish a reference fully developed flow value for a passage of $\beta=0.278$. Using isothermal friction factor results presented in Fig. 7 of their paper, this reference $R e_{c, r}$ was estimated to be 2989, and it corresponds to the value at which the critical friction factor attains the smooth duct value of 0.008 . It may be noted that the above $R e_{c, r}$ value is not significantly different from that $\left(R e_{c, r}=2873\right)$ determined from the results of Koch and Feind (1958) for $\beta$ $=0.212$ and $L_{e} / D_{e}=50$ (Obot, 1988). The reduced data for the smooth and finned tubes are presented in Fig. 15. In the absence of the $\operatorname{Pr}$ value for each test trial, the ordinate $\left(N u_{m} / \mathrm{Pr}^{0.4}\right)$ is the same as in the original paper. Again, the outcome of the analysis is essentially the same as charted already in Figs. 12-14, with about a $\pm 20 \%$ spread around the authors' smooth tube data.

It is quite evident that, in each of Figs. 12-15, the reduced data lie above and below the smooth tube line. From a careful analysis of the results presented in Koch (1960) for a range of conditions, it appears that the reduced data may lie well below the smooth data for certain values of $R e_{c, a}$. Whether there exists a definite $R e_{c, a}$ range below which the $N u_{m}$ data would deviate markedly from the smooth tube curve, or how far down the Reynolds number scale such a value may occur, is difficult to ascertain from the results available. Suffice it to state that the existence of such a limiting critical Reynolds number would not in any way weaken the general conclusion regarding the decisive role of transition. Bearing in mind that the ratio $N u_{m} / N u_{s}$ will always vary from slightly above to below unity, such information may be useful in the assessment of 
the thermal efficiency of various geometries of roughness.

To shed further light on the connection between transition, friction and heat transfer, the results of several investigators are presented differently on Figs. 16-18. The first of these is a plot of $\phi_{h, \max }=\left(N u_{r} / N u_{\iota}\right)_{\max }$ versus the critical parameter $\psi_{R}(=$ $\left.R e_{c, r} / R e_{c, a}\right)$, while $\phi_{h, \max }$ is plotted against $\phi_{f}\left(=f_{m} / f_{m, s}\right)$ on Fig. 17. On Fig. 18 the ordinate $\left(\phi_{h, \max } / \phi_{f}\right)$ is equivalent to the so-called efficiency index (Webb et al., 1971; Gee and Webb, 1980), with $\psi_{R}$ as the abscissa. Naturally, since the smooth duct friction factor $\left(f_{m, s}\right)$ decreases with $\boldsymbol{R} e_{m}$ for turbulent flow, with a nearly constant $f_{m}$ for a given set of roughness geometry, an arbitrary value of $R e_{m}=50,000$ was used for calculations of $\phi_{f}$. In going from $R e_{m}=50,000$ to $R e_{m}=100,000, f_{m, s}$ changes by no more than $20 \%$; hence the use of the latter $R e_{m}$ value does not modify the observations to be drawn from these figures. To generate the results of Watkinson et al. which are given in Fig. 16, the turbulent flow heat transfer data of an earlier study (Watkinson et al., 1973) were matched tube by tube with the data of Table 1. Although the earlier study was carried out with water, the use of isothermal $\psi_{R}$ data obtained on the same tubes with oil (Table 1) is not expected to alter the general picture to any significant extent (Dodge and Metzner, 1959). Since $N u_{m} / N u_{s} \rightarrow 1$ (Figs. 12-15), it was considered more practical to define $\phi_{h, \max }$ using $N u_{r}$ and $N u_{s}$.

The general picture on each of Figs. 16-18 may appear somewhat confused at first, but such confusion almost disappears as soon as the results of any particular study are viewed separately. Thus, with respect to the results of Koch or Knudsen and Katz, the trend on Fig. 16 supports the view that $\phi_{h, \max }$ increases with increasing $\psi_{R}$, paralleling the trend established in Fig. 3 for the variation of $\psi_{R}$ with $e / D$. Another consistent trend is that the various finned surfaces give $\phi_{h, \max }$ and $\phi_{f}$ values that are generally lower than those with transverse repeated-rib roughness. Also, although there is no 


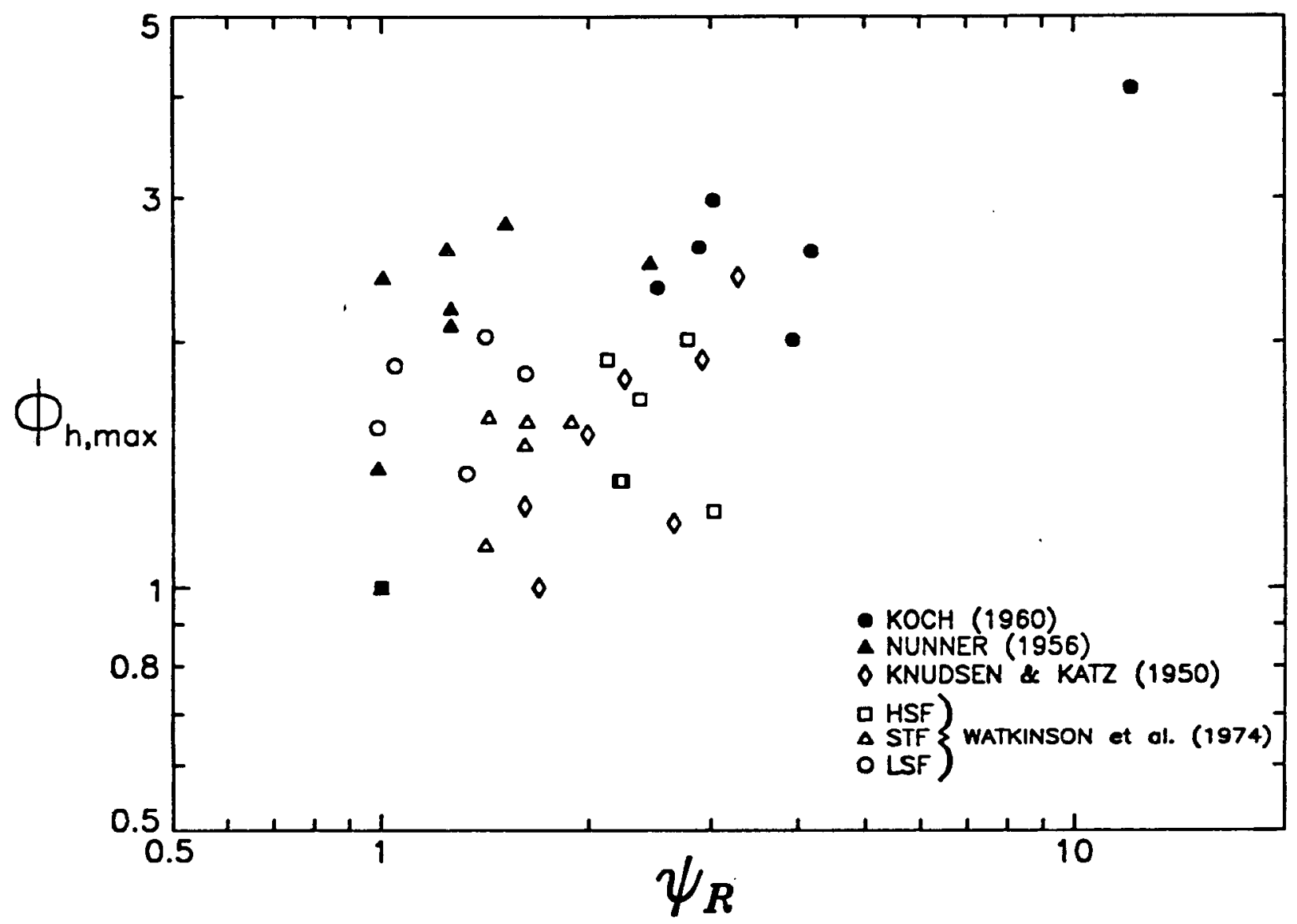

Figure 16. Maximum enhancement factor, $\Phi_{h \max }$ vs. $\psi_{R}$. 


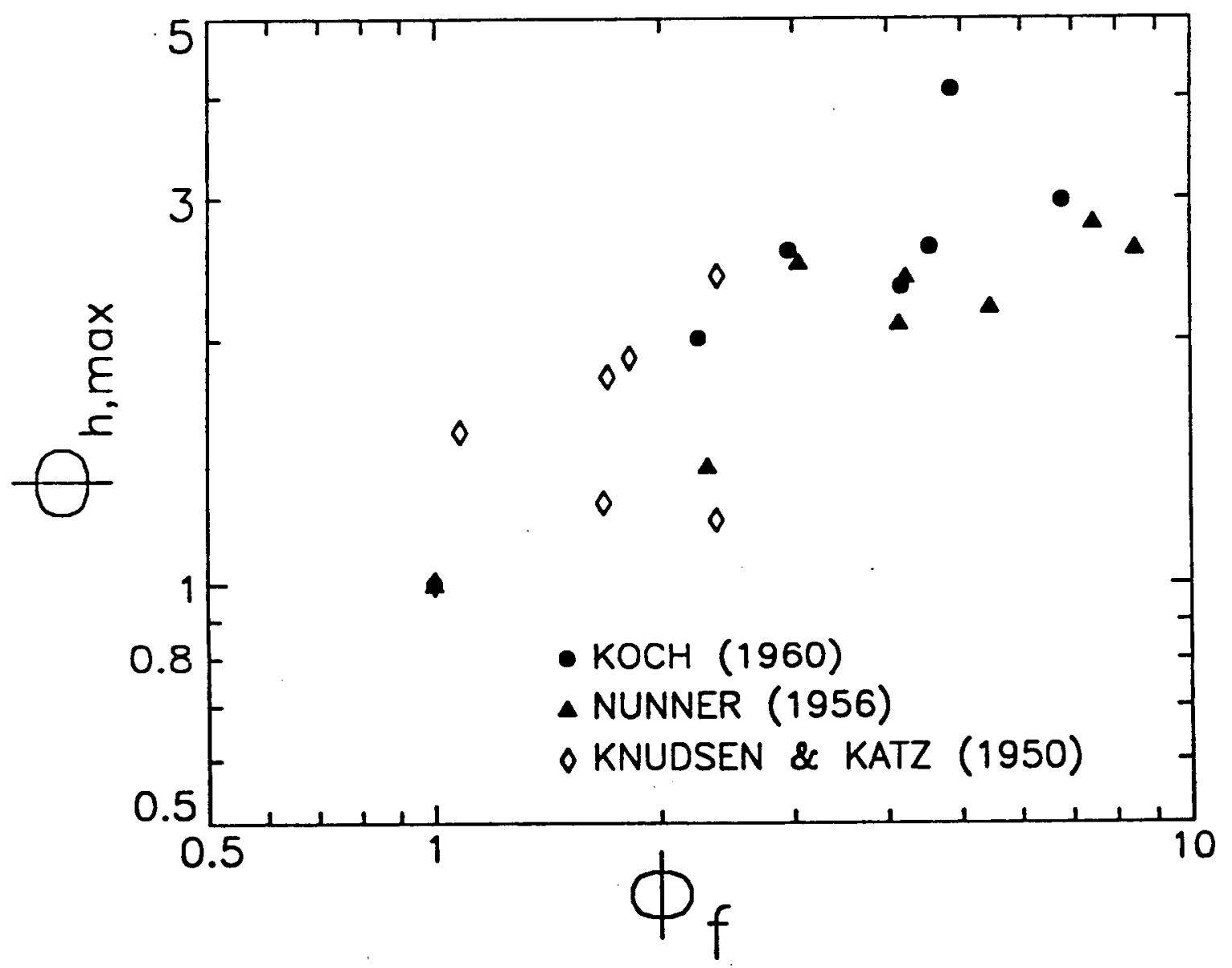

Figure 17. Variation of $\Phi_{h_{m} \max }$ with $\Phi_{f}\left(=f_{m} / f_{m s}\right)$. 


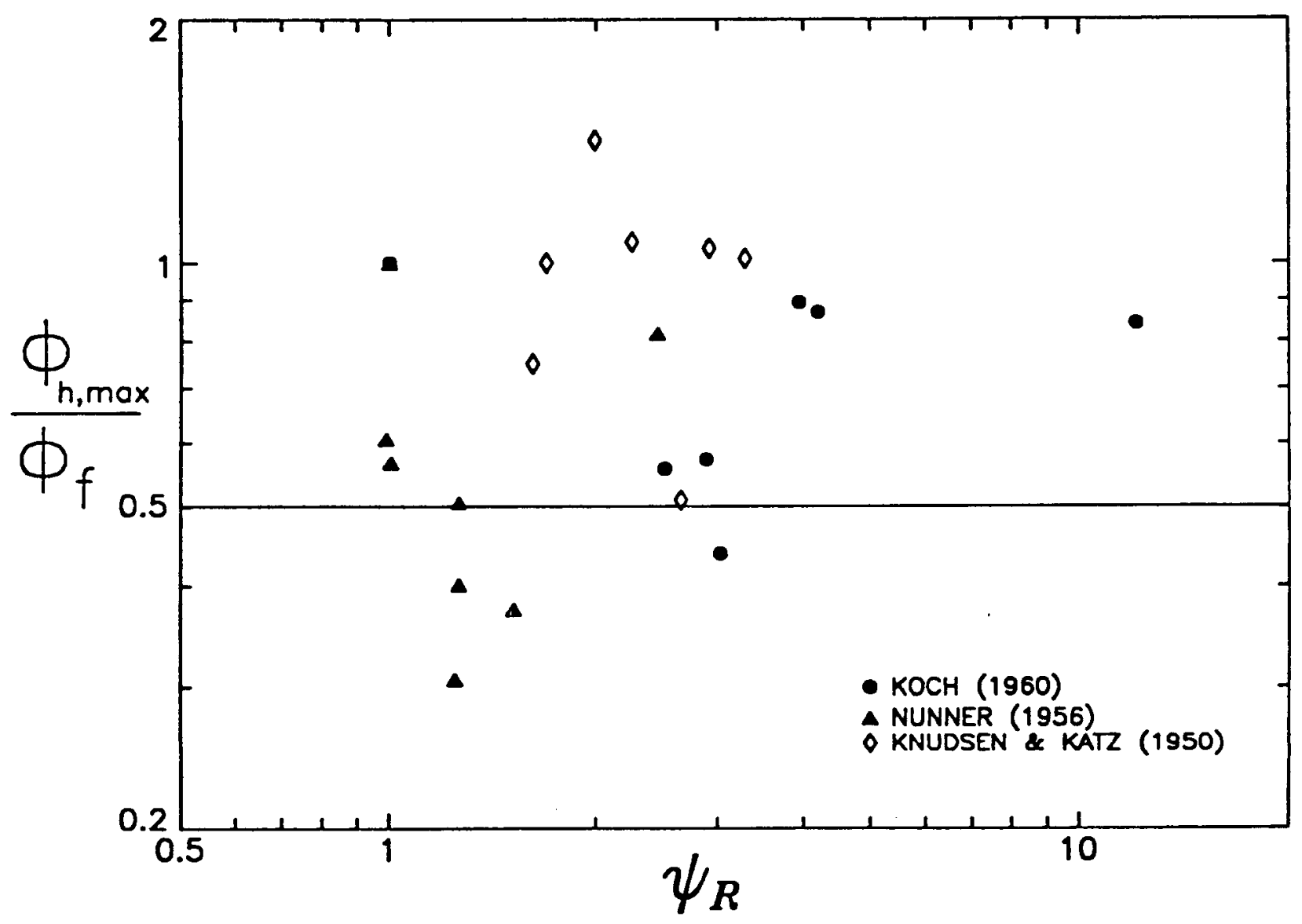

Figure 18. Plot of $\left(\Phi_{h, \text { max }} / \Phi_{f}\right)$ vs. $\psi_{R}$. 
clear distinction between high (HSF) and low (LSF) spirally finned tubes of Watkinson et al., the data suggest that either of these is superior to high straight (STF) finned tubes. It should be emphasized that, unlike the definite trend exhibited by the data of Knudsen and Katz for tests on five ducts with $L_{e} / D_{e} \geq 41$ and one with $L_{e} / D_{e}=33$ (Table 1), the results of Watkinson et al. are marred by a residual effect of $L_{e} / D_{e}$.

Whereas Fig. 16 reinforces the closeness between the $\phi_{h, \text { max }}$ values of Nunner and those of Koch as well as the possible measurement errors of the critical Reynolds number for rough tubes, as discussed in Section 2.2, Fig. 17 shows that any such errors are almost cancelled out when the results are presented in terms of $\phi_{h, \max }$ and $\phi_{f}$. It is pertinent to note that, since Nunner defined the resistance coefficients similarly for both smooth and rough tubes, the ratio $\left(\phi_{f}\right)$ so formed is independent of his definition and is analogous to the usual friction factor ratios. It is quite remarkable that, with the present method, it has been possible to compare two of the most important studies in the literature.

Figs. 17 and 18 show some scatter, but nothing that is unexpected considering the differences between the three studies, the lack of geometrically similar test conditions and of knowlegde of the effect of heat transfer on transition. It is of considerable importance to note that $\phi_{h, \max }$ attains a value of no more than 5 for $1<\psi_{R} \leq 12$ and $1<\phi_{f}<9$ (Figs. 16 and 17). As to whether there should be a unique relation between $f_{m}$ and $N u_{m}$ or between $\phi_{h, \max }$ and $\phi_{f} ;$ although no relation of a universal nature can be established from Fig. 17, the trend on this figure clearly suggests that a definite relation might result from systematic measurements.

From Fig. 18 a condition of remarkable simplicity can be stated as follows:

$$
\phi_{h, \max } \geq \frac{1}{2} \phi_{f} \text { or } \frac{\phi_{f}}{\phi_{h, \max }} \leq 2 \text {. }
$$

This condition, which bears a striking similarity to the well known Reynolds analogy 
except of course for the sign, is satisfied by almost all but four data points. Three of these are Nunner's data, and this is most likely due to errors in $\psi_{R}$ (since $\phi_{f}=f_{m} / f_{m}$, and $\left.f_{m}=\psi_{R} f_{a}\right)$. The other point, due to Koch, does not lie far below the 0.5 line. It is of some interest to note that, in terms of the reduced parameter $\left(\phi_{h, m}=N u_{m} / N u_{s} \rightarrow 1\right)$, the above condition is: $\phi_{h, m} / \phi_{f} \geq 1 / 5$ or $\phi_{f} / \phi_{h, m} \leq 5$.

Contrary to the numerous claims regarding the magnitudes of the improvements in heat transfer, the discussion here makes it clear that the maximum increase in heat transfer is limited by $\phi_{f}$, the value of which is determined by the depression of the critical Reynolds number. So, for the first time, a definite upper limit has been established for the ratio $\phi_{f} / \phi_{h, \max }$. It is also especially noticeable that most of the data on Fig. 18 lie in the $0.5 \leq \phi_{h, \max } / \phi_{f} \leq 1$ range. Interestingly, according to the results of Knudsen and Katz, $\phi_{h, \max } \simeq \phi_{f}$ (Fig. 18), which clearly suggests that helically finned tubes may be more effective than transverse repeated-rib roughness for the same $\psi_{R}$. Further studies are clearly needed to establish these trends precisely.

In summary, it is quite evident that the difficulty in reconciling the results of numerous investigators is not to be explained solely by any peculiarity of the duct cross-section or configuration of roughness tested. It has indeed been established that differences among the various results arise from transition, a phenomenon which hitherto has been almost overlooked in the general treatment of friction and convective heat transfer. The presentation in this section has provided at least partial understanding of the connection between transition and heat transfer. The proposed law of corresponding states has the peculiar advantage that it affords, even over the widest range of geometric and flow conditions, a consistent basis for comparison of data obtained on enhanced 
passages.

\subsubsection{Transition-Based Similarity Parameter Vs. Roughness Reynolds Num-} ber.

The friction similarity concept for rough pipes was developed by Nikuradse (1933) and successfully used to correlate his data for sand-grain roughness. For similarly roughened tubes, Dipprey and Sabersky (1963) also derived essentially the same expression and extended the treatment to heat transfer. Thereafter, researchers (Webb et al., 1971; Han et al., 1978; Withers 1980a,b; to mention a few) have correlated their heat transfer data using this concept. For rough passages, it has been the practice to use the roughness Reynolds number $\left(e^{+}\right)$which is given by equation (7) to correlate heat transfer data:

$$
e^{+}=R e_{a}(e / D)\left(f_{a} / 2\right)^{1 / 2}
$$

With the knowledge that the conventional Reynolds number is not a generally valid similarity parameter, as thoroughly documented in the preceding sections, one possible modification to equation (7) is to replace $R e_{a}$ and $f_{a}$ by $R e_{m}=\psi_{R} R e_{a}$ and $f_{m}=\psi_{f} f_{a}$, respectively, to obtain:

$$
e_{m}{ }^{+}=R e_{m}(e / D)\left(f_{m} / 2\right)^{1 / 2}
$$

To fully appreciate whether such a modification can be applied satisfactorily to rough passages, several observations must be made. First, for a given $e / D, f_{m}$ assumes almost a constant value for $R e_{m}>2,100$, hence $e_{m}{ }^{+}$will always be directly proportional to $R e_{m}$. In this regard it is important to note that $f_{m}$ is dependent only on the geometric configurations of roughness for $R e_{m}>2,100$. However, in the laminar regime, $f_{m}$ is inversely proportional to $R e_{m}$ but is essentially independent of $e / D$ or other geometric parameters of roughness; hence $e_{m}{ }^{+} \propto \sqrt{ } R e_{m}$. And, further, since transition appears to 
be the main mechanism which determines friction and heat transfer in passages and since its effects should be almost totally accounted for by introduction of the parameters $\psi_{R}$ and $\psi_{f}$, it should be possible to establish an equivalent form of equation (7) only in terms of $f_{m}$ and $R e_{m}$. These considerations raise considerable doubts about the wisdom of retaining $e / D$ in equation (8).

Since $e^{+}$is obtained by simply grafting on the ratio $e / D$ to the result of a force balance relation, it seems more logical to eliminate $e / D$ totally from equation (8). Using the fact that $2 \pi r \tau_{w}=(d P / d x) \pi r^{2}$ and $f=(d P / d x)\left(D / 2 \rho \bar{V}^{2}\right)$, it is easily established that

$$
R e \sqrt{ }(f / 2)=U_{\tau} D / \nu=R e_{\tau}
$$

where the friction velocity $U_{\tau}=\left(\tau_{w} / \rho\right)^{1 / 2}$ and $\tau_{w}=$ wall shear stress. The so-called roughness Reynolds number is obtained by multiplying equation (9) by $e / D$. Consistent with the general treatment of friction in smooth passages, the modified form of equation (9) which satisfies the basic requirement for flow similarity is given by:

$$
R e_{m} \sqrt{ }\left(f_{m} / 2\right)=R e_{\tau m}
$$

The significance of equation (10) must not be overlooked. In the first place, $R e_{m}$ in turbulent flow is directly proportional to the friction Reynolds number, $R e_{r m}$; an indication that when sets of data are plotted against $R e_{m}\left(=\psi_{R} R e_{a}\right)$ and against $R e_{\tau m}$, the two plots should be fully equivalent, regardless of whether the results were obtained on smooth or rough passages. Second, it reinforces the dependence of the friction velocity and hence the wall shear stress on the critical parameters for transition. Third, it provides a straightforward means for determining the friction Reynolds number from which the wall shear stress can be calculated.

To shed some light on the use of these parameters $\left(R e_{m}, R e_{\tau m}, e^{+}\right.$and $\left.e_{m}{ }^{+}\right)$, one 
approach would be to attempt to correlate results of systematic experiments using each of these definitions. This method is not adopted here for two reasons: first, it obscures the physical situation; and, second, our main interest at this stage is to fully establish the role of transition to turbulence which, hopefully, should lead to better understanding of the transfer processes. Instead, straightforward calculations of the Stanton number ratio $\left(S t_{r} / S t_{s} ; r=\right.$ rough, $s=$ smooth) were made using the results of Koch (1960) for transverse disc-shaped and ring inserts. Note that some of these results were presented as $N u_{m}$ vs. $R e_{m}$ on Fig. 12.

In Figs. 19-24, the heat transfer results of Koch are presented differently for a range of height-to-diameter $(e / D)$ and pitch-to-height $(p / e)$ ratios. Since Koch's study covered moderate as well as unusually high values of values of $e / D$ and $p / e$ (Table 1 ), two distinct trends resulted from the present analysis. Accordingly, and for the sake of clarity, Figs. 19-22 show results for moderate geometric parameters while those for high $e / D$ and $p / e$ are given in Figs. 23 and 24. For Figs. 19-22, the abscissa corresponds to $R e_{m}, R e_{r_{m}}, e_{m}{ }^{+}$and $e^{+}$, successively, while plots of Stanton number ratio versus $R e_{m}$ and $R e_{\tau m}$ are presented in Figs. 23 and 24, respectively. To compute $R e_{m}, R e_{\tau m}$ or $e_{m}{ }^{+}$, the appropriate critical parameters and the $f_{m}$ data in Section 2.2 were used.

To begin the discussion, attention may be turned to Figs. 19-22 where comparison from figure to figure reveals one basic difference between $R e_{m}$ or $R e_{\tau m}$ and $e^{+}$or $e_{m}{ }^{+}$. Fig. 19 shows that $S t_{r} / S t_{s}$ is almost independent of $e / D$ or $p / e$ for $R e_{m}<6,000$, a fact that is also strikingly illustrated in Fig. 20 for $R e_{\tau m}<1,000$. By contrast, the effect of $e / D$ is quite evident from the comparison of the $p / e=9.8$ results for $e / D=0.045$ and 0.075 (Figs. 21 and 22). In the case of $e^{+}$, the trend on Fig. 22 is consistent with previous results (Webb et al., 1971; Dawson and Trass, 1972). Clearly, there is no advantage to using $e_{m}+$ instead of $e^{+}$. This arises from the fact that, although $R e_{m}$ is greater than $R e$, 


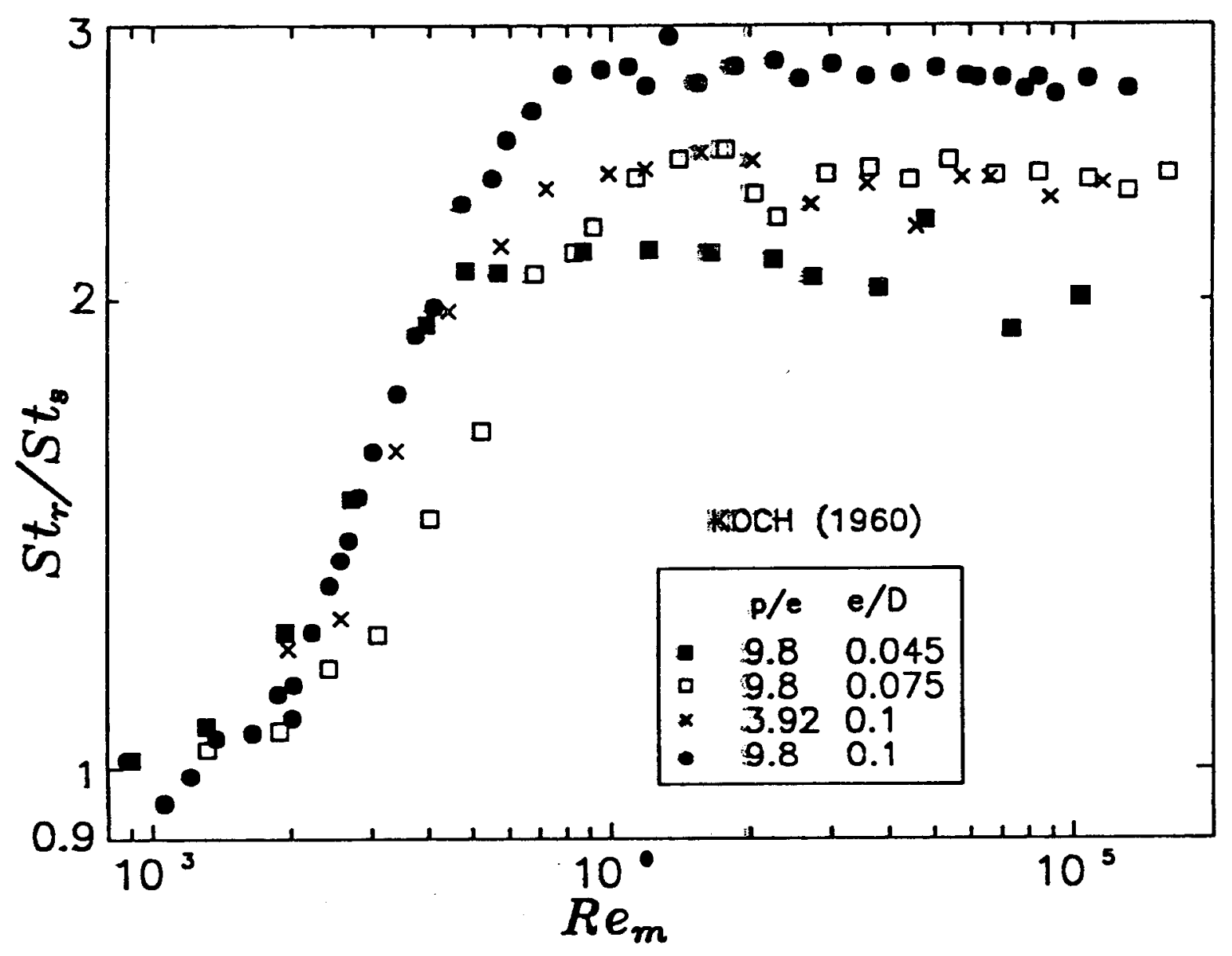

Figure 19. Stanton number ratio vs. $R e_{m}$. 


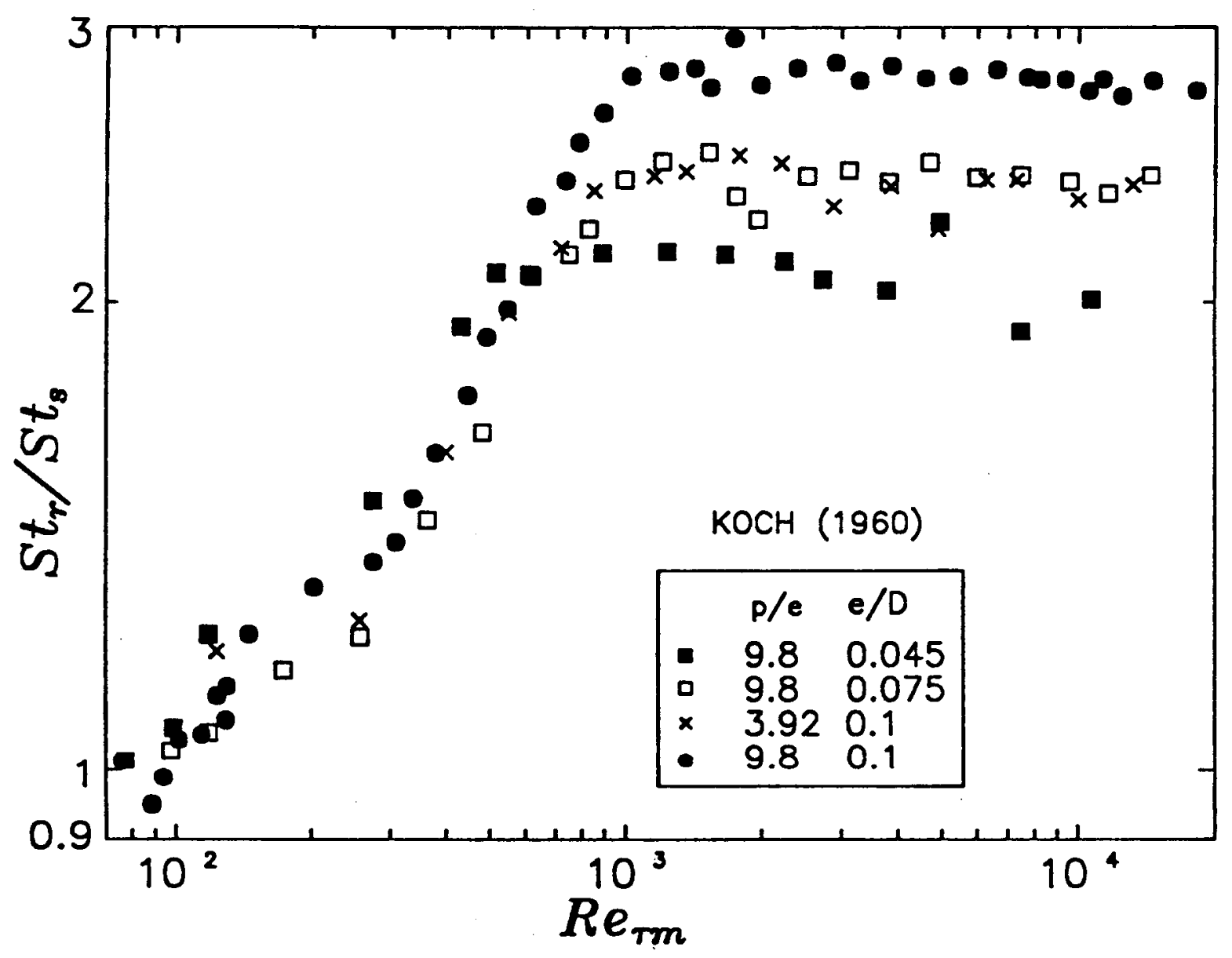

Figure 20. Stanton number ratio vs. $R e_{r m}$. 


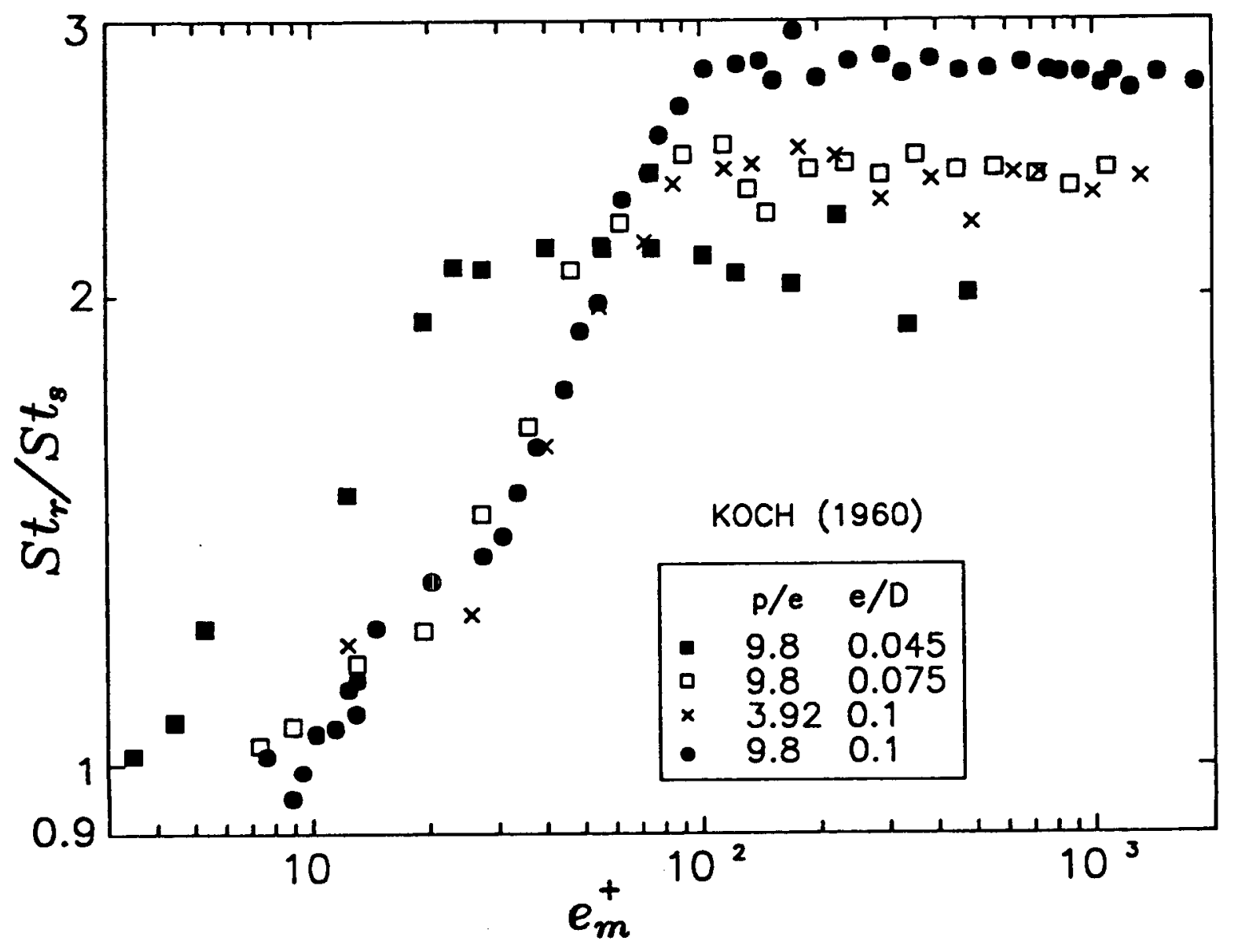

Figure 21. Stanton number ratio vs. $e_{m}{ }^{+}$. 


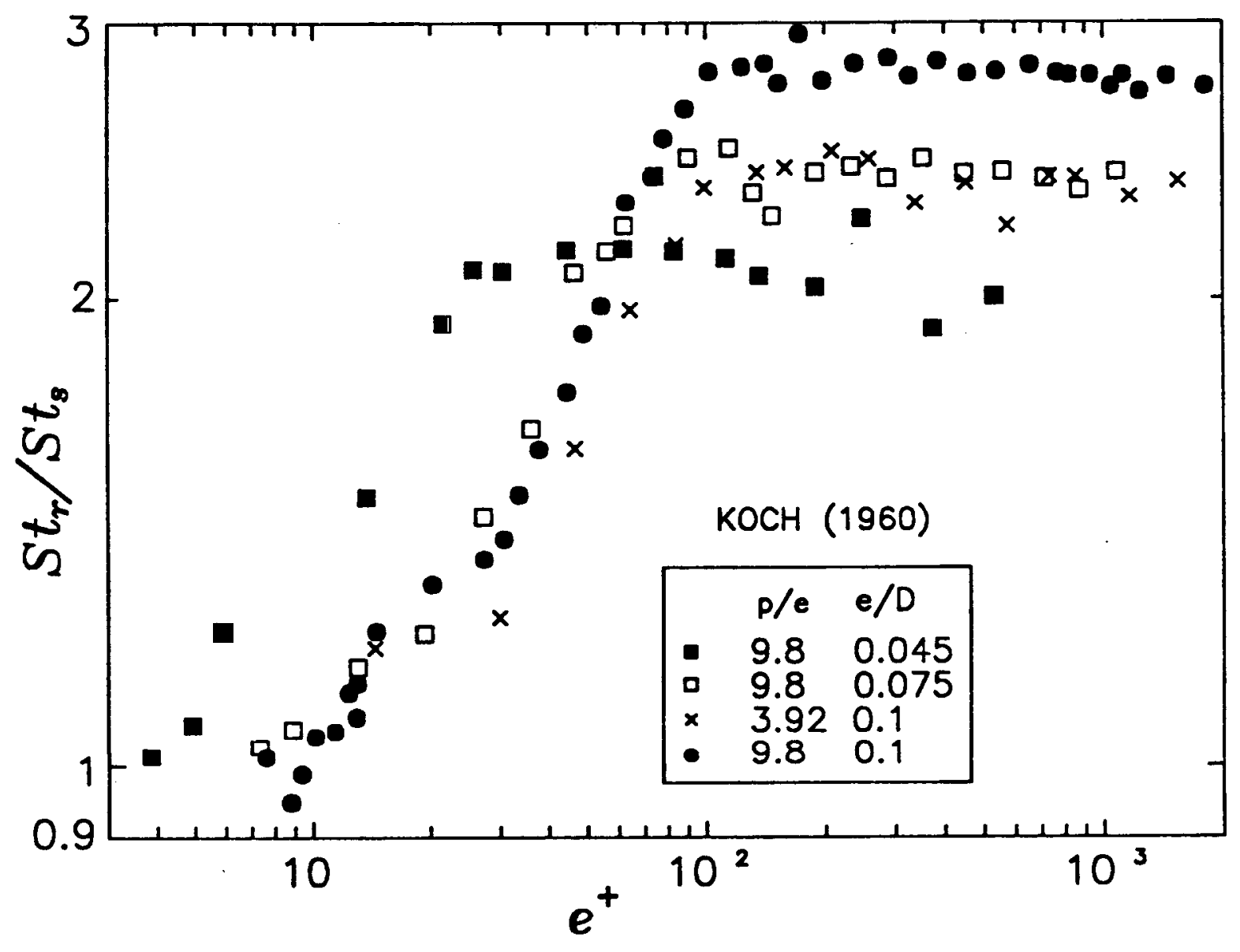

Figure 22. Stanton number ratio vs. $e^{+}$. 
$f_{m}$ is generally lower than the conventional friction factor $f_{r}$. The result is that there are no large differences in the calculated values between $e_{m}{ }^{+}$and $e^{+}$. Thus, Fig. 21 is nearly identical to Fig. 22. Given the general trend displayed here in Figs. 19-22, it is difficult to make a strong case in favor of the continued use of the conventional roughness Reynolds number. On the contrary, the use of the modified friction Reynolds number has the peculiar advantage that, over a wide range of geometric conditions, the reduced data are almost generalized.

Of the common features on Figs. 19-22, one is that a profile for $S t_{r} / S t$, passes through a maximum in some range of $R e_{m}, R e_{\tau m}, e_{m}{ }^{+}$or $e^{+}$. In the case of $R e_{m}$, it is especially noticeable that this maximum occurs between $R e_{m}=5,000$ and 15,000 which, expressed in terms of the conventional Reynolds number $(R e)$, corresponds to between $R e=3,000$ and 7,000 . For $S t_{r} / S t_{s}$ vs. $e^{+}$, the existence of peak values in these profiles can be inferred from the results of the two studies that are cited in the preceding paragraph. Another feature is that, beyond the locations of the peak values, the spread around a mean line is on the order of $25 \%$. And, further, although the effect of $e / D$ is more pronounced in Fig. 21 or 22 than on Fig. 19 or 20, it is quite evident that $S t_{r} / S t_{\text {s }}$ is almost independent of $p / e$ up to $e_{m}{ }^{+}$(or $e^{+}$) $=1,000$.

In Figs. 23 and 24, which show results obtained with large values of $e / D$ and/or $p / e, S t_{r} / S t_{s}$ values are plotted against $R e_{m}$ and $R e_{r m}$, respectively. With these data, since the effects of $e / D$ were even more pronounced in plots using $e_{m}{ }^{+}$and $e^{+}$than those on Figs. 21 and 22, such plots are not given. As with Figs. 19 and 20, Figs. 23 and 24 are completely similar. It is of some interest to note that, in sharp contrast to the trends on Figs. 19-22 for moderate geometric parameters, the results on Fig. 23 or 24 show no definite maximum. In Section 3.2, it was noted that, for some range of geometric conditions, the reduced data of Koch were closely approximated by the 


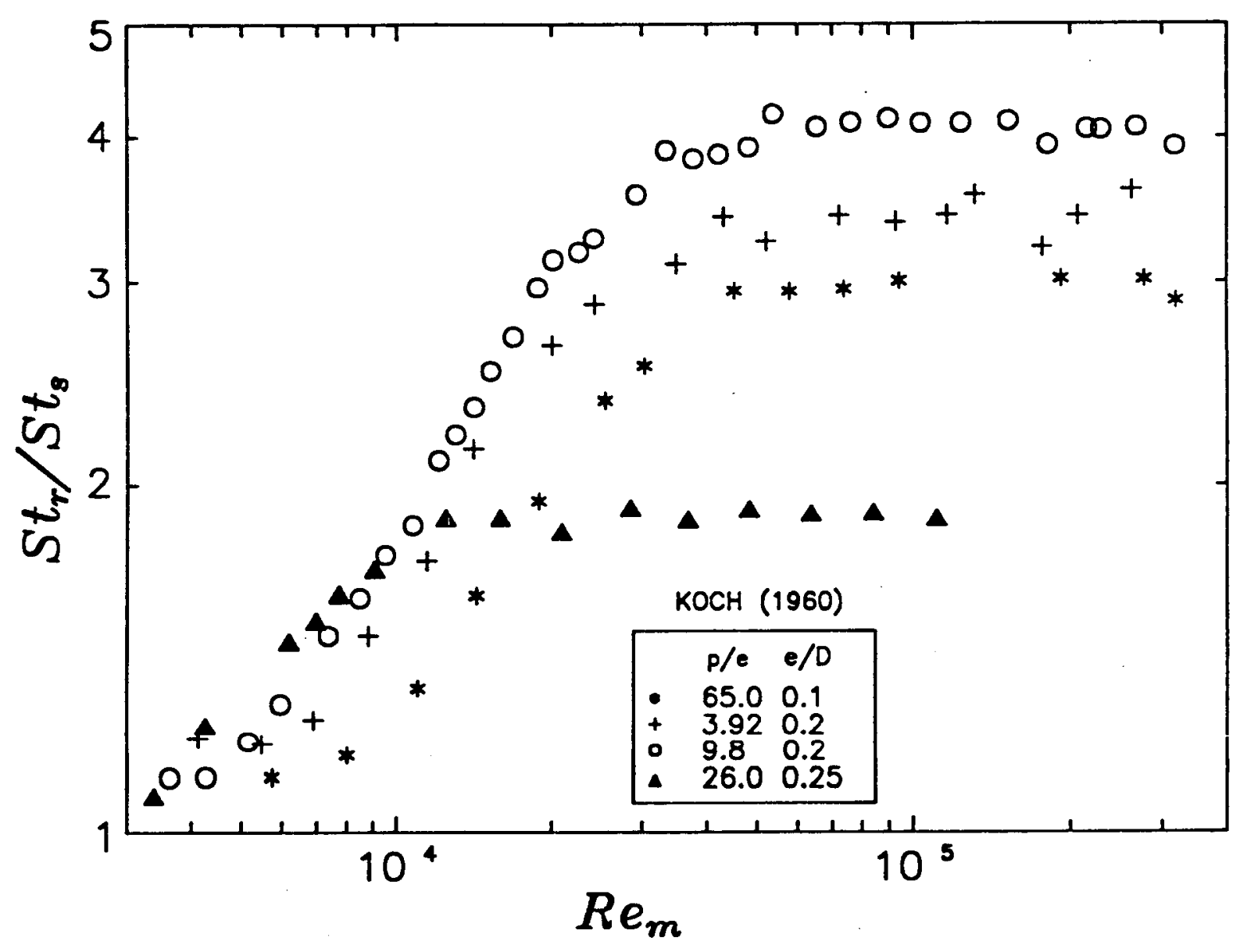

Figure 23. Stanton number ratio vs. $R e_{m}$. 


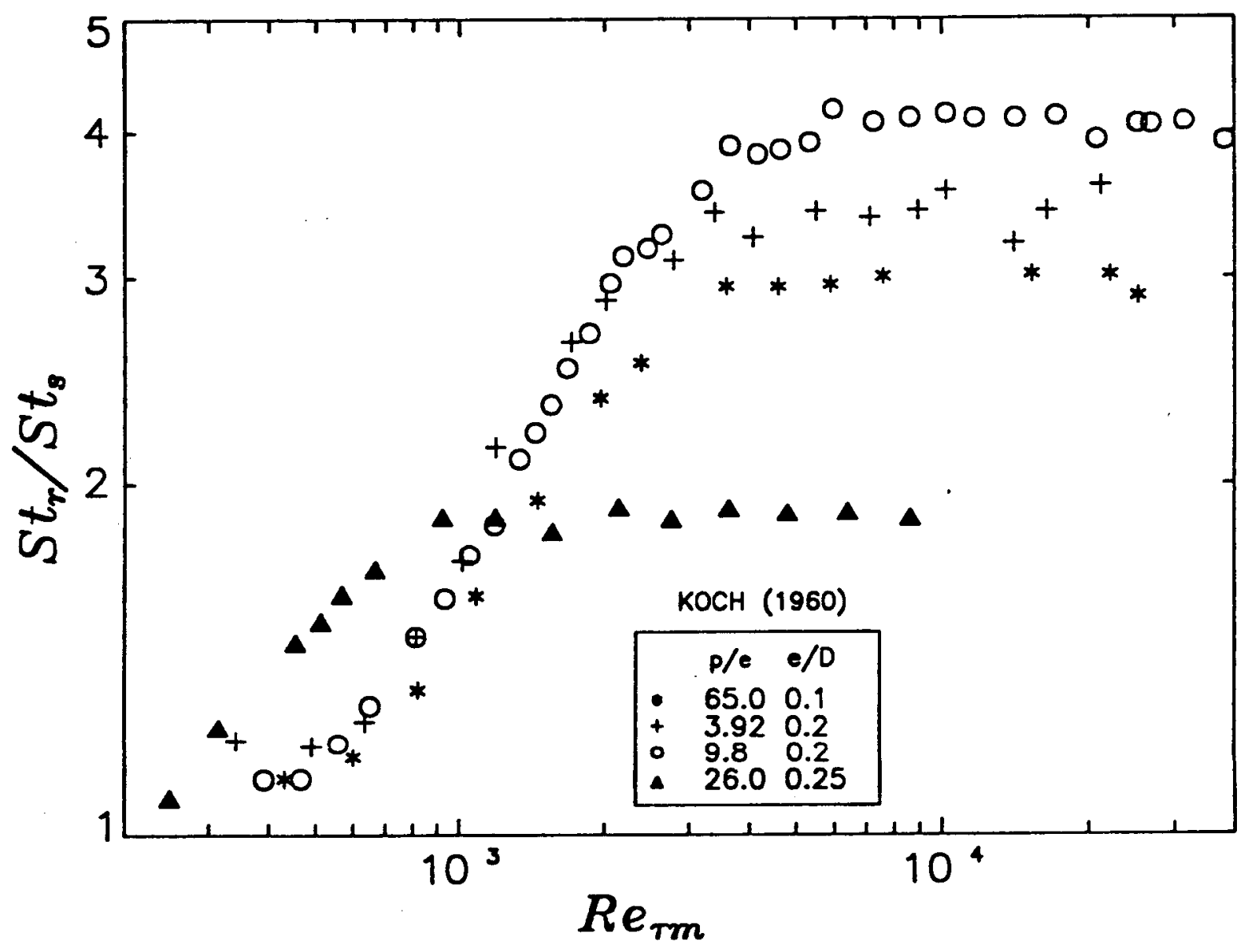

Figure 24. Stanton number ratio vs. $R e_{\boldsymbol{r m}}$. 
smooth tube relation (Fig. 12), but that such a resolution was not realized for some of the test conditions. This fact is seen more clearly here from comparisons between Figs. 19 and 23 or Figs. 20 and 24.

From the aforegoing comparison and discussion, it is quite clear that the choice is between $R e_{m}$ and $R e_{\tau m}$. Of these two, $R e_{m}$ is much to be preferred for two reasons. The first relates to simplicity, inasmuch as the critical Reynolds numbers $\left(R e_{c, r}\right.$ and $\left.R e_{c, a}\right)$ and $R e_{a}$ are all that are needed to compute $R e_{m}$. By contrast, to compute $R e_{\tau m}$, all critical parameters $\left(R e_{c, r}, R e_{c, a}, f_{c, r}\right.$ and $\left.f_{c, a}\right)$ as well as $f_{a}$ and $R e_{a}$ must be known. A second reason concerns engineering practice. Since $R e_{m}$ is simply the product of the conventional Reynolds number and the ratio of the critical Reynolds numbers, there is absolutely no difficulty with its use in practice. Given the amount of information that is needed to compute $R e_{\tau m}$, it is can be readily appreciated that the same is not true of $R e_{\tau m}$

In summary, this comparative evaluation reinforces the significance of the corresponding states law as well as the role of transition in determining heat transfer in enhanced tubes. Specifically, it has been demonstrated that, in terms of $R e_{m}$ or $R e_{\tau m}$, the Stanton number ratios for a range of $p / e$ and $e / D$ can be represented by a single regression line. Also, there are clear indications that the Stanton number ratio profiles attain peak values over the range of Reynolds numbers between 3,000 and 7,000 for small and moderate values $p / e$ and $e / D$.

\section{FLUID HEATING VERSUS COOLING}

Certainly, an analysis of the role of transition on heat transfer will be incomplete without a discussion of the effects of heating and cooling of a fluid for several reasons. First, just as the Reynolds number arises naturally from dimensional analysis on the 
equations of motion, a similar treatment of the energy equation gives rise to the Prandtl number, $\operatorname{Pr}$. Second, it is widely reported in the literature that the dependence of Nusselt or Stanton number on $\operatorname{Pr}$ varies according to whether the fluid is being heated or cooled. Third, in addition to the $\operatorname{Pr}$ dependence, many correlations include a viscosity (or temperature) ratio as suggested by Sieder and Tate (1936) based on an analysis of oil data for $R e \leq 2100$. That the validity of the Reynolds number similarity concept is determined by transition was demonstrated for friction (Obot, 1988) and reinforced here. It is, therefore, natural to suppose that a similiar situation might occur for Prandtl number.

Although this presentation is intended to provide information that is little more than qualitative, due largely to lack of experimental data for tubes, it should nevertheless be sufficient to show that the limit of stability, and hence the critical Reynolds number, depends on whether a fluid is being heated or cooled. Of considerable importance to the present consideration is the well known fact that the stability of a boundary layer is determined largely by the curvature of the velocity profile at the wall. At the wall, the streamwise equation of motion reduces to:

$$
\mu_{w}\left(\frac{d^{2} U}{d y^{2}}\right)_{w}=-\left(\frac{d U}{d y}\right)_{w}\left(\frac{d \mu}{d y}\right)_{w}+\frac{d p}{d x}
$$

Using the fact that $(d U / d y)_{w}=\tau_{w} / \mu_{w}$ and $\tau_{w}=(d p / d x)\left(A / P_{w}\right)$, equation (11) becomes:

$$
\left(\frac{d^{2} U}{d y^{2}}\right)_{w}=-\frac{1}{\mu_{w}} \frac{d p}{d x}\left[\frac{A}{P_{w} \mu_{w}}\left(\frac{d \mu}{d y}\right)_{w}-1\right]
$$

Since $\left(A / P_{w} \mu_{w}\right)(d \mu / d y)_{w} \gg>1$, at least for air and water, equation (12) can be approximated by:

$$
\left(\frac{d^{2} U}{d y^{2}}\right)_{w}=-\frac{A}{P_{w} \mu_{w}^{2}} \frac{d p}{d x}\left(\frac{d \mu}{d y}\right)_{w}
$$

When a gas is heated $\left(T_{w}>T_{f}\right):(\partial T / \partial y)_{w}<0,(d \mu / d y)_{w}<0$, and the curvature of the velocity profile is positive. On the other hand, $(\partial T / \partial y)_{w}<0,(d \mu / d y)_{w}>0$ and 
$\left(d^{2} U / d y^{2}\right)_{w}$ is negative for a liquid. It follows that the limit of stability or the critical Reynolds number may be higher for a liquid than for a gas when $T_{w}>T_{f}$. However, since viscosity decreases as the temperature rises for liquids, but increases with increasing temperature for gases, it is quite evident that the above situation is reversed when $T_{w}<T_{f}$, i.e., the limit of stability for a liquid is lower than for a gas. These observations, which are definitely confirmed by numerical calculations on boundary layers with air and water by Cebeci and Smith (1968) and Wazzan et al. (1968) respectively, should also be applicable to flows in channels. The calculations by the latter also indicated that the critical Reynolds number increased markedly with $T_{w}$, reached a maximum around $T_{w}=60^{\circ} \mathrm{C}$ with $T_{f}=15.6^{\circ} \mathrm{C}$, and decreased gradually as $T_{w}$ was increased beyond the maximum value.

From the above discussion and that in Section 3.2, the general picture can be summed up as follows: average heat transfer coefficients should be higher for heating than cooling of gases while heating of a liquid should give coefficients that are lower than for cooling. This observation is not entirely consistent with the usual empirical treatment which, with a fixed exponent on Reynolds number, gives $N u \propto \operatorname{Pr}^{n}$, where $n$ $=0.4$ and 0.3 are widely quoted for heating and cooling, respectively. In this regard, the existing literature on heat transfer in channels is not particularly useful partly because of the almost total neglect of the laminar and transition regions and partly because, even when measurements did cover these regions, the results are not sufficiently accurate to permit precise determination of the critical parameters. From a careful study of the available results, the following observations can be made. For water (Knudsen and Katz, 1950), the critical Reynolds number with heating can be significantly higher than without heating, a trend that is consistent with stability calculations. For air, whereas the inference from the results of Koch or Nunner is that heating can lower the critical 
$R e$, as might be expected, Washington and Marks (1937) reported exactly the opposite trend. Clearly studies with liquids and gases are needed before the correct picture can be established.

It must not be inferred, however, that if $\tau_{w}=-\left(A / P_{w}\right)(d p / d x)$, the conclusions drawn above will remain unchanged, for obviously, since the curvature of the velocity profile is controlled by the pressure gradient, these observations will be modified when there is a favorable $(d p / d x<0)$ gradient. Thus, when $\tau_{w}=-\left(A / P_{w}\right)(d p / d x)$, heat transfer coefficients should be greater for cooling than for heating of a gas, while the trend should be exactly the opposite for liquids. So, the effect of heat transfer on transition, a problem which has received very meager treatment in the past, is quite complicated and certainly deserves further studies.

In conclusion, our view is that the dependence of heat transfer coefficient on Prandtl number, if any, and like that on flow velocity, should be definite for both heating and cooling. Any departure from such a unique Prandtl number effect, such as the inclusion of viscosity or temperature ratio, has its origin in the differences in heat transfer coefficients that are brought about by variations in the critical parameters for transition. In this regard, the reader is probably aware of the fact that, even in the case of smooth passages, a temperature (or viscosity) ratio is often used to reconcile differences between isothermal and nonisothermal friction factors (Campbell and Perkins, 1968). As with heat transfer, this is a manifestation of the influence of transition, for obviously differences in friction factors can only be brought about through changes in the onset of transition. 


\section{DISCUSSION}

In this report, efforts have been made to clarify and to explain the role of transition in determining friction and convective heat transfer in flow passages. That transition has a decisive influence on convective heat transfer and certainly paints a good picture of the process should not be altogether surprising. In the first place, it has been documented in virtually every text on the subject (see, for example, McAdams, 1942) and it is almost of universal knowledge that a marked increase of heat transfer coefficient with flow velocity occurs only after the onset of transition. In other words, as with friction, there are two fundamentally different trends for heat transfer due to transition. And, second, studies on the effect of free stream turbulence indicate that an increase in turbulence intensity causes earlier transition and this appears to be the physical phenomenon responsible for the corresponding increase in heat transfer coefficients (Kestin and Maeder, 1957; Kestin et al., 1961). Indirect support for this view comes from the work of Dryden (1953) who carefully re-analyzed the critical Reynolds number results for a single roughness element on a flat plate at zero incidence and different free stream turbulent intensities. He showed that, when plotted as $R e_{c, r} / R e_{c, s}\left(c, r=\right.$ rough, $c, s=$ smooth) versus $e / \delta_{1}$ $\left(\delta_{1}=\right.$ boundary layer displacement thickness at the element), the results with different intensities of turbulence were completely generalized, being a function of the single parameter $e / \delta_{1}$. So, the effect of roughness on transition is analogous to that of free stream turbulence. Strangely enough, although a lot is known about transition, there has been almost no systematic analysis of its role on heat transfer, and, until recently, no general treatment of its influence on friction has been made.

The numerous observations on the dependence of heat transfer coefficients on the critical parameters for smooth and rough passages can best be integrated with the help of Fig. 25. This figure contains the same information as Fig. 5 but with one exception, that 


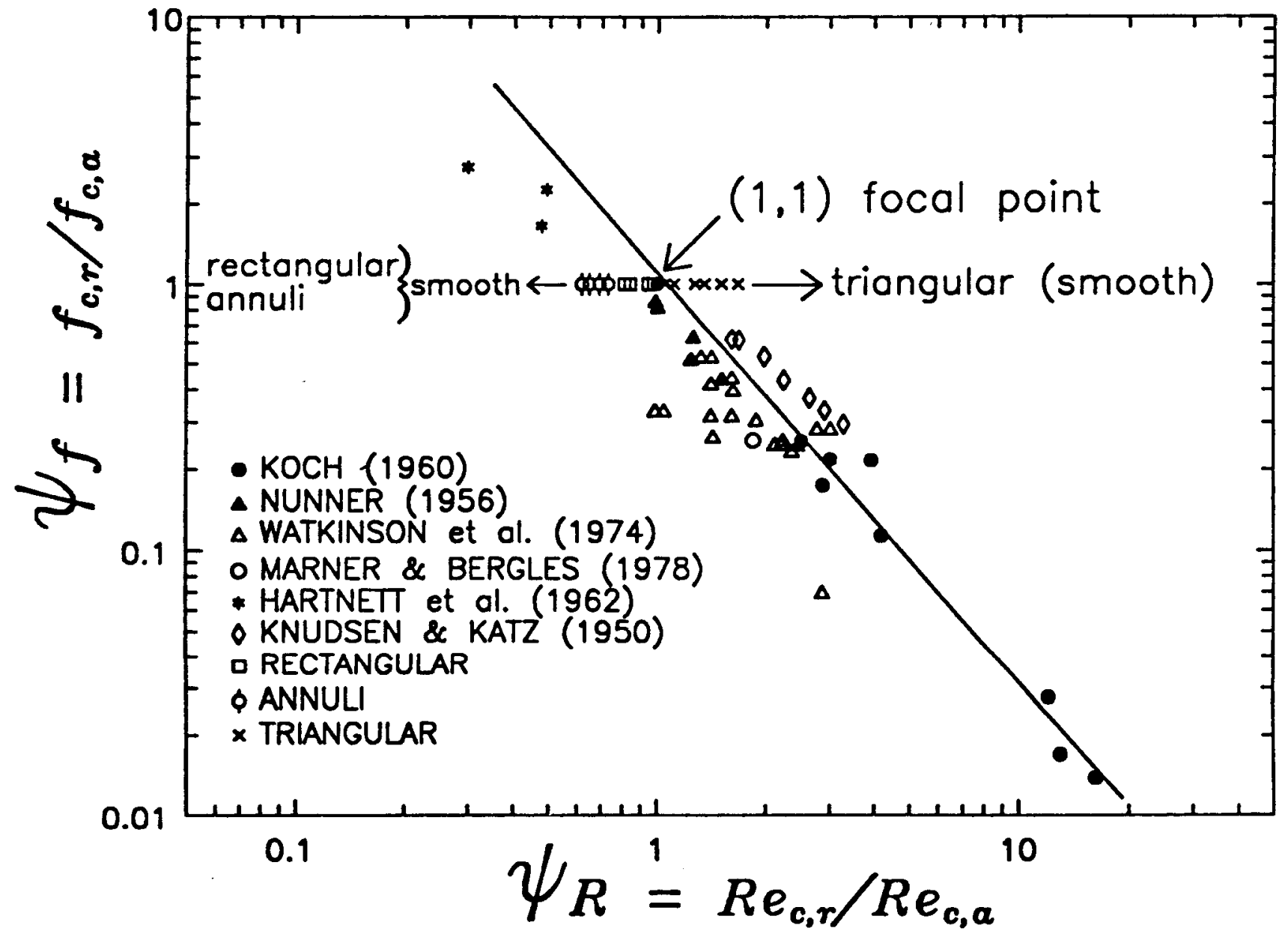

Figure 25. Generalized plot of $\psi_{f}$ vs. $\psi_{R}$ for smooth and rough passages. 
is, the inclusion of a few results for smooth rectangular, annular and triangular passages as well as the three data points obtained by Hartnett et al. (1962) with a smooth entrance. This additional information was determined from tabulations in a previous paper (Obot, 1988). Since $\psi_{f}\left(\right.$ i.e. $\left.f_{c, r} / f_{c, a}\right)$ is almost unity for all smooth passages, these data are located along $\psi_{f}=1$. For clarity, the data have not been identified according to the aspect, diameter or height-to-base ratio. It is important to note, however, that the focal point on this figure is $\left(\psi_{f}, \psi_{R}\right)=(1,1)$ and this corresponds to a reference condition with $f_{c, r}=0.008, R e_{c, r}=2,100$. The aspect (or diameter) ratio of the smooth passage is increasing with decreasing $\psi_{R}$, while the triangular duct height-to-base ratio is increasing monotonically with $\psi_{R}$, from this focal point.

In terms of $\left(\psi_{f}, \psi_{R}\right)$, the parametric description of the heat transfer process amounts to this: heat transfer coefficients are higher than smooth circular tubes for $\psi_{f}<1$ and $\psi_{R}>1$, while the trend is exactly the opposite for $\psi_{f}>1$ and $\psi_{R}<1$. It is especially noticeable that smooth triangular passages produce an effect on transition $\left(\psi_{R}>1\right)$ which is similar to that due to roughness, fully supporting the conclusions in Section 3.1 on the smooth duct heat transfer trends. In this report, the situations: $\psi_{f} \leq 1, \psi_{R} \geq 1$ have been addressed satisfactorily. The rather unique condition, $\psi_{f}>1$ and $\psi_{R}<1$, is a special case and this corresponds to the situation where special precautions are taken to delay transition. Relative to the reference, $\left(\psi_{f}, \psi_{R}\right)=(1,1)$, no improvements in heat transfer should be expected for $\psi_{f}>1$ and $\psi_{R}<1$, and this has been confirmed by numerous results (Liu et al., 1988; Matthys et al., 1987). We are therefore led to the first general law of convective heat transfer which, simply stated, amounts to this: to alter the existing heat transfer for a given set of conditions, be it in the direction of higher or lower rates, it is necessary to change the critical Reynolds number or critical friction factor for transition. In passing, it is of interest to note that the data of Hartnett et al. 
for a very smooth entrance do not lie far from the line obtained by the extrapolation of equation (3), suggesting that, if systematic measurements were made, a unique relation between $\psi_{f}$ and $\psi_{R}$ may exist for all situations where the onset of transition is effected artificially.

In general, the conditions of the local flow over roughness elements depend, to a large extent, only on the geometric details of the elements. For transverse protrusions or grooves, the dominant features are separation and reattachment, while visual studies have confirmed the existence of flow rotation and separation for spirally-ridged surfaces. From much that has been presented so far, it is quite evident that the geometric parameters of roughness, in determining the prevailing local flow conditions, also produce a global effect on transition, in a manner that is consistent with the well established influence of single and two-dimensional roughness in incompressible boundary layer flows (Schlichting, 1979). Although it is by now conclusive that transition to turbulence is the main physical phenomenon which determines the attainable friction and average heat transfer coefficients, the question as to whether it also determines the complex local heat transfer trends in rough passages may appear at first to be open to conjecture. But, since average heat transfer coefficients are simply the area integrated values of the local coefficients, it is reasonable to expect that transition would play a decisive role on the local trends. Studies with smooth and rough cylinders support this view (Kestin and Maeder, 1957; Achenbach, 1977; 1975).

Another general comment deals with the conventional Reynolds number similarity which, until recently, has been the sole basis for determining whether two or more channel flows are similar. It was demonstrated conclusively that this requirement, which asserts that flows having the same Reynolds number must be similar, is valid only when the critical friction factor and critical Reynolds number for transition are the same, 
but totally breaks down if the latter conditions are not satisfied (Obot, 1988). The connection between transition and flow similarity, which is also covered thoroughly in this paper, should be considered for all flows (boundary layers, cross-flow over tube banks, etc.) that are characterized by transition from laminar to turbulent motion. For rod bundles, the critical Reynolds numbers are significantly lower than the smooth tube values (Rehme, 1973) and the prevailing heat transfer coefficients are also determined by transition. In the case of boundary layers, since the flow development on a wind tunnel model can be markedly different from that on a full scale unit, at least insofar as the onset of transition is concerned, the results in this report make it perfectly clear that application of the law of corresponding states may be central to a proper interpretation of experimental data as well as scale-up.

\section{CONCLUSIONS AND RECOMMENDATIONS}

\subsection{Conclusions}

The determination of the role of transition on friction and convective heat transfer, which was the objective of this study, has been to a considerable extent accomplished satisfactorily. The most important observations are as follows:

1. The onset of transition to turbulence determines the attainable friction and heat transfer coefficients in smooth and rough passages.

2. According to the frictional law of corresponding states, different types of roughness exhibit the same general features at the same reduced conditions. The onset of the fully rough flow regime is almost definite in value and character, beginning at about $R e_{m}=$ $1.3 R e_{m, c} \cdot$

3. For rough passages, the marked increases in frictional pressure coefficient are intimately associated with early transition, and when this role of transition is accounted 
for, the reduced friction factors are considerably lower than the values deduced from the conventional $f$ vs. Re plots.

4. For heat transfer, the simple rule amounts to this: the lower the critical Reynolds number for transition, the greater the average heat transfer coefficients, regardless of whether the passage is smooth or rough. The practical implication of this observation is that definite conclusions on the thermal performance of various surfaces and geometries can be reached based solely on knowledge of the critical Reynolds numbers for transition.

5. For smooth passages, heat transfer coefficients may be greatest with triangular passages, lowest with ducts of annular or rectangular geometry, but intermediate values should be expected for circular tubes.

6. For rough passages, there are clear indications that estimates of the average heat transfer coefficient, that are quite satisfactory for design purposes, can be obtained from the smooth tube data by simply replacing the familiar Reynolds number $(R e)$ with the reduced value $\left(R e_{m}\right)$ computed from $R e_{m}=\psi_{R} R e_{a}$. Furthermore, expressed as the ratio $\phi_{f} / \phi_{h, \max }$, where $\phi_{f}=f_{m} / f_{m, s}$ and $\phi_{h, \max }=\left(N u_{r} / N u_{s}\right)_{\max }$, the condition $\phi_{f} / \phi_{h, \max } \leq 2$ is satisfied by most of the data considered in the analysis.

\subsection{Recommendations}

The law of corresponding states for friction, like all other theories, demands extensive experimental verification and clear statements of the ranges over which it is applicable before it can be used with confidence. For smooth passages, that aspect of the theory which deals with friction has been validated conclusively (Obot, 1988). There is need, however, for detailed heat transfer studies to establish the effects of duct geometry; notably, for triangular passages, the past studies of which have resulted in conflicting conclusions. Given the amount of experimental information in the literature for rough passages, the almost total neglect of the laminar and transition regions by 
researchers, even in the case of pressure drop, is difficult to explain. Studies on pressure drop and heat transfer with various geometries of roughness are needed. Even if heat transfer measurements do not cover the laminar or transition region, it is most desirable to obtain very accurate pressure drop measurements for the laminar, transition and turbulent flow regimes. For such measurements, the following points are worthy of note: first, sufficient taps should be used to obtain truly representative mean pressure drop; second, precise locations of the upstream and downstream taps, relative to the roughness elements, should be noted and reported (Obot et al., 1987); third, it is most desirable to obtain data with and without heat transfer.

With regard to the phenomenon of transition, experimental and theoretical studies are needed to improve the current state of knowledge on the subject. Specifically, there is the need to establish simple and direct experimental methods for accurate determination of the critical parameters at the onset of transition. The prediction of transition has received very meager treatment in the past. This unfortunate situation must be corrected to provide better understanding of the transition process, turbulence and the attendant heat/mass transfer.

\section{ACKNOWLEDGEMENTS}

The work by N. T. Obot and E. B. Esen was performed as part of the Thermal Sciences Research Program sponsored by the U. S. Department of Energy, Office of Energy Utilization Reasearch, under the Energy Conversion and Utilization Technologies (ECUT) Program, Contract No. DE-FG02-89CE90029.A000. The continuing encouragement and support of the Program Managers, D. H. Knoebel and M. E. Gunn, are gratefully appreciated. Technical support was provided by Mr. A. Thomas, Manager, Experimental Systems Engineering, ES Division, Argonne National Laboratory. The 
work by T. J. Rabas was partially funded under Contract W-31-109-Eng-38. 


\section{REFERENCES}

Achenbach, E., 1977, "The Effect of Surface Roughness on the Heat Transfer from a Circular Cylinder to the Cross Flow of Air," Int. J. Heat Mass Transfer, Vol. 20, pp. 359-369.

Achenbach, E., 1975, "Total and Local Heat Transfer from a Smooth Circular Cylinder in Crossflow at High Reynolds Number," Int. J. Heat Mass Transfer, Vol. 18, pp. 1387-1396.

Bandopadhayay, P. C., and Hinwood, J. B., 1973, “On the Coexistence of Laminar and Turbulent Flow in a Narrow Triangular Duct," J. Fluid Mech., Vol. 59, pp. 775-783.

Blackwelder, R., and Kreith, F., 1970, "An Experimental Investigation of Heat Transfer and Pressure Drop in a Decaying Swirling Flow," in Augmentation of Convective Heat and Mass Transfer, ASME Heat Transfer Div., A. E. Bergles and R. L. Webb, Eds., American Society of Mechanical Engineers, New York, NY.

Campbell, D. A., and Perkins, H. C., 1968, "Variable Property Turbulent Heat and Momentum Transfer for Air in a Vertical Rounded Corner Triangular Duct," Int. J. Heat Mass Transfer, Vol. 11, pp. 1003-1012.

Carlson, L. W., 1959, “Experimental Friction Factors for Fully Developed Turbulent Flow in Smooth Triangular Ducts," MS Thesis, University of Minnesota.

Cebeci, T., and Smith, A. M. O., 1968, "Investigation of Heat Transfer and Suction for Tripping Laminar Boundary Layers," J. Aircraft, Vol. 5, p. 450.

Colburn, A. P., 1933, "A Method of Correlating Forced Convection Heat Transfer Data and a Comparison with Fluid Friction," Trans. AIChE, Vol. 29, pp. 174-210.

Dalle Donne, M., and Meyer, L., 1977, "Turbulent Convective Heat Transfer from Rough Surfaces with Two-Dimensional Rectangular Ribs," Int. J. Heat Mass Transfer, Vol. 20, pp. 583-620. 
Dawson, D. A., and Trass, O., 1972, "Mass Transfer at Rough Surfaces," Int J. Heat Mass Transfer, Vol. 15, pp. 1317-1336.

Dipprey, D. F., and Sabersky, R. H., 1963, "Heat and Momentum Transfer in Smooth and Rough Tubes at Various Prandtl Numbers," Int. J. Heat Mass Transfer, Vol. 6, pp. 329-353.

Dodge, W., and Metzner, A. B., 1959, "Turbulent Flow of Non-Newtonian Systems," AIChE J., Vol. 5, pp. 189-204.

Drexel, R. E., and McAdams, W. H., 1944, "Heat-Transfer Coefficients for Air Flowing in Round Tubes, in Rectangular Ducts, and Around Finned Cylinders," National Advisory Committee for Aeronautics, Advance Restriction Report, ARR No. 4F28.

Dryden, H. L., 1953, "Review of Published Data on the Effect of Roughness on Transition from Laminar to Turbulent Flow, Journal of Aeronautical Sciences, Vol. 20, pp. 477-482.

Eckert, E. R. G., and Irvine, T. F., 1960, "Pressure Drop and Heat Transfer in a Duct with Triangular Cross Section," Trans. ASME J. Heat Transfer, Vol. 82, pp. 125-138.

Eckert, E. R. G., and Irvine, T. F., 1956, "Flow in Corners of Passages with Noncircular Cross Sections," Trans. ASME, Vol. 78, pp. 709-718.

Gee, D. L., and Webb, R. L., 1980, "Forced Convective Heat Transfer in Helically Rib-Roughened Tubes," Int. J. Heat Mass Transfer, Vol. 23, pp. 1127-1136.

Gomelauri, V., 1964, "Influence of Two-Dimensional Artificial Roughness on Convective Heat Transfer," Int. J. Heat Mass Transfer, Vol. 7, pp. 653-663.

Han, J. C., Glicksman, L. R., and Rohsenow, W. M., 1978, "An Investigation of Heat Transfer and Friction for Rib-Roughened Surfaces," Int. J. Heat Mass Transfer, Vol. 21, pp. 1143-1156.

Hartnett, J. P., Koh, J. C. Y., and McComas, S. T., 1962, "A Comparison of 
Predicted and Measured Friction Factors for Flow Through Rectangular Ducts," Trans. ASME J. Heat Transfer, Vol. 84, pp. 82-88.

Kestin, J., and Maeder, P. F., 1957, "Influence of Turbulence on the Transfer of Heat from Cylinders," National Advisory Committee for Aeronautics, Technical Note 4018.

Kestin, J., Maeder, P. F., and Wang, H. E., 1961, "Influence of Turbulence on the Transfer of Heat from Plates With and Without a Pressure Gradient," Int. Dev. in Heat Transfer, ASME, pp. 432-438.

Knudsen, J. G., and Katz, D. L., 1950, "Heat Transfer and Pressure Drop in Annuli. Measurements on Plain and Transverse Fin Tubes Using Water," Chem. Eng. Progress, Vol. 46, \# 10, pp. 490-500.

Koch, R., 1960, "Pressure Loss and Heat Transfer for Turbulent Flow," United States Atomic Energy Commission, AEC-tr-3875.

Koch, R., and Feind, K., 1958, "Druckverlust und Wärmeübergang in Ringspalten," Chemie-Ing.-Techn., Vol.30, pp. 577-584.

Lanchester, F. W., 1913, "Surface Cooling and Skin Friction," Reports and Memoranda, No. 94, Brit. Adv. Comm. Aero., Technical Report 1912-1913, pp. 40-44.

Li, H. M., Ye, K. S., Tan, Y. K., and Deng, S. J., 1982, "Investigation of Tube-Side Flow Visualization, Friction Factors and Heat Transfer Characteristics of Helical-Ridging Tubes," Proc. 7th Int. Heat Transfer Conf., Vol. 3, pp. 75-80.

Liu, K. V., Choi, U. S., and Kasza, K. E., 1988, "Measurements of Pressure Drop and Heat Transfer in Turbulent Pipe Flows of Particulate Slurries," Argonne National Laboratory Report ANL-88-15.

Marner, W. J., and Bergles, A. E., 1978, “Augmentation of Tubeside Laminar Flow Heat Transfer by Means of Twisted-Tape, Static-Mixer Inserts, and Internally Finned Tubes," Proc. 6th Int. Heat Transfer Conf., Vol. 2, pp. 583-588. 
Matthys, E. F., Ahn, H., and Sabersky, R. H., 1987, "Friction and Heat Transfer Measurements for Clay Suspensions with Polymer Additives," Trans. ASME J. Fluid Eng., Vol. 109, pp. 307-312.

McAdams, W. H., 1942, Heat Transmission, Second Edition, McGraw-Hill Book Company, Inc., New York, pp. 154-207.

Molloy, J,. 1967, "Rough Tube Friction Factors and Heat Transfer Coefficients in Laminar and Transition Flow,"Atomic Energy Research Establishment (U. K.), AERER-5415.

Morkovin, M. V., 1958, "Transition From Laminar to Turbulent Shear Flow-A Review of Some Recent Advances in Its Understanding," Trans. ASME, Vol. 80, pp. 1121-1128.

Nikuradse, J., 1933, "Laws of Flow in Rough Pipes," National Advisory Committee for Aeronautics Technical Memorandum 1292.

Nunner, W., 1956, "Heat Transfer and Pressure Drop in Rough Tubes," Atomic Energy Research Establishment (U. K.), Lib/Trans. 786.

Obot, N. T., 1988, "Determination of Incompressible Flow Friction in Smooth Circular and Noncircular Passages: A Generalized Approach Including Validation of the Century Old Hydraulic Diameter Concept," Trans. ASME J. Fluids Eng., Vol. 110, pp. 431-440.

Obot, N. T., Esen, E. B., and Adu-Wusu, K., 1987, "Pressure Drop For RibRoughened Scalene Triangular Duct Having Two Rounded Corners," Int. Comm. Heat Mass Transfer, Vol. 14, pp. 11-20.

Owens, P. R., and Thomson, W. R., 1963, "Heat Transfer Across Rough Surfaces," J. Fluid Mech., Vol. 15, pp. 321-334.

Petukhov, B. S., and Popov, V. N., 1963, "Theoretical Calculation of Heat Exchange and Frictional Resistance in Turbulent Flow in Tubes of an Incompressible Fluid 
with Variable Physical Properties," High Temperature, Vol. 1, pp. 69-83.

Petukhov, B. S., and Roizen, L. I., 1963, "An Experimental Investigation of Heat Transfer in a Turbulent Flow of Gas in Tubes of Annular Section," High Temperature, Vol. 1, pp. 373-380.

Prandtl, L., 1928, Physik. Z., pp. 487-489.

Rabas, T. J., 1989, "Selection of the Energy-Efficient Enhancement Geometry For Single-Phase Turbulent Flow Inside Tubes," maunscript submitted to the 26th ASME/AIChE National Heat Transfer Conference, Philadelphia, Pennslyvania, Aug. 6-9.

Ravigururajan, T. S., and Bergles, A. E., 1986, "Study of Water-Side Enhancement for Ocean Thermal Conversion Heat Exchangers," HTL-44/ERI Project 1718, Iowa State University.

Rehme, K., 1973, "Simple Method of Predicting Friction Factors of Turbulent Flow in Non-Circular Channels," Int. J. Heat Mass Transfer, Vol. 16, pp. 933-950.

Reynolds, O., 1883, "An Experimental Investigation of the Circumstances which Determine Whether the Motion of Water Shall be Direct or Sinuous, and of the Law of Resistance in Parallel Channels," Phil. Trans. Roy. Soc. London, Vol. 174, Series A, pp. 935-982.

Reynolds, O., 1874, "On the Extent and Action of the Heating Surface of Steam Boilers,", in Scientific Papers, Vol. 1, Cambridge Unversity Press, Cambridge, pp. 8185.

Savage, D. W., and Myers, J. E., 1963, "The Effect of Artificial Surface Roughness on Heat and Momentum Transfer," AIChE J., Vol. 9, \# 5, pp. 694-702.

Schlichting, H., 1979, Boundary Layer Theory, Seventh Edition, McGraw-Hill Book Company, New York.

Sieder, E. N., and Tate, G. E., 1936, "Heat Transfer and Pressure Drop of Liquids 
in Tubes," Ind. Eng. Chem., Vol. 28, pp. 1429-1435.

Smith, J. W., and Gowen, R. A., 1965, "Heat Transfer Efficiency in Rough Pipes at High Prandtl Number," AIChE J, Vol. 11, \# 5, pp. 941-943.

Stanton, T. E., 1897, "On the Passage of Heat Between Metal Surfaces and Liquids in Contact with Them," Phil. Trans. Roy. Soc. London, Vol. 190, Series A, pp. 67-88.

Stanton, T. E., 1913, "Note on the Relation between Skin Friction and Surface Cooling," Brit. Adv. Comm. Aero., Technical Report 1912-1913, pp. 45-47.

Taylor, G. I., 1916, "Conditions at the Surface of a Hot Body Exposed to the Wind," Brit. Adv. Comm. Aero., Reports and Memoranda, No. 272.

Tokarev, Yu. I., 1979, "Experimental Investigation of Heat Transfer in a Channel Triangular in Cross Section," Thermal Eng., Vol. 26, \# 10, pp. 605-606.

Washington, L., and Marks, W. M., 1937, "Heat Transfer and Pressure Drop in Rectangular Air Passages," Ind. Eng. Chem., Vol. 29, pp. 337-345.

Watkinson, A. P., Miletti, D. L., and Kubanek, G. R., 1974, "Heat Transfer and Pressure Drop of Forge-Fin Tubes in Laminar Oil Flow," Noranda Research Center, Internal Report \# 303.

Watkinson, A. P., Miletti, D. L., and Tarassof, P., 1973, “Turbulent Heat Transfer and Pressure Drop in Internally Finned Tubes," AIChE Symp. Series, Vol. 69, \# 131, pp. 94-103.

Wazzan, A. R., Okamura, T., and Smith, A. M. O., 1968, "The Stability of Water Flow over Heated and Cooled Plates," Trans. ASME J. Heat Transfer, Vol. 90, pp. 109-114.

Webb, R. L., 1987, "Enhancement of Single-Phase Heat Transfer," in Handbook of Single-Phase Convective Heat Transfer, S. Kakac, R. K. Shah, and W. Aung, Eds., John Wiley and Sons, New York, NY.

Webb, R. L., Eckert, E. R. G., and Goldstein, R. J., 1971, "Heat Transfer and 
Friction in Tubes with Repeated-Rib Roughness," Int. J. Heat Mass Transfer, Vol. 14, pp. 601-617.

Withers, J. G., 1980a, "Tube-Side Heat Transfer and Pressure Drop for Tubes Having Helical Internal Ridging with Turbulent/Transitional Flow of Single-Phase Fluid. Part 1. Single-Helix Ridging," Heat Transfer Eng., Vol. 2, \# 1, pp. 48-58.

Withers, J. G., 1980b, “Tube-Side Heat Transfer and Pressure Drop for Tubes Having Helical Internal Ridging with Turbulent/Transitional Flow of Single-Phase Fluid. Part 2. Multiple-Helix Ridging," Heat Transfer Eng., Vol. 2, \# 2, pp. 43-50. 


\section{APPENDIX}

\section{TABULATION OF DATA}

This appendix gives a complete tabulation of the data used in the preparation of the illustrations presented in Sections 2.1 through 5. There are twelve tables, A.1 through A.12, each of which gives the data for a particular study. Several points are worthy of note. First, for smooth or enhanced tubes, $f_{m}$ or $\mathrm{Re}_{\mathrm{m}}$ are independent of the length scale used to reduce the original data to nondimensional form. For the heat transfer data, $\mathrm{Nu}_{m}$ is the Nusselt number for a particular value of $R e_{m}\left(=\downarrow_{R} R e\right)$. Since it is an unscaled parameter, it has the same numerical value as the corventional Nusselt number (Nu). To emphasize this important point, both $\mathrm{Nu}$ and $\mathrm{Nu}_{\mathrm{m}}$ are given in the appropriate tables.

Because of the large amount of information, the use of additional coding to identify some of the data sets was unavoidable. The following comments will be helpful to the reader.

1. For the heat transfer data of Koch and Feind (1958), Table A.6(b), the columns labeled $\mathrm{Re}_{\mathrm{m}}$ (9) and $\mathrm{Re}_{\mathrm{m}}(10)$ correspond to the data for Figs. 9 and 10 respectively. It should be noted that the original (conventiona) Reynolds numbers were scaled with the rounded reference value $\left(R e_{c,}\right)$ of 2,100 for Fig. 9, while the $\mathrm{Re}_{\mathrm{c}, \mathrm{r}}$ value (2719) of the original data was used for Fig. 10.

2. For Table A.7, which gives Koch's friction and heat transfer data, some of the data blocks are assigned letters (e.g., (e), (f), (g), etc.) in addition to the geometric parameters of the roughness. These correspond to letters in the original publication. Also, in the summary of the Stanton number ratios, the values for the friction Reynolds number $\left(R e_{\mathrm{tm}}\right)$ are not given because these can be easily calculated from $R e_{c m}=R e_{m}\left(t_{m} / 2\right)^{1 / 2}$. And, further, there are differences in $\rangle_{t}$ or $t_{R}$ values betwen friction and heat transfer data. For all friction data, the reference $f_{c}$ and $R e_{c, t}$ values were 0.008 and 2,100 (Figs. 6 and 7 ). For heat transfer, it was more appropriate, as demonstrated from comparison between Figs. 9 and 10, to use the actual $f_{c,}$ and $R \theta_{c, r}$ values determined from the original papers.

3. For Table A.8, which gives Nunner's data, the blocks are also identified using the original labeling of the author (1.e., A20, B80, etc.), the description of the elements as well as our in-house identification numbers (i.e., (1), (2), (3), etc.). For friction, $\Psi_{f}$ and $\psi_{R}$ were determined using $f_{c, r}=0.008$ 
and $R e_{G,}=2,100$; the same approach as with Koch's data. As noted already in Section 3.2, for heat transfer calculations, $\downarrow_{\mathrm{A}}$ values were deduced from heat transfer deta; hence there are significant differences between the values for friction and heat transfer. 
Table A.1

Allen and Grunberg (1937)

Smooth rectangular duct friction factor data

$a_{1}=3.92, \downarrow_{A}=0.907, \downarrow_{1}=1.0$

\begin{tabular}{|c|c|c|c|}
\hline Re & $f$ & $\mathbf{R} \boldsymbol{\theta}_{m}$ & $f_{m}$ \\
\hline 493 & 0.0332 & 544 & 0.0332 \\
\hline 630 & 0.0262 & 695 & 0.0262 \\
\hline 778 & 0.0202 & 858 & 0.0202 \\
\hline 907 & 0.0180 & 1000 & 0.018 \\
\hline 1096 & 0.0146 & 1208 & 0.0146 \\
\hline 1291 & 0.0124 & 1423 & 0.0124 \\
\hline 1385 & 0.0115 & 1527 & 0.0115 \\
\hline 1575 & 0.0104 & 1736 & 0.0104 \\
\hline 1879 & 0.00827 & 2072 & 0.00827 \\
\hline 2016 & 0.00789 & 2223 & 0.00789 \\
\hline 3715 & 0.0092 & 4096 & 0.009 \\
\hline 4132 & 0.0092 & 4556 & 0.0092 \\
\hline 4877 & 0.0089 & 5377 & 0.008 \\
\hline 5964 & 0.00894 & 6576 & 0.00894 \\
\hline 10650 & 0.0076 & 11742 & 0.0076 \\
\hline 13655 & 0.00727 & 15055 & 0.00727 \\
\hline 15010 & 0.00727 & 16549 & 0.00727 \\
\hline 16115 & 0.00692 & 17767 & 0.00692 \\
\hline
\end{tabular}


Table A.2

Comish (1928)

Smooth rectangular duct friction factor data

Series 1: $a_{1}=2.92, \downarrow_{A}=0.944, t_{t}=1.0$

\begin{tabular}{|c|c|c|c|c|c|c|c|}
\hline Ro & 1 & $\mathbf{R} \boldsymbol{o}_{m}$ & $t_{n}$ & Ro & $f$ & $\boldsymbol{n}_{\mathrm{m}}$ & $t_{m}$ \\
\hline 270 & 0.06320 & 205 & 0.063800 & 3716 & 0.00046 & 3507 & 0.00046 \\
\hline 282 & 0.05760 & 288 & 0.05760 & 3006 & 0.00946 & 3771 & 0.00046 \\
\hline 206 & 0.05740 & 278 & 0.05740 & 4020 & 0.00044 & 3784 & 0.00044 \\
\hline 313 & 0.05320 & 205 & 0.05820 & 4000 & 0.00882 & 4625 & 0.00892 \\
\hline 871 & 0.04400 & 300 & 0.04400 & 6568 & 0.00836 & 6199 & 0.00836 \\
\hline 419 & 0.04460 & 305 & 0.04160 & 7780 & 0.00802 & 7343 & 0.00802 \\
\hline 445 & 0.03820 & 420 & 0.03820 & 8000 & 0.00782 & 8117 & 0.00782 \\
\hline 553 & 0.03020 & 522 & 0.03020 & 10000 & 0.00756 & 9438 & 0.00756 \\
\hline 584 & 0.02004 & 551 & 0.00804 & 13720 & 0.00710 & 12949 & 0.00710 \\
\hline 796 & 0.02068 & 751 & 0.02058 & 20200 & 0.00548 & 19065 & 0.00648 \\
\hline 804 & 0.02072 & 750 & 0.02072 & 26120 & 0.00604 & 24652 & 0.00604 \\
\hline 1372 & 0.01264 & 1205 & 0.01264 & 30320 & 0.00574 & 31447 & 0.00574 \\
\hline 1400 & 0.01244 & 1321 & 0.01244 & 58509 & 0.00570 & 31711 & 0.00570 \\
\hline 1861 & 0.00972 & 1756 & 0.00072 & 34320 & 0.00566 & 32391 & 0.00566 \\
\hline 2004 & 0.00944 & 1891 & 0.00944 & 41480 & 0.00542 & 39149 & 0.00542 \\
\hline 2009 & 0.00898 & 1881 & 0.00898 & 42080 & 0.00532 & 39715 & 0.00532 \\
\hline 2224 & 0.00864 & 2009 & 0.00864 & 42280 & 0.00534 & 39904 & 0.00534 \\
\hline 2484 & 0.00874 & 2344 & 0.00874 & 42960 & 0.00538 & 40546 & 0.00538 \\
\hline 2510 & 0.00500 & 2397 & 0.00000 & 53280 & 0.00512 & 50286 & 0.00512 \\
\hline 2544 & 0.00904 & 2401 & 0.00504 & 55040 & 0.00512 & 51947 & 0.00512 \\
\hline 2952 & 0.00956 & 2767. & 0.00956 & 56160 & 0.00508 & 53004 & 0.00506 \\
\hline 3152 & 0.00074 & 2075 & 0.00074 & 65240 & 0.00498 & 59686 & 0.00492 \\
\hline 3268 & $0.00 s e 2$ & 3084 & 0.00982 & 63720 & 0.00494 & 60139 & 0.00494 \\
\hline
\end{tabular}


Sories 2: $a_{1}=2.92, \nabla_{R}=0.944, t_{t}=1.0$

\begin{tabular}{|c|c|c|c|c|c|c|c|}
\hline Po & 1 & $\mathbf{A o}_{\mathrm{m}}$ & $f_{m}$ & $\mathbf{A D}_{0}$ & 1 & $\mathbf{P}_{\mathbf{o}_{m}}$ & $f_{m}$ \\
\hline 350 & 0.047 & 330 & 0.047 & 2636 & 0.00036 & 2468 & 0.00936 \\
\hline 368 & 0.0452 & 345 & 0.0452 & 2636 & 0.00014 & 2488 & 0.00014 \\
\hline 610 & 0.0278 & 584 & 0.0278 & 2651 & 0.00916 & 2500 & 0.00016 \\
\hline 963 & 0.0176 & $\infty 00$ & 0.0176 & 2828 & 0.0008 & 2060 & 0.0008 \\
\hline 1004 & 0.0166 & 248 & 0.0168 & 3524 & 0.00078 & 3326 & 0.00978 \\
\hline 1406 & 0.0116 & 1412 & 0.0116 & 5000 & 0.00604 & 4718 & 0.00084 \\
\hline 1648 & 0.0105 & 1556 & 0.0105 & 5040 & 0.00888 & 4757 & 0.00888 \\
\hline 1751 & 0.00006 & 1653 & 0.00086 & 5056 & 0.00000 & 4772 & 0.00000 \\
\hline 1964 & 0.0080 & 1854 & 0.0089 & 7156 & 0.00826 & 6754 & 0.00826 \\
\hline 2140 & 0.00824 & 2000 & 0.00824 & 8384 & 0.00802 & 7913 & 0.00802 \\
\hline 2143 & 0.0082 & 20023 & 0.0082 & 8960 & 0.00792 & 8456 & 0.00792 \\
\hline 2176 & 0.00826 & 2054 & 0.00826 & 9320 & 0.00782 & 8796 & 0.00782 \\
\hline 2098 & 0.00022 & 2258 & 0.00822 & 10480 & 0.00756 & $\infty 091$ & 0.00756 \\
\hline 2400 & 0.00814 & 2265 & 0.00814 & 11860 & 0.00738 & 11268 & 0.00738 \\
\hline 2416 & 0.00816 & 2230 & 0.00816 & 12400 & 0.00728 & 11703 & 0.00728 \\
\hline 2416 & 0.00792 & 2280 & 0.00782 & 12760 & 0.00726 & 12043 & 0.00726 \\
\hline 2540 & 0.00874 & 2397 & 0.00874 & 20200 & 0.00652 & 18065 & 0.00652 \\
\hline
\end{tabular}




\section{Table A.3}

\section{Cartson (1959)}

Smooth triangular duct friction factor data

Duct $1: h / b=14.26, \alpha=4.01^{\circ}, \nabla_{A}=1.512, \nabla_{1}=1.0$

\begin{tabular}{|c|c|c|c|c|c|c|c|}
\hline Po & 1 & $R_{0}$ & $t_{n}$ & no & 1 & $\mathbf{R o}_{\mathrm{m}}$ & $i_{m}$ \\
\hline 468 & 0.00510 & 706 & 0.002540 & 2170 & 0.00043 & 3282 & 0.00043 \\
\hline 575 & 0.02140 & 870 & 0.02140 & 2410 & 0.00918 & 3544 & 0.00018 \\
\hline 682 & 0.01820 & 1031 & 0.01820 & 2540 & 0.00003 & 3841 & 0.00003 \\
\hline 704 & 0.01550 & 1201 & 0.01550 & 3140 & 0.00848 & 4748 & 0.00848 \\
\hline$\infty 00$ & 0.01400 & 1331 & 0.01400 & 3700 & 0.00000 & 5688 & 0.00803 \\
\hline 1140 & 0.01000 & 1724 & 0.01090 & 4250 & 0.00788 & 6427 & 0.00788 \\
\hline 1387 & 0.00883 & 2097 & 0.00883 & 4710 & 0.00768 & 7122 & 0.00768 \\
\hline 1637 & 0.01040 & 2476 & 0.01040 & 5330 & 0.00740 & 8060 & 0.00740 \\
\hline 1805 & 0.00995 & 2866 & 0.00995 & 5880 & 0.00740 & 8043 & 0.00740 \\
\hline
\end{tabular}

Duct 2: $h / b=7.21, \alpha=7.96^{\circ}, \downarrow_{R}=1.302, \downarrow_{1}=1.0$

\begin{tabular}{|c|c|c|c|c|c|c|c|}
\hline Ro & 1 & $\mathbf{P o}_{\mathrm{m}}$ & $f_{m}$ & Re & 1 & $\mathbf{R}_{\mathrm{m}}$ & $f_{m}$ \\
\hline 563 & 0.02180 & 733 & 0.02190 & 3035 & 0.00880 & 3052 & 0.00880 \\
\hline 664 & 0.01920 & 865 & 0.01920 & 3200 & 0.00840 & 4713 & 0.00840 \\
\hline$\pi 2$ & 0.01630 & 1005 & 0.01630 & 4259 & 0.00800 & 5545 & 0.00800 \\
\hline 879 & 0.01470 & 1145 & 0.01470 & 4920 & 0.00780 & 6406 & 0.00780 \\
\hline 1116 & 0.01140 & 1453 & 0.01140 & 6570 & 0.00753 & 7252 & 0.00753 \\
\hline 1350 & 0.00035 & 1769 & 0.00035 & 6250 & 0.00740 & 8138 & 0.00740 \\
\hline 1615 & 0.00820 & 2103 & 0.00820 & 6880 & 0.00713 & 9088 & 0.00713 \\
\hline 1809 & 0.01000 & 2434 & 0.01000 & 7760 & 0.00700 & 10104 & 0.00700 \\
\hline 2130 & 0.00060 & 2773 & 0.00900 & 8600 & 0.00580 & 11197 & 0.00880 \\
\hline 2380 & 0.00950 & 3009 & 0.00930 & 9700 & 0.00680 & 12020 & 0.00650 \\
\hline 2580 & 0.00930 & 3350 & 0.00950 & 10650 & 0.00650 & 13866 & 0.00650 \\
\hline
\end{tabular}


Duct 3: $h / B=4.75, a=120^{\circ}, \downarrow_{A}=1.356, \downarrow_{f}=1.0$

\begin{tabular}{|c|c|c|c|c|c|c|c|}
\hline A & 1 & $\mathbf{n}_{m}$ & $t_{m}$ & Po & 1 & $\mathbf{R o}_{m}$ & $i_{m}$ \\
\hline 440 & 0.00840 & 67 & 0.02040 & 16800 & 0.00508 & 22510 & 0.00598 \\
\hline 546 & 0.00200 & 740 & 0.002000 & 24200 & 0.00548 & 32815 & 0.00548 \\
\hline 643 & 0.02000 & 872 & 0.02000 & 2420 & 0.00080 & 3282 & 0.00080 \\
\hline 748 & 0.01700 & 1014 & 0.01700 & 8020 & 0.00003 & 4005 & 0.00003 \\
\hline 857 & 0.01480 & 1182 & 0.01480 & 8680 & 0.00863 & 4003 & 0.00863 \\
\hline 1084 & 0.0120 & 1470 & 0.01220 & 4000 & 0.00848 & 5546 & 0.00848 \\
\hline 1315 & 0.00080 & 1780 & 0.00080 & 4510 & 0.00833 & 6116 & 0.00833 \\
\hline 1580 & 0.00848 & 2115 & 0.00348 & $\infty 000$ & 0.00780 & 8136 & 0.00780 \\
\hline 1810 & 0.01040 & 2454 & 0.01040 & 7280 & 0.00723 & 8872 & 0.00723 \\
\hline 2058 & 0.01020 & 2790 & 0.01020 & 8710 & 0.00070 & 11811 & 0.00070 \\
\hline 2310 & 0.00988 & 3132 & 0.00988 & 9800 & 0.00685 & 13533 & 0.00685 \\
\hline 2430 & 0.00930 & 3000 & 0.00030 & 11500 & 0.00650 & 15594 & 0.00650 \\
\hline 4130 & 0.00835 & 5600 & 0.00835 & 12820 & 0.00640 & 17384 & 0.00640 \\
\hline 7340 & 0.00715 & 2053 & 0.00715 & 16440 & 0.00600 & 22293 & 0.00600 \\
\hline 11700 & 0.00640 & 15865 & 0.00640 & & & & \\
\hline
\end{tabular}

Duct 4: $h / b=2.54, \alpha=22.3^{\circ}, \downarrow_{R}=1.512, \downarrow_{1}=1.0$

\begin{tabular}{|c|c|c|c|c|c|c|c|}
\hline Ro & $f$ & $\mathbf{R}_{\mathrm{m}}$ & $t_{m}$ & Re & 1 & $\mathbf{P o}_{\mathrm{m}}$ & $f_{m}$ \\
\hline 515 & 0.00500 & 665 & 0.02500 & 2260 & 0.00970 & 2918 & 0.00970 \\
\hline 600 & 0.02070 & 786 & 0.02070 & 2810 & 0.00930 & 3028 & 0.00930 \\
\hline 706 & 0.01790 & 912 & 0.01790 & 3390 & 0.00880 & 4377 & 0.00880 \\
\hline 806 & 0.01620 & 1041 & 0.01620 & 3840 & 0.00883 & 4958 & 0.00883 \\
\hline 1020 & 0.01290 & 1317 & 0.01280 & 4275 & 0.00875 & 5519 & 0.00875 \\
\hline 1242 & 0.01000 & 1604 & 0.01000 & 5020 & 0.00835 & 7256 & 0.00835 \\
\hline 1470 & 0.00915 & 1808 & 0.00915 & 6815 & 0.00805 & 8790 & 0.00805 \\
\hline 1703 & 0.00820 & 2180 & 0.00820 & 8180 & 0.00748 & 10574 & 0.00748 \\
\hline 1703 & 0.00045 & 2180 & 0.00945 & 9400 & 0.00728 & 12136 & 0.00728 \\
\hline 2220 & 0.00980 & 2066 & 0.00880 & 10000 & 0.00050 & 13944 & 0.00690 \\
\hline 2700 & 0.00870 & 3563 & 0.00870 & 12070 & 0.00680 & 15584 & 0.00680 \\
\hline 3580 & 0.00880 & 4622 & 0.00880 & 15630 & 0.00028 & 20180 & 0.00628 \\
\hline 6720 & 0.00788 & 8676 & 0.00788 & 19400 & 0.00605 & 25047 & 0.00605 \\
\hline
\end{tabular}




\begin{tabular}{|c|c|c|c|c|c|c|c|}
\hline Po & 1 & $\boldsymbol{A n}_{m}$ & $t_{m}$ & Po & 1 & $\boldsymbol{R}_{m}$ & $t_{m}$ \\
\hline 8070 & 0.00750 & 10410 & 0.00750 & 20250 & 0.00078 & & \\
\hline 8250 & 0.00735 & 11943 & 0.00735 & 19000 & 0.00808 & 24918 & 0.00608 \\
\hline 11900 & 0.00873 & 15584 & 0.00873 & 25200 & 0.00580 & 29053 & 0.00580 \\
\hline 15420 & 0.00825 & 19000 & 0.00025 & 20200 & 0.00583 & 35827 & 0.00563 \\
\hline 19000 & 0.00005 & 24000 & 0.00006 & 20100 & 0.00548 & $\mathbf{3 7 5 7 1}$ & 0.00548 \\
\hline 22000 & 0.00503 & 28568 & 0.005893 & 31600 & 0.00540 & 40789 & 0.00540 \\
\hline 12050 & 0.00683 & 15552 & 0.00683 & 34100 & 0.00530 & 44027 & 0.00530 \\
\hline 15580 & 0.00533 & 20090 & 0.00333 & & & & \\
\hline
\end{tabular}

Duct 5: $h / b=1.42, a=38.8^{\circ}, \downarrow_{A}=1.512, \downarrow_{f}=1.0$

\begin{tabular}{|c|c|c|c|c|c|c|c|}
\hline Ro & 1 & $\mathbf{P o}_{\mathbf{m}}$ & $f_{m}$ & Re & 1 & $\mathbf{R}_{\mathrm{m}}$ & $t_{m}$ \\
\hline 535 & 0.02500 & 640 & 0.02500 & 8250 & 0.00790 & 8875 & 0.00790 \\
\hline 623 & 0.02130 & 746 & 0.02130 & 7000 & 0.00780 & 8097 & 0.00780 \\
\hline 712 & 0.01800 & 852 & 0.01890 & 8900 & 0.00760 & 10653 & 0.00760 \\
\hline 800 & 0.01470 & $10 \pi$ & 0.01470 & 10500 & 0.00740 & 12569 & 0.00740 \\
\hline 1000 & 0.01200 & 1305 & 0.01260 & 12560 & 0.00710 & 15034 & 0.00710 \\
\hline 1304 & 0.00988 & 1561 & 0.00988 & 14800 & 0.00670 & 17716 & 0.00670 \\
\hline 1500 & 0.00010 & 1796 & 0.00910 & 18150 & 0.00640 & 21726 & 0.00640 \\
\hline 1770 & 0.00785 & 2119 & 0.00785 & 21800 & 0.00620 & 26095 & 0.00020 \\
\hline 1923 & 0.00870 & 2002 & 0.00870 & 26800 & 0.00580 & 32080 & 0.00580 \\
\hline 1990 & 0.01020 & 2082 & 0.01020 & 32650 & 0.00560 & 30082 & 0.00560 \\
\hline 2470 & 0.00990 & 2057 & 0.00990 & 30025 & 0.00545 & 37017 & 0.00545 \\
\hline 2980 & 0.00050 & 3567 & 0.00050 & 34500 & 0.00548 & 41207 & 0.00548 \\
\hline 3350 & 0.00045 & 4010 & 0.00945 & 40900 & 0.00520 & 48057 & 0.00520 \\
\hline 3770 & 0.00828 & 4613 & 0.00928 & 47500 & 0.00500 & 56858 & 0.00500 \\
\hline 4950 & 0.00875 & 5025 & 0.00875 & 57200 & 0.00480 & 68468 & 0.00480 \\
\hline 5000 & 0.00885 & 5085 & 0.00885 & 64000 & 0.00473 & 76608 & 0.00473 \\
\hline 7350 & 0.00818 & 8783 & 0.00818 & & & & \\
\hline
\end{tabular}


Table A.4

Hartnett of al. (1962)

Smooth rectangular duct friction factor data

(a) Abrupt entrance

$a_{1}=1, \downarrow_{\mathrm{A}}=0.978, \downarrow_{1}=1.0$

\begin{tabular}{|c|c|c|c|c|c|c|c|}
\hline Ro & 1 & $\mathbf{R}_{m}$ & $I_{m}$ & Ro & 1 & $\mathbf{R}_{\mathrm{m}}$ & $I_{m}$ \\
\hline 1190 & 0.01000 & 1173 & 0.01000 & 16722 & 0.00660 & 16356 & 0.00650 \\
\hline 1742 & 0.00832 & 1704 & 0.00832 & 20002 & 0.00583 & 26313 & 0.00596 \\
\hline 2585 & 0.00887 & 2528 & 0.00887 & 41862 & 0.00550 & 41034 & 0.00539 \\
\hline 3131 & 0.00920 & 3002 & 0.00020 & 55663 & 0.00497 & 54444 & 0.00497 \\
\hline 4642 & 0.00875 & 4540 & 0.00875 & 75361 & 0.00472 & 73711 & 0.00472 \\
\hline 7094 & 0.00807 & 6039 & 0.00807 & 119002 & 0.00406 & 117297 & 0.00406 \\
\hline 11635 & 0.00715 & 11380 & 0.00715 & 152815 & 0.00390 & 149470 & 0.00390 \\
\hline
\end{tabular}

$a_{1}=5, \downarrow_{A}=0.850, \downarrow_{f}=1.0$

\begin{tabular}{|c|c|c|c|c|c|c|c|}
\hline Ro & $f$ & $\mathbf{P}_{\boldsymbol{\theta}_{m}}$ & $t_{m}$ & Re & $f$ & $\mathbf{R} \boldsymbol{\bullet}_{\mathrm{m}}$ & $f_{m}$ \\
\hline 1020 & 0.01800 & 867 & 0.01800 & 11754 & 0.00767 & 9993 & 0.00767 \\
\hline 1396 & 0.01340 & 1187 & 0.01340 & 15438 & 0.00720 & 13125 & 0.00720 \\
\hline 1657 & 0.01140 & 1409 & 0.01140 & 24320 & 0.00650 & 20677 & 0.00650 \\
\hline 2059 & 0.00875 & 1759 & 0.00875 & 27454 & 0.00820 & 23341 & 0.00020 \\
\hline 2482 & 0.00791 & 2110 & 0.00791 & 30370 & 0.00610 & 25821 & 0.00610 \\
\hline 2001 & 0.00902 & 2288 & 0.009002 & 36425 & 0.00580 & 30000 & 0.00580 \\
\hline 3837 & 0.00968 & 3002 & 0.00968 & 50322 & 0.00539 & 42784 & 0.00539 \\
\hline 4517 & 0.00948 & 3840 & 0.00048 & 56804 & 0.00517 & 48205 & 0.00517 \\
\hline 5458 & 0.00011 & 4630 & 0.00011 & 81711 & 0.00477 & 69471 & 0.00477 \\
\hline 7004 & 0.00857 & 6001 & 0.00857 & 111750 & 0.00427 & 85010 & 0.00427 \\
\hline 9000 & 0.00783 & 8417 & 0.00783 & 198722 & 0.00352 & 168054 & 0.00352 \\
\hline
\end{tabular}


$a_{1}=10, \nabla_{\mathrm{R}}=0.842, t_{1}=1.0$

\begin{tabular}{|c|c|c|c|c|c|c|c|}
\hline Ro & 1 & $\mathbf{P o}_{\mathrm{m}}$ & $t_{m}$ & Po & 1 & $\mathbf{R o}_{m}$ & $t_{m}$ \\
\hline 1042 & 0.02020 & 87 & 0.020020 & 10952 & 0.00007 & $\$ 218$ & 0.00607 \\
\hline 1202 & 0.01600 & 1082 & 0.01600 & 14236 & 0.00744 & 11982 & 0.00744 \\
\hline 1001 & 0.01250 & 1423 & 0.01230 & 22500 & 0.00673 & 18062 & 0.00673 \\
\hline 2175 & 0.00048 & 1831 & 0.00048 & 45032 & 0.0054 & 37903 & 0.00544 \\
\hline 2531 & 0.00840 & 2130 & 0.00840 & 85507 & 0.00440 & 80387 & 0.00449 \\
\hline 5027 & 0.00087 & 2716 & 0.00087 & 145308 & 0.00414 & 122300 & 0.00414 \\
\hline 4736 & 0.00048 & 3906 & 0.00948 & 176046 & 0.00390 & 148175 & 0.00390 \\
\hline 6677 & 0.00884 & 5620 & 0.00884 & & & & \\
\hline
\end{tabular}

(b) Critical Parameters for duct with smooth entrance

\begin{tabular}{|c|c|c|}
\hline$a_{1}$ & $\downarrow_{R}$ & $\downarrow_{f}$ \\
\hline 1 & 0.492 & 2.265 \\
\hline 5 & 0.297 & 2.745 \\
\hline 10 & 0.476 & 1.652 \\
\hline
\end{tabular}


Table A.5

Jones (1976)

Smooth rectangular duct friction factor data

$a_{1}=13, \nabla_{A}=0.811, \downarrow_{1}=1.0$

\begin{tabular}{|c|c|c|c|c|c|c|c|}
\hline no & 1 & $\mathbf{n a m}_{m}$ & in & ino & 1 & $\mathbf{R o}_{\boldsymbol{m}}$ & $t_{m}$ \\
\hline 115 & 0.18000 & 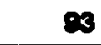 & 0.18000 & 4257 & 0.00558 & 3450 & 0.00058 \\
\hline 144 & 0.13800 & 117 & 0.13800 & 3480 & 0.00016 & 2021 & 0.00016 \\
\hline 165 & 0.12000 & 134 & 0.128000 & roes & 0.00875 & 5005 & 0.00875 \\
\hline 203 & 0.08170 & 200 & 0.08170 & 2000 & 0.00814 & 7475 & 0.00814 \\
\hline 340 & 0.06000 & 278 & 0.00000 & 14055 & 0.00770 & 11394 & 0.00770 \\
\hline 613 & 0.03400 & 497 & 0.03400 & 16470 & 0.00688 & 13352 & 0.00688 \\
\hline 2023 & 0.02170 & 748 & 0.02170 & 18860 & 0.00720 & 15297 & 0.00720 \\
\hline 1037 & 0.01930 & 841 & 0.01930 & 27117 & 0.00658 & 21984 & 0.00658 \\
\hline 1314 & 0.01540 & 1005 & 0.01540 & 27738 & 0.00501 & 22487 & 0.00601 \\
\hline 2212 & 0.00980 & 1793 & 0.00980 & 35500 & 0.00610 & 28853 & 0.00610 \\
\hline 2502 & 0.00836 & 2101 & 0.00836 & 53520 & 0.00570 & 43389 & 0.00570 \\
\hline 3179 & 0.00958 & 2077 & 0.00958 & 78911 & 0.00536 & 62352 & 0.00536 \\
\hline 3460 & 0.00916 & 2821 & 0.00916 & $804 \pi$ & 0.00510 & 65243 & 0.00510 \\
\hline
\end{tabular}

$a_{1}=26, \nabla_{A}=0.724, \downarrow_{f}=1.0$

\begin{tabular}{|c|c|c|c|c|c|c|c|}
\hline Po & 1 & $\mathbf{R o}_{m}$ & $t_{m}$ & Ro & 1 & $\mathbf{R o}_{\mathrm{m}}$ & $t_{m}$ \\
\hline 141 & 0.15000 & 102 & 0.15000 & 4436 & 0.00023 & 3212 & 0.00923 \\
\hline 206 & 0.10200 & 149 & $0.10<00$ & 4778 & 0.01040 & 3459 & 0.01040 \\
\hline 283 & 0.07800 & 205 & 0.07800 & 4967 & 0.00995 & 3596 & 0.00985 \\
\hline 340 & 0.06500 & 246 & 0.06500 & 5598 & 0.00020 & 4053 & 0.00020 \\
\hline 496 & 0.05200 & 316 & 0.05200 & 6413 & 0.00908 & 4643 & 0.00908 \\
\hline 522 & 0.04400 & $\$ 78$ & 0.04400 & 7348 & 0.00887 & 5320 & 0.00887 \\
\hline 717 & 0.05200 & 819 & 0.03200 & 10484 & 0.00836 & 7576 & 0.00838 \\
\hline 880 & 0.02800 & 657 & 0.02600 & 14050 & 0.00790 & 10172 & 0.00790 \\
\hline 1048 & 0.02200 & 757 & 0.02200 & 19738 & 0.00660 & 14290 & 0.00680 \\
\hline 1253 & 0.01860 & 807 & 0.01860 & 24757 & 0.00673 & 17924 & 0.00673 \\
\hline 1403 & 0.01590 & 1016 & 0.01500 & 35579 & 0.00614 & 25759 & 0.00614 \\
\hline
\end{tabular}




\begin{tabular}{|c|c|c|c|c|c|c|c|}
\hline $\mathbf{R o}$ & 1 & $\boldsymbol{P}_{m}$ & 4 & Po & 1 & $\mathbf{P o}_{\mathrm{m}}$ & $t_{m}$ \\
\hline 1681 & 0.01580 & 1217 & 0.01580 & 40504 & 0.00814 & 20245 & 0.00614 \\
\hline 2108 & 0.01130 & 1505 & 0.01130 & 58576 & 0.00547 & 42400 & 0.00547 \\
\hline 2000 & 0.00818 & 2000 & 0.00818 & 76883 & 0.00522 & 56083 & 0.00522 \\
\hline 5780 & 0.00035 & 2743 & 0.00085 & & & & \\
\hline
\end{tabular}

$$
a_{1}=31, \downarrow_{R}=0.757, \downarrow_{t}=1.0
$$

\begin{tabular}{|c|c|c|c|c|c|c|c|}
\hline Ro & 1 & $\mathbf{n}_{\boldsymbol{m}_{m}}$ & $t_{n}$ & P & 1 & $\mathbf{R}_{\mathrm{m}}$ & $t_{m}$ \\
\hline 104 & 021500 & 70 & 0.21500 & 2772 & 0.00840 & 2000 & 0.00840 \\
\hline 151 & 0.16400 & 114 & 0.16400 & 3009 & 0.00020 & 2074 & $0.0002 \geq 0$ \\
\hline 207 & 0.11400 & 157 & 0.11400 & $34 \pi$ & 0.01050 & 2633 & 0.01050 \\
\hline 325 & 0.07500 & 246 & 0.07500 & 4884 & 0.000200 & 3509 & 0.00920 \\
\hline 467 & 0.05000 & 354 & 0.05000 & 7020 & 0.00840 & 5316 & 0.00840 \\
\hline 656 & 0.03400 & 497 & 0.03400 & 10460 & 0.00802 & 7821 & 0.00802 \\
\hline 1046 & 0.00200 & 702 & 0.02200 & 14366 & 0.00730 & 10879 & 0.00730 \\
\hline 1372 & 0.01700 & 1000 & 0.01700 & 23643 & 0.00690 & 17005 & 0.00690 \\
\hline 1645 & 0.01400 & 1246 & 0.01400 & 37214 & 0.00611 & 28182 & 0.00611 \\
\hline 2084 & 0.01100 & 1563 & 0.01100 & 48843 & 0.00560 & 36080 & 0.00560 \\
\hline 2365 & 0.00060 & 1791 & 0.00960 & & & & \\
\hline
\end{tabular}

$$
a_{1}=31, t_{A}=0.811, \downarrow_{1}=1.0
$$

\begin{tabular}{|c|c|c|c|c|c|c|c|}
\hline Re & 1 & $\mathbf{R}_{m}$ & $t_{m}$ & Ro & .1 & $R_{\boldsymbol{o}_{m}}$ & $t_{m}$ \\
\hline 104 & 0.220200 & 84 & 0.22200 & 2500 & 0.00900 & 2100 & 0.00900 \\
\hline 137 & 0.16800 & 111 & 0.16600 & 3478 & 0.00970 & 2820 & 0.00970 \\
\hline 202 & 0.10300 & 164 & 0.10300 & 4463 & 0.00940 & 3610 & 0.00940 \\
\hline 326 & 0.07000 & 284 & 0.07000 & 6561 & 0.00520 & 5020 & 0.00020 \\
\hline 427 & 0.05050 & 346 & 0.05050 & 10509 & 0.00050 & 8407 & 0.00800 \\
\hline 671 & 0.03500 & 54 & 0.005500 & 14371 & 0.00780 & 11652 & 0.00780 \\
\hline 864 & 0.02300 & 782 & 0.02500 & 24202 & 0.00720 & 19023 & 0.00720 \\
\hline 1314 & 0.01770 & 1065 & 0.01770 & 37220 & 0.00650 & 30185 & 0.00550 \\
\hline 1647 & 0.01410 & $13 e 6$ & 0.01410 & 49983 & 0.00590 & 40523 & 0.00590 \\
\hline 1973 & 0.01230 & 1600 & 0.01230 & & & & \\
\hline
\end{tabular}




\section{Table A.6}

Koch and Feind (1958)

Friction and heat transfer data for smooth annuli

(a) Friction factor data

$$
\beta=0.212, \downarrow_{A}=0.731, \psi_{1}=1.0
$$

\begin{tabular}{|c|c|c|c|c|c|c|c|}
\hline Ro & 1 & $\mathbf{P}_{m}$ & in & $\mathbf{n}$ & 1 & $\mathbf{R o}_{\mathrm{m}}$ & $t_{m}$ \\
\hline$\infty \infty$ & 0.05870 & 451 & 0.05870 & 6043 & 0.00000 & 417 & 0.00000 \\
\hline$m e$ & 0.02080 & 879 & 0.02880 & 8355 & 0.00810 & 6107 & 0.00810 \\
\hline 1088 & 0.02160 & ros & 0.02160 & 11024 & 0.00770 & 6058 & 0.00770 \\
\hline 1757 & 0.01350 & 1284 & 0.01350 & 16484 & 0.00716 & 12049 & 0.00716 \\
\hline 2006 & 0.01150 & 1468 & 0.01150 & 18878 & 0.00000 & 13799 & 0.00000 \\
\hline 3014 & 0.00841 & 2203 & 0.00841 & 20780 & 0.00670 & 15189 & 0.00670 \\
\hline 3482 & 0.00000 & 2545 & 0.00500 & 20702 & 0.00635 & 17369 & 0.00665 \\
\hline 4069 & 0.00940 & 2974 & 0.00940 & 32027 & 0.00810 & 23556 & 0.00510 \\
\hline 4642 & 0.00020 & 3903 & 0.00020 & 40079 & 0.00558 & 36532 & 0.00558 \\
\hline 5426 & 0.00905 & 3086 & 0.00005 & & & & \\
\hline
\end{tabular}

$$
\beta=0.409, \downarrow_{A}=0.697, \downarrow_{t}=1.0
$$

\begin{tabular}{|c|c|c|c|c|c|c|c|}
\hline$R_{0}$ & 1 & $\mathbf{R o}_{\mathrm{m}}$ & $t_{m}$ & Ro & 1 & $R_{\bullet_{m}}$ & $t_{m}$ \\
\hline 853 & 0.02070 & 684 & 0.02070 & 6654 & 0.00040 & 4638 & 0.00040 \\
\hline 1187 & 0.01850 & 834 & 0.01950 & 7323 & 0.00860 & 5102 & 0.00860 \\
\hline 1487 & 0.01580 & 1008 & 0.01560 & 11430 & 0.00789 & 7970 & 0.00789 \\
\hline 2130 & 0.01090 & 1484 & 0.01000 & 14776 & 0.00740 & 10205 & 0.00740 \\
\hline 2773 & 0.00850 & 1882 & 0.00850 & 18627 & 0.00700 & 12978 & 0.00700 \\
\hline 3168 & 0.00810 & 2004 & 0.00810 & 22037 & 0.00670 & 15772 & 0.00870 \\
\hline 3525 & 0.00900 & 2456 & 0.00000 & 25260 & 0.00640 & 17600 & 0.00840 \\
\hline 3007 & 0.00050 & 2576 & 0.00050 & 30703 & 0.00610 & 21392 & 0.00610 \\
\hline 4110 & 0.00564 & 2850 & 0.00964 & 38703 & 0.00570 & 20968 & 0.00570 \\
\hline 4756 & 0.00950 & 3314 & 0.00550 & 44256 & 0.00560 & 30835 & 0.00560 \\
\hline 6192 & 0.00940 & 4314 & 0.00940 & 45348 & 0.00545 & 31596 & 0.00545 \\
\hline
\end{tabular}


$\beta=0.6, \downarrow_{A}=0.648, \downarrow_{1}=1.0$

\begin{tabular}{|c|c|c|c|c|c|c|c|}
\hline Ro & 1 & $\mathbf{R}_{\mathrm{m}}$ & 4 & Po & 1 & $\mathbf{P o}_{\mathrm{m}}$ & $t_{m}$ \\
\hline 256 & 0.00040 & 168 & 0.00340 & $\pi \times 0$ & 0.00040 & 5031 & 0.00940 \\
\hline 548 & 0.04800 & 804 & 0.04800 & 12800 & 0.00880 & 8422 & 0.00660 \\
\hline 701 & 0.03100 & 613 & 0.03100 & 16160 & 0.00000 & 10483 & 0.00000 \\
\hline 1575 & 0.01500 & 1021 & 0.01500 & 18237 & 0.00778 & 11858 & 0.00778 \\
\hline 2003 & 0.01130 & 1428 & 0.01130 & 20067 & 0.00770 & 13504 & 0.00770 \\
\hline 2785 & 0.000000 & 1773 & 0.000000 & 23302 & 0.00730 & 15166 & 0.00730 \\
\hline 3157 & 0.00000 & 2047 & 0.00800 & 26483 & 0.00700 & 17170 & 0.00700 \\
\hline$\$ 517$ & 0.00810 & 200 & 0.00840 & 32544 & 0.00658 & 21100 & 0.00658 \\
\hline 3003 & 0.00020 & 2056 & 0.00020 & 42107 & 0.00640 & 27300 & 0.00840 \\
\hline 3918 & 0.00000 & 20540 & 0.00000 & 46271 & 0.00640 & 30000 & 0.00640 \\
\hline
\end{tabular}

$$
\beta=0.698, \downarrow_{R}=0.618, \downarrow_{A}=1.0
$$

\begin{tabular}{|c|c|c|c|c|c|c|c|}
\hline Ro & $f$ & $\mathbf{R}_{\mathrm{m}}$ & $t_{m}$ & Ro & 1 & $\mathrm{Ro}_{\mathrm{m}}$ & $t_{m}$ \\
\hline 302 & 0.07730 & 100 & 0.07730 & 7285 & 0.00000 & 4502 & 0.00000 \\
\hline 398 & 0.06630 & 246 & 0.08630 & 6026 & 0.00860 & 4960 & 0.00860 \\
\hline 506 & 0.04800 & 313 & 0.04800 & 10741 & 0.00810 & 6658 & 0.00810 \\
\hline 545 & 0.04340 & 337 & 0.04340 & 12754 & 0.00780 & 7882 & 0.00780 \\
\hline 738 & 0.03270 & 466 & 0.03270 & 14071 & 0.00770 & 8008 & 0.00770 \\
\hline 796 & 0.03030 & 402 & 0.03030 & 17548 & 0.00740 & 10845 & 0.00740 \\
\hline 822 & 0.02770 & 508 & 0.02770 & 19352 & 0.00710 & 11960 & 0.00710 \\
\hline 1023 & 0.02320 & $\operatorname{cs2}$ & 0.02320 & 21886 & 0.00050 & 13526 & 0.00690 \\
\hline 1544 & 0.01490 & 854 & 0.01490 & 24144 & 0.00670 & 14921 & 0.00670 \\
\hline 2275 & 0.01050 & 1408 & 0.01050 & 26634 & 0.00570 & 16460 & 0.00570 \\
\hline 2850 & 0.00860 & 1740 & 0.00860 & 20744 & 0.00650 & 18382 & 0.00650 \\
\hline 3157 & 0.00780 & 1851 & 0.00780 & 32813 & 0.00630 & 20079 & 0.00630 \\
\hline 3563 & 0.00700 & 2002 & 0.00700 & 37558 & 0.00610 & 23210 & 0.00610 \\
\hline 4171 & 0.00780 & 2058 & 0.00780 & 41841 & 0.00600 & 25020 & 0.00600 \\
\hline 4595 & 0.00050 & 2240 & 0.00950 & 41841 & 0.00000 & 25020 & 0.00600 \\
\hline
\end{tabular}


$\beta=0.793, t_{A}=0.692, t_{1}=1.0$

\begin{tabular}{|c|c|c|c|c|c|c|c|}
\hline As & 1 & $\boldsymbol{m}_{m}$ & $t_{m}$ & An & 1 & $P_{p_{m}}$ & $t_{m}$ \\
\hline 270 & 0.00600 & 187 & 0.00680 & 50037 & 0.00770 & 2100 & 0.00770 \\
\hline 250 & 0.08480 & 248 & 0.06480 & 3342 & 0.00810 & 2311 & 0.00810 \\
\hline 513 & 0.04000 & 305 & 0.04600 & 3670 & 0.00020 & 2544 & 0.000208 \\
\hline sen & 0.05970 & 411 & 0.05070 & 0237 & 0.00040 & 4313 & 0.00940 \\
\hline 681 & 0.03540 & 471 & 0.03540 & 7203 & 0.00880 & 4001 & 0.00880 \\
\hline$m$ & 0.03030 & 500 & 0.03030 & 9156 & 0.00860 & 6331 & 0.00060 \\
\hline 881 & 0.02670 & $\infty$ & 0.02870 & 10350 & 0.00050 & 7143 & 0.00050 \\
\hline 1100 & 0.02100 & 787 & 0.02100 & 12554 & 0.00810 & 8681 & 0.00810 \\
\hline 1359 & 0.01720 & 940 & 0.01720 & 14181 & 0.00770 & 0806 & 0.00770 \\
\hline 1628 & 0.01450 & 1126 & 0.01450 & 19854 & 0.00725 & 13500 & 0.00725 \\
\hline 2023 & 0.01050 & 1537 & 0.01050 & 24247 & 0.00870 & 16766 & 0.00670 \\
\hline 2538 & 0.00050 & 1755 & 0.00050 & & & & \\
\hline
\end{tabular}

(b) Heat transfer data (note: numbers in brackets refer to figure numbers)

Smooth tube data; $\downarrow_{A}(9)=0.772, \downarrow_{A}(10)=1$ (unscaled, $R e_{m}=R e$ )

\begin{tabular}{|c|c|c|c|c|c|c|c|}
\hline $\mathrm{Ro}_{\mathrm{m}}(10)$ & $\mathrm{Nu}$ & $\mathbf{R o}_{m}(\theta)$ & $\mathrm{Nu}_{\mathrm{m}}$ & $\mathrm{Ao}_{\mathrm{m}}(10)$ & $\mathrm{Nu}$ & $R_{0}(9)$ & $\mathrm{Nu}_{\mathrm{m}}$ \\
\hline 1005 & 10.58 & 776 & 10.58 & 10643 & 33.82 & 0220 & 33.82 \\
\hline 1337 & 11.29 & 1033 & 1120 & 11487 & 35.91 & 8872 & 35.91 \\
\hline 1579 & 11.98 & 1210 & 11.98 & 13045 & 40.24 & 10075 & 40.24 \\
\hline 2044 & 12.25 & 1578 & 12.25 & 15860 & 46.63 & 12256 & 46.63 \\
\hline 2301 & 12.83 & $17 \pi$ & 12.83 & 18136 & 52.05 & 14007 & 52.05 \\
\hline $27 r 9$ & 13.12 & 2146 & 13.12 & 19939 & 56.94 & 15400 & 56.94 \\
\hline 3193 & 14.19 & 2466 & 14.19 & 21768 & 59.86 & 16813 & 59.86 \\
\hline 3743 & 18.30 & 2801 & 15.30 & 24131 & 66.77 & 18638 & 66.77 \\
\hline 4313 & 16.33 & 3031 & 16.33 & 27248 & 7260 & 21045 & 72.68 \\
\hline 4852 & 17.78 & 3747 & 17.78 & 30014 & 76.98 & 23181 & 76.93 \\
\hline 5321 & 10.20 & 4100 & 18.20 & 33371 & $\infty .60$ & 20001 & $\infty 0.60$ \\
\hline 5620 & 20.38 & 4340 & 20.36 & 39850 & 97.03 & 30785 & 97.03 \\
\hline 6745 & 23.49 & 8210 & 23.49 & 44468 & 108.16 & 34344 & 108.16 \\
\hline 7604 & 26.25 & $\operatorname{sens}$ & 26.25 & 48081 & 112.00 & 37830 & 112.80 \\
\hline 8683 & 27.69 & 6708 & 27.60 & 52066 & 115.58 & 40212 & 115.58 \\
\hline
\end{tabular}




\begin{tabular}{|c|c|c|c|c|c|c|c|}
\hline$R_{n_{m}}(10)$ & Nu & $\mathrm{Ro}_{m}(\theta)$ & $\mathrm{Nu}_{\mathrm{m}}$ & $A n_{m}(10)$ & $\mathrm{Nu}$ & $R o_{m}(\theta)$ & $\mathrm{Nu}_{\mathrm{m}}$ \\
\hline 8982 & 31.63 & 7840 & 31.83 & secos & 12025 & 45494 & 120.25 \\
\hline
\end{tabular}

$$
\beta=0.212, \downarrow_{A}(9)=0.731, \downarrow_{A}(10)=0.946
$$

\begin{tabular}{|c|c|c|c|c|}
\hline Re & $\mathrm{Nu}$ & $\mathbf{R \theta}_{m}(\boldsymbol{\theta})$ & $R e_{m}(10)$ & $N u_{m}$ \\
\hline 1072 & 8.61 & 783 & 1014 & 9.61 \\
\hline 1201 & 10.00 & 878 & 1137 & 10.00 \\
\hline 1337 & 10.23 & 978 & 1266 & 10.23 \\
\hline 1483 & 10.19 & 1084 & 1404 & 10.19 \\
\hline 1649 & 10.39 & 1205 & 1560 & 10.39 \\
\hline 1858 & 10.73 & 1358 & 1758 & 10.73 \\
\hline 1960 & 11.03 & 1433 & 1855 & 11.03 \\
\hline 2189 & 10.96 & 1600 & 2072 & 10.96 \\
\hline 2490 & 11.06 & 1820 & 2356 & 11.06 \\
\hline 2708 & 11.01 & 1979 & 2563 & 11.01 \\
\hline 3021 & 11.34 & 2208 & 2859 & 11.34 \\
\hline 3368 & 12.04 & 2462 & 3187 & 12.04 \\
\hline 3760 & 13.11 & 2748 & 3558 & 13.11 \\
\hline 4341 & 14.93 & 3173 & 4108 & 14.93 \\
\hline 4889 & 16.55 & 3574 & 4627 & 16.55 \\
\hline 5465 & 18.43 & 3994 & 5172 & 18.43 \\
\hline 6089 & 20.00 & 4450 & 5762 & 20.00 \\
\hline 6901 & 2250 & 5045 & 6532 & 22.50 \\
\hline 7559 & 24.50 & 5525 & 7153 & 24.50 \\
\hline 8481 & 27.06 & 6199 & 8026 & 27.06 \\
\hline 9235 & 28.75 & 6751 & 8740 & 28.75 \\
\hline 10931 & 33.04 & 7990 & 10345 & 33.04 \\
\hline 11919 & 35.77 & 8712 & 11280 & 35.77 \\
\hline 13493 & 39.46 & 9862 & 12769 & 39.46 \\
\hline 14957 & 42.58 & 10933 & 14155 & 42.58 \\
\hline
\end{tabular}




\begin{tabular}{|l|l|r|r|r|}
\hline$R_{\theta}$ & $N u$ & $R_{\theta_{m}}(\theta)$ & $R_{\theta_{m}}(10)$ & \multicolumn{1}{c|}{$N u_{m}$} \\
\hline 16528 & 47.49 & 12081 & 15642 & 47.49 \\
\hline 20623 & 55.26 & 15074 & 19517 & 55.26 \\
\hline 22876 & 59.43 & 16721 & 21649 & 59.43 \\
\hline 25995 & 65.61 & 19001 & 24602 & 65.61 \\
\hline 28799 & 70.47 & 21050 & 27255 & 70.47 \\
\hline 30925 & 75.55 & 22605 & 29267 & 75.55 \\
\hline 35729 & 83.66 & 26116 & 33814 & 83.66 \\
\hline 38563 & 89.81 & 28187 & 36496 & 89.81 \\
\hline 44581 & 99.98 & 32586 & 42191 & 99.98 \\
\hline 50276 & 110.22 & 36749 & 47581 & 110.22 \\
\hline
\end{tabular}

$$
\beta=0.409, \downarrow_{R}(9)=0.697, \downarrow_{A}(10)=0.902
$$

\begin{tabular}{|c|c|c|c|c|}
\hline $\mathbf{R e}_{\boldsymbol{\theta}}$ & $\mathrm{Nu}$ & $R e_{m}(9)$ & $R \theta_{m}(10)$ & $\mathrm{Nu}_{\mathrm{m}}$ \\
\hline 1062 & 8.01 & 740 & 958 & 8.01 \\
\hline 1256 & 8.29 & 875 & 1133 & 8.29 \\
\hline 1408 & 8.69 & 981 & 1270 & 8.69 \\
\hline 1568 & 8.53 & 1092 & 1414 & 8.53 \\
\hline 1661 & 8.70 & 1157 & 1499 & 8.70 \\
\hline 1858 & 8.83 & 1295 & 1676 & 8.83 \\
\hline 2039 & 8.92 & 1421 & 1839 & 8.92 \\
\hline 2288 & 8.98 & 1594 & 2064 & 8.98 \\
\hline 2598 & 9.15 & 1810 & 2344 & 9.15 \\
\hline 2922 & 9.37 & 2036 & 2636 & 9.37 \\
\hline 3205 & 10.96 & 2233 & 2891 & 10.96 \\
\hline 3580 & 1214 & 2494 & 3229 & 12.14 \\
\hline 4216 & 14.11 & 2937 & 3803 & 14.11 \\
\hline 4736 & 15.86 & 3300 & 4272 & 15.86 \\
\hline 5207 & 16.99 & 3628 & 4697 & 16.99 \\
\hline 5809 & 19.22 & 4047 & 5240 & 19.22 \\
\hline
\end{tabular}




\begin{tabular}{|c|c|c|c|c|}
\hline Ro & Nu & $R \boldsymbol{e}_{m}(\boldsymbol{\theta})$ & $R \theta_{m}(10)$ & $N u_{m}$ \\
\hline 6517 & 21.22 & 4541 & 5880 & 21.22 \\
\hline 7197 & 23.64 & 5015 & 6493 & 23.64 \\
\hline 8035 & 25.64 & 5598 & 7248 & 25.64 \\
\hline 9055 & 27.78 & 6309 & 8169 & 27.78 \\
\hline 10160 & 30.86 & 7079 & 9166 & 30.86 \\
\hline 11575 & 34.44 & 8065 & 10442 & 34.44 \\
\hline 12889 & 37.53 & 8980 & 11627 & 37.53 \\
\hline 14206 & 39.48 & 9898 & 12816 & 39.48 \\
\hline 15053 & 40.87 & 10488 & 13579 & 40.87 \\
\hline 15638 & 43.76 & 10896 & 14107 & 43.76 \\
\hline 17513 & 47.09 & 12202 & 15799 & 47.09 \\
\hline 19438 & 51.38 & 13544 & 17536 & 51.38 \\
\hline 20953 & 55.69 & 14599 & 18903 & 55.69 \\
\hline 24627 & 60.92 & 17159 & 22217 & 60.92 \\
\hline 27196 & 66.46 & 18949 & 24534 & 66.46 \\
\hline 30072 & 70.79 & 20952 & 27128 & 70.79 \\
\hline 32789 & 75.94 & 22846 & 29580 & 75.94 \\
\hline 36071 & 81.41 & 25133 & 32541 & 81.41 \\
\hline 40705 & 88.25 & 28361 & 36721 & 88.25 \\
\hline
\end{tabular}

$$
\beta=0.6, \downarrow_{A}(9)=0.648, \downarrow_{R}(10)=0.839
$$

\begin{tabular}{|c|c|c|c|c|}
\hline$R \boldsymbol{R}$ & $\mathrm{Nu}$ & $R \theta_{m}(9)$ & $R \theta_{m}(10)$ & $\mathrm{Nu}_{m}$ \\
\hline 1328 & 6.40 & 861 & 1115 & 6.40 \\
\hline 1658 & 6.74 & 1075 & 1392 & 6.74 \\
\hline 2070 & 7.12 & 1342 & 1738 & 7.12 \\
\hline 2499 & 7.34 & 1620 & 2098 & 7.34 \\
\hline 2844 & 7.78 & 1844 & 2387 & 7.78 \\
\hline 3116 & 8.38 & 2020 & 2616 & 8.38 \\
\hline 3693 & 11.44 & 2394 & 3100 & 11.44 \\
\hline
\end{tabular}




\begin{tabular}{|c|c|c|c|c|}
\hline Re & $\mathrm{Nu}$ & $R e_{m}(\theta)$ & $R e_{m}(10)$ & $\mathrm{Nu}_{\mathrm{m}}$ \\
\hline 4081 & 13.12 & 2646 & 3426 & 13.12 \\
\hline 4715 & 15.06 & 3057 & 3958 & 15.06 \\
\hline 5220 & 16.65 & 3384 & 4382 & 16.65 \\
\hline 5876 & 18.19 & 3809 & 4932 & 18.18 \\
\hline 6447 & 19.72 & 4180 & 5412 & 19.72 \\
\hline 7106 & 21.55 & 4607 & 5965 & 21.55 \\
\hline 8600 & 25.76 & 5576 & 7220 & 25.76 \\
\hline 10057 & 28.97 & 6521 & 8443 & 28.97 \\
\hline 12653 & 34.62 & 8204 & 10622 & 34.62 \\
\hline 14053 & 38.35 & 9111 & 11797 & 38.35 \\
\hline 15062 & 41.08 & 9765 & 12644 & 41.08 \\
\hline 15838 & 42.41 & 10269 & 13296 & 42.41 \\
\hline 18770 & 48.52 & 12169 & 15757 & 48.52 \\
\hline 20156 & 52.08 & 13068 & 16920 & 52.08 \\
\hline 21548 & 54.37 & 13971 & 18089 & 54.37 \\
\hline 23781 & 57.53 & 15418 & 19963 & 57.53 \\
\hline 25929 & 61.36 & 16811 & 21767 & 61.36 \\
\hline 27720 & 64.51 & 17972 & 23270 & 64.51 \\
\hline 29710 & 68.08 & 19263 & 24941 & 68.08 \\
\hline 31762 & 72.51 & 20593 & 26663 & 72.51 \\
\hline 33463 & 74.96 & 21696 & 28091 & 74.96 \\
\hline
\end{tabular}

$$
\beta=0.698, \downarrow_{A}(9)=0.618, \downarrow_{R}(10)=0.800
$$

\begin{tabular}{|c|r|r|r|r|}
\hline$R e$ & $N u$ & $R e_{m}(\theta)$ & $R e_{m}(10)$ & \multicolumn{1}{c|}{$N u_{m}$} \\
\hline 3317 & 8.10 & 2050 & 2654 & 8.10 \\
\hline 3861 & 11.36 & 2386 & 3090 & 11.36 \\
\hline 4447 & 13.58 & 2748 & 3558 & 13.58 \\
\hline 4942 & 15.01 & 3054 & 3955 & 15.01 \\
\hline 5327 & 16.20 & 3292 & 4263 & 16.20 \\
\hline
\end{tabular}




\begin{tabular}{|c|r|r|r|r|}
\hline$R_{\theta}$ & \multicolumn{1}{|c|}{$N u$} & $R_{\theta_{m}}(9)$ & $R_{\theta_{m}}(10)$ & \multicolumn{1}{c|}{$N u_{m}$} \\
\hline 6058 & 18.21 & 3744 & 4847 & 18.21 \\
\hline 6810 & 20.25 & 4209 & 5449 & 20.25 \\
\hline 7853 & 23.02 & 4853 & 6284 & 23.02 \\
\hline 8573 & 24.55 & 5298 & 6860 & 24.55 \\
\hline 8674 & 27.42 & 5979 & 7741 & 27.42 \\
\hline 10462 & 28.75 & 6465 & 8371 & 28.75 \\
\hline 11716 & 31.71 & 7240 & 8375 & 31.71 \\
\hline 13045 & 34.56 & 8062 & 10439 & 34.56 \\
\hline 15091 & 38.55 & 9326 & 12075 & 38.55 \\
\hline 16921 & 43.13 & 10458 & 13540 & 43.13 \\
\hline
\end{tabular}

$\beta=0.793, \downarrow_{R}(9)=0.692, \downarrow_{R}(10)=0.895$

\begin{tabular}{|c|c|c|c|c|}
\hline Re & $\mathrm{Nu}$ & $R \theta_{m}(9)$ & $\mathrm{Re} e_{\mathrm{m}}(10)$ & $N u_{m}$ \\
\hline 2528 & 6.21 & 1748 & 2263 & 6.21 \\
\hline 2806 & 7.05 & 1940 & 2512 & 7.05 \\
\hline 2937 & 7.63 & 2031 & 2630 & 7.63 \\
\hline 3302 & 9.81 & 2283 & 2956 & 9.81 \\
\hline 3729 & 11.05 & 2578 & 3338 & 11.05 \\
\hline 4123 & 12.28 & 2851 & 3691 & 12.28 \\
\hline 4769 & 14.13 & 3298 & 4270 & 14.13 \\
\hline 5486 & 15.97 & 3793 & 4911 & 15.97 \\
\hline 6505 & 18.61 & 4498 & 5824 & 18.61 \\
\hline 6972 & 19.68 & 4821 & 6242 & 19.68 \\
\hline 8106 & 22.41 & 5605 & 7258 & 22.41 \\
\hline 9090 & 24.82 & 6285 & 8138 & 24.82 \\
\hline 9880 & 26.51 & 6831 & 8845 & 26.51 \\
\hline 11022 & 29.01 & 7621 & 9868 & 29.01 \\
\hline 12856 & 32.85 & 8889 & 11510 & 32.85 \\
\hline 13858 & 34.74 & 9582 & 12407 & 34.74 \\
\hline
\end{tabular}


Table A.7

Koch (1960)

Friction and heat transfer data for smooth and rough tubes

(a) Smooth tube

Friction (smooth): $\downarrow_{A}=0.713, \downarrow_{1}=1.0$

\begin{tabular}{|c|c|c|c|c|c|c|c|}
\hline Ra & 1 & $n_{m}$ & $t_{n}$ & An & 1 & $\mathbf{R o}_{m}$ & $t_{m}$ \\
\hline 286 & 0.05080 & 204 & 0.05080 & 6081 & 0.00000 & 3024 & 0.00960 \\
\hline 341 & 0.05187 & 243 & 0.05187 & 5414 & 0.00040 & $3 e 61$ & 0.00940 \\
\hline 307 & 0.04006 & 283 & 0.04023 & $\operatorname{sen} 2$ & 0.00013 & 4181 & 0.00913 \\
\hline 483 & 0.03180 & 345 & 0.03100 & 6840 & 0.00902 & 4622 & 0.00902 \\
\hline 600 & 0.02554 & 434 & 0.02564 & 6763 & 0.00099 & 4824 & 0.00899 \\
\hline 657 & 0.02183 & 468 & 0.02183 & 7489 & 0.00877 & 8341 & 0.00877 \\
\hline 692 & 0.02107 & 493 & 0.02107 & 8149 & 0.00850 & 5813 & 0.00859 \\
\hline 733 & 0.02236 & 523 & 0.00236 & 8715 & 0.00834 & 6216 & 0.00834 \\
\hline 833 & 0.01890 & 504 & 0.01693 & e285 & 0.00824 & 6023 & 0.00824 \\
\hline 806 & 0.01600 & 659 & 0.01600 & 10243 & 0.00825 & 7306 & 0.00825 \\
\hline 853 & 0.01646 & 600 & 0.01646 & 10887 & 0.00796 & 7765 & 0.00796 \\
\hline 1060 & 0.01478 & 758 & 0.01478 & 11847 & 0.00792 & 8450 & 0.00792 \\
\hline 1171 & 0.01373 & 835 & 0.01373 & 12638 & 0.00778 & 8014 & 0.00778 \\
\hline 1260 & 0.01278 & $\cos$ & 0.01278 & 13821 & 0.00758 & 8058 & 0.00758 \\
\hline 1354 & 0.01227 & 966 & 0.01227 & 14744 & 0.00750 & 10517 & 0.00750 \\
\hline 1475 & 0.01220 & 1052 & 0.01220 & 15554 & 0.00731 & 11004 & 0.00731 \\
\hline 1552 & 0.01100 & 1107 & 0.01100 & 17635 & 0.00711 & 12578 & 0.00711 \\
\hline 1643 & 0.01070 & 1172 & 0.01070 & 20702 & 0.00603 & 14766 & 0.00593 \\
\hline 1717 & 0.01018 & 1224 & 0.01019 & 22781 & 0.00679 & 16249 & 0.00679 \\
\hline 1820 & 0.00009 & 1305 & 0.00909 & 25352 & 0.00659 & 18083 & 0.00650 \\
\hline 1946 & 0.000020 & 1388 & 0.00020 & 28565 & 0.00638 & 20375 & 0.00638 \\
\hline 2007 & 0.00041 & 1408 & 0.00941 & 50434 & 0.00628 & 21708 & 0.00020 \\
\hline 217 & 0.00000 & 1553 & 0.00000 & 32547 & 0.00024 & 23215 & 0.00524 \\
\hline 2008 & 0.00837 & 1711 & 0.00837 & 35243 & 0.00607 & 25138 & 0.00607 \\
\hline 2020 & 0.00825 & 1871 & 0.00025 & 36947 & 0.00603 & 26354 & 0.00603 \\
\hline 2794 & 0.00855 & 1993 & 0.00855 & 39502 & 0.00595 & 28218 & 0.00595 \\
\hline 2944 & 0.00808 & 2100 & 0.00806 & 41579 & 0.00575 & 29657 & 0.00575 \\
\hline
\end{tabular}




\begin{tabular}{|c|c|c|c|c|c|c|c|}
\hline Re & 1 & $\mathbf{R}_{\boldsymbol{m}_{m}}$ & $t_{m}$ & Ro & 1 & $\boldsymbol{R}_{\mathrm{m}}$ & $f_{m}$ \\
\hline $\operatorname{sos} 0$ & 0.00881 & ₹161 & 0.00881 & 4854 & 0.00072 & 31003 & 0.00572 \\
\hline 9180 & 0.00893 & 20.8 & 0.00083 & 48088 & 0.00550 & 34500 & 0.00559 \\
\hline 3285 & 0.00045 & 2018 & 0.00045 & 53646 & 0.00548 & 58265 & 0.00546 \\
\hline $\sec 2$ & 0.00080 & 200 & 0.00880 & 88161 & 0.00580 & 41485 & 0.00530 \\
\hline 3458 & 0.01055 & 2485 & 0.01055 & 60098 & 0.00534 & 43437 & 0.00534 \\
\hline 9814 & 0.01002 & 20078 & 0.01002 & 63844 & 0.00550 & 45538 & 0.00530 \\
\hline 3841 & 0.01024 & 2740 & 0.01024 & 67182 & $0.0052 x$ & 47919 & 0.00523 \\
\hline 4175 & 0.01005 & 2078 & 0.01005 & 74762 & 0.00508 & 53526 & 0.00506 \\
\hline 4760 & 0.00070 & 3402 & 0.00970 & 80452 & 0.00508 & 57384 & 0.00506 \\
\hline
\end{tabular}

Heat transfer (smooth):

\begin{tabular}{|c|c|c|c|}
\hline$R e$ & $\mathbf{N} \mathbf{u}_{\mathrm{m}}$ & $\mathbf{R e}$ & $\mathbf{N u} u_{m}$ \\
\hline 447 & 6.49 & 5703 & 21.29 \\
\hline 526 & 7.69 & 5999 & 21.13 \\
\hline 596 & 8.43 & 6670 & 23.77 \\
\hline 678 & 9.19 & 7704 & 26.20 \\
\hline 730 & 10.01 & 8566 & 28.06 \\
\hline 787 & 9.81 & 9477 & 30.58 \\
\hline 819 & 10.83 & 10146 & 32.05 \\
\hline 889 & 11.12 & 10918 & 33.92 \\
\hline 1112 & 11.42 & 11484 & 35.10 \\
\hline 1181 & 11.71 & 11942 & 36.37 \\
\hline 1235 & 12.11 & 12404 & 38.11 \\
\hline 1356 & 1200 & 13313 & 39.94 \\
\hline 1548 & 12.45 & 14362 & 41.49 \\
\hline 1704 & 12.53 & 16214 & 46.03 \\
\hline 1813 & 12.51 & 18281 & 50.16 \\
\hline 1949 & 12.85 & 19896 & 54.74 \\
\hline 2039 & 12.88 & 23211 & 60.65 \\
\hline 2203 & 12.82 & 26305 & 66.52 \\
\hline 2308 & 13.00 & 29103 & 72.50 \\
\hline
\end{tabular}




\begin{tabular}{|l|l|l|l|}
\hline$R e$ & $N u_{m}$ & $R e$ & $N u_{m}$ \\
\hline 2388 & 13.30 & 32732 & 79.22 \\
\hline 2487 & 13.46 & 34822 & 84.72 \\
\hline 2592 & 13.84 & 38185 & 90.26 \\
\hline 2689 & 13.76 & 40063 & 83.87 \\
\hline 2776 & 14.29 & 42248 & 99.12 \\
\hline 3002 & 14.62 & 45574 & 102.05 \\
\hline 3317 & 15.40 & 48913 & 106.53 \\
\hline 3735 & 15.97 & 50357 & 111.78 \\
\hline 4034 & 16.70 & 53035 & 115.82 \\
\hline 4368 & 17.52 & 56636 & 121.68 \\
\hline 4624 & 17.95 & 59119 & 124.01 \\
\hline 4882 & 19.00 & 61402 & 127.19 \\
\hline 5064 & 19.66 & 63615 & 131.45 \\
\hline 5260 & 19.88 & 66237 & 134.82 \\
\hline 5422 & 20.84 & 68713 & 139.86 \\
\hline & & & \\
\hline
\end{tabular}

(b) transverse disc-shaped inserts with no clearance at the wall

Friction $(\theta): p / e=3.92, \theta / D=0.1, \downarrow_{A}=2.061, \downarrow_{1}=0.173$

\begin{tabular}{|c|c|c|c|c|c|c|c|}
\hline Re & 1 & $\mathrm{P}_{\mathrm{om}_{\mathrm{m}}}$ & $t_{m}$ & Ro & $i$ & $R_{\boldsymbol{e}_{m}}$ & $t_{m}$ \\
\hline 403 & 0.08876 & 850 & 0.01713 & 4257. & 0.16004 & 8773 & 0.02775 \\
\hline 494 & 0.07901 & 1018 & 0.01370 & 5021 & 0.15427 & 10760 & 0.02675 \\
\hline 573 & 0.06810 & 1181 & 0.01183 & 6171 & 0.15138 & 12718 & 0.02525 \\
\hline 703 & 0.05868 & 1448 & 0.01018 & 8008 & 0.14850 & 16493 & 0.02575 \\
\hline 800 & 0.05450 & 1640 & 0.00045 & 0458 & 0.13985 & 19493 & 0.02425 \\
\hline 811 & 0.04888 & 1878 & 0.00848 & 11388 & 0.132293 & 23471 & 0.02305 \\
\hline 1018 & 0.04614 & 2100 & 0.00800 & 14231 & 0.13048 & 29320 & 0.02263 \\
\hline 1182 & 0.00934 & 2438 & 0.01723 & 15908 & 0.13293 & 32786 & 0.02305 \\
\hline 1423 & 0.13048 & 2933 & 0.02203 & 19513 & 0.13293 & 40216 & 0.02305 \\
\hline 1713 & 0.16580 & 3531 & 0.02875 & 23494 & 0.13293 & 48422 & 0.02305 \\
\hline 2102 & 0.17878 & 4332 & 0.03100 & 30470 & 0.13985 & 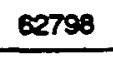 & 0.02425 \\
\hline
\end{tabular}




\begin{tabular}{|c|c|c|c|c|c|c|c|}
\hline $\mathbf{R o}$ & 1 & $\mathbf{P}_{m}$ & $t_{m}$ & Ro & 1 & $\boldsymbol{R}_{\boldsymbol{m}}$ & $f_{m}$ \\
\hline 2626 & 0.17878 & 8413 & 0.03100 & 38282 & 0.14273 & 78899 & 0.02475 \\
\hline 5047 & 0.17878 & 6200 & 0.03100 & 48471 & 0.14850 & 00890 & 0.02575 \\
\hline 3343 & 0.16580 & 6800 & 0.02875 & 50456 & 0.14850 & 122530 & 0.02575 \\
\hline
\end{tabular}

Heat transfer $(\theta): \downarrow_{R}=2889$

\begin{tabular}{|c|c|c|c|c|c|c|c|}
\hline Po & $\mathrm{Nu}$ & $\boldsymbol{P o}_{\mathrm{m}}$ & $N_{u_{m}}$ & Do & Nu & $\mathbf{R o}_{\mathrm{m}}$ & $\mathrm{Nu}_{m}$ \\
\hline 960 & 13.2 & $2 \pi / 2$ & 132 & 9820 & $\pi$ & 28309 & $\pi$ \\
\hline 1247 & 15.2 & $\sec 3$ & 15.2 & 13220 & 91.7 & 38196 & 91.7 \\
\hline 1650 & 20.1 & 4767 & 20.1 & 17477 & 114.9 & 60485 & 114.8 \\
\hline 2145 & 25.2 & 6188 & 25.2 & 22311 & 132 & 64462 & 132 \\
\hline 2789 & 31.1 & 8057 & 31.1 & 27990 & 168.2 & 80871 & 168.2 \\
\hline 3501 & 37 & 10115 & 37 & 32181 & 186.6 & 82981 & 186.6 \\
\hline 4797 & 44.8 & 13850 & 44.8 & 43291 & 233.8 & 125080 & 233.8 \\
\hline 5754 & 51.5 & 16525 & 51.5 & 50238 & 287.8 & 162487 & 287.8 \\
\hline 7612 & 64.7 & 21994 & 64.7 & & & & \\
\hline
\end{tabular}

Stanton number ratios (e):

\begin{tabular}{|c|c|c|c|c|}
\hline $\mathbf{R}_{m}$ & $f_{m}$ & $e^{+}$ & $\boldsymbol{e}_{m}{ }^{+}$ & $84 / s_{2}$ \\
\hline 1877 & 0.00779 & 14.38 & 12.34 & 120 \\
\hline 2570 & 0.01991 & 29.88 & 25.64 & 125 \\
\hline 3400 & 0.02745 & 46.42 & 39.83 & 1.60 \\
\hline 4421 & 0.050098 & 64.12 & 55.02 & 1.97 \\
\hline 5747 & 0.03128 & es.76 & 71.87 & 2.16 \\
\hline 7215 & 0.02788 & 90.27 & 85.18 & 2.36 \\
\hline 8885 & 0.02730 & 134.82 & 115.68 & 2.41 \\
\hline 11858 & 0.02634 & 158.50 & 136.08 & 2.42 \\
\hline 15688 & 0.02508 & 206.30 & 178.81 & 2.48 \\
\hline 20258 & 0.00302 & 258.16 & 221.51 & 2.46 \\
\hline 27244 & 0.02250 & 397.47 & 209.56 & 250 \\
\hline 36017 & 0.02319 & 452.00 & 387.83 & 237 \\
\hline 45979 & 0.02298 & 574.35 & 492.81 & 2.23 \\
\hline 57683 & 0.02381 & 733.47 & 620.34 & 2.39 \\
\hline
\end{tabular}




\begin{tabular}{|c|c|c|c|c|}
\hline $\boldsymbol{n}_{\boldsymbol{e}_{m}}$ & in & $0^{+}$ & $a_{m}^{+}$ & $8, s_{3}$ \\
\hline cesen & 0.02444 & 0.54 .53 & 733.21 & 2.30 \\
\hline 60218 & 0.00510 & 1186.88 & 100122 & 2.32 \\
\hline 115000 & 0.00805 & 1541.50 & 182073 & 238 \\
\hline
\end{tabular}

Friction $(1): p / e=8.8, Q / D=0.1, t_{A}=2161, t_{f}=0.217$

\begin{tabular}{|c|c|c|c|c|c|c|c|}
\hline Po & 1 & $\mathbf{P}_{m}$ & $t_{m}$ & no & 1 & $\mathbf{R e}_{m}$ & in \\
\hline 274 & 0.10627 & 602 & 0.02508 & 8056 & 0.15774 & 8504 & 0.03426 \\
\hline 392 & 0.00000 & 717 & 0.01881 & 4021 & 0.15744 & 8552 & 0.03420 \\
\hline 990 & 0.06087 & 881 & 0.01518 & 40028 & 0.15744 & 10647 & 0.03420 \\
\hline 453 & 0.06743 & 979 & 0.01485 & 5578 & 0.15385 & 12052 & 0.03344 \\
\hline 544 & 0.05740 & 1174 & 0.01247 & 5093 & 0.15598 & 12047 & 0.03388 \\
\hline 631 & 0.05073 & 1563 & 0.01102 & 6705 & 0.15424 & 14487 & 0.03350 \\
\hline 600 & 0.04760 & 1490 & 0.01034 & 7860 & 0.15194 & 16981 & 0.03300 \\
\hline 823 & 0.04160 & 1778 & 0.00504 & 9484 & 0.15337 & 20491 & 0.03331 \\
\hline 972 & 0.00683 & 2100 & 0.00800 & 10571 & 0.15184 & 22830 & 0.03300 \\
\hline 1024 & 0.03896 & 2213 & 0.00846 & 11866 & 0.15081 & 25853 & 0.03276 \\
\hline 1003 & 0.04841 & 22006 & 0.01051 & 13546 & 0.15166 & 20266 & 0.03294 \\
\hline 1107 & 0.05708 & 2001 & 0.01240 & 15453 & 0.15100 & 30386 & 0.03282 \\
\hline 1189 & 0.10607 & 2060 & 0.02504 & 17124 & 0.15300 & 36096 & 0.03325 \\
\hline 1270 & 0.12766 & 274 & 0.02773 & 19300 & 0.15081 & 41717 & 0.03276 \\
\hline 1386 & 0.14311 & 2093 & 0.03108 & 22721 & 0.15598 & 49089 & 0.03388 \\
\hline 1512 & 0.15366 & 3288 & 0.03337 & 27250 & 0.15863 & 58893 & 0.03445 \\
\hline 1618 & 0.15863 & suss & 0.03445 & 28556 & 0.16163 & 61695 & 0.05510 \\
\hline 1745 & 0.15863 & 3770 & 0.03445 & 31851 & 0.16023 & 00031 & 0.03524 \\
\hline 1874 & 0.16254 & 4050 & 0.03530 & 36008 & 0.16468 & 7891 & 0.05577 \\
\hline 1860 & 0.16406 & 4204 & 0.03563 & 38407 & $0.16 \times 23$ & 82979 & 0.05610 \\
\hline 2163 & 0.16468 & 4874 & 0.03577 & 41743 & 0.17128 & 80186 & 0.03720 \\
\hline 2449 & 0.16499 & 5201 & 0.03584 & 45106 & 0.16937 & 97452 & 0.05879 \\
\hline 2656 & 0.16437 & 5730 & 0.03570 & 52150 & 0.17205 & 112691 & 0.05741 \\
\hline 2093 & 0.16163 & 60 & 0.03510 & 58361 & 0.17485 & 126091 & 0.03788 \\
\hline 3066 & 0.16183 & 6024 & 0.03517 & 65048 & 0.17815 & 140538 & 0.03869 \\
\hline 347 & 0.15863 & 7512 & 0.03445 & 70836 & 0.18118 & 153042 & 0.03935 \\
\hline
\end{tabular}


Heat transfer $(i): \downarrow_{A}=3.020$

\begin{tabular}{|c|c|c|c|c|c|c|c|}
\hline A & $\mathrm{Nu}$ & $\mathbf{n}_{m}$ & $\mathrm{Nu}_{\mathrm{m}}$ & Po & $\mathrm{Nu}$ & $\mathbf{n}_{\mathrm{m}}$ & $N u_{m}$ \\
\hline 408 & 8.00 & 1257 & 5.00 & 8028 & 4420 & 10038 & 44.20 \\
\hline 401 & 6.83 & 1488 & 6.03 & 4404 & 40.41 & 13340 & 40.41 \\
\hline 881 & 8.01 & 1000 & 8.01 & 5056 & 85.34 & 150254 & 8.34 \\
\hline 637 & 0.14 & 1000 & 0.14 & 6514 & 58.16 & 16701 & 68.18 \\
\hline 785 & 10.51 & 2016 & 10.51 & 6169 & 60.43 & 18687 & 03.43 \\
\hline 870 & 1154 & 2034 & 11.54 & 7145 & 60.55 & 21642 & 60.55 \\
\hline 922 & 11.70 & 2002 & 11.70 & 8611 & 79.57 & 20084 & 70.57 \\
\hline$\infty 39$ & 12.43 & 2043 & 1243 & 10492 & $\propto 3.68$ & 31781 & es.e6 \\
\hline 1028 & 13.73 & 3112 & 13.73 & 11890 & 100.36 & 30014 & 100.38 \\
\hline 1123 & 15.03 & 3402 & 15.03 & 13971 & 115.63 & 42318 & 115.63 \\
\hline 1188 & 16.02 & 3508 & 16.02 & 16536 & 129.97 & 50083 & 129.97 \\
\hline 1238 & 17.01 & 3751 & 17.01 & 10642 & 150.82 & 59497 & 150.82 \\
\hline 1303 & 18.20 & 3047 & 18.20 & 23460 & 171.84 & 71081 & 171.84 \\
\hline 1401 & 19.29 & 1243 & 19.20 & 27219 & 190.88 & 82446 & 190.88 \\
\hline 1579 & 21.76 & 4782 & 21.76 & 28844 & 199.88 & 87369 & 199.88 \\
\hline 1732 & 28.73 & 5246 & 23.73 & 32568 & 218.78 & $\operatorname{ces} 40$ & 218.78 \\
\hline 1003 & 25.21 & 5764 & 25.21 & 36441 & 239.46 & 110380 & 200.46 \\
\hline 2184 & 29.51 & 6815 & 29.51 & 38898 & 253.87 & 117822 & 253.87 \\
\hline 2548 & 32.82 & $\pi 17$ & 32.82 & 42356 & 269.15 & 128298 & 269.15 \\
\hline 2734 & 35.42 & 8282 & 35.42 & 49680 & 303.07 & 150482 & 303.07 \\
\hline 3110 & 39.11 & 9419 & 39.11 & 60861 & 344.90 & 184349 & 344.90 \\
\hline
\end{tabular}

Stanton number ratios (f):

\begin{tabular}{|c|c|c|c|c|}
\hline$R \boldsymbol{\theta}_{\mathrm{m}}$ & $f_{m}$ & $0^{+}$ & $a^{+}$ & st/st \\
\hline 883 & 0.01493 & 7.57 & 7.62 & 1.01 \\
\hline 1000 & 0.01397 & 8.80 & 8.88 & 0.85 \\
\hline 1212 & 0.01207 & 0.35 & 0.42 & 0.00 \\
\hline 1376 & 0.01005 & 10.11 & 10.18 & 1.05 \\
\hline 1652 & 0.00958 & 11.34 & 11.42 & 1.06 \\
\hline 1879 & 0.00868 & 12.30 & 12.38 & 1.12 \\
\hline 2013 & 0.00828 & 12.85 & 12.94 & 1.08 \\
\hline
\end{tabular}




\begin{tabular}{|c|c|c|c|c|}
\hline $\boldsymbol{n}_{m}$ & $t_{m}$ & $e^{+}$ & $a_{m}^{+}$ & $8, / 8 x_{2}$ \\
\hline 20000 & 0.00022 & 12.91 & 13.00 & 1.13 \\
\hline 200 & 0.00881 & 14.46 & 14.56 & 122 \\
\hline 2426 & 0.013008 & 20.14 & 20.28 & 1.31 \\
\hline 2586 & 0.00002 & 2720 & 27.47 & 1.36 \\
\hline 2876 & 0.02684 & 80.67 & $\mathbf{8 0 . 8 8}$ & 1.40 \\
\hline 2815 & 0.02875 & 33.52 & 29.75 & 1.50 \\
\hline 3027 & 0.00144 & 37.60 & 37.85 & 1.60 \\
\hline 2411 & 0.03419 & 44.30 & 44.60 & 1.74 \\
\hline 3742 & 0.00444 & 48.76 & 49.10 & 1.00 \\
\hline 4111 & 0.03545 & 54.36 & 54.74 & 1.98 \\
\hline 4718 & 0.03577 & 62.67 & 03.10 & 2.30 \\
\hline 5504 & 0.03583 & 73.17 & 73.68 & 2.39 \\
\hline 5007 & 0.03549 & 78.15 & 78.69 & 2.53 \\
\hline 6719 & 0.03516 & 88.47 & 80.08 & 2.64 \\
\hline 7038 & 0.03429 & 101.82 & 102.63 & 2.79 \\
\hline 9515 & 0.03420 & 123.57 & 124.42 & 2.81 \\
\hline 10880 & 0.03407 & 141.04 & 142.02 & 2.82 \\
\hline 11912 & 0.03844 & 152.99 & 154.04 & 274 \\
\hline 13320 & 0.03304 & 172.44 & 173.63 & 2.85 \\
\hline 15437 & 0.03317 & 197.42 & 188.79 & 2.75 \\
\hline 18605 & 0.03316 & 237.80 & 239.54 & 2.82 \\
\hline 22668 & 0.03503 & 209.32 & 291.31 & 2.85 \\
\hline 25688 & $0.0<275$ & 326.48 & 328.73 & $2 \pi$ \\
\hline $\mathbf{5 0 1 8 4}$ & 0.05202 & 384.58 & 387.23 & 2.83 \\
\hline 35725 & 0.03313 & 456.68 & 450.83 & 2.78 \\
\hline 42438 & 0.03275 & 550.38 & 543.08 & 2.70 \\
\hline 50686 & 0.03408 & 656.62 & 661.15 & 2.82 \\
\hline 58807 & 0.03444 & 768.37 & 771.68 & 2.78 \\
\hline 62318 & 0.03521 & 821.17 & 828.84 & 2.78 \\
\hline 70564 & 0.03523 & 827.76 & 034.16 & 278 \\
\hline 78732 & 0.03581 & 1048.20 & 1053.51 & 2.73 \\
\hline 84040 & 0.03626 & 1123.75 & 1131.51 & 2.78 \\
\hline 91512 & 0.03723 & 1240.05 & 1248.61 & 2.71 \\
\hline
\end{tabular}




\begin{tabular}{|c|c|c|c|c|}
\hline $\mathbf{P}_{m}$ & $t_{m}$ & $0^{+}$ & $a_{m}^{+}$ & expst \\
\hline 107385 & 0.05600 & 1449.80 & 1450.81 & $2 \pi$ \\
\hline 131491 & 0.08821 & 1805.04 & 1817.50 & 273 \\
\hline
\end{tabular}

Friction $(g): p / \theta=65, \theta / D=0.1, \downarrow_{A}=2813, \downarrow_{f}=0.215$

\begin{tabular}{|c|c|c|c|c|c|c|c|}
\hline Po & 1 & $\mathbf{R o}_{\mathrm{m}}$ & in & no & $f$ & $\mathbf{R o}_{\mathrm{m}}$ & $t_{m}$ \\
\hline 500 & 0.04525 & 1405 & 0.00978 & 2428 & 0.05027 & 6009 & 0.01087 \\
\hline 853 & 0.04178 & 1584 & 0.00003 & 3110 & 0.05015 & 8748 & 0.01084 \\
\hline 637 & 0.04063 & 1791 & 0.00878 & 3790 & 0.04973 & 10687 & 0.01075 \\
\hline 747 & 0.03718 & 2100 & 0.00804 & 5064 & 0.05161 & 14245 & 0.01116 \\
\hline 818 & 0.04127 & 2002 & 0.00892 & 6488 & 0.05137 & 16246 & 0.01111 \\
\hline 844 & 0.04657 & 2857 & 0.01007 & 8656 & 0.05242 & 24351 & 0.01133 \\
\hline 1004 & 0.04801 & 2064 & 0.01057 & 11452 & 0.05242 & 32216 & 0.01133 \\
\hline 1165 & 0.04967 & 8278 & 0.01074 & 14834 & 0.05223 & 41720 & 0.01129 \\
\hline 1268 & 0.05027 & 3568 & 0.01087 & 18625 & 0.05267 & 55207 & 0.01130 \\
\hline 1403 & 0.05088 & 3046 & 0.01100 & 26191 & 0.05318 & 73677 & 0.01150 \\
\hline 1480 & 0.05059 & 4163 & 0.01006 & 35347 & 0.05545 & 99433 & 0.01189 \\
\hline 1821 & 0.05045 & 5405 & 0.01091 & 47114 & 0.05652 & 132534 & 0.01222 \\
\hline
\end{tabular}

Heat transfer $(g): \downarrow_{A}=3.944$

\begin{tabular}{|c|c|c|c|c|c|c|c|}
\hline Re & $\mathrm{Nu}$ & $\mathbf{R o}_{\mathrm{m}}$ & $\mathrm{Nu}_{\mathrm{m}}$ & Ro & Nu & $\mathbf{R o}_{\mathrm{m}}$ & $\mathrm{Nu}_{\mathrm{m}}$ \\
\hline 1205 & 12.80 & 4751 & 12.60 & 7440 & 46.99 & 29344 & 46.99 \\
\hline 1511 & 15.31 & 5050 & 15.31 & 10108 & 61.00 & 39865 & 61.00 \\
\hline 2193 & 18.76 & 8647 & 18.76 & 13071 & 73.98 & 51550 & 73.98 \\
\hline 2476 & 20.49 & 9765 & 20.49 & 17161 & 9121 & 67679 & 91.21 \\
\hline 2740 & 22.58 & 10006 & 22.58 & 22816 & 112.87 & 89184 & 112.87 \\
\hline 3020 & 25.55 & 12707 & 25.55 & 20843 & 139.68 & 117686 & 139.68 \\
\hline 4433 & 33.01 & 17483 & 33.01 & 30380 & 172.85 & 155308 & 172.85 \\
\hline 5653 & 39.83 & 22004 & $\$ 9.03$ & & & & \\
\hline
\end{tabular}


Stanton number ratios (g):

\begin{tabular}{|c|c|c|c|c|}
\hline $\boldsymbol{R}_{m}$ & 4 & $0^{+}$ & $a_{m}{ }^{+}$ & eqst \\
\hline 2000 & 0.01073 & 10.02 & 24.82 & 1.08 \\
\hline$\$ 201$ & 0.01009 & 24.08 & $\mathbf{3 1 . 3 8}$ & 124 \\
\hline 0168 & 0.01004 & 34.80 & 46.41 & 1.46 \\
\hline 0065 & 0.01081 & 5025 & 5120 & 1.53 \\
\hline$\pi 07$ & 0.01080 & 43.42 & 68.65 & 1.61 \\
\hline 8063 & 0.01077 & 50.98 & 68.52 & 1.60 \\
\hline 12470 & 0.01087 & 70.46 & 91.83 & 1.87 \\
\hline 16002 & 0.01114 & 00.05 & 118.67 & 1.87 \\
\hline 20050 & 0.01100 & 118.48 & 165.60 & 1.82 \\
\hline 28435 & 0.01133 & 164.04 & 214.03 & 1.91 \\
\hline 36769 & 0.01123 & 21122 & 275.58 & 1.87 \\
\hline 48274 & 0.01128 & 277.84 & 302.50 & 1.00 \\
\hline 65620 & 0.01137 & 367.72 & 470.78 & 1.80 \\
\hline 83950 & 0.01160 & 400.03 & 639.35 & 1.80 \\
\hline 110777 & 0.01214 & 681.44 & 663.00 & 1.87 \\
\hline
\end{tabular}

Friction $(1): p / e=3.92, \theta / D=0.2, \downarrow_{A}=9.276, \downarrow_{t}=0.0169$

\begin{tabular}{|c|c|c|c|c|c|c|c|}
\hline Re & 1 & $R_{m}$ & $t_{m}$ & Ro & 1 & $\boldsymbol{R o}_{m}$ & $f_{m}$ \\
\hline 148 & 0.64497 & 1370 & 0.01000 & 3047 & 0.77515 & 20284 & 0.01310 \\
\hline 182 & 0.51775 & 1781 & 0.00875 & 3952 & 0.74704 & 36655 & 0.01263 \\
\hline 226 & 0.47337 & 2100 & 0.00800 & 5125 & 0.72041 & 47538 & 0.01218 \\
\hline 204 & 0.53846 & 2723 & 0.00910 & 5837 & 0.72041 & 54137 & 0.01218 \\
\hline 403 & 0.74704 & 3734 & 0.01283 & 7150 & 0.69379 & 66405 & 0.01173 \\
\hline 522 & 0.89645 & 4843 & 0.01515 & 8817 & 0.69379 & 91054 & 0.01173 \\
\hline 600 & 1.00000 & 6398 & 0.01690 & 12497 & 0.69370 & 115014 & 0.01173 \\
\hline 800 & 1.00000 & 7423 & 0.01690 & 14770 & 0.72041 & 130998 & 0.01218 \\
\hline 878 & 1.00000 & 8146 & 0.01690 & 18117 & 0.74704 & 168045 & 0.01263 \\
\hline 1000 & 1.00000 & 8270 & 0.01600 & 23400 & 0.74704 & 217128 & 0.01263 \\
\hline 1450 & 0.84911 & 13448 & 0.01435 & 28820 & 0.77515 & 267324 & 0.01310 \\
\hline 1846 & 0.86391 & 17120 & 0.01460 & 32821 & 0.77515 & 304432 & 0.01310 \\
\hline 2306 & 0.84011 & 21393 & 0.01435 & & & & \\
\hline
\end{tabular}


Stanton number ratios ():

\begin{tabular}{|c|c|c|c|c|}
\hline $\mathbf{R e}_{\mathrm{m}}$ & $f_{m}$ & $\varphi^{+}$ & $\theta_{m}^{+}$ & syst \\
\hline 4124 & 0.01570 & 68.60 & 6828 & 121 \\
\hline 8467 & 0.01810 & 81.34 & $\bullet 8.10$ & 1.10 \\
\hline 6875 & 0.01606 & 104.89 & 126.62 & 1.25 \\
\hline 870 & 0.01604 & 13428 & 161.24 & 1.48 \\
\hline 11458 & 0.01567 & 167.87 & 202.4 & 1.72 \\
\hline 14100 & 0.01419 & 186.97 & 237.54 & 2.15 \\
\hline 18085 & 0.01458 & 282.86 & 341.24 & 2.65 \\
\hline 24212 & 0.01380 & 333.56 & 40226 & 287 \\
\hline 34924 & 0.01268 & 461.14 & 556.13 & 3.11 \\
\hline 43056 & 0.01232 & 560.53 & 675.89 & 3.41 \\
\hline 52163 & 0.01218 & 675.01 & 814.05 & 3.25 \\
\hline 72155 & 0.01160 & 911.32 & 1009.04 & 3.42 \\
\hline 82760 & 0.01173 & 1178.28 & 1420.99 & 3.38 \\
\hline 116371 & 0.01173 & 1477.84 & 1782.38 & 3.42 \\
\hline 131485 & 0.01204 & 1691.60 & 2040.06 & 3.56 \\
\hline 176874 & 0.01265 & 2332.89 & 2813.56 & 3.21 \\
\hline 200941 & 0.01261 & 2725.23 & 3286.59 & 3.42 \\
\hline 202314 & 0.01305 & 351428 & 4238.18 & 3.60 \\
\hline
\end{tabular}

Friction (k): $p / e=9.8, e / D=0.2, \downarrow_{A}=8.616, \downarrow_{f}=0.0277$

\begin{tabular}{|c|c|c|c|c|c|c|c|}
\hline Ro & $f$ & $\mathbf{R o}_{m}$ & $t_{m}$ & Ro & 1 & $\mathbf{R}_{m}$ & $t_{m}$ \\
\hline 113 & 0.42226 & 970 & 0.01170 & 1896 & 0.88787 & 16338 & 0.02460 \\
\hline 147 & 0.37110 & 1264 & 0.01028 & 2041 & 0.85845 & 17586 & 0.02378 \\
\hline 167 & 0.32249 & 1442 & 0.00803 & 2210 & 0.86490 & 19030 & 0.02396 \\
\hline 204 & 0.33291 & 1757 & 0.00522 & 2007 & 0.84253 & 20653 & 0.02334 \\
\hline 230 & 0.29312 & 1882 & 0.00812 & 2585 & 0.85204 & 22274 & 0.02361 \\
\hline 244 & 0.28876 & 2100 & 0.00600 & 2949 & 0.84886 & 25410 & 0.02352 \\
\hline 254 & 0.29588 & 2187 & 0.00820 & 3306 & 0.85684 & 28486 & 0.02374 \\
\hline 202 & 0.31888 & 2256 & 0.00883 & 3913 & 0.86167 & 33715 & 0.02387 \\
\hline 278 & 0.32370 & 2001 & 0.00097 & 4413 & 0.87631 & 38018 & 0.02428 \\
\hline 292 & 0.40296 & 2515 & 0.01116 & 4928 & 0.86977 & 42456 & 0.02410 \\
\hline
\end{tabular}




\begin{tabular}{|c|c|c|c|c|c|c|c|}
\hline Ro & 1 & $\mathbf{m}_{m}$ & $t_{m}$ & Po & 1 & $R_{m}$ & $t_{m}$ \\
\hline so7 & 0.00284 & 2845 & 0.01670 & 5004 & 0.87467 & 45009 & 0.02423 \\
\hline 328 & 0.00758 & 2025 & 0.01982 & 8020 & 0.88787 & 55670 & 0.02460 \\
\hline 340 & 0.73005 & 2031 & 0.02047 & 7024 & 0.88124 & $6051 \theta$ & 0.02441 \\
\hline 394 & 0.00551 & $\sec 5$ & 0.00202 & 8378 & 0.91315 & 72185 & 0.00530 \\
\hline 423 & 0.03468 & 8848 & 0.00312 & 9338 & 0.02215 & 80456 & 0.02582 \\
\hline 475 & 0.83400 & 4000 & $0.00 \times 98$ & 11117 & 0.92174 & 85780 & 0.02554 \\
\hline 600 & 0.85045 & 4738 & 0.02358 & 11874 & 0.91820 & 100301 & 0.02544 \\
\hline 846 & 0.85045 & 6054 & 0.02358 & 13578 & 0.84444 & 117840 & 0.02617 \\
\hline 747 & 0.87631 & enss & 0.02428 & 16505 & 0.02174 & 142189 & 0.02554 \\
\hline 852 & 0.87785 & 7341 & 0.02432 & 20385 & 0.94444 & 175629 & 0.02617 \\
\hline 883 & 0.87467 & 8472 & 0.02423 & 23527 & 0.97864 & 202008 & 0.02711 \\
\hline 1085 & 0.87467 & $\cos 5$ & 0.02423 & 27846 & 0.90527 & 239906 & 0.02757 \\
\hline 117 & 0.87631 & 10145 & 0.02428 & 31338 & 0.89713 & 200997 & 0.02763 \\
\hline 1275 & 0.86977 & 10083 & 0.02410 & 35681 & 1.03130 & 307415 & 0.02857 \\
\hline 1383 & 0.87950 & 11914 & 0.02437 & 42149 & 1.04491 & 353141 & 0.02895 \\
\hline 1571 & 0.87631 & 13530 & 0.02428 & 48835 & 1.07466 & 420738 & 0.02977 \\
\hline 1718 & 0.85652 & 14801 & 0.02401 & & & & \\
\hline
\end{tabular}

Friction $(n): p / e=26, e / D=0.25, \downarrow_{R}=11.589, \downarrow_{f}=0.0138$

\begin{tabular}{|c|c|c|c|c|c|c|c|}
\hline Re & 1 & $\boldsymbol{R}_{\mathrm{m}}$ & $t_{m}$ & Ro & 1 & $\mathbf{R}_{\boldsymbol{e}_{\mathrm{m}}}$ & $t_{m}$ \\
\hline 114 & 0.71845 & 1320 & 0.00995 & 2937 & 0.00660 & 34034 & 0.01240 \\
\hline 145 & 0.00414 & 1681 & 0.00960 & 4102 & 0.91287 & 47530 & 0.01263 \\
\hline 165 & 0.60015 & 1912 & 0.00830 & 6838 & 0.91287 & 67651 & 0.01263 \\
\hline 181 & 0.57845 & 2100 & 0.00800 & 6648 & 0.82014 & 77040 & 0.01285 \\
\hline 218 & 0.65884 & 2020 & 0.00025 & 9460 & 0.80014 & 100637 & 0.01285 \\
\hline 204 & 0.80441 & 3408 & 0.01113 & 10180 & 0.82014 & 118088 & 0.01285 \\
\hline 375 & 0.80441 & 4347 & 0.01113 & 11434 & 0.92914 & 132508 & 0.01285 \\
\hline 485 & 0.81887 & 5602 & 0.04133 & 14497 & 0.91287 & 168000 & 0.01263 \\
\hline 608 & 0.81887 & 7003 & 0.01133 & 18121 & 0.02014 & 210000 & 0.01285 \\
\hline $8 \infty$ & 0.80441 & 8092 & 0.01113 & 22644 & 0.82914 & 202420 & 0.01285 \\
\hline 1038 & $0.80 \times 41$ & 12052 & 0.01113 & 26271 & 0.81287 & 304452 & 0.01263 \\
\hline 1397 & 0.84780 & 16194 & 0.01173 & 29917 & 0.91287 & 346705 & 0.01263 \\
\hline
\end{tabular}




\begin{tabular}{|c|c|c|c|c|c|c|c|}
\hline Po & 1 & $\boldsymbol{n}_{\boldsymbol{m}}$ & tm & Po & 1 & $\mathbf{P}_{m}$ & $t_{m}$ \\
\hline 1918 & 0.84780 & 22109 & 0.01178 & 50224 & 0.50814 & 573448 & 0.01285 \\
\hline 2027 & 0.80000 & 50441 & 0.01240 & & & & \\
\hline
\end{tabular}

Stanton number ratios (n):

\begin{tabular}{|c|c|c|c|c|}
\hline $\mathbf{P o}_{\mathrm{m}}$ & $t_{m}$ & $0^{+}$ & $a_{m}^{+}$ & syst \\
\hline 5727 & 0.01134 & 70.12 & 107.82 & 1.12 \\
\hline 8010 & 0.01124 & 110.18 & 150.15 & 1.17 \\
\hline 10005 & 0.01110 & 150.24 & 204.75 & 1.34 \\
\hline 14291 & 0.01144 & 188.25 & 270.17 & 1.60 \\
\hline 18892 & 0.01180 & 266.22 & 302.81 & 1.84 \\
\hline 25413 & 0.01194 & 300.18 & 490.88 & 2.36 \\
\hline 30257 & 0.01230 & 436.91 & 585.42 & 2.54 \\
\hline 45183 & 0.01258 & 657.46 & 805.88 & 2.85 \\
\hline 57694 & 0.01263 & 840.98 & 1146.09 & 2.85 \\
\hline 73653 & 0.01276 & 1079.34 & 1470.93 & 2.96 \\
\hline 94027 & 0.01295 & 1388.00 & 1891.58 & 3.01 \\
\hline 100245 & 0.01272 & 2812.30 & 3832.64 & 3.02 \\
\hline 277298 & 0.01277 & 4054.72 & 5539.44 & 3.02 \\
\hline 318825 & 0.01258 & 4639.40 & 6022.62 & 2.80 \\
\hline
\end{tabular}

(c) Transverse ring inserts with clearance for flow near the wall

Friction $(e=2.25 \mathrm{~mm}): p / e=9.8, e / D=0.045, \nabla_{A}=1.795, \downarrow_{1}=0.254$

\begin{tabular}{|c|c|c|c|c|c|c|c|}
\hline$R_{0}$ & $f$ & $R_{0_{m}}$ & $t_{m}$ & $R_{0}$ & $f$ & $R_{\theta_{m}}$ & $f_{m}$ \\
\hline 453 & 0.06112 & 813 & 0.01550 & 5266 & 0.08251 & 9453 & 0.02093 \\
\hline 569 & 0.05363 & 1022 & 0.01360 & 6730 & 0.08251 & 12081 & 0.02093 \\
\hline 716 & 0.04535 & 1285 & 0.01150 & 8325 & 0.08123 & 14944 & 0.02060 \\
\hline 900 & 0.03845 & 1616 & 0.00975 & 10467 & 0.07857 & 18788 & 0.01993 \\
\hline 993 & 0.03431 & 1783 & 0.00870 & 13598 & 0.07857 & 24408 & 0.01893 \\
\hline 1170 & 0.03155 & 2100 & 0.00800 & 14517 & 0.07857 & 26058 & 0.01993 \\
\hline 1249 & 0.04535 & 2242 & 0.01150 & 20804 & 0.07729 & 37343 & 0.01860 \\
\hline 1378 & 0.05989 & 2473 & 0.01773 & 26157 & 0.08350 & 46951 & 0.02118 \\
\hline
\end{tabular}




\begin{tabular}{|c|c|c|c|c|c|c|c|}
\hline Bo & 1 & $\mathbf{R}_{m}$ & $t_{m}$ & Ao & 1 & $\boldsymbol{n o}_{\mathrm{m}}$ & $t_{m}$ \\
\hline 1471 & 0.07857 & 2040 & 0.01893 & 35880 & 0.08251 & 60004 & 0.02023 \\
\hline 1010 & 0.00424 & 3420 & 0.02000 & 41348 & 0.08123 & 74220 & 0.02060 \\
\hline 2482 & 0.00119 & 455 & 0.02313 & 53715 & 0.08251 & 06419 & 0.02093 \\
\hline 3025 & 0.00110 & 6788 & 0.02313 & 67537 & 0.08380 & 121228 & 0.02128 \\
\hline 4054 & 0.02537 & 727 & 0.02165 & & & & \\
\hline
\end{tabular}

Heat transfer $(e=2.25 \mathrm{~mm}): \downarrow_{A}=2.516$

\begin{tabular}{|c|c|c|c|c|c|c|c|}
\hline Ro & Nu & $\mathbf{P}_{\mathrm{m}}$ & $\mathrm{Nu}_{\mathrm{m}}$ & Ro & $\mathrm{Nu}$ & $\mathbf{P o}_{\mathrm{m}}$ & $N u_{m}$ \\
\hline 603 & 7.5 & 1288 & 7.6 & 6730 & 61.8 & 10958 & 51.8 \\
\hline 733 & 10.7 & 1844 & 10.7 & 2211 & 64.1 & 23179 & 64.1 \\
\hline 1086 & 13.9 & 2732 & 13.8 & 12500 & 82.1 & 31682 & 82.1 \\
\hline 1509 & 18.4 & 3797 & 18.4 & 15337 & 80.6 & 38594 & 80.6 \\
\hline 2202 & 24.7 & 6542 & 24.7 & 21311 & 117.5 & 53026 & 147.5 \\
\hline 2683 & 28.7 & 6752 & 28.7 & 26829 & 152.2 & 67513 & 152.2 \\
\hline 3162 & 31.1 & 7958 & 31.1 & 41145 & 184.8 & 103537 & 184.8 \\
\hline 4850 & 40.5 & 12204 & 40.5 & 58119 & 247.1 & 140250 & 247.1 \\
\hline
\end{tabular}

Stanton number ratios $(\theta=2.25 \mathrm{~mm})$

\begin{tabular}{|c|c|c|c|c|}
\hline $\mathbf{P}_{\boldsymbol{e}_{\mathrm{m}}}$ & $t_{m}$ & $0^{+}$ & $\bullet_{m}^{+}$ & stst \\
\hline 803 & 0.01468 & 3.85 & 3.48 & 1.02 \\
\hline 1315 & 0.01131 & 4.92 & 4.45 & 1.07 \\
\hline 1849 & 0.00737 & 5.89 & 5.32 & 1.22 \\
\hline 2708 & 0.02054 & 13.66 & 12.35 & 1.40 \\
\hline 3053 & 0.02379 & 21.47 & 19.40 & 1.83 \\
\hline 4816 & 0.00205 & 25.60 & 23.22 & 2.00 \\
\hline 5576 & 0.02314 & 30.40 & 27.47 & 2.08 \\
\hline 8705 & 0.02004 & 44.35 & 40.00 & 2.15 \\
\hline 12006 & 0.00002 & 61.60 & 55.68 & 2.15 \\
\hline 16533 & 0.02050 & 02.82 & 74.85 & 2.15 \\
\hline 20598 & 0.01887 & 112.13 & 101.35 & 2.12 \\
\hline 27520 & 0.01887 & 136.63 & 123.49 & 2.07 \\
\hline 38250 & 0.01969 & 188.97 & 170.80 & 2.03 \\
\hline
\end{tabular}




\begin{tabular}{|c|c|c|c|c|}
\hline $\mathrm{R}_{\mathrm{m}}$ & s & $e^{+}$ & $a_{m}^{+}$ & $84 / 54$ \\
\hline 48155 & 0.02130 & 247.42 & 203.62 & 223 \\
\hline 73850 & 0.00000 & 573.17 & 33728 & 1.01 \\
\hline 104316 & 0.02100 & 523.40 & 482.00 & 201 \\
\hline
\end{tabular}

Friction $(\theta=3.75 \mathrm{~mm}): p / \theta=9.8, \theta / D=0.075, \downarrow_{R}=2.992, \downarrow_{1}=0.113$

\begin{tabular}{|c|c|c|c|c|c|c|c|}
\hline Ro & 1 & $\mathbf{R} \boldsymbol{o}_{\mathrm{m}}$ & $t_{m}$ & Aro & 1 & $\mathbf{R}_{\mathrm{m}}$ & $t_{m}$ \\
\hline 304 & 0.13556 & $\infty$ & 0.01525 & 5532 & 0.13333 & 16551 & 0.01500 \\
\hline 300 & 0.11311 & 1100 & 0.01273 & 6850 & 0.13111 & 20496 & 0.01475 \\
\hline 485 & 0.00078 & 1450 & 0.01010 & 0912 & 0.13111 & 20566 & 0.01475 \\
\hline 579 & 0.07467 & 1731 & 0.00840 & 10560 & 0.13111 & 31504 & 0.01475 \\
\hline 702 & 0.07111 & 2100 & 0.00800 & 13010 & 0.13111 & 38827 & 0.01475 \\
\hline 851 & 0.10067 & 2547 & 0.01133 & 15336 & 0.13333 & 45886 & 0.01500 \\
\hline 1033 & 0.12280 & 3002 & 0.01383 & 18307 & 0.13556 & 57767 & 0.01525 \\
\hline 1218 & 0.13778 & 3645 & 0.01550 & 22758 & 0.13778 & 68093 & 0.01550 \\
\hline 1534 & 0.15000 & 4589 & 0.01688 & 27724 & 0.14000 & 82950 & 0.01575 \\
\hline 1808 & 0.15000 & 5400 & 0.01688 & 33773 & 0.14000 & 101049 & 0.01575 \\
\hline 2202 & 0.14756 & 6589 & 0.01660 & 40471 & 0.14000 & 121089 & 0.01575 \\
\hline 2596 & 0.14511 & 7787 & 0.01633 & 53527 & 0.14511 & 160152 & 0.01633 \\
\hline 3162 & 0.13556 & 9462 & 0.01525 & 65206 & 0.14756 & 195095 & 0.01660 \\
\hline 3852 & 0.13111 & 11526 & 0.01475 & & & & \\
\hline
\end{tabular}

Heat transfer $(e=3.75 \mathrm{~mm}): \downarrow_{A}=4.194$

\begin{tabular}{|c|c|c|c|c|c|c|c|}
\hline Ro & $\mathrm{Nu}$ & $\mathbf{R}_{m}$ & $N u_{m}$ & Ro & $\mathrm{Nu}$ & $\mathbf{R}_{\mathrm{m}}$ & $N u_{m}$ \\
\hline 441 & 6.6 & 1851 & 6.6 & 6852 & 57.2 & 28735 & 57.2 \\
\hline 632 & 8.2 & 2653 & 0.2 & 7688 & 59.1 & 32242 & 50.1 \\
\hline 800 & 11.4 & 3301 & 11.4 & 8830 & 75.6 & 41263 & 75.6 \\
\hline 1034 & 13.7 & 4335 & 13.7 & 12184 & 80.6 & 51100 & 80.6 \\
\hline 1345 & 17.5 & 5840 & 17.5 & 14842 & 101.6 & 62248 & 101.6 \\
\hline 1749 & 20.6 & 7337 & 20.6 & 18081 & 122.1 & 75831 & 122.1 \\
\hline 2276 & 26.8 & 8546 & 26.8 & 22763 & 144.2 & 95465 & 144.2 \\
\hline 2773 & 30.6 & 11650 & 50.6 & 28189 & 170.4 & 118224 & 170.4 \\
\hline
\end{tabular}




\begin{tabular}{|c|c|c|c|c|c|c|c|}
\hline Ao & Nu & $n_{m}$ & $\mathrm{Nu}_{\mathrm{m}}$ & Ro & Nus & $\mathbf{R}_{\mathrm{m}}$ & $\mathrm{Nu}_{\mathrm{m}}$ \\
\hline 3060 & 827 & 12836 & 227 & 80077 & 208 & 151304 & 200 \\
\hline 3750 & 20.5 & 15806 & $\mathbf{8 3 . 5}$ & 40948 & 237.6 & 184316 & 237.6 \\
\hline 4004 & 44.7 & 10005 & 44.7 & 53537 & 280.7 & 224531 & 200.7 \\
\hline 8000 & 62.7 & 24782 & 52.7 & & & & \\
\hline
\end{tabular}

Stanton number ratios $(\theta=3.75 \mathrm{~mm})$ :

\begin{tabular}{|c|c|c|c|c|}
\hline $\mathbf{R}_{m}$ & $t_{m}$ & $e^{+}$ & $\bullet_{m}^{+}$ & Exst \\
\hline 1320 & 0.01005 & 7.30 & 7.32 & 1.03 \\
\hline 1692 & 0.00784 & 8.85 & 8.88 & 1.08 \\
\hline 2419 & 0.01028 & 12.96 & 13.00 & 1.16 \\
\hline 3082 & 0.01383 & 10.21 & 18.28 & 1.22 \\
\hline 4023 & 0.01620 & 27.14 & 27.23 & 1.45 \\
\hline 5233 & 0.01691 & 35.97 & 36.09 & 1.65 \\
\hline 6809 & 0.01654 & 46.28 & 46.44 & 2.08 \\
\hline 8205 & 0.01633 & 56,02 & 56.21 & 2.14 \\
\hline 9155 & 0.01637 & 81.80 & 62.11 & 2.23 \\
\hline 11338 & 0.01535 & 74.25 & 74.50 & 2.40 \\
\hline 14041 & 0.01475 & 80.13 & $\infty .44$ & 2.46 \\
\hline 17676 & 0.01500 & 114.42 & 114.82 & 2.50 \\
\hline 20406 & 0.01475 & 131.56 & 132.01 & 2.34 \\
\hline 22097 & 0.01467 & 147.10 & 147.70 & 2.26 \\
\hline 20432 & 0.01477 & 189.05 & 189.70 & 2.41 \\
\hline 36448 & 0.01472 & 233.73 & 234.54 & 2.43 \\
\hline 44400 & 0.01494 & 286.65 & 2837.84 & 2.39 \\
\hline 51320 & 0.01510 & 352.28 & 353.50 & 2.16 \\
\hline 68093 & 0.01550 & 448.05 & 440.50 & 240 \\
\hline 84326 & 0.01576 & 550.47 & 561.40 & 2.41 \\
\hline 107921 & 0.01575 & 715.75 & 718.22 & 2.30 \\
\hline 131468 & 0.01575 & 871.90 & 875.00 & 2.35 \\
\hline 160152 & 0.01682 & 1081.46 & 1085.10 & 2.41 \\
\hline
\end{tabular}


(d) Koch (1960): Enhancement factors

\begin{tabular}{|c|c|c|c|c|c|}
\hline$\infty$ & Mo & $\phi_{R}$ & $t_{1}$ & $4^{+}$ & $\theta_{n, \max }$ \\
\hline 0.1 & $\infty$ & 3.941 & 0.217 & 225 & 2.01 \\
\hline 0.1 & 0.8 & 3.000 & 0.219 & 6.81 & 288 \\
\hline 0.2 & 9.8 & 12.088 & 0.028 & 4.86 & 4.00 \\
\hline 0.1 & 3.02 & 2.800 & 0.175 & 4.66 & 2.61 \\
\hline 0.045 & 9.8 & 2.516 & 0.255 & 4.18 & 2.33 \\
\hline 0.075 & 0.8 & 4.184 & 0.113 & 297 & 258 \\
\hline \multicolumn{2}{|c|}{ emooth } & 1 & 1 & 1 & 1 \\
\hline
\end{tabular}


Table A.8

Nunner (1956)

Friction and heat transfer data for smooth and rough tubes

(a) Smooth tube

Friction (smooth): $D=50.06 \mathrm{~mm}, \downarrow_{R}=0.837, \downarrow_{1}=0.970$

\begin{tabular}{|c|c|c|c|c|c|c|c|}
\hline Ro & 1 & $\mathbf{R}_{m}$ & $f_{m}$ & Po & 1 & $R_{\boldsymbol{o}_{m}}$ & $f_{m}$ \\
\hline 928 & 0.01746 & 778 & $0.016 \infty 3$ & 6292 & 0.00927 & 5264 & 0.00899 \\
\hline 865 & 0.01681 & 808 & 0.01630 & 6583 & 0.00014 & 5508 & 0.00886 \\
\hline 1004 & 0.01617 & 840 & 0.01568 & 6977 & 0.00904 & 5837 & 0.00877 \\
\hline 1038 & 0.01563 & 868 & 0.01516 & 7419 & 0.00891 & 6207 & 0.00864 \\
\hline 1076 & 0.01514 & $\infty 00$ & 0.01468 & 7836 & 0.00877 & 6556 & 0.00850 \\
\hline 1124 & 0.01462 & 940 & 0.01417 & 8340 & 0.00863 & 6978 & 0.00836 \\
\hline 1173 & 0.01414 & 881 & 0.01371 & 8824 & 0.00852 & 7382 & 0.00826 \\
\hline 1216 & 0.01381 & 1017 & 0.01339 & 9430 & 0.00838 & 7898 & 0.00813 \\
\hline 1262 & 0.01343 & 1056 & 0.01302 & 10116 & 0.00825 & 8463 & 0.00800 \\
\hline 1303 & 0.01317 & 1000 & 0.01277 & 10730 & 0.00811 & 8977 & 0.00787 \\
\hline 1344 & 0.01284 & 1125 & 0.01246 & 11498 & 0.00797 & 9020 & 0.00773 \\
\hline 1389 & 0.01254 & 1162 & 0.01216 & 12332 & 0.00786 & 10318 & 0.00762 \\
\hline 1443 & 0.01217 & 1207 & 0.01181 & 13081 & 0.00774 & 10944 & 0.00751 \\
\hline 1510 & 0.01180 & 1263 & 0.0114 & 13887 & 0.00763 & 11619 & 0.00740 \\
\hline 1588 & 0.01143 & 1320 & 0.01100 & 14667 & 0.00753 & 12271 & 0.00731 \\
\hline 1654 & 0.01102 & 1384 & 0.01069 & 15557 & 0.00740 & 13016 & 0.00717 \\
\hline 1731 & 0.01068 & 1448 & 0.01036 & 16515 & 0.00730 & 13818 & 0.00708 \\
\hline 1785 & 0.01030 & 1494 & 0.01008 & 17488 & 0.00715 & 14632 & 0.00693 \\
\hline 1861 & 0.01012 & 1557 & 0.00082 & 18392 & 0.00708 & 15388 & 0.00687 \\
\hline 1831 & 0.00988 & 1616 & 0.00558 & 18276 & 0.00700 & 16128 & 0.00681 \\
\hline 2000 & 0.00956 & $16: 8$ & 0.00827 & 20490 & 0.00591 & 17151 & 0.00671 \\
\hline 2121 & 0.00923 & 1775 & 0.00895 & 21688 & 0.00684 & 18145 & 0.00663 \\
\hline 2218 & 0.00502 & 1855 & 0.00874 & 23480 & 0.00665 & 19645 & 0.00645 \\
\hline 2316 & 0.00879 & 1838 & 0.00852 & 25313 & 0.00655 & 21179 & 0.00636 \\
\hline 2399 & 0.00866 & 2007 & 0.00830 & 27057 & 0.00644 & 22638 & 0.00524 \\
\hline 2476 & 0.00860 & 2072 & 0.00834 & 28773 & 0.00635 & 24073 & 0.00516 \\
\hline
\end{tabular}




\begin{tabular}{|c|c|c|c|c|c|c|c|}
\hline Po & 1 & $\boldsymbol{R}_{\mathrm{m}}$ & $t_{m}$ & $\mathbf{R}_{0}$ & $f$ & $\boldsymbol{R}_{\boldsymbol{m}}$ & $\mathbf{t}_{m}$ \\
\hline 2547 & 0.00865 & 2131 & 0.00030 & $\operatorname{sos} 66$ & 0.00524 & 25008 & 0.00505 \\
\hline 2815 & 0.00876 & 2188 & 0.00849 & 35240 & 0.00612 & 27811 & 0.00593 \\
\hline 2708 . & 0.00084 & 2266 & 0.00667 & 35835 & 0.00600 & 20082 & 0.00582 \\
\hline 2741 & 0.00915 & 2003 & 0.00887 & 38434 & 0.00500 & 32157 & 0.00572 \\
\hline 2836 & 0.00053 & 2073 & 0.00024 & 40977 & 0.00581 & 34284 & 0.00564 \\
\hline 2030 & 0.00081 & 2451 & 0.00051 & 43270 & 0.00572 & 36210 & 0.00554 \\
\hline 3008 & 0.01000 & 2517 & 0.00978 & 46142 & 0.00563 & 38606 & 0.00546 \\
\hline 3009 & 0.01034 & 2584 & 0.01003 & 49786 & 0.00554 & 41654 & 0.00537 \\
\hline 3235 & 0.01052 & 2708 & 0.01020 & $5 \operatorname{sos} 4$ & 0.00545 & 44372 & 0.00520 \\
\hline 3567 & 0.01002 & 2817 & 0.01080 & 55918 & 0.00535 & 46785 & 0.00519 \\
\hline 3446 & 0.01050 & 2883 & 0.01027 & 50617 & 0.00527 & 49880 & 0.00511 \\
\hline 3556 & 0.01048 & 2975 & 0.01016 & 61847 & 0.00524 & 51745 & 0.00508 \\
\hline 3730 & 0.01037 & 3121 & 0.01006 & 60002 & 0.00514 & 55975 & 0.00498 \\
\hline 3917 & 0.01024 & 327 & 0.00993 & 70963 & 0.00506 & 50373 & 0.00490 \\
\hline 4147 & 0.01017 & 3470 & 0.00986 & 74249 & 0.00501 & 62122 & 0.00486 \\
\hline 4391 & 0.01010 & 3674 & 0.00979 & 78622 & 0.00495 & 65781 & 0.00480 \\
\hline 4500 & 0.00997 & 3848 & 0.00867 & 81772 & 0.00488 & 68416 & 0.00473 \\
\hline 4849 & 0.00088 & 4057 & 0.00958 & 85412 & 0.00482 & 71462 & 0.00467 \\
\hline 5065 & 0.00976 & 4237 & 0.00946 & 91002 & 0.00476 & 76189 & 0.00462 \\
\hline 5437 & 0.00059 & 4549 & 0.00930 & 93906 & 0.00474 & 78635 & 0.00460 \\
\hline 5718 & 0.00042 & 4784 & 0.00013 & & & & \\
\hline
\end{tabular}

Heat transfer (smooth):

\begin{tabular}{|c|c|c|c|c|c|c|c|}
\hline Po & $N u_{m}$ & Ro & $\mathrm{Nu}_{\mathrm{m}}$ & Ro & $N u_{m}$ & Ro & $\mathrm{Nu}_{\mathrm{m}}$ \\
\hline 552 & 7.32 & 3763 & 14.92 & 12311 & 35.82 & 36548 & 84.61 \\
\hline 584 & 7.64 & 8057 & 15.28 & 12810 & 36.80 & 39096 & 88.50 \\
\hline 635 & 8.18 & 4128 & 15.56 & 1337 & 38.18 & 40000 & 91.88 \\
\hline 679 & 8.63 & 4343 & 16.04 & 14117 & 39.80 & 43230 & 85.77 \\
\hline 750 & 9.15 & 4515 & 16.50 & 14868 & 41.41 & 45343 & 10021 \\
\hline 815 & 9.50 & 4776 & 17.10 & 15781 & 43.46 & 48642 & 105.38 \\
\hline 893 & 10.04 & 5055 & 17.81 & 16869 & 45.83 & 51010 & 109.41 \\
\hline 074 & 10.40 & 5312 & 18.53 & 17478 & 47.28 & 53911 & 115.05 \\
\hline 1050 & 10.79 & 5551 & 10.35 & 17993 & 48.16 & 50938 & 120.29 \\
\hline
\end{tabular}




\begin{tabular}{|c|c|c|c|c|c|c|c|}
\hline $\mathbf{R}$ & $\mathrm{Nu}_{\mathrm{m}}$ & Po & $\mathbf{N u}_{m}$ & $\boldsymbol{R}$ & $\mathrm{Nu}_{\mathrm{m}}$ & Ro & $N u_{m}$ \\
\hline 1128 & 11.08 & 6e21 & 20.13 & 19003 & 60.53 & 60024 & 124.72 \\
\hline 1203 & 1125 & 8050 & 20.63 & 10057 & 52.43 & 60108 & 129.67 \\
\hline 1333 & 11.41 & 6158 & 21.15 & 20721 & 54.20 & 05570 & 134.53 \\
\hline 1441 & 11.51 & 6408 & 21.88 & 21167 & 50.25 & 68082 & 138.01 \\
\hline 1578 & 11.84 & evre & 22.88 & 21792 & 68.71 & 70340 & 141.67 \\
\hline 1674 & 11.72 & 7135 & 23.74 & 22787 & $\mathbf{E 0 . 5 1}$ & 73308 & 146.98 \\
\hline 1843 & 11.86 & 70.81 & 24.37 & 20477 & 0.02 & 76481 & 151.85 \\
\hline 1080 & 11.03 & 7008 & 20.07 & 24324 & 61.65 & 78738 & 155.77 \\
\hline 2147 & 12.14 & 8124 & 25.84 & 25140 & 68.07 & 81523 & 150.56 \\
\hline 2005 & 12.36 & 0620 & $\mathbf{2 6 . 8 7}$ & 25928 & 65.08 & 84886 & 184.50 \\
\hline 2510 & 12.66 & 0003 & 27.80 & 26655 & 68.32 & 87515 & 168.15 \\
\hline 2760 & 13.15 & $\operatorname{ses} \theta$ & 28.88 & 27853 & 68.66 & 80354 & 172.49 \\
\hline 2076 & 13.45 & ヘ874 & 30.01 & 20334 & 71.84 & 82757 & 175.57 \\
\hline 3127 & 13.75 & 100667 & 31.24 & 30587 & 74.12 & 86790 & 180.87 \\
\hline 3284 & 13.87 & 10782 & 32.39 & 32236 & 77.61 & 80042 & 184.10 \\
\hline 3444 & 14.32 & 11243 & 33.61 & 34605 & 81.55 & 83731 & 184.75 \\
\hline 5000 & 14.58 & 11840 & 34.87 & & & & \\
\hline
\end{tabular}

(b) Rough tube deta

(m) denotes extracted value of $R e$ or $f$ based on smooth tube mean diameter $d_{m}$ (i) denotes recomputed value of $R \theta$ or $f$ based on maximum internal tube diameter $D$

Friction (A20, data set (4)): rectangular, $p / e=20, e / D=0.04, d_{m}=49.82 \mathrm{~mm}$ $\downarrow_{R}(m)=1.053, \downarrow_{A}(m)=0.615, \downarrow_{R}(D)=1.056, \downarrow_{1}(D)=0.612$

\begin{tabular}{|c|c|c|c|c|c|}
\hline$R_{\theta}(m)$ & $f(m)$ & Po 0 & 10 & $\mathbf{R}_{\boldsymbol{m}}$ & $t_{m}$ \\
\hline 993 & 0.02178 & 988 & 0.02189 & 1044 & 0.01330 \\
\hline 1028 & 0.02068 & 1002 & 0.02076 & 1081 & 0.01270 \\
\hline 1069 & 0.01804 & 1084 & 0.02003 & 1124 & 0.01226 \\
\hline 1120 & 0.01914 & 1114 & 0.01823 & 1177 & 0.01177 \\
\hline 1154 & 0.01847 & 1148 & 0.01858 & 1213 & 0.01138 \\
\hline 1192 & 0.01778 & 1186 & 0.01785 & 1253 & 0.01002 \\
\hline 1228 & 0.01717 & 1220 & 0.01725 & 1280 & 0.01055 \\
\hline 1280 & 0.01655 & 1283 & 0.01663 & 1355 & 0.01017 \\
\hline
\end{tabular}




\begin{tabular}{|c|c|c|c|c|c|}
\hline Po (m) & $f(m)$ & $P 0$ & 10 & $\mathbf{R o}_{\mathrm{m}}$ & $f_{m}$ \\
\hline 1850 & $0.01=03$ & 1320 & 0.01601 & 1308 & 0.00079 \\
\hline 1973 & 0.01547 & 1857 & 0.01555 & 1444 & 0.00851 \\
\hline 1458 & 0.01475 & 1440 & 0.01480 & 1650 & 0.00908 \\
\hline 1502 & 0.01450 & 1485 & 0.01437 & 1570 & 0.00879 \\
\hline 1673 & 0.01390 & 1588 & 0.01376 & 1864 & 0.00842 \\
\hline 1023 & 0.01330 & 1626 & 0.01340 & 1717 & 0.00820 \\
\hline 1727 & 0.01313 & 1718 & 0.01319 & 1815 & 0.00807 \\
\hline 1824 & 0.01310 & 1815 & 0.01316 & 1017 & 0.00805 \\
\hline 1003 & 0.01311 & 1920 & 0.01317 & 2052 & 0.00808 \\
\hline 2017 & 0.01337 & 2007 & 0.01343 & 2120 & 0.00622 \\
\hline 2008 & 0.01367 & 2058 & 0.01373 & 2173 & 0.00840 \\
\hline 2130 & 0.01400 & 2120 & 0.01406 & 2200 & 0.00861 \\
\hline 2189 & 0.01453 & 2179 & 0.01460 & 2301 & 0.00893 \\
\hline 2237 & 0.01510 & 2226 & 0.01517 & 2351 & 0.00928 \\
\hline 2312 & 0.01619 & 2301 & 0.01627 & 2431 & 0.00995 \\
\hline 2056 & 0.01746 & 2345 & 0.01755 & 2477 & 0.01074 \\
\hline 2080 & 0.01866 & 2069 & 0.01875 & 2502 & 0.01147 \\
\hline 2374 & 0.01857 & 2063 & 0.01966 & 2406 & 0.01203 \\
\hline 2403 & 0.02084 & 2391 & 0.002004 & 2526 & 0.01281 \\
\hline 2411 & 0.02165 & 2400 & 0.02176 & 2535 & 0.01331 \\
\hline 2430 & 0.00281 & 2418 & 0.00272 & 2554 & 0.01390 \\
\hline 2453 & 0.02380 & 2441 & 0.02301 & 2578 & 0.01463 \\
\hline 2525 & 0.00515 & 2513 & 0.02527 & 2654 & 0.01546 \\
\hline 2573 & 0.02642 & 2561 & 0.02655 & 2705 & 0.01624 \\
\hline 2633 & 0.02764 & 2020 & 0.0277 & 2768 & 0.01699 \\
\hline 2720 & 0.002872 & 2707 & 0.00888 & 2050 & 0.01768 \\
\hline 2834 & 0.02887 & 2820 & 0.05001 & 2970 & 0.01836 \\
\hline 2970 & 0.05087 & 2956 & 0.03102 & 3122 & 0.01898 \\
\hline 3131 & 0.00173 & 3116 & 0.03188 & 3292 & 0.01850 \\
\hline 3338 & 0.03268 & 3322 & 0.00284 & 3509 & 0.02000 \\
\hline 3538 & 0.03333 & 3521 & 0.03349 & 3719 & 0.02049 \\
\hline 3750 & 0.03350 & 3741 & 0.03366 & 3952 & 0.02000 \\
\hline 4082 & 0.03390 & 4013 & 0.03406 & 4238 & 0.02084 \\
\hline
\end{tabular}




\begin{tabular}{|c|c|c|c|c|c|}
\hline Po $(m)$ & $f(m)$ & Ro 0 & $f(0)$ & $\mathbf{P}_{\omega_{m}}$ & $t_{m}$ \\
\hline 4385 & 0.008400 & 4344 & 0.03477 & 4580 & 0.02127 \\
\hline 4703 & 0.08490 & 4740 & 0.03506 & 5006 & 0.02145 \\
\hline 52054 & 0.00585 & 5020 & 0.03582 & 5520 & 0.02182 \\
\hline 8713 & 0.05601 & 8085 & 0.05618 & 0005 & 0.00214 \\
\hline 6164 & $0.00 \operatorname{sen}$ & 8134 & 0.05082 & 6479 & 0.00241 \\
\hline 6742 & 0.05707 & 6710 & 0.03725 & 7087 & 0.002279 \\
\hline 7208 & 0.03723 & 2003 & 0.03741 & 7000 & 0.00280 \\
\hline$\pi 00$ & 0.03754 & 7732 & 0.03773 & 8167 & 0.02008 \\
\hline 6682 & 0.03790 & 6040 & 0.03808 & 9125 & 0.00350 \\
\hline 9415 & 0.08812 & 9370 & 0.03831 & 8007 & 0.02044 \\
\hline 10012 & 0.03835 & 8064 & 0.03854 & 10525 & 0.02358 \\
\hline 10730 & 0.03832 & 10578 & 0.03850 & 11279 & 0.02056 \\
\hline 11391 & 0.03878 & 11336 & 0.03896 & 11973 & 0.02384 \\
\hline 12332 & 0.03878 & 12273 & 0.03896 & 12963 & 0.02384 \\
\hline 13478 & 0.03875 & 13413 & 0.03893 & 14167 & 0.02382 \\
\hline 14468 & 0.03868 & 14398 & 0.03887 & 15208 & 0.02378 \\
\hline 15267 & 0.03881 & 15194 & 0.03800 & 10049 & 0.02386 \\
\hline 16445 & 0.05875 & 16566 & 0.03893 & 17287 & 0.00882 \\
\hline 17805 & 0.03855 & 17710 & 0.03873 & 18716 & 0.002870 \\
\hline 19375 & 0.03858 & 18283 & 0.03877 & 20567 & 0.003372 \\
\hline 20522 & 0.03852 & 20523 & 0.03870 & 21677 & 0.02368 \\
\hline 21986 & 0.05852 & 21881 & 0.03850 & 23111 & 0.02356 \\
\hline 24484 & 0.03803 & 24366 & 0.03821 & 25737 & 0.02388 \\
\hline 27242 & 0.03770 & 27112 & 0.03780 & 28636 & 0.020318 \\
\hline 20000 & 0.03748 & 20757. & 0.03766 & 31430 & 0.02304 \\
\hline 32151 & 0.08781 & 31807 & 0.03770 & 33797 & 0.02312 \\
\hline 35560 & 0.03767 & 35300 & 0.03785 & 37380 & 0.02316 \\
\hline 38731 & 0.05783 & 38545 & 0.03802 & $\$ 0713$ & 0.02028 \\
\hline 42948 & 0.03780 & 42742 & 0.03788 & 45145 & 0.02324 \\
\hline 40077 & 0.03800 & 46752 & 0.03828 & 49381 & 0.02342 \\
\hline 50004 & 0.03845 & -50660 & 0.03868 & 53508 & 0.02364 \\
\hline 54178 & 0.03888 & 53910 & 0.03006 & 50051 & 0.02350 \\
\hline 57812 & 0.03024 & 57534 & 0.03943 & 60770 & 0.02413 \\
\hline
\end{tabular}




\begin{tabular}{|c|c|c|c|c|c|}
\hline Po (m) & $f(m)$ & Ao $(1)$ & $f(0$ & $\mathbf{R}_{\mathrm{m}}$ & $t_{m}$ \\
\hline 00000 & 0.05054 & 02718 & 0.03973 & 6024 & 0.02431 \\
\hline erees1 & 0.01012 & 67555 & 0.04031 & 71354 & 0.02467 \\
\hline 75081 & 0.04057 & 75817 & 0.04078 & 70000 & 0.02494 \\
\hline 78680 & 0.04005 & 78311 & 0.04114 & 82715 & 0.00517 \\
\hline
\end{tabular}

Heat transfer $(A 20$, data set $(4)): \downarrow_{A}(m)=2.04, \downarrow_{A}(0)=2.05$

\begin{tabular}{|c|c|c|c|c|}
\hline Po $(m)$ & $N u(m)$ & Ro 0 & Nu 10 & $\mathbf{R o}_{m}$ \\
\hline 554 & 7.52 & 551 & 7.55 & 1129 \\
\hline 672 & 7.72 & 860 & 7.78 & 1167 \\
\hline 503 & 7.86 & 500 & 7.99 & 1209 \\
\hline 620 & 6.26 & 617 & 8.30 & 1265 \\
\hline 652 & 8.63 & 649 & 8.67 & 1330 \\
\hline 693 & 6.84 & 600 & 8.88 & 1414 \\
\hline 720 & 9.23 & 725 & 9.28 & 1487 \\
\hline 750 & 9.45 & 755 & 8.50 & 1540 \\
\hline . 782 & 9.70 & 788 & 9.75 & 1616 \\
\hline 835 & 10.00 & 831 & 10.05 & 1703 \\
\hline 802 & 10.40 & 888 & 10.45 & 1820 \\
\hline 241 & 10.69 & 036 & 10.74 & 1820 \\
\hline 896 & 10.82 & $\infty 91$ & 10.97 & 2032 \\
\hline 1065 & 11.14 & 1059 & 11.19 & 2172 \\
\hline 1127 & 11.33 & 1121 & 11.38 & 2000 \\
\hline 1185 & 11.50 & 1179 & $\$ 1.55$ & 2418 \\
\hline 1275 & 11.67 & 1250 & 11.73 & 2601 \\
\hline 1302 & 11.82 & 1355 & 11.88 & 2778 \\
\hline 1412 & 12.00 & 1435 & 1206 & 2043 \\
\hline 1563 & 12.30 & 1555 & 12.36 & 3188 \\
\hline 1673 & 12.62 & 1665 & 12.68 & 3413 \\
\hline 1780 & 13.11 & 1781 & 13.17 & 3651 \\
\hline 1874 & 13.63 & 1885 & 13.60 & 3023 \\
\hline 1843 & 13.83 & 1933 & 14.00 & 3984 \\
\hline 2019 & 14.30 & 2000 & 14.46 & 4119 \\
\hline
\end{tabular}




\begin{tabular}{|c|c|c|c|c|}
\hline$R \rightarrow(m)$ & Nu (m) & Po (1) & Nu (1) & $\boldsymbol{R}_{\mathrm{m}}$ \\
\hline 2100 & 14.87 & 2003 & 14.84 & 1002 \\
\hline 2147 & 15.21 & 2137 & 15.28 & 4381 \\
\hline 2020 & 15.78 & 2212 & 15.86 & 4536 \\
\hline 2311 & 16.59 & 2000 & 16.67 & 4716 \\
\hline 2410 & 17.27 & 2000 & 17.36 & 4918 \\
\hline 2400 & 18.07 & 2487 & 18.16 & 5000 \\
\hline 2006 & 18.91 & 2503 & 10.00 & 5317 \\
\hline 2776 & 20.13 & 2762 & 2023 & 5683 \\
\hline 2011 & 21.15 & 2007 & 2125 & 5020 \\
\hline 3050 & 22.13 & 3006 & 22.24 & 6223 \\
\hline$\$ 184$ & 23.31 & 3179 & 23.42 & 6517 \\
\hline 3383 & 24.82 & 3867 & 24.84 & 6003 \\
\hline 3501 & 26.05 & 3574 & 26.17 & 7326 \\
\hline 3803 & 27.40 & 3785 & 27.62 & 7760 \\
\hline se41 & 28.70 & 3002 & 28.84 & 8040 \\
\hline 4106 & 30.01 & 4006 & 30.15 & 637 \\
\hline 4300 & 31,60 & 4279 & 31.75 & 8773 \\
\hline 4532 & 33.20 & 4510 & 33.44 & 8246 \\
\hline 4082 & 34.06 & 4640 & 34.23 & 8512 \\
\hline 4854 & 35.37 & 4831 & 35.54 & 9004 \\
\hline 5004 & 37.01 & 5070 & 37.18 & 10394 \\
\hline 5357 & 38.86 & 5352 & 30.04 & 10030 \\
\hline 5062 & 10.88 & 5635 & 41.17 & 11552 \\
\hline 5054 & 43.08 & 5926 & 43.27 & 12149 \\
\hline 6227 & 44.83 & 6197 & 45.05 & 12704 \\
\hline 6500 & 47.04 & 6550 & 47.27 & 13446 \\
\hline 6883 & 49.05 & 6853 & 4920 & 14050 \\
\hline 7268 & 5120 & 7253 & B1.54 & 14820 \\
\hline 7000 & 54.00 & 7620 & \$4.35 & 15620 \\
\hline 8052 & 56.35 & 7894 & 56.62 & 16388 \\
\hline 8388 & 58.34 & 8347 & 58.62 & 17113 \\
\hline 8003 & 60.87 & 8860 & 61.17 & 18164 \\
\hline 8422 & 63.83 & 8377 & 64.14 & 19224 \\
\hline
\end{tabular}




\begin{tabular}{|c|c|c|c|c|}
\hline Po (m) & $N u(m)$ & Re 0 & Nu $(1)$ & $\mathbf{R o}_{m}$ \\
\hline 2000 & 67.12 & 5083 & 67.45 & 200200 \\
\hline 10421 & 70.43 & 10371 & 70.7 & 21202 \\
\hline 11251 & 74.80 & 11187 & 75.16 & 22054 \\
\hline 12043 & 78.21 & 11886 & 78.50 & 24572 \\
\hline 12846 & 82.54 & 12785 & 22.84 & 28210 \\
\hline 13884 & 87.84 & 13318 & 88.27 & 27018 \\
\hline 14555 & 2.11 & 14485 & 82.56 & zeoss \\
\hline 15508 & 97.34 & 15402 & 97.81 & 31688 \\
\hline 16478 & 101.21 & 16500 & 101.70 & 30020 \\
\hline 17855 & 108.02 & 17860 & 108.54 & 56633 \\
\hline 19248 & 113.50 & 19155 & 114.14 & 30270 \\
\hline 20256 & 118.27 & 20150 & 118.84 & 41328 \\
\hline 21978 & 126.05 & 21872 & 126.66 & 44841 \\
\hline 22950 & 130.86 & 22840 & 131.59 & 46824 \\
\hline 24101 & 137.03 & 23985 & 137.69 & 49172 \\
\hline 26075 & 145.02 & 25950 & 145.72 & 53200 \\
\hline 28191 & 151.42 & 28056 & 152.15 & 57518 \\
\hline 29002 & 150.22 & 29778 & 159.89 & 61049 \\
\hline 31782 & 167.79 & 31620 & 168.60 & 64843 \\
\hline 34021 & 176.56 & 33858 & 177.42 & $6: 9413$ \\
\hline 36887 & 186.19 & 36710 & 187.00 & 75259 \\
\hline 39458 & 185.24 & 30200 & 186.18 & 80505 \\
\hline 42149 & 204.00 & 41946 & 204.98 & 85095 \\
\hline 44326 & 212.25 & 44113 & 213.27 & 80437 \\
\hline 48230 & 225.26 & 47890 & 226.34 & 88402 \\
\hline 51802 & 238.30 & S1673 & 239.53 & 105935 \\
\hline 54449 & 249.08 & 54188 & 250.28 & 111002 \\
\hline 57710 & 202.85 & 57434 & 264.12 & 117745 \\
\hline 61253 & 274.46 & 60000 & 275.78 & 124974 \\
\hline 65014 & 286.37 & e4702 & 297.75 & 132647 \\
\hline 68034 & 295.64 & 67707 & 297.06 & 138807 \\
\hline 71345 & 304.99 & 71003 & 306.46 & 145563 \\
\hline 72879 & 308.69 & 72530 & 310.17 & 148694 \\
\hline
\end{tabular}


(c) transverse ring inserts with no clearance for flow near the wall

Friction (B2O, data set (5)): semicircular (radius $=2 \mathrm{~mm}), p / \theta=20, \theta / D=0.04, d_{m}=49.82 \mathrm{~mm}$ $\downarrow_{A}(m)=1.053, \downarrow_{1}(m)=0.615, \downarrow_{A}(0)=1.056, \downarrow_{1}(0)=0.612$

\begin{tabular}{|c|c|c|c|c|c|}
\hline Ao $(m)$ & $f(m)$ & no 0 & 10 & $\mathbf{P o}_{\mathrm{m}}$ & $t_{m}$ \\
\hline 834 & 0.00056 & 850 & 0.00568 & 877 & 0.01571 \\
\hline 802 & 0.02481 & 867 & 0.02493 & $\infty 08$ & 0.01525 \\
\hline 605 & 0.00200 & 600 & 0.02410 & 200 & 0.01474 \\
\hline 827 & 0.02316 & $\cos$ & 0.02520 & 975 & 0.01425 \\
\hline 257 & 0.00233 & $\cos 2$ & 0.00243 & 1006 & 0.01373 \\
\hline 892 & 0.02153 & 807 & 0.02163 & 1043 & 0.01323 \\
\hline 1034 & 0.02072 & 1000 & 0.02082 & 1087 & 0.01274 \\
\hline 1062 & 0.02018 & 1057 & 0.02027 & 1116 & 0.01241 \\
\hline 1089 & 0.01974 & 1003 & 0.01983 & 1144 & 0.01213 \\
\hline 1121 & 0.01887 & 1115 & 0.01896 & 1178 & 0.01160 \\
\hline 1158 & 0.01845 & 1152 & 0.01854 & 1217 & 0.01135 \\
\hline 1198 & 0.01788 & 1192 & 0.01797 & 1250 & 0.01090 \\
\hline 1230 & 0.01724 & 1224 & 0.01732 & 1293 & 0.01060 \\
\hline 1270 & 0.01678 & 1264 & 0.01687 & 1335 & 0.01032 \\
\hline 1321 & 0.01617 & 1314 & 0.01625 & 1388 & 0.00994 \\
\hline 1368 & 0.01555 & 1361 & 0.01563 & 1438 & 0.00056 \\
\hline 1428 & 0.01492 & 1422 & 0.01499 & 1500 & 0.00917 \\
\hline 1486 & 0.01441 & 1479 & 0.01448 & 1502 & 0.00886 \\
\hline 1540 & 0.01396 & 1533 & 0.01403 & 1619 & 0.00858 \\
\hline 1606 & 0.01346 & 1508 & 0.01352 & 1688 & 0.00827 \\
\hline 1689 & 0.01317 & 1681 & 0.01323 & 1775 & 0.00809 \\
\hline 1778 & 0.01302 & 1760 & 0.01508 & 1868 & 0.00800 \\
\hline 1855 & 0.01303 & 1846 & 0.01300 & 1850 & 0.00801 \\
\hline 1948 & 0.01320 & 1858 & 0.01326 & 2047 & 0.00811 \\
\hline 2004 & 0.01345 & 2005 & 0.01351 & 2170 & 0.00827 \\
\hline 2154 & 0.01385 & 2144 & 0.01302 & 2264 & 0.00852 \\
\hline 2180 & 0.01451 & 2170 & 0.01458 & 2002 & 0.00892 \\
\hline 2279 & 0.01540 & 2268 & 0.01547 & 2398 & 0.00947 \\
\hline 2334 & 0.01663 & 2323 & 0.01671 & 2454 & 0.01023 \\
\hline
\end{tabular}




\begin{tabular}{|c|c|c|c|c|c|}
\hline Ro $(m)$ & $f(m)$ & $P 0$ & 10 & $\mathbf{R}_{m}$ & $f_{m}$ \\
\hline 2001 & 0.01814 & 2370 & 0.01823 & 2513 & 0.01115 \\
\hline 2424 & 0.01989 & 2412 & 0.01948 & 2018 & 0.01182 \\
\hline 2467 & 0.02001 & 2456 & 0.02101 & 2504 & 0.01286 \\
\hline 2055 & 0.00231 & 2043 & 0.00272 & 2686 & 0.01300 \\
\hline 2865 & 0.00004 & 2843 & 0.02406 & 2791 & 0.01472 \\
\hline 2797 & 0.02004 & 278 & 0.00516 & 2041 & 0.01540 \\
\hline 2070 & 0.02818 & 2056 & 0.02650 & 3122 & 0.01600 \\
\hline 3153 & 0.00081 & 3138 & 0.02094 & 3014 & 0.01848 \\
\hline 3410 & 0.02774 & 8403 & 0.02787 & 3504 & 0.01705 \\
\hline 3006 & 0.02833 & 3878 & 0.02847 & 3885 & 0.01742 \\
\hline 4115 & 0.02889 & 4056 & 0.002003 & 4326 & 0.01776 \\
\hline 4390 & 0.02026 & 4378 & 0.02040 & 4624 & 0.01799 \\
\hline 4783 & 0.00064 & 4760 & 0.02078 & 5028 & 0.01822 \\
\hline 5161 & 0.02098 & 5136 & 0.03006 & 5425 & 0.01839 \\
\hline 6573 & 0.05002 & 5546 & 0.05037 & 5858 & 0.01858 \\
\hline 6148 & 0.05046 & 6118 & 0.05060 & 6463 & 0.01872 \\
\hline 6028 & 0.05046 & 6596 & 0.00000 & 6067 & 0.01872 \\
\hline 7281 & 0.05046 & 7246 & 0.03060 & 7653 & 0.01872 \\
\hline$\pi 82$ & 0.05046 & 7745 & 0.03060 & 8181 & 0.01872 \\
\hline 8097 & 0.05043 & 8657 & 0.03058 & 8827 & 0.01871 \\
\hline 8375 & 0.05043 & 9330 & 0.03058 & 8855 & 0.01871 \\
\hline 10255 & 0.05043 & 10206 & 0.05058 & 10779 & 0.01871 \\
\hline 11159 & 0.03059 & 11106 & 0.03084 & 11730 & 0.01887 \\
\hline 12144 & 0.05043 & 12086 & 0.03058 & 12765 & 0.01871 \\
\hline 13710 & 0.05015 & 13344 & 0.05020 & 14411 & 0.01853 \\
\hline 14592 & 0.08015 & 14522 & 0.03020 & 15338 & 0.01853 \\
\hline 15744 & 0.03002 & 15600 & 0.03016 & 16550 & 0.01846 \\
\hline 16786 & 0.000078 & 16705 & 0.02991 & 17845 & 0.01830 \\
\hline 182220 & 0.00000 & 18132 & 0.05014 & 19152 & 0.01844 \\
\hline 20160 & 0.00082 & 20072 & 0.02096 & 21201 & 0.01833 \\
\hline 21743 & 0.02861 & 21630 & 0.00976 & 22856 & 0.01821 \\
\hline 24028 & 0.02839 & 23913 & 0.00058 & 25258 & 0.01807 \\
\hline 26804 & 0.02946 & 26675 & 0.00050 & 28175 & 0.01811 \\
\hline
\end{tabular}




\begin{tabular}{|c|c|c|c|c|c|}
\hline$R(m)$ & $f(m)$ & Po 0 & 10 & $\boldsymbol{P o}_{\mathrm{m}}$ & $t_{m}$ \\
\hline 29773 & 0.00056 & 20300 & 0.02070 & 31206 & 0.01818 \\
\hline 33411 & 0.00081 & 85251 & 0.02976 & 98121 & 0.01821 \\
\hline$\$ 7500$ & 0.02040 & 37124 & 0.00093 & seet1 & 0.01813 \\
\hline 40524 & 0.00508 & 40350 & 0.02001 & 42508 & 0.01824 \\
\hline 43700 & 0.00006 & 45680 & 0.02831 & 46041 & 0.01824 \\
\hline 48609 & 0.008971 & 48450 & 0.02086 & 51184 & 0.01827 \\
\hline 50808 & 0.00000 & 52844 & 0.05004 & 55605 & 0.01838 \\
\hline 50253 & 0.05053 & 50003 & 0.05047 & 50131 & 0.01864 \\
\hline sorro & 0.05008 & 50483 & 0.05047 & 62028 & 0.01864 \\
\hline 65043 & 0.05008 & 64731 & 0.05042 & $\$ 8371$ & 0.01861 \\
\hline 60642 & 0.03050 & 60308 & 0.05073 & 73206 & 0.01880 \\
\hline 72681 & 0.03085 & 72332 & 0.03100 & 76309 & 0.01805 \\
\hline 74630 & 0.03103 & 74273 & 0.03118 & 78449 & 0.01008 \\
\hline
\end{tabular}

Heat transfer $(B 20$, data set $(5)): \downarrow_{A}(m)=1.899, \downarrow_{R}(D)=1.908$

\begin{tabular}{|c|c|c|c|c|}
\hline$R_{0}(m)$ & $\mathrm{Nu}(\mathrm{m})$ & Re (i) & Nu (0) & $\mathbf{R}_{\boldsymbol{e}_{m}}$ \\
\hline 1886 & 12.43 & 1877 & 12.49 & 3581 \\
\hline 1994 & 12.99 & 1985 & 13.05 & 3788 \\
\hline 2114 & 13.75 & 2104 & 13.82 & 4014 \\
\hline 2215 & 14.46 & 2205 & 14.53 & 4207 \\
\hline 2338 & 15.15 & 2327 & 15.23 & 4440 \\
\hline 2441 & 16.88 & 2450 & 16.06 & 4636 \\
\hline 2587 & 16.98 & 2575 & 07.06 & 4914 \\
\hline 2710 & 18.14 & 2697 & 18.23 & 5146 \\
\hline 2872 & 18.25 & 2858 & 19.34 & 5454 \\
\hline 3008 & 20.60 & 3068 & 20.70 & 5855 \\
\hline 3219 & 21.61 & 3204 & 21.71 & 6114 \\
\hline 3424 & 2200 & 3408 & 23.11 & 6503 \\
\hline 3604 & 24.18 & 3586 & 24.30 & 6844 \\
\hline 3784 & 25.48 & 3766 & 25.60 & 7187 \\
\hline 4000 & 28.70 & 3980 & 26.83 & 7596 \\
\hline 4165 & 28.14 & 4145 & 28.27 & 7800 \\
\hline
\end{tabular}




\begin{tabular}{|c|c|c|c|c|}
\hline Po (m) & $N u(m)$ & Ro (1) & Nu 10 & $\mathbf{P}_{m}$ \\
\hline 4824 & 20.34 & 4504 & 20.48 & 6212 \\
\hline 4480 & 20.54 & 4450 & 50.60 & 8500 \\
\hline 4702 & 3221 & 4750 & 22.37 & 8044 \\
\hline 8010 & 83.75 & 4005 & 83.01 & 9531 \\
\hline 824 & 35.27 & 5210 & 35.44 & $\infty 000$ \\
\hline 5457 & 86.54 & 5431 & 38.71 & 10563 \\
\hline 5050 & 30.02 & $\operatorname{ses} 2$ & 5921 & 11100 \\
\hline 0002 & 40.34 & 5094 & 40.54 & 11437 \\
\hline 0002 & 42.48 & 6382 & 42.60 & 12130 \\
\hline 6737 & 4.86 & 6704 & 45.08 & 12794 \\
\hline 7150 & 46.84 & 7116 & 47.17 & 13579 \\
\hline 7504 & 49.00 & 7468 & 49.32 & 14250 \\
\hline 8021 & 51.76 & 7983 & 52.01 & 15233 \\
\hline 8605 & 54.50 & 8563 & 54.85 & 16341 \\
\hline 8947 & 56.91 & 8904 & 57.18 & 16991 \\
\hline 2037 & 58.80 & 9193 & 50.08 & 17542 \\
\hline 9707 & 81.44 & 8081 & 61.73 & 18435 \\
\hline 10200 & 64.51 & 10160 & 64.82 & 19387 \\
\hline 10820 & 67.89 & 10768 & 68.21 & 20548 \\
\hline 11517 & 71.03 & 11462 & 71.38 & 21872 \\
\hline 12452 & 75.98 & 12392 & 76.34 & 20847 \\
\hline 13301 & 79.61 & 132037 & 79.99 & 25260 \\
\hline 14057 & 83.77 & 13990 & 84.18 & 26586 \\
\hline 14878 & 87.16 & 14807 & 87.58 & 20255 \\
\hline 15760 & 91.72 & 15094 & 92.16 & 20947 \\
\hline 16761 & 86:18 & 16681 & 86.64 & 31831 \\
\hline 17200 & 10222 & 17844 & 10271 & 34050 \\
\hline 18057 & 106.65 & 18068 & 107.17 & 38182 \\
\hline 20156 & 110.80 & 20050 & 111.42 & 38278 \\
\hline 21515 & 117.02 & 21412 & 147.58 & 40850 \\
\hline 22546 & 122.18 & 22458 & 122.77 & 42817 \\
\hline 23863 & 127.57 & 23748 & 128.10 & 45317 \\
\hline 25220 & 132.45 & 25099 & 133.09 & 47805 \\
\hline
\end{tabular}




\begin{tabular}{|c|c|c|c|c|}
\hline$R_{0}(m)$ & $\mathrm{Nu}(\mathrm{m})$ & $R(1)$ & Nu (1) & $\boldsymbol{P}_{\mathrm{m}}$ \\
\hline 26523 & 137.71 & 20806 & 138.38 & 50060 \\
\hline 20052 & 142.88 & 27917 & 143.67 & 58272 \\
\hline 20785 & 149.30 & 20652 & 150.11 & 58583 \\
\hline 31378 & 158.21 & 31228 & 153.86 & 50000 \\
\hline 33447 & 164.83 & 35286 & 165.17 & 60518 \\
\hline 35528 & 172.73 & 35355 & 173.56 & 67468 \\
\hline 38245 & 180.38 & 38000 & 18122 & 72031 \\
\hline 40503 & 187.78 & 40500 & 188.60 & 77001 \\
\hline 12812 & 194.83 & 40006 & 185.76 & 81300 \\
\hline 45803 & 203.57 & 45673 & 204.55 & 87156 \\
\hline 48367 & 213.76 & 48135 & 214.70 & 91853 \\
\hline 51263 & 222.56 & 61018 & 203.64 & 97364 \\
\hline 58550 & 231.40 & 53274 & 232.52 & 101650 \\
\hline 57547 & 242.82 & 57271 & 243.89 & 109287 \\
\hline 60262 & 254.61 & 50973 & 255.84 & 114444 \\
\hline 62072 & 202.85 & 02670 & 284.12 & 118589 \\
\hline 60933 & 274.65 & 68612 & 275.97 & 127111 \\
\hline 71140 & 235.86 & 70802 & 287.34 & 135107 \\
\hline 74500 & 297.11 & 74143 & 208.54 & 141482 \\
\hline 76916 & 305.21 & 76547 & $\mathbf{5 0 5 . 6 8}$ & 146071 \\
\hline 70693 & 313.09 & 72311 & 314.60 & 151345 \\
\hline
\end{tabular}

Friction (B80, data set $(7)$ ): semicircular (radius $=2 \mathrm{~mm}$ ), $\mathrm{p} / \mathrm{\theta}=80, \theta / \mathrm{D}=0.04, \mathrm{~d}_{\mathrm{m}}=49.95 \mathrm{~mm}$ $\downarrow_{R}(m)=0.826, \downarrow_{1}(m)=0.842, \downarrow_{R}(i)=0.828, \downarrow_{1}(i)=0.839$

\begin{tabular}{|c|c|c|c|c|c|}
\hline$R 0(m)$ & $f(m)$ & $R(i)$ & $f(0)$ & $R_{m}$ & $f_{m}$ \\
\hline 600 & 0.04215 & 400 & 0.04224 & 413 & 0.03548 \\
\hline 520 & 0.04057 & 519 & 0.04066 & 420 & 0.03415 \\
\hline 546 & 0.03878 & 544 & 0.03886 & 451 & 0.03264 \\
\hline 562 & 0.03777 & 560 & 0.03785 & 464 & 0.03179 \\
\hline 677 & 0.05682 & 576 & 0.03690 & 477 & 0.03099 \\
\hline 505 & 0.03589 & 594 & 0.03597 & 492 & 0.03021 \\
\hline 621 & 0.03445 & 620 & 0.03453 & 513 & 0.02900 \\
\hline 638 & 0.03333 & 637 & 0.03340 & 527 & 0.02805 \\
\hline
\end{tabular}




\begin{tabular}{|c|c|c|c|c|c|}
\hline Ro $(m)$ & $f(m)$ & Po 0 & 10 & $\boldsymbol{R}_{\boldsymbol{\rho}_{m}}$ & in \\
\hline 607 & 0.00286 & $\infty 85$ & 0.05273 & 842 & 0.02740 \\
\hline 668 & 0.00200 & 657 & 0.03207 & 852 & 0.02893 \\
\hline$\infty 82$ & 0.03127 & 680 & 0.03134 & 583 & 0.02032 \\
\hline 605 & 0.05003 & 604 & 0.00073 & 874 & 0.00581 \\
\hline 715 & 0.00000 & 714 & 0.00896 & 601 & 0.02516 \\
\hline 738 & 0.00607 & 796 & 0.002003 & $\infty$ & 0.02438 \\
\hline 788 & 0.02702 & 705 & 0.02768 & 603 & 0.00325 \\
\hline 704 & 0.02678 & 700 & 0.02684 & $\mathbf{\infty 6 8}$ & 0.02255 \\
\hline 820 & $0.00 \times 53$ & 818 & 0.00589 & 677 & 0.02183 \\
\hline 844 & 0.02517 & 842 & 0.02523 & 697 & 0.02119 \\
\hline 883 & 0.02456 & 881 & 0.02461 & 730 & 0.02067 \\
\hline 915 & 0.000348 & 913 & 0.02353 & 756 & 0.01976 \\
\hline 944 & 0.02275 & 842 & 0.00280 & 780 & 0.01915 \\
\hline 975 & 0.00216 & 873 & 0.02221 & 205 & 0.01865 \\
\hline 1003 & 0.02096 & 1021 & 0.02101 & 845 & 0.01765 \\
\hline 1000 & 0.01800 & 1078 & 0.01973 & 602 & 0.01657 \\
\hline 1124 & 0.01806 & 1121 & 0.01900 & 828 & 0.01596 \\
\hline 1163 & 0.01841 & 1160 & 0.01845 & 860 & 0.01549 \\
\hline 1225 & 0.01750 & 1222 & 0.01743 & 1012 & 0.01464 \\
\hline 1264 & 0.01600 & 1261 & 0.01697 & 1044 & 0.01425 \\
\hline 1307 & 0.01640 & 1304 & 0.01643 & 1080 & 0.01380 \\
\hline 1320 & 0.01611 & 1320 & 0.01614 & 1083 & 0.01356 \\
\hline 1357 & 0.01583 & 1354 & 0.01587 & 1121 & 0.01333 \\
\hline 1307 & 0.01533 & 1394 & 0.01556 & 1154 & 0.01290 \\
\hline 1433 & 0.01499 & 1430 & 0.01503 & 1184 & 0.01262 \\
\hline 1500 & 0.01448 & 1505 & 0.01451 & 1246 & 0.01218 \\
\hline 1560 & 0.01376 & 1568 & 0.01379 & 1296 & 0.01158 \\
\hline 1647 & 0.01383 & 1644 & 0.01336 & 1361 & 0.01122 \\
\hline 1728 & 0.01286 & 1724 & 0.01288 & 1427 & 0.01082 \\
\hline 1790 & 0.01201 & 1785 & 0.01234 & 1486 & 0.01038 \\
\hline 1908 & 0.01183 & 1804 & 0.01185 & 1578 & 0.01004 \\
\hline 1973 & 0.01151 & 1968 & 0.01153 & 1630 & 0.00069 \\
\hline 2055 & 0.01111 & 2051 & 0.01113 & 1698 & 0.00035 \\
\hline
\end{tabular}




\begin{tabular}{|c|c|c|c|c|c|}
\hline$R_{0}(m)$ & $f(m)$ & Po 0 & 10 & $\boldsymbol{A n}_{\mathrm{m}}$ & $i_{m}$ \\
\hline 2152 & 0.01005 & 2148 & 0.01088 & 1778 & 0.00014 \\
\hline 2025 & 0.01000 & 2028 & 0.01082 & 1844 & 0.00002 \\
\hline 20340 & 0.01000 & 22035 & 0.01042 & 1833 & 0.00875 \\
\hline 2449 & 0.01000 & 2443 & 0.01052 & 2000 & 0.00867 \\
\hline 2510 & 0.01088 & 2055 & 0.01041 & 2000 & 0.00874 \\
\hline 2003 & 0.01000 & 2000 & 0.01071 & 2150 & 0.00000 \\
\hline 2009 & 0.01123 & 2093 & 0.01125 & 2020 & 0.00045 \\
\hline 2760 & 0.01180 & 2753 & 0.01191 & 2270 & 0.01001 \\
\hline 2051 & 0.01245 & 2844 & 0.01247 & 2055 & 0.01048 \\
\hline 2887 & 0.01284 & 2881 & 0.01297 & 2385 & 0.01000 \\
\hline 2937 & 0.01371 & 22031 & 0.01374 & 2426 & 0.01154 \\
\hline 5047 & 0.01420 & 3040 & 0.01423 & 2017 & 0.01185 \\
\hline 3139 & 0.01453 & 3132 & 0.01456 & 2593 & 0.01223 \\
\hline 3268 & 0.01499 & 3261 & 0.01503 & 2609 & 0.01282 \\
\hline 3437 & 0.01503 & 3429 & 0.01507 & 2839 & 0.01265 \\
\hline 3683 & 0.01518 & 3675 & 0.01522 & 3042 & 0.01278 \\
\hline 3893 & 0.01523 & 3885 & 0.01528 & 3216 & 0.01282 \\
\hline 4151 & 0.01525 & 4142 & 0.01529 & 3429 & 0.01284 \\
\hline 4544 & 0.01527 & 4534 & 0.01530 & 3754 & 0.01285 \\
\hline 4899 & 0.01520 & 4888 & 0.01532 & 4047 & 0.01287 \\
\hline 5254 & 0.01527 & 5243 & 0.01530 & 4340 & 0.01285 \\
\hline 5684 & 0.01527 & 5671 & 0.01530 & 4695 & 0.01285 \\
\hline 6117 & 0.01524 & 6103 & 0.01527 & 5053 & $0.012 a 3$ \\
\hline 0638 & 0.01511 & 6524 & 0.01814 & 5401 & 0.01272 \\
\hline 7060 & 0.01501 & 7045 & 0.01504 & 5832 & 0.01263 \\
\hline 7743 & 0.01505 & $\pi 26$ & 0.01508 & 6396 & 0.01288 \\
\hline 8000 & 0.01498 & 8372 & 0.01490 & 6950 & 0.01259 \\
\hline $830 s$ & 0.01484 & 8283 & 0.01488 & 7685 & 0.01249 \\
\hline 10168 & 0.01475 & 10145 & 0.01470 & 8000 & 0.01242 \\
\hline 10970 & 0.01450 & 10046 & 0.01462 & 8082 & 0.01228 \\
\hline 11838 & 0.01453 & 11912 & 0.01456 & 8861 & 0.01223 \\
\hline 12653 & 0.01442 & 12635 & 0.01445 & 10460 & 0.01214 \\
\hline 13432 & 0.01436 & 13402 & 0.01430 & 11005 & 0.01200 \\
\hline
\end{tabular}




\begin{tabular}{|c|c|c|c|c|c|}
\hline Po $(m)$ & $f(m)$ & $R 0$ & 10 & $\boldsymbol{n} \boldsymbol{\bullet}_{m}$ & $t_{m}$ \\
\hline 14431 & 0.01425 & 14500 & 0.01428 & 11820 & 0.01189 \\
\hline 18560 & 0.01408 & 15020 & 0.01400 & 12687 & 0.01183 \\
\hline 16886 & 0.01384 & 16849 & 0.01387 & 13783 & 0.01165 \\
\hline 18173 & 0.01373 & 18133 & 0.01376 & 15012 & 0.01155 \\
\hline 18743 & 0.01380 & 19700 & 0.01363 & 16508 & 0.01145 \\
\hline 21818 & 0.01351 & 21770 & 0.01354 & 18002 & 0.01137 \\
\hline 24380 & 0.01352 & 24523 & 0.01335 & 20138 & 0.01121 \\
\hline 20007 & 0.01312 & 20007 & 0.01315 & 20202 & 0.01104 \\
\hline 20746 & 0.01300 & 20082 & 0.01312 & 24572 & 0.01102 \\
\hline 32125 & 0.01304 & 32055 & 0.01307 & 28556 & 0.01008 \\
\hline 35186 & 0.01302 & 35119 & 0.01305 & 29073 & 0.01006 \\
\hline 37976 & 0.01293 & 37892 & 0.01296 & 31360 & 0.01089 \\
\hline 41221 & 0.01287 & 41130 & 0.01289 & 34050 & 0.01083 \\
\hline 44632 & 0.01284 & 44534 & 0.01287 & 36867 & 0.01081 \\
\hline 48732 & 0.01288 & 48025 & 0.01291 & 40254 & 0.01084 \\
\hline 54176 & 0.01286 & 54057 & 0.01288 & 4451 & 0.01082 \\
\hline 58560 & 0.01275 & 58431 & 0.01277 & 48372 & 0.01073 \\
\hline 62806 & 0.01278 & 62668 & 0.01281 & 51879 & 0.01076 \\
\hline 68638 & 0.01275 & 68487 & 0.01277 & 56697 & 0.01073 \\
\hline 73116 & 0.01263 & 72055 & 0.01266 & 60306 & 0.01063 \\
\hline 78692 & 0.01262 & 78510 & 0.01264 & 65002 & 0.01062 \\
\hline
\end{tabular}

Heat transfer $(B 80$, data set $(7)): \downarrow_{R}(m)=1.639, \downarrow_{R}(1)=1.643$

\begin{tabular}{|r|r|r|r|r|}
\hline$R_{0}(\mathrm{~m})$ & $N_{u}(\mathrm{~m})$ & $R_{0}(0)$ & $N_{u}(0)$ & $R_{m}$ \\
\hline 4191 & 20.40 & 4182 & 20.45 & 6871 \\
\hline 4398 & 21.41 & 4380 & 21.45 & 7211 \\
\hline 4688 & 22.75 & 4678 & 22.80 & 7686 \\
\hline 4983 & 23.90 & 4972 & 24.05 & 8170 \\
\hline 5373 & 25.53 & 5361 & 25.50 & 8808 \\
\hline 5788 & 27.33 & 5775 & 27.30 & 9469 \\
\hline 6161 & 28.99 & 6147 & 29.05 & 10100 \\
\hline 6548 & 30.57 & 6534 & 30.63 & 10735 \\
\hline
\end{tabular}




\begin{tabular}{|c|c|c|c|c|}
\hline $\mathrm{Bo}_{0}(\mathrm{~m})$ & Nu $(m)$ & Po 0 & Nu $(1)$ & $\boldsymbol{R}_{m}$ \\
\hline 6040 & 91.04 & 0005 & 22.01 & 11378 \\
\hline 7001 & 33.54 & rans & $\mathbf{s 3 . 6 1}$ & 12008 \\
\hline 7688 & 35.37 & 7868 & 35.44 & 12028 \\
\hline 8528 & 36.03 & 0310 & 37.01 & 13654 \\
\hline 8752 & 38.45 & 8733 & $\mathbf{3 8 . 5 3}$ & 14340 \\
\hline 9126 & 40.00 & 9106 & 40.18 & 14062 \\
\hline 0352 & 42.18 & 9631 & 42.28 & 15824 \\
\hline $\operatorname{cose}$ & 43.06 & 8880 & 43.15 & 10234 \\
\hline $10<03$ & 4.48 & 10081 & 4.58 & 16891 \\
\hline 10782 & 46.19 & 10758 & 4620 & 17676 \\
\hline 11331 & 48.02 & 11306 & 48.13 & 18576 \\
\hline 11773 & 49.75 & 11747 & 49.86 & 18501 \\
\hline 12585 & 53.14 & 12557 & 53.25 & 20632 \\
\hline 13254 & 54.00 & 13225 & 55.02 & 21728 \\
\hline 13880 & 57.03 & 13858 & 57.16 & 22770 \\
\hline 14868 & 59.83 & 14835 & 60.06 & 24374 \\
\hline 15547 & $e .31$ & 15513 & 62.45 & 25488 \\
\hline 16572 & $\$ 5.76$ & 16536 & 65.90 & 27160 \\
\hline 17614 & $\infty .00$ & 17576 & 69.15 & $288 \pi$ \\
\hline 18643 & 72.25 & 18602 & 72.41 & 30564 \\
\hline 18584 & 74.80 & 18521 & 74.97 & 32074 \\
\hline 20546 & 77.61 & 20501 & 77.78 & 33683 \\
\hline 21500 & 80.20 & 21453 & 80.41 & 35247 \\
\hline 22197 & 82.50 & 22148 & 82.49 & 36390 \\
\hline 20099 & 84.85 & 22048 & 85.04 & 37704 \\
\hline 28812 & 87.16 & 23760 & 87.35 & 39038 \\
\hline 24812 & 89.88 & 24758 & 80.18 & 40677 \\
\hline 25526 & 824 & 25470 & 82.64 & 41848 \\
\hline 26448 & 84.00 & 26390 & 84.50 & 43350 \\
\hline 27853 & 83.31 & 27782 & 88.53. & 45663 \\
\hline 20002 & 101.71 & 20028 & 101.03 & 48002 \\
\hline 50730 & 105.60 & 50672 & 405.83 & 50395 \\
\hline 31804 & 100.25 & 31734 & 109.49 & 52140 \\
\hline
\end{tabular}




\begin{tabular}{|c|c|c|c|c|}
\hline Po $(m)$ & $N u(m)$ & R 0 & Nu 0 & $\boldsymbol{R e}_{\mathrm{m}}$ \\
\hline 50000 & 112.63 & $\sec 7$ & 112.88 & 54000 \\
\hline 34778 & 116.44 & 34701 & 116.60 & 57015 \\
\hline 37006 & 122.01 & 37015 & 122.28 & 60817 \\
\hline se654 & 125.43 & 80570 & 125.70 & 63571 \\
\hline soese & 128.30 & 30530 & 128.58 & 64064 \\
\hline 41057 & 131.43 & 40067 & 131.71 & 67500 \\
\hline 42000 & 134.91 & 42506 & 135.21 & 09987 \\
\hline 44673 & 138.80 & 44575 & 130.18 & 732038 \\
\hline 46715 & 142.98 & 46812 & 143.20 & 78585 \\
\hline 40232 & 147.71 & 40124 & 148.04 & 80712 \\
\hline 51400 & 152.49 & 51206 & 152.83 & 84281 \\
\hline 54759 & 160.47 & 54630 & 160.82 & 89773 \\
\hline 57302 & 165.31 & 5717 & 165.68 & 83043 \\
\hline 60777 & 171.88 & 60644 & 172.25 & 89640 \\
\hline 62793 & 176.56 & 62655 & 176.95 & 100245 \\
\hline 65523 & 180.48 & 65379 & 180.88 & 107420 \\
\hline 68469 & 186.18 & 68319 & 186.60 & 112250 \\
\hline 70490 & 191.68 & 70335 & 192.10 & 115502 \\
\hline 72673 & 105.38 & 72513 & 185.81 & 119141 \\
\hline 74608 & 200.00 & 74442 & 200.44 & 122310 \\
\hline 76318 & 202.56 & 76151 & 203.01 & 125118 \\
\hline 77905 & 204.87 & $\pi 733$ & 205.32 & 127718 \\
\hline
\end{tabular}


Friction (D2, data set (G)): semicircular (radius $=4 \mathrm{~mm}$ ), $p / \theta=2, e / D=0.08, d_{m}=43.85 \mathrm{~mm}$ $\downarrow_{A}(m)=0.737, \downarrow_{1}(m)=0.8, \downarrow_{A}(i)=0.841, \downarrow_{1}(0)=0.702$

\begin{tabular}{|c|c|c|c|c|c|}
\hline Po $(m)$ & $1(m)$ & $P 0$ & 10 & $\mathrm{Am}_{\mathrm{m}}$ & $t_{n}$ \\
\hline 1202 & 0.01786 & 1053 & 0.02016 & 886 & 0.01414 \\
\hline 1268 & 0.01711 & 1102 & 0.01933 & 827 & 0.01371 \\
\hline 1301 & 0.01641 & 1140 & 0.01873 & 850 & 0.01315 \\
\hline 1348 & 0.01585 & 1181 & 0.01800 & $\infty 28$ & 0.01270 \\
\hline 1376 & 0.01550 & 1205 & 0.01770 & 1014 & 0.01242 \\
\hline 1438 & 0.01478 & 1250 & 0.01687 & 1000 & 0.01184 \\
\hline 1510 & 0.01422 & 1323 & 0.01624 & 1112 & 0.01140 \\
\hline 1582 & 0.01380 & 1500 & 0.01575 & $\$ 151$ & 0.01105 \\
\hline 1029 & 0.01335 & 1427 & 0.01524 & 1200 & 0.01069 \\
\hline 1687 & 0.01300 & 1478 & 0.01484 & 1243 & 0.01041 \\
\hline 1765 & 0.01254 & 1546 & 0.01432 & 1301 & 0.01005 \\
\hline 1838 & 0.01210 & 1610 & 0.01382 & 1354 & 0.00070 \\
\hline 1816 & 0.01170 & 1679 & 0.01335 & 1412 & 0.00937 \\
\hline 1891 & 0.01131 & 1744 & 0.01201 & 1467 & 0.00906 \\
\hline 2087 & 0.01100 & 1828 & 0.01256 & 1538 & 0.00881 \\
\hline 2193 & 0.01065 & 1821 & 0.01216 & 1616 & 0.00853 \\
\hline 2262 & 0.01040 & 1881 & 0.01187 & 1666 & 0.00833 \\
\hline 2344 & 0.01020 & 2053 & 0.01174 & 1727 & 0.00824 \\
\hline 2436 & 0.01020 & 2134 & 0.01164 & 1785 & 0.00817 \\
\hline 2564 & 0.01016 & 2246 & 0.01150 & 1889 & 0.00814 \\
\hline 2671 & 0.01015 & 2040 & 0.01150 & 1868 & 0.00813 \\
\hline 2743 & 0.01014 & 2403 & 0.01158 & 2021 & 0.00812 \\
\hline 2817 & 0.01023 & 2467 & 0.01167 & 2075 & 0.00819 \\
\hline 2065 & 0.01036 & 2597 & 0.01182 & 2184 & 0.00850 \\
\hline 5013 & 0.01065 & 2630 & 0.01216 & 2200 & 0.00853 \\
\hline 3078 & 0.01100 & 2698 & 0.01256 & 2268 & 0.00881 \\
\hline 3147 & 0.01142 & 2757 & 0.01304 & 2319 & 0.00915 \\
\hline 3160 & 0.01182 & 2785 & 0.01361 & 2343 & 0.00955 \\
\hline 3260 & 0.01262 & 2855 & 0.01440 & 2401 & 0.01011 \\
\hline 3290 & 0.01350 & 2882 & 0.01518 & 2424 & 0.01066 \\
\hline 3370 & 0.01381 & 2952 & 0.01576 & 2483 & 0.01106 \\
\hline 3443 & 0.01438 & 5016 & 0.01642 & 2536 & 0.01152 \\
\hline
\end{tabular}




\begin{tabular}{|c|c|c|c|c|c|}
\hline$A 0(m)$ & $f(m)$ & Po 0 & 10 & $\mathbf{R o}_{\mathrm{m}}$ & $f_{m}$ \\
\hline 3404 & 0.01497 & 2000 & 0.01700 & 2667 & 0.01190 \\
\hline 3000 & 0.01557 & 3163 & 0.01780 & 2051 & 0.01266 \\
\hline 8080 & 0.01641 & 200 & 0.01873 & 2718 & 0.01315 \\
\hline 5857 & 0.01701 & $\operatorname{ses} 1$ & 0.01842 & 2827 & 0.01362 \\
\hline 8084 & 0.01701 & 3472 & 0.01898 & 2000 & 0.01402 \\
\hline 414 & 0.01784 & 3050 & 0.020068 & 3050 & 0.01420 \\
\hline 4308 & 0.01813 & 8772 & 0.00000 & 3172 & 0.01452 \\
\hline 4478 & 0.01856 & $\sec 20$ & 0.002008 & 3009 & 0.01471 \\
\hline 4758 & 0.01840 & 4160 & 0.02110 & 2491 & 0.01481 \\
\hline 4049 & 0.01842 & 4385 & 0.02103 & 3846 & 0.01476 \\
\hline 5178 & 0.01834 & 4556 & 0.020094 & 3815 & 0.01470 \\
\hline 5488 & 0.01828 & 4007 & 0.02087 & 4043 & 0.01465 \\
\hline 5876 & 0.01822 & 5147 & 0.02080 & 4320 & 0.01460 \\
\hline 6276 & 0.01813 & 5497 & 0.00069 & 4023 & 0.01452 \\
\hline 6754 & 0.01807 & 5916 & 0.02002 & 4976 & 0.01447 \\
\hline 7182 & 0.01816 & 6291 & 0.02073 & 5291 & 0.01455 \\
\hline 7547 & 0.01813 & 8311 & 0.02009 & 5550 & 0.01452 \\
\hline 8157 & 0.01805 & 7145 & 0.02061 & 0000 & 0.01446 \\
\hline 8907 & 0.01822 & 7002 & 0.002080 & 6582 & 0.01460 \\
\hline 8680 & 0.01817 & 8461 & 0.02075 & 7116 & 0.01456 \\
\hline 10396 & 0.01850 & 9106 & 0.00000 & 7650 & 0.01466 \\
\hline 11074 & 0.01833 & 9700 & 0.00002 & 8158 & 0.01468 \\
\hline 11809 & 0.01841 & 10502 & 0.02101 & 8833 & 0.01475 \\
\hline 12002 & 0.01856 & 11203 & 0.02119 & 9498 & 0.01487 \\
\hline 13698 & 0.01858 & 11800 & 0.02121 & 10092 & 0.01488 \\
\hline 14780 & 0.01858 & 12946 & 0.02121 & 10889 & 0.01488 \\
\hline 16139 & 0.01803 & 14137 & 0.02126 & 11800 & 0.01402 \\
\hline 17881 & 0.01871 & 15603 & 0.02136 & 13173 & 0.01490 \\
\hline 19120 & 0.01893 & 10758 & 0.02161 & 14092 & 0.01517 \\
\hline 20083 & 0.01886 & 17502 & 0.02165 & 14785 & 0.01518 \\
\hline 21031 & 0.01908 & 18422 & 0.02178 & 15404 & 0.01527 \\
\hline 22672 & 0.01808 & 19860 & 0.02176 & 16703 & 0.01527 \\
\hline 24991 & 0.01919 & 21801 & 0.02181 & 18411 & 0.01537 \\
\hline
\end{tabular}




\begin{tabular}{|c|c|c|c|c|c|}
\hline$R_{0}(m)$ & $f(m)$ & Po 0 & 10 & $\boldsymbol{A} \mathbf{a}_{\mathrm{m}}$ & $t_{m}$ \\
\hline 208405 & 0.01957 & 20200 & 0.02211 & 16512 & 0.01522 \\
\hline 27073 & 0.01022 & 24503 & 0.00240 & 20808 & 0.01572 \\
\hline 30011 & 0.01070 & 26551 & 0.00249 & 20301 & 0.01578 \\
\hline 33755 & 0.01884 & 20568 & 0.02265 & 24868 & 0.01580 \\
\hline 80205 & 0.00002 & 31740 & 0.00286 & 26095 & 0.01604 \\
\hline 98085 & 0.00007 & 205808 & 0.00082 & 28485 & 0.01808 \\
\hline 42655 & 0.002028 & 37534 & 0.00313 & 31424 & 0.01623 \\
\hline 45749 & 0.03047 & 40074 & 0.00337 & 35704 & 0.01840 \\
\hline 51185 & 0.02075 & 44818 & 0.00059 & 37694 & 0.01863 \\
\hline 55400 & 0.02104 & 48606 & 0.02402 & 40880 & 0.01885 \\
\hline 60334 & 0.02127 & 52349 & 0.02428 & 44448 & 0.01704 \\
\hline 64655 & 0.02162 & 56535 & 0.02468 & 47632 & 0.01732 \\
\hline 70350 & 0.02197 & 61631 & 0.02508 & 51835 & 0.01780 \\
\hline 76111 & 0.02238 & 66659 & 0.02555 & 56072 & 0.01783 \\
\hline 81076 & 0.02308 & 71019 & 0.02635 & 50730 & 0.01849 \\
\hline 84686 & 0.02342 & 74180 & 0.02673 & 62389 & 0.01876 \\
\hline 89215 & 0.02374 & 78147 & 0.02710 & 65725 & 0.01802 \\
\hline
\end{tabular}

Heat transfer $(D 2$, data set $(6)): \downarrow_{R}(m)=1.510, \downarrow_{A}(0)=1.724$

\begin{tabular}{|c|c|c|c|c|}
\hline$R_{0}(m)$ & $\mathrm{Nu}(\mathrm{m})$ & Pe (I) & Nu (1) & $\mathbf{P}_{m}$ \\
\hline 620 & 6.39 & 543 & 7.30 & 236 \\
\hline 642 & 6.66 & 562 & 7.60 & 970 \\
\hline 679 & 6.97 & 505 & 7.96 & 1025 \\
\hline 718 & 7.33 & 650 & 8.37 & 1086 \\
\hline 782 & 7.71 & 687 & 8.80 & 1150 \\
\hline 801 & 8.07 & 702 & 021 & 1210 \\
\hline 858 & 8.53 & 752 & 0.74 & 1296 \\
\hline 807 & 8.80 & 783 & 10.04 & 1355 \\
\hline 087 & 9.22 & 847 & 10.53 & 1461 \\
\hline 1005 & 8.53 & 898 & 10.88 & 1548 \\
\hline 1074 & 9.80 & 940 & 11.18 & 1622 \\
\hline 1148 & 10.17 & 1005 & 11.61 & 1733 \\
\hline
\end{tabular}




\begin{tabular}{|c|c|c|c|c|}
\hline Pos (m) & $N u(m)$ & Bu 0 & Nu 0 & $\mathbf{p}_{m}$ \\
\hline 1215 & 10.38 & 1084 & 11.84 & 1835 \\
\hline 1800 & 10.57 & 1146 & 12.06 & 1976 \\
\hline 1901 & 10.61 & 1218 & 12.12 & 2101 \\
\hline 1472 & 10.70 & 1200 & 12.32 & 2023 \\
\hline 1534 & 10.70 & 1344 & 1222 & 2317 \\
\hline 1818 & 10.84 & 1417 & 12.38 & 2443 \\
\hline 1747 & 11.03 & 1580 & 12.60 & 2838 \\
\hline 1872 & 11.13 & 1640 & 12.70 & 2028 \\
\hline 1938 & 1120 & 1737 & 12.84 & 2005 \\
\hline 2140 & 11.42 & 1882 & 13.04 & 3246 \\
\hline 2271 & 11.40 & 1800 & 13.12 & 3430 \\
\hline 2450 & 11.60 & 2154 & 13.24 & 3713 \\
\hline 2810 & 11.63 & 2286 & 13.27 & 3941 \\
\hline 2727 & 11.63 & 20389 & 13.27 & 4118 \\
\hline 2797 & 11.61 & 2450 & 13.26 & 4025 \\
\hline 2831 & 11.64 & 2400 & 1328 & 4276 \\
\hline 2874 & 12.01 & 2017 & 13.71 & 4340 \\
\hline 2050 & 12.79 & 2584 & 14.81 & 446 \\
\hline 3007 & 13.41 & 2634 & 15.31 & 4542 \\
\hline 3025 & 14.04 & 2702 & 16.03 & 4650 \\
\hline 3134 & 14.52 & 2745 & 16.58 & 4733 \\
\hline 3242 & 15.22 & 2840 & 17.37 & 4008 \\
\hline 3590 & 16.05 & 2070 & 18.32 & 5120 \\
\hline 3400 & 16.83 & 3057 & 18.21 & 5271 \\
\hline 3847 & 17.60 & 3185 & 20.32 & 5508 \\
\hline 9788 & 18.63 & 3027 & 2127 & 5736 \\
\hline 3088 & 19.65 & 3494 & 2243 & 0023 \\
\hline 4191 & 20.63 & 3871 & 23.58 & 8350 \\
\hline 4349 & 21.45 & 3800 & 24.40 & 6568 \\
\hline $455 t$ & 22.61 & 3908 & 25.81 & 6873 \\
\hline 4730 & 20.41 & 4151 & 28.72 & 7156 \\
\hline 4945 & 24.32 & 4501 & 27.78 & 7468 \\
\hline 5008 & 24.80 & 4485 & 28.32 & 7699 \\
\hline
\end{tabular}




\begin{tabular}{|c|c|c|c|c|}
\hline$P(m)$ & $\mathrm{Nu}(\mathrm{m})$ & Po 0 & Nu 0 & $\mathbf{R o}_{m}$ \\
\hline 5415 & 28.14 & 4743 & 20.84 & 8177 \\
\hline 5550 & 26.83 & 4800 & 30.81 & 8055 \\
\hline 5804 & 28.34 & 5084 & 32.35 & 8766 \\
\hline 0014 & 20.58 & 5268 & 33.51 & 0082 \\
\hline 6205 & 0.31 & S4a5 & 34.60 & $\$ 2570$ \\
\hline 6567 & 22.05 & 5752 & 38.50 & $\bullet 918$ \\
\hline 6709 & 3285 & 6047 & 37.61 & 100054 \\
\hline 7000 & 34.33 & 6210 & 89.10 & 10707 \\
\hline 7377 & 36.30 & cher & $\$ 0.40$ & 11141 \\
\hline 7720 & 38.80 & 6762 & 42.01 & 11658 \\
\hline 8021 & 38.18 & 7006 & 49.58 & 12114 \\
\hline 2405 & 40.17 & 7442 & 45.86 & 12830 \\
\hline 8840 & 41.80 & 7743 & 47.72 & 13350 \\
\hline 0276 & 43.52 & 8126 & 49.68 & 14010 \\
\hline 0632 & 45.44 & 8437 & 51.87 & 14546 \\
\hline 10101 & 47.41 & 8848 & 5.13 & 15255 \\
\hline 10577 & 49.61 & $\infty 265$ & 56.63 & 15974 \\
\hline 11060 & 51.88 & $\cos 5$ & 60.34 & 16716 \\
\hline 11525 & 53.50 & 10006 & 61.18 & 17406 \\
\hline 12155 & 56.03 & 10647 & 63.97 & 18357 \\
\hline 12692 & 58.51 & 11118 & 66.79 & 19160 \\
\hline 13169 & 60.53 & 11536 & 69.10 & 19889 \\
\hline 13481 & 01.70 & 11800 & 70.54 & 20360 \\
\hline 14208 & 65.20 & 12445 & 74.44 & 21457 \\
\hline 14878 & 67.65 & 13033 & 77.23 & 22470 \\
\hline 15758 & 71.34 & 13784 & 81.44 & 23765 \\
\hline 16643 & 74.80 & 14578 & 85.39 & 25135 \\
\hline 17614 & 77.90 & 15420 & 89.04 & 26002 \\
\hline 18609 & 81.84 & 16550 & 03.43 & 28185 \\
\hline 19872 & 86.38 & 17407 & 80.59 & 30011 \\
\hline 21002 & 9120 & 18507 & 104.12 & 31719 \\
\hline 22197 & 85.09 & 19444 & 108.56 & 33520 \\
\hline 23278 & 99.36 & 20300 & 113.43 & 35155 \\
\hline
\end{tabular}




\begin{tabular}{|c|c|c|c|c|}
\hline An (m) & $N(m)$ & Re 0 & Nu (0) & $\boldsymbol{R}_{\mathrm{m}}$ \\
\hline 24445 & 104.56 & 21413 & 119.37 & 30018 \\
\hline 20063 & 100.70 & 22471 & 124.19 & 88702 \\
\hline 20731 & 113.11 & 23415 & 128.13 & 40570 \\
\hline 20271 & 118.10 & 24764 & 134.82 & 42808 \\
\hline 20005 & 121.41 & 2547 & 138.60 & $45: 25$ \\
\hline 30114 & 125.25 & 26378 & 142.80 & 45470 \\
\hline 81287 & 120.50 & 27389 & 147.72 & 47221 \\
\hline 30618 & 136.74 & 20350 & 158.11 & 50020 \\
\hline 3458 & $140 . \pi$ & s0184 & 100.70 & 52040 \\
\hline 35001 & 143.50 & 31185 & 163.22 & 53768 \\
\hline 30026 & 146.83 & 32002 & 167.80 & 55314 \\
\hline 38700 & 151.85 & 30007 & 173.35 & 58460 \\
\hline 40002 & 156.54 & 35057 & 178.71 & 00442 \\
\hline 41673 & 161.95 & 36503 & 184.69 & 02036 \\
\hline 43423 & 168.15 & 38056 & 191.96 & 65579 \\
\hline 45343 & 174.08 & 39718 & 198.74 & 68479 \\
\hline 46014 & 178.83 & 41004 & 204.16 & 70851 \\
\hline 40267 & 186.33 & 43155 & 212.71 & 74405 \\
\hline 51336 & 183.04 & 44968 & 220.38 & 77530 \\
\hline 53606 & 200.14 & 46056 & 228.48 & 80058 \\
\hline 56136 & 210.01 & 49172 & 239.75 & 84778 \\
\hline 58369 & 216.86 & 51128 & 247.69 & 88151 \\
\hline 61558 & 224.78 & 53022 & 256.61 & 82068 \\
\hline 63781 & 232.06 & 55869 & 264.82 & 86324 \\
\hline 65616 & 237.88 & 57477 & 271.57 & 89097 \\
\hline 67457 & 244.10 & 59080 & 278.78 & 101876 \\
\hline $\cos 2$ & 252.10 & 61268 & 287.80 & 105620 \\
\hline 72364 & 250.53 & 63387 & 20823 & 109287 \\
\hline 74650 & 268.60 & 65397 & 304.36 & 112752 \\
\hline 77005 & 276.80 & 68240 & 316.22 & 117654 \\
\hline 79524 & 280.55 & 69659 & 320.28 & 120100 \\
\hline 80374 & 283.54 & 70404 & 323.70 & 121384 \\
\hline
\end{tabular}


Friction (D5, data set (2)): semicircular (radius $=4 \mathrm{~mm}$ ), $p / \theta=5, e / D=0.08, d_{m}=47.78 \mathrm{~mm}$ $\nabla_{A}(m)=0.995, \eta_{1}(m)=0.508, \downarrow_{A}(i)=0.998, \downarrow_{t}(0)=0.485$

\begin{tabular}{|c|c|c|c|c|c|}
\hline$n_{0}(m)$ & $f(m)$ & $\operatorname{Ro}(0)$ & 10 & $\mathbf{A n}_{m}$ & $t_{m}$ \\
\hline 1846 & 0.01974 & 1571 & 0.02068 & 1638 & 0.01003 \\
\hline 1722 & 0.01824 & 1644 & 0.02016 & 1714 & 0.00077 \\
\hline 1767 & 0.01883 & 1686 & 0.01973 & 1758 & 0.00057 \\
\hline 1817 & 0.01810 & 1735 & 0.01906 & 1800 & 0.00024 \\
\hline 1870 & 0.01773 & 1700 & 0.01858 & 1870 & 0.00001 \\
\hline 1830 & 0.01724 & 1851 & 0.01806 & 1850 & 0.00876 \\
\hline 1005 & 0.01678 & 1804 & 0.01756 & 1975 & 0.00852 \\
\hline 2005 & 0.01649 & 1943 & 0.01728 & 2026 & 0.00838 \\
\hline 2087 & 0.01650 & 1992 & 0.01738 & 2077 & 0.00843 \\
\hline 2130 & 0.01702 & 2033 & 0.01783 & 2120 & 0.00865 \\
\hline 2145 & 0.01758 & 2047 & 0.01842 & 2134 & 0.00893 \\
\hline 2167 & 0.01834 & 2068 & 0.01822 & 2156 & 0.00032 \\
\hline 2182 & 0.01975 & 2083 & 0.02070 & 2171 & 0.01003 \\
\hline 2176 & 0.02151 & 2077 & 0.002253 & 2166 & 0.01093 \\
\hline 2201 & $0.02 \times 28$ & 2100 & 0.02437 & 2180 & 0.01181 \\
\hline 2025 & 0.02550 & 2124 & 0.02671 & 2214 & 0.01285 \\
\hline 2202 & 0.02845 & 2150 & 0.02981 & 2250 & 0.01445 \\
\hline 2287 & 0.03130 & 2183 & 0.03279 & 2276 & 0.01590 \\
\hline 2314 & 0.03419 & 2200 & 0.03582 & 2303 & 0.01737 \\
\hline 2380 & 0.03710 & 2272 & 0.03887 & 2369 & 0.01885 \\
\hline 2407 & 0.04067 & 2097 & 0.04261 & 2385 & 0.02066 \\
\hline 2459 & 0.04383 & 2347 & 0.04552 & 2447 & 0.002227 \\
\hline 2551 & 0.04668 & 2435 & 0.04891 & 2538 & 0.02371 \\
\hline 2640 & 0.04903 & 2519 & 0.05199 & 2027 & 0.02521 \\
\hline 2771 & 0.06158 & 2845 & 0.05402 & 2758 & 0.02619 \\
\hline 2065 & 0.05344 & 2830 & 0.05599 & 2950 & 0.02715 \\
\hline 3150 & 0.05450 & 3007 & 0.05719 & 3135 & 0.02777 \\
\hline 3460 & 0.05628 & 3303 & 0.05897 & 3443 & 0.02859 \\
\hline 3870 & 0.05808 & 3504 & 0.06086 & 3851 & 0.02851 \\
\hline 4317 & 0.05858 & 4120 & 0.06138 & 4208 & 0.02076 \\
\hline 4857 & 0.06015 & 4638 & 0.06302 & 4833 & 0.03055 \\
\hline 5263 & 0.05015 & 50023 & 0.06302 & 5237 & 0.03055 \\
\hline
\end{tabular}




\begin{tabular}{|c|c|c|c|c|c|}
\hline$R_{0}(m)$ & $f(m)$ & P 0 & 10 & $\boldsymbol{R o}_{\mathrm{m}}$ & $t_{m}$ \\
\hline $\operatorname{sen} 2$ & 0.08081 & 8871 & 0.08372 & 8012 & 0.00080 \\
\hline 6702 & 0.00008 & 2007 & 0.06355 & 6800 & 0.05081 \\
\hline 7343 & 0.00051 & 7000 & 0.08320 & 73007 & 0.05074 \\
\hline$\infty$ & 0.00088 & 7886 & 0.08355 & 8221 & 0.05081 \\
\hline શ15 & 0.00000 & 8000 & 0.00006 & 8070 & 0.05053 \\
\hline œ10 & 0.05050 & 2050 & 0.00243 & 8061 & 0.05027 \\
\hline 10050 & 0.05028 & 10584 & 0.00211 & 10805 & 0.00011 \\
\hline 11857 & 0.05878 & 11317 & 0.06150 & 11700 & 0.02086 \\
\hline 12014 & 0.05858 & 12028 & 0.08117 & 12850 & 0.00066 \\
\hline 13002 & 0.05784 & 13845 & 0.05050 & 13013 & 0.06038 \\
\hline 14932 & 0.05760 & 14252 & 0.00044 & 14850 & 0.002831 \\
\hline 15866 & 0.05749 & 15143 & 0.00024 & 15787 & 0.02021 \\
\hline 16001 & 0.05711 & 16131 & 0.05083 & 16817 & 0.00001 \\
\hline 18127 & 0.05691 & 17301 & 0.05063 & 18037 & 0.02891 \\
\hline 18492 & 0.05672 & 18604 & 0.05042 & 19385 & 0.02881 \\
\hline 21558 & 0.05624 & 20576 & 0.05892 & 21452 & 0.02857 \\
\hline 23440 & 0.05657 & 22073 & 0.05927 & 20025 & 0.02874 \\
\hline 25904 & 0.05657 & 24724 & 0.05027 & 25776 & 0.02874 \\
\hline 28431 & 0.05001 & 27136 & 0.05863 & 20290 & 0.02801 \\
\hline 31769 & 0.05740 & 30522 & 0.00014 & 31612 & 0.02916 \\
\hline 36111 & 0.05774 & 34467 & 0.06049 & 35933 & 0.02933 \\
\hline 40455 & 0.05948 & 38613 & 0.06282 & 40256 & 0.03022 \\
\hline 44213 & 0.06076 & 42189 & 0.06356 & 43995 & 0.03087 \\
\hline 48984 & 0.06149 & 46753 & 0.06443 & 48743 & 0.03124 \\
\hline 53170 & 0.06384 & 50748 & 0.06688 & 52907 & 0.03243 \\
\hline 58158 & 0.06560 & 55500 & 0.08873 & 57871 & 0.003852 \\
\hline 61794 & 0.06701 & 58979 & 0.07020 & 61489 & 0.03404 \\
\hline 66163 & 0.06858 & 63150 & 0.07184 & 65837 & 0.08488 \\
\hline 71389 & 0.07022 & 68137 & 0.07357 & 71056 & 0.03567 \\
\hline 76633 & 0.07228 & 73142 & 0.07573 & 76254 & 0.03672 \\
\hline 80731 & 0.07472 & 77054 & 0.07828 & 80332 & 0.03785 \\
\hline 84831 & 0.07610 & 80567 & 0.07983 & 84412 & 0.03870 \\
\hline 86735 & 0.07671 & 82785 & 0.08037 & 60307 & 0.03897 \\
\hline
\end{tabular}


Heat transfer (D5, data set (2)): $\downarrow_{R}(0)=1.765, \downarrow_{R}(m)=1.684$

\begin{tabular}{|c|c|c|c|c|}
\hline Rs $(m)$ & Nu (m) & Ro 0 & Nu (0) & $\mathbf{R}_{\boldsymbol{o}_{m}}$ \\
\hline 1416 & 14.86 & 1351 & 15.56 & 2385 \\
\hline 1400 & 16.30 & 1422 & 16.03 & 2510 \\
\hline 1570 & 15.70 & 1507 & 16.54 & 2660 \\
\hline 1670 & 16.24 & 1500 & 17.02 & 2021 \\
\hline 1702 & 16.73 & 1681 & 17.53 & 2067 \\
\hline 1849 & 1721 & 1764 & 18.03 & 3114 \\
\hline 1843 & 17.80 & 1854 & 18.75 & 9272 \\
\hline 2059 & 18.58 & 1865 & 10.47 & 3468 \\
\hline 2193 & 18.36 & 2003 & 20.28 & 3094 \\
\hline 2050 & 20.11 & 2024 & 21.07 & 3924 \\
\hline 2452 & 21.06 & 2340 & 22.07 & 4120 \\
\hline 2526 & 21.71 & 2411 & 22.75 & 4254 \\
\hline 2615 & 22.37 & 2496 & 23.44 & 4405 \\
\hline 274 & 23.22 & 2619 & 24.33 & 4022 \\
\hline 2868 & 2420 & 2737 & 25.35 & 4830 \\
\hline 2942 & 24.73 & 2808 & 25.91 & 4955 \\
\hline 3070 & 25.63 & 2230 & 26.85 & 5170 \\
\hline 3247 & 28.68 & 3000 & 27.85 & 5468 \\
\hline 3538 & 28.88 & 3377 & 30.26 & 5958 \\
\hline 3736 & 30.70 & 3566 & 32.16 & 6283 \\
\hline 3046 & 32.46 & 3766 & 34.01 & 6846 \\
\hline 4188 & 34.00 & 3998 & 35.71 & 7054 \\
\hline 4424 & 36.15 & 4202 & 37.88 & 7450 \\
\hline 4760 & 38.45 & 4542 & 40.28 & 8015 \\
\hline 5050 & 40.88 & 4800 & 4281 & 8471 \\
\hline 8360 & 43.03 & 5108 & 45.08 & 8010 \\
\hline 5808 & 44.98 & 5381 & 47.10 & 9498 \\
\hline 5033 & 47.34 & 5663 & 49.60 & 9993 \\
\hline 6370 & 80.50 & 6079 & 52.80 & 10728 \\
\hline 6781 & 52.84 & 6453 & 55.38 & 11387 \\
\hline 7171 & 55.56 & 6244 & 58.21 & 12077 \\
\hline
\end{tabular}




\begin{tabular}{|c|c|c|c|c|}
\hline$P(m)$ & $\operatorname{Mu}(\mathrm{m})$ & $\boldsymbol{A}(1)$ & Nu (1) & $\mathbf{P}_{\mathrm{m}}$ \\
\hline 7008 & 68.18 & 7203 & $\infty .08$ & 12746 \\
\hline $\cos 5$ & 6.53 & 7088 & 03.42 & 13657 \\
\hline 2405 & 0240 & 0003 & 65.47 & 14157 \\
\hline$\infty 000$ & 06.43 & 6552 & 68.56 & 15000 \\
\hline e4to & 68.47 & $\infty 010$ & 71.73 & 10015 \\
\hline e:25 & 71.08 & esro & 74.48 & 16848 \\
\hline 10347 & 74.17 & ea76 & 77.71 & 17427 \\
\hline 11045 & 78.21 & 10042 & 81.85 & 18000 \\
\hline 11731 & 81.67 & 11197 & 85.56 & 18750 \\
\hline 12520 & 84.31 & 11750 & 88.33 & 20750 \\
\hline 13188 & 88.53 & 12587 & 2276 & 2212 \\
\hline 13978 & 82.80 & 13341 & 97.33 & 23542 \\
\hline 14878 & 9727 & 14201 & 101.82 & 25050 \\
\hline 15492 & 101.35 & 14787 & 106.18 & 26003 \\
\hline 16143 & 108.53 & 15408 & 108.47 & 27188 \\
\hline 16809 & 108.48 & 16215 & 113.66 & 28613 \\
\hline 17081 & 113.51 & 17162 & 118.82 & 30204 \\
\hline 18842 & 117.85 & 17884 & 120.47 & 31735 \\
\hline 19014 & 122.79 & 18007 & 128.65 & 85541 \\
\hline 21550 & 129.94 & 20550 & 136.15 & 30203 \\
\hline 22068 & 135.87 & 21827 & 142.36 & 38516 \\
\hline 24324 & 141.87 & 25216 & 148.64 & 40968 \\
\hline 26168 & 140.30 & 24976 & 156.52 & 44073 \\
\hline 27794 & 155.88 & 26528 & 163.32 & 46812 \\
\hline 29668 & 167.08 & 28317 & 175.05 & 49969 \\
\hline 31312 & 17421 & 29883 & 182.52 & 52737 \\
\hline 32952 & 18125 & 31452 & 189.90 & 55500 \\
\hline 34852 & 180.52 & 35264 & 188.56 & 58690 \\
\hline $\mathbf{3 6 7 3 0}$ & 186.91 & 35057 & 206.30 & 61829 \\
\hline 38572 & 204.87 & 38816 & 214.65 & 64958 \\
\hline 40911 & 214.97 & 30048 & 224.60 & 68005 \\
\hline 44012 & 227.18 & 42008 & 238.02 & 74128 \\
\hline 46681 & 241.27 & 44555 & 252.79 & 78623 \\
\hline
\end{tabular}




\begin{tabular}{|c|c|c|c|c|}
\hline Do (m) & Nu (m) & Ro 0 & Nu $(1)$ & $R_{m}$ \\
\hline 48007 & 200.14 & 46004 & 202.08 & 81867 \\
\hline 80027 & 207.88 & 48005 & 270.18 & 84763 \\
\hline 80006 & 268.12 & 40031 & 200.91 & 88188 \\
\hline 64527 & 278.78 & 82043 & 202.07 & 91837 \\
\hline 68415 & 286.7 & $5 \operatorname{sen} 6$ & 500.48 & $\mathscr{9 0 1 7}$ \\
\hline 60751 & $\mathbf{5 0 0 . 4 0}$ & 57050 & 314.83 & 1000337 \\
\hline 08345 & 315.88 & 60747 & 331.08 & 107195 \\
\hline 67840 & 322.51 & 64568 & 348.38 & 113038 \\
\hline 60500 & 343.78 & $e 8144$ & 900.17 & 116719 \\
\hline 72006 & 854.80 & 08726 & 571.83 & 121276 \\
\hline 75083 & 308.72 & 71664 & 386.32 & 123450 \\
\hline 76753 & 375.31 & 70257 & 303.22 & 120271 \\
\hline 78126 & 382.01 & 74568 & 400.24 & 131584 \\
\hline
\end{tabular}

Friction (D10, data set (1)): $p / e=10, e / D=0.08, d_{m}=48.92 \mathrm{~mm}$ $\downarrow_{R}(m)=1.241, \downarrow_{1}(m)=0.427, \downarrow_{R}(0)=1.270, \downarrow_{1}(m)=0.417$

\begin{tabular}{|c|c|c|c|c|c|}
\hline$R_{0}(m)$ & $f(m)$ & Re (D) & 10 & $\mathbf{R}_{m}$ & $t_{m}$ \\
\hline 503 & 0.04963 & 579 & 0.05078 & 736 & 0.02119 \\
\hline 623 & 0.04780 & 600 & 0.04871 & 774 & 0.02032 \\
\hline 651 & 0.04554 & 636 & 0.04660 & 808 & 0.01944 \\
\hline 680 & 0.04302 & 674 & 0.04402 & 856 & 0.01837 \\
\hline 720 & 0.04084 & 704 & 0.04180 & 804 & 0.01744 \\
\hline 753 & 0.03941 & 736 & 0.04033 & 825 & 0.01683 \\
\hline 784 & 0.03806 & 766 & 0.03895 & 973 & 0.01625 \\
\hline 814 & 0.05675 & 796 & 0.03761 & $1011_{1}$ & 0.01560 \\
\hline 845 & 0.03571 & 828 & 0.03654 & 1049 & 0.01524 \\
\hline 873 & 0.03466 & 853 & 0.03547 & 1083 & 0.01460 \\
\hline 907 & 0.03027 & 886 & 0.03405 & 1128 & 0.01421 \\
\hline 845 & 0.05200 & 823 & 0.05274 & 1173 & 0.01366 \\
\hline 970 & 0.00100 & 857 & 0.03175 & 1216 & 0.01325 \\
\hline 1010 & 0.03007 & 987 & 0.03077 & 1254 & 0.01284 \\
\hline 1038 & 0.02019 & 1014 & 0.02087 & 1288 & 0.01246 \\
\hline
\end{tabular}




\begin{tabular}{|c|c|c|c|c|c|}
\hline$R(m)$ & $f(m)$ & Po 0 & 10 & $\mathbf{A m}_{m}$ & $t_{m}$ \\
\hline 1102 & 0.02768 & 1077 & 0.002831 & 1398 & 0.01181 \\
\hline 1135 & 0.02002 & 1100 & 0.02755 & 1400 & 0.01140 \\
\hline 1163 & $0.005 \times 5$ & 1136 & 0.00697 & 1443 & 0.01125 \\
\hline 1180 & 0.00558 & 1172 & 0.02816 & 1488 & 0.01001 \\
\hline 1240 & 0.02487 & 1211 & 0.000545 & 1500 & 0.01082 \\
\hline 1204 & $0.00 \times 98$ & 1284 & 0.02454 & 1608 & 0.01024 \\
\hline 1300 & 0.00200 & 1308 & $0.00 \times 74$ & 1602 & 0.00090 \\
\hline 1418 & 0.00200 & 1384 & 0.00258 & 1758 & 0.00942 \\
\hline 1483 & 0.02113 & 1440 & 0.02162 & 1841 & 0.00502 \\
\hline 1544 & 0.00028 & 1800 & 0.00074 & 1917 & 0.00885 \\
\hline 1600 & 0.01974 & 1584 & 0.00020 & 1837 & 0.00843 \\
\hline 1653 & 0.01875 & 1615 & 0.02021 & 2052 & 0.00843 \\
\hline 1609 & 0.01898 & 1000 & 0.02046 & 2109 & 0.00253 \\
\hline 1727 & 0.02073 & 1687 & 0.02122 & 2143 & 0.00885 \\
\hline 1738 & 0.02165 & 1000 & 0.02216 & 2158 & 0.00024 \\
\hline 1741 & 0.02267 & 1702 & 0.02320 & 2162 & 0.00968 \\
\hline 1774 & 0.00354 & 1734 & 0.02408 & 2203 & 0.01005 \\
\hline 1787 & 0.02500 & 1746 & 0.02558 & 2218 & 0.01067 \\
\hline 1780 & 0.02727 & 1758 & 0.02780 & 2233 & 0.01164 \\
\hline 1814 & 0.05043 & 1773 & 0.00114 & 2252 & 0.01299 \\
\hline 1842 & 0.05373 & 1800 & 0.03451 & 2287 & 0.01440 \\
\hline 1868 & 0.08685 & 1825 & 0.03771 & 2318 & 0.01573 \\
\hline 1890 & 0.04053 & 1847 & 0.04127 & 2347 & 0.01722 \\
\hline 1918 & 0.04447 & 1874 & 0.04551 & 2381 & 0.01899 \\
\hline 1943 & 0.04858 & 1698 & 0.04972 & 2412: & 0.02074 \\
\hline 1978 & 0.05855 & 1033 & 0.05450 & 2455 & 0.02278 \\
\hline 2002 & 0.05784 & 1956 & 0.05919 & 2485 & 0.02469 \\
\hline 2005 & 0.05035 & 1950 & 0.08176 & 2469 & 0.02577 \\
\hline 2034 & 0.06411 & 1888. & 0.06580 & 2525 & 0.02737 \\
\hline 2112 & 0.06962 & 2084 & 0.07124 & 2622 & 0.02872 \\
\hline 2162 & 0.07384 & 2112 & 0.07538 & 2883 & 0.03144 \\
\hline 2279 & 0.07819 & 2027 & 0.07797 & 2820 & 0.05253 \\
\hline 2417 & 0.07816 & $23 \approx 2$ & 0.07998 & 5001 & 0.03337 \\
\hline
\end{tabular}




\begin{tabular}{|c|c|c|c|c|c|}
\hline Po (m) & $f(m)$ & Ro $(0)$ & 10 & $\mathbf{P a s}_{m}$ & $t_{m}$ \\
\hline 207 & 0.07700 & 2510 & 0.07971 & 3180 & 0.03826 \\
\hline 2000 & 0.07816 & 2745 & 0.07898 & 3488 & 0.03335 \\
\hline 3052 & 0.07816 & 2033 & 0.07998 & 3789 & 0.03337 \\
\hline 505 & $0.077 \times 3$ & 3191 & 0.07878 & 4053 & 0.03528 \\
\hline 3481 & 0.07600 & 8402 & 0.07095 & 4522 & 0.03831 \\
\hline 9024 & 0.07783 & 8541 & 0.07894 & 498 & 0.03323 \\
\hline 3772 & 0.07743 & 3686 & 0.07924 & 4683 & 0.03506 \\
\hline 4101 & 0.07763 & 1008 & 0.07944 & $\sec 2$ & 0.03014 \\
\hline 4437 & 0.07750 & 4356 & 0.07030 & 8503 & 0.03300 \\
\hline 4004 & 0.0778 & 4587 & 0.07964 & 5827 & 0.03323 \\
\hline 5086 & 0.07750 & 4971 & 0.07930 & 6314 & 0.03500 \\
\hline 5143 & 0.07743 & 5026 & 0.07924 & 6384 & 0.03506 \\
\hline 5414 & 0.07717 & 5000 & 0.07897 & 672) & 0.03295 \\
\hline 5737 & 0.07704 & 6007 & 0.07883 & $712 ?$ & 0.03280 \\
\hline 6054 & 0.07652 & 5017 & 0.07830 & 7516 & 0.03267 \\
\hline 6449 & 0.07665 & 6003 & 0.07843 & 8005 & 0.03272 \\
\hline 6847 & 0.07645 & 6091 & 0.07823 & 8500 & 0.03264 \\
\hline 7293 & 0.07632 & 7127 & 0.07810 & 8054 & 0.03258 \\
\hline$\pi / 23$ & 0.07555 & 7547 & 0.07731 & 2587 & 0.05225 \\
\hline 8097 & 0.07542 & 8108 & 0.07718 & 10301 & 0.03220 \\
\hline 8037 & 0.07516 & 8734 & 0.07691 & 11006 & 0.03200 \\
\hline 9693 & 0.07491 & 8472 & 0.07665 & 12038 & 0.03198 \\
\hline 10176 & 0.07472 & 8944 & 0.07646 & 12639 & 0.03190 \\
\hline 10657 & 0.07415 & 10414 & 0.07587 & 13225 & 0.03166 \\
\hline 11449 & 0.07421 & 11188 & 0.07594 & 14213 & 0.03168 \\
\hline 12652 & 0.07427 & 12364 & 0.07600 & 15707 & 0.03171 \\
\hline 13535 & 0.07384 & 13027 & 0.07536 & 16000 & 0.03144 \\
\hline 14350 & 0.07358 & 14042 & 0.07529 & 17838 & 0.03141 \\
\hline 15372 & 0.07427 & 15002 & 0.07600 & 19080 & 0.03171 \\
\hline 16057 & 0.07389 & 15691 & 0.07562 & 19933 & 0.03155 \\
\hline 17163 & 0.07389 & 16772 & 0.07562 & 21306 & 0.03155 \\
\hline 18645 & 0.07472 & 18220 & 0.07646 & 23146 & 0.03190 \\
\hline 19709 & 0.07465 & 18260 & 0.07639 & 24467 & 0.03187 \\
\hline
\end{tabular}




\begin{tabular}{|c|c|c|c|c|c|}
\hline no $(m)$ & $f(m)$ & Po 0 & 10 & $R \boldsymbol{o}_{m}$ & $t_{m}$ \\
\hline 21505 & 0.07446 & 21103 & 0.07620 & 26808 & 0.03179 \\
\hline 23420 & 0.07478 & 22887 & 0.07852 & 20074 & 0.03193 \\
\hline 25405 & 0.07465 & 24885 & 0.07630 & 31612 & 0.03187 \\
\hline 27504 & 0.07516 & 20065 & 0.07691 & 34255 & 0.03200 \\
\hline 220219 & 0.07548 & 28553 & 0.07724 & 30272 & 0.002023 \\
\hline 31631 & 0.07000 & 30040 & $0.07 m$ & 30304 & 0.03245 \\
\hline 39087 & 0.07008 & 89213 & 0.07783 & 42122 & 0.03247 \\
\hline 38010 & 0.07806 & $\$ 7144$ & 0.07783 & 47186: & 0.05247 \\
\hline 40214 & 0.07801 & 50003 & 0.07870 & 40002 & 0.03283 \\
\hline 49168 & 0.07710 & 42185 & 0.07890 & 53580 & 0.03292 \\
\hline 46181 & 0.07737 & 45130 & 0.07917 & 57330 & 0.03303 \\
\hline 49701 & 0.07750 & 48560 & 0.07930 & 61609. & 0.03509 \\
\hline 53626 & 0.07820 & 52405 & 0.08012 & 66572 & 0.03343 \\
\hline 57027 & 0.07840 & 55728 & 0.08032 & 70794 & 0.03351 \\
\hline 60799 & 0.07943 & 59415 & 0.08129 & $754 \pi$ & 0.03391 \\
\hline 66447 & 0.07991 & 64933 & 0.08177 & 82488 & 0.03412 \\
\hline 71817 & 0.00050 & 70181 & 0.08247 & 69154 & 0.03441 \\
\hline 78554 & 0.08011 & 76766 & 0.08188 & 97518 & 0.03420 \\
\hline 83252 & 0.08052 & 81356 & 0.08240 & 103349 & 0.03438 \\
\hline
\end{tabular}

Heat transfer (D10, data set (1)): $\downarrow_{A}(0)=2.668, \downarrow_{R}(r n)=2.607$

\begin{tabular}{|c|c|c|c|c|}
\hline$B e(m)$ & $\mathrm{Nu}(\mathrm{m})$ & Pa (1) & Nu (i) & $\mathbf{R o}_{\mathrm{m}}$ \\
\hline 508 & 7.30 & 497 & 7.47 & 1325 \\
\hline 520 & 7.61 & . $\quad 517$ & 7.79 & 1380 \\
\hline 861 & 8.00 & 549 & 8.18 & 1464 \\
\hline 508 & 8.48 & 582 & 8.68 & 1553 \\
\hline 647 & 9.07 & 682 & 9.28 & 1685 \\
\hline 694 & 0.62 & 678 & 9.84 & 1800 \\
\hline 752 & 10.20 & 735 & 10.53 & 1800 \\
\hline 810 & 10.87 & 782 & 11.12 & 2112 \\
\hline 867 & 11.38 & 847 & 11.64 & 2250 \\
\hline 922 & 11.88 & 801 & 12.15 & 2405 \\
\hline
\end{tabular}




\begin{tabular}{|c|c|c|c|c|}
\hline$P_{0}(m)$ & Nu (m) & Ro (1) & Nu (1) & $F \boldsymbol{w}_{m}$ \\
\hline ers & 12.31 & 055 & 12.50 & 2549 \\
\hline 1058 & 12.70 & 1014 & 13.00 & 2705 \\
\hline 1007 & 13.24 & 1072 & 13.55 & 2850 \\
\hline 1150 & 13.58 & 1124 & 13.85 & 2998 \\
\hline 1200 & 13.89 & 1182 & 14.31 & 9180 \\
\hline 1301 & 14.52 & 1272 & 14.88 & 3392 \\
\hline 1380 & 15.05 & 1340 & 15.40 & 3508 \\
\hline 1451 & 18.56 & 1418 & 15.82 & 3784 \\
\hline 1617 & 16.08 & 1482 & 16.46 & 3054 \\
\hline 1620 & 17.07 & 1583 & 17.47 & 4223 \\
\hline 1609 & 17.85 & 1660 & 18.26 & 4420 \\
\hline 1775 & 18.78 & 1735 & 18.22 & 4628 \\
\hline 1850 & 19.66 & 1808 & 20.12 & 4822 \\
\hline 1836 & 20.44 & 1802 & 20.82 & 5046 \\
\hline 2029 & 21.51 & 1882 & 22.02 & 5288 \\
\hline 2124 & 22.38 & 2076 & 22.01 & 5538 \\
\hline 2256 & 23.79 & 2205 & 24.34 & 5882 \\
\hline 2353 & 24.82 & 2009 & 25.40 & 6133 \\
\hline 2504 & 26.14 & 2447 & 26.75 & 6528 \\
\hline 2647 & 27.53 & 2587 & 28.17 & 6000 \\
\hline 2825 & 29.07 & 2761 & 29.74 & 7365 \\
\hline 2909 & 30.94 & 2031 & 31.66 & 7817 \\
\hline 3254 & 32.90 & 3180 & 33.67 & 8482 \\
\hline 8525 & 35.22 & 3445 & 36.04 & 9180 \\
\hline 3739 & 37.24 & 3654 & 38.11 & 9747 \\
\hline 3969 & 38.94 & 3878 & 39.85 & 10345 \\
\hline 4150 & 40.60 & 4064 & 41.55 & 10041 \\
\hline 4430 & 49.30 & 4388 & 44.31 & 11572 \\
\hline 4793 & 46.42 & 4684 & 4750 & 12494 \\
\hline 5182 & 49,61 & 5064 & 50.7 & 13507 \\
\hline 5360 & 51.20 & 5246 & 52.48 & 13085 \\
\hline 5702 & 63.82 & 5572 & 55.07 & 14865 \\
\hline 6161 & 57.40 & 6020 & 58.74 & 16000 \\
\hline
\end{tabular}




\begin{tabular}{|c|c|c|c|c|}
\hline Po (m) & $N w(m)$ & R日 (1) & Nu (1) & $\boldsymbol{P}_{m}$ \\
\hline cose & $\infty .74$ & eA81 & 02.18 & 17280 \\
\hline 7145 & 03.02 & $\cos$ & 6.41 & 18028 \\
\hline 7085 & 67.36 & 7400 & 68.83 & 10081 \\
\hline 8248 & 71.64 & 8058 & 73.31 & 21406 \\
\hline exas & 76.76 & 8731 & 7.60 & 20000 \\
\hline 8071 & 70.78 & 0353 & 81.84 & 24940 \\
\hline 10187 & 83.71 & $\cos 5$ & 85.67 & 26558 \\
\hline 10888 & 88.00 & 10819 & $\infty .15$ & 28326 \\
\hline 11411 & 91.72 & 11161 & 03.88 & 20747 \\
\hline 12204 & 96.65 & 12014 & $\mathscr{e 8 . 8 1}$ & 32047 \\
\hline 13310 & 103.38 & 13007 & 105.79 & 34097 \\
\hline 14360 & 110.42 & 14033 & 112.99 & 37433 \\
\hline 15304 & 115.86 & 15043 & 118.56 & 40128 \\
\hline 16525 & 122.62 & 16149 & 125.47 & 40078 \\
\hline 17841 & 120.30 & 17435 & 132.41 & 46507 \\
\hline 19454 & 137.13 & 18011 & 140.32 & 50711 \\
\hline 21182 & 146.36 & 20700 & $149 . \pi$ & 55217 \\
\hline 22435 & 152.83 & 21924 & 156.49 & 58482 \\
\hline 23914 & 161.85 & 23360 & 165.73 & 62338 \\
\hline 25400 & 169.10 & 24821 & 173.04 & 66212 \\
\hline 26769 & 176.07 & 23150 & 180.17 & 69780 \\
\hline 28392 & 162.83 & 27745 & 187.10 & 74012 \\
\hline 29668 & 191.14 & 28993 & 185.50 & 77339 \\
\hline 29480 & 191.00 & 28808 & 185.45 & 76847 \\
\hline 31245 & 188.16 & 30534 & 202.78 & 81450 \\
\hline 32768 & 204.72 & 32020 & 200.50 & 85414 \\
\hline 33328 & 200.42 & 32569 & 214.30 & 86881 \\
\hline 36110. & 221.15 & 35288 & 226.30 & 84131 \\
\hline 36110 & 222.72 & 35288 & 227.91 & 94131 \\
\hline 38137 & 234.54 & 37260 & 240.00 & $\$ 9416$ \\
\hline 40421 & 245.58 & 30501 & 251.31 & 105370 \\
\hline 43208 & 257.70 & 42224 & 253.70 & 112635 \\
\hline 46122 & 271.17 & 45072 & 277.49 & 120200 \\
\hline
\end{tabular}




\begin{tabular}{|c|c|c|c|c|}
\hline Po $(m)$ & $N u(m)$ & Ro 0 & $\mathrm{Nu}(\mathrm{O}$ & $\mathbf{R}_{m}$ \\
\hline 48746 & 205.78 & 47855 & 20242 & 127070 \\
\hline 51203 & 208.60 & 50008 & 803.60 & 135634 \\
\hline $5 \sec$ & 207.81 & 52460 & 314.80 & 139989 \\
\hline 65818 & 316.88 & 54547 & 32426 & 145500 \\
\hline $\operatorname{se2} 45$ & 20023 & 50019 & 358.50 & 151833 \\
\hline 01897 & 343.78 & 60585 & 351.77 & 161613 \\
\hline 65570 & 850.10 & 64077 & 367.56 & 170027 \\
\hline 68572 & 87292 & 66815 & 881.81 & 178233 \\
\hline 71042 & 305.00 & 60424 & 303.97 & 186192 \\
\hline 75859 & 30521 & 71882 & 404.42 & 182014 \\
\hline 74711 & 400.28 & 73010 & 409.61 & 194758 \\
\hline
\end{tabular}

Friction (D20, data set (3)): $p / e=20, e / D=0.08, d_{m}=49.50 \mathrm{~mm}$ $\nabla_{A}(m)=2.032, \downarrow_{1}(m)=0.241, \downarrow_{R}(i)=2.055, \downarrow_{1}(i)=0.238$

\begin{tabular}{|c|c|c|c|c|c|}
\hline$R \theta(m)$ & $f(m)$ & $R 00$ & $f(0)$ & $\mathbf{P o}_{\mathrm{m}}$ & $t_{m}$ \\
\hline 628 & 0.05501 & 621 & 0.05563 & 1276 & 0.01324 \\
\hline 656 & 0.05294 & 648 & 0.05354 & 1382 & 0.01274 \\
\hline 682 & 0.05044 & 675 & 0.05101 & 1387 & 0.01214 \\
\hline 705 & 0.04912 & $\infty 97$ & 0.04968 & 1432 & 0.01182 \\
\hline 731 & 0.04672 & 723 & 0.04725 & 1486 & 0.01124 \\
\hline 755 & 0.04531 & 747 & 0.04582 & 1535 & 0.01000 \\
\hline 782 & 0.04391 & 773 & 0.04440 & 1588 & 0.01056 \\
\hline 820 & 0.04215 & 811 & 0.04263 & 1666 & 0.01014 \\
\hline 858 & 0.04015 & 848 & 0.04061 & 1743 & 0.00066 \\
\hline 876 & 0.03934 & 867 & 0.03979 & 1781 & 0.00947 \\
\hline 8003 & 0.03816 & 803 & 0.03850 & 1835. & 0.00018 \\
\hline $\cos$ & 0.03710 & 222 & 0.03752 & 1804 & 0.00893 \\
\hline 858 & 0.03595 & 847 & 0.03636 & 1846 & 0.00865 \\
\hline$\infty 80$ & 0.03478 & 870 & 0.03517 & 2012 & 0.00837 \\
\hline 1018 & 0.03460 & 1006 & 0.03499 & 2068 & 0.00833 \\
\hline 1044 & 0.03454 & 1032 & 0.03493 & 2122 & 0.00831 \\
\hline 1076 & 0.03525 & 1064 & 0.03565 & 2188 & 0.00848 \\
\hline 1114 & 0.05568 & 1102 & 0.03708 & 2264 & 0.00882 \\
\hline
\end{tabular}




\begin{tabular}{|c|c|c|c|c|c|}
\hline$n_{0}(m)$ & $f(m)$ & $R 0$ & 10 & $\mathbf{A o}_{\mathrm{m}}$ & $t_{m}$ \\
\hline 1158 & 0.08812 & 1140 & 0.08856 & 2043 & 0.00017 \\
\hline 1105 & 0.04019 & 1182 & 0.04084 & 2128 & 0.00967 \\
\hline 1245 & 0.04205 & 1231 & 0.04343 & 2500 & 0.01033 \\
\hline 1200 & 0.04512 & 1276 & 0.04563 & 2002 & 0.01086 \\
\hline 1342 & 0.04802 & 1827 & 0.04017 & 2727 & 0.01170 \\
\hline 1416 & 0.06152 & 1400 & 0.05210 & 2878 & 0.01240 \\
\hline 1486 & 0.05385 & 1400 & 0.05446 & 3019 & 0.01206 \\
\hline 1503 & 0.05653 & 1576 & 0.05716 & 5238 & 0.01360 \\
\hline 1699 & 0.05804 & 1680 & 0.05869 & 3452 & 0.01306 \\
\hline 1833 & 0.05888 & 1813 & 0.05855 & 3725 & 0.01417 \\
\hline 1883 & 0.05028 & 1861 & 0.05095 & 10000 & 0.01426 \\
\hline 2178 & 0.05018 & 2154 & 0.05085 & 426 & 0.01424 \\
\hline 2099 & 0.05898 & 2372 & 0.05965 & 4875 & 0.01418 \\
\hline 2681 & 0.05918 & 2651 & 0.05985 & 5447 & 0.01424 \\
\hline 2945 & 0.05878 & 2012 & 0.05045 & 5083 & 0.01414 \\
\hline 3218 & 0.05813 & 3182 & 0.05879 & 6539 & 0.01399 \\
\hline 3307 & 0.05813 & 3270 & 0.05879 & 6720 & 0.01309 \\
\hline 3499 & 0.05780 & 3460 & 0.05854 & 7110 & 0.01303 \\
\hline 3027 & 0.05779 & 3784 & 0.05844 & $7 m$ & 0.01390 \\
\hline 4087 & 0.05813 & 4042 & 0.05879 & 8306 & 0.01300 \\
\hline 440 & 0.05759 & 4091 & 0.05824 & 8023 & 0.01386 \\
\hline 4832 & 0.05735 & 4778 & 0.05800 & 8919 & 0.01380 \\
\hline 5281 & 0.05686 & 5022 & 0.05751 & 10731 & 0.01368 \\
\hline 5932 & 0.05667 & 5865 & 0.05731 & 12053 & 0.01364 \\
\hline 6490 & 0.05653 & 6426 & 0.05716 & 13206 & $0.013 \times 0$ \\
\hline 7012 & 0.05633 & 6034 & 0.05697 & 14249 & 0.01355 \\
\hline 7540 & 0.05628 & 7458 & 0.05602 & 15322 & 0.01354 \\
\hline 8248 & 0.05581 & 8156 & 0.05644 & 16760 & 0.01345 \\
\hline 8115 & 0.05581 & 8013 & 0.05844 & 18521 & 0.01348 \\
\hline 9944 & 0.05582 & 8833 & 0.05005 & 20207 & 0.01358 \\
\hline 10089 & 0.05557 & 10868 & $0.05 \times 200$ & 22330 & 0.01337 \\
\hline 12031 & 0.05843 & 11896 & 0.05808 & 24446 & 0.01334 \\
\hline 13520 & 0.05543 & 13180 & 0.05606 & 27084 & 0.01334 \\
\hline
\end{tabular}




\begin{tabular}{|c|c|c|c|c|c|}
\hline Do $(m)$ & $f(m)$ & no 0 & 10 & $\mathrm{Ao}_{\mathrm{m}}$ & $t_{m}$ \\
\hline 14504 & 0.08557 & 14203 & 0.05820 & 20248 & 0.01337 \\
\hline 15833 & 0.05802 & 15161 & 0.05025 & $\$ 1156$ & 0.01358 \\
\hline 16200 & 0.05581 & 160027 & 0.05644 & 52035 & 0.01343 \\
\hline 17017 & 0.05571 & 16826 & 0.05634 & 34578 & 0.01341 \\
\hline 18376 & $0.05=82$ & 18171 & 0.05625 & 37340 & 0.01338 \\
\hline 10350 & 0.05681 & 10142 & 0.05644 & 30337 & 0.01343 \\
\hline 20657 & 0.05624 & 20128 & 0.05687 & 41975 & 0.01353 \\
\hline 22027 & 0.05624 & 2007 & 0.05687 & 45568 & 0.01353 \\
\hline 23865 & 0.05628 & 25508 & 0.05092 & 48493 & 0.01354 \\
\hline 25443 & 0.05828 & 205150 & 0.05692 & 51701 & 0.01354 \\
\hline 20965 & 0.05614 & 26663 & 0.05678 & 54782 & 0.01351 \\
\hline 29621 & 0.05638 & 29289 & 0.05702 & 60189 & 0.01357 \\
\hline 31832 & 0.05677 & 31575 & 0.05741 & 64887 & 0.01366 \\
\hline 34750 & 0.05730 & 34361 & 0.05785 & 70612 & 0.01379 \\
\hline 37816 & 0.05730 & 37393 & 0.05795 & 76842 & 0.01379 \\
\hline 40008 & 0.05706 & 40242 & 0.05770 & 82098 & 0.01373 \\
\hline 43949 & 0.05677 & 43458 & 0.05741 & 89305 & 0.01366 \\
\hline 47178 & 0.05725 & 46850 & 0.05790 & 95866 & 0.01378 \\
\hline 50091 & 0.05725 & 50420 & 0.05790 & 103613 & 0.01378 \\
\hline 56061 & 0.05779 & 55434 & 0.05844 & 113917 & 0.01390 \\
\hline 50211 & 0.05779 & 58548 & 0.05844 & 120316 & 0.01390 \\
\hline 62644 & 0.05780 & 61943 & 0.05854 & 127293 & 0.01303 \\
\hline 66107 & 0.05789 & 65367 & 0.05854 & 134330 & 0.01393 \\
\hline 70063 & 0.05808 & 70169 & 0.05874 & 144198 & 0.01398 \\
\hline 74249 & 0.05804 & 73418 & 0.05869 & 150875 & 0.01396 \\
\hline 76081 & 0.05204 & 76100 & 0.05869 & 156385 & 0.01398 \\
\hline 78490 & 0.05853 & 78610 & 0.05918 & 161543 & 0.01408 \\
\hline
\end{tabular}


Heat transfer (D20, data set $(3)): \downarrow_{A}(m)=2.607, \downarrow_{A}(I)=2.668$

\begin{tabular}{|c|c|c|c|c|}
\hline $\mathrm{Ao}_{0}(\mathrm{~m})$ & Nu (m) & An 0 & Nu 10 & $\mathbf{R o}_{m}$ \\
\hline $\operatorname{exs}$ & 10.84 & 897 & 10.76 & 2004 \\
\hline ex4 & 11.91 & 2053 & 1123 & 2543 \\
\hline 1010 & 11.40 & $\infty$ & 11.62 & 2684 \\
\hline 1000 & 11.84 & 1050 & 11.97 & 2770 \\
\hline 1104 & 1222 & 1001 & 12.36 & 2012 \\
\hline 1150 & 1270 & 1137 & 12.85 & 30034 \\
\hline 1206 & 13.12 & 1183 & 13.27 & 3181 \\
\hline 1284 & 13.64 & 1250 & 13.79 & 3354 \\
\hline 1302 & 14.14 & 1307 & 14.30 & 3486 \\
\hline 1389 & 14.60 & 1373 & 14.85 & 3664 \\
\hline 1477 & 15.46 & 1461 & 15.63 & 3897 \\
\hline 1555 & 16.08 & 1537 & 16.26 & 4101 \\
\hline 1670 & 17.15 & 1652 & 17.35 & 4406 \\
\hline 1775 & 18.14 & 1756 & 18.34 & 4683 \\
\hline 1774 & 18.15 & 1754 & 18.36 & 4680 \\
\hline 1845 & 18.85 & 1824 & 19.06 & 4866 \\
\hline 2017 & 20.53 & 1005 & 20.76 & 5221 \\
\hline 2231 & 22.37 & 2006 & 22.02 & 5825 \\
\hline 2002 & 23.86 & 2365 & 24.13 & 6308 \\
\hline 2547 & 25.12 & 2519 & 25.41 & 6719 \\
\hline 2756 & 28.85 & 2725 & 27.25 & 7270 \\
\hline 5037 & 20.07 & 3003 & 20.40 & 8012 \\
\hline 8247 & 30.74 & 3210 & 31.00 & 8564 \\
\hline 3481 & 32.51 & 3442 & 32.88 & 9181 \\
\hline 3742 & 34.33 & 3700 & 34.72 & 9869 \\
\hline 8007 & 35.34 & 3860 & 55.74 & 10306 \\
\hline 4050 & 38.72 & 4014 & 37.13 & 10708 \\
\hline 4200 & 87.72 & 4162 & 88.15 & 11103 \\
\hline 442 & 99.97 & 4393 & 40.43 & 11718 \\
\hline 4692 & 41.44 & 4630 & 41.01 & 12376 \\
\hline $49 \times 6$ & 43.74 & 4910 & 4423 & 13098 \\
\hline 5301 & 45.76 & 5241 & 46.28 & 13081 \\
\hline
\end{tabular}




\begin{tabular}{|c|c|c|c|c|}
\hline Po $(m)$ & $\operatorname{Nu}(\mathrm{m})$ & Ro 0 & Nu $(0)$ & $\mathrm{R}_{\mathrm{m}}$ \\
\hline 5004 & 47.92 & 5062 & 48.48 & 14756 \\
\hline 8017 & 60.64 & 6850 & 8121 & 15808 \\
\hline 2451 & 54.12 & 0370 & 54.74 & 17017 \\
\hline coest & 67.58 & 0843 & 88.21 & 18255 \\
\hline 7435 & 0.27 & 7352 & 00.08 & 19611 \\
\hline 8081 & es.74 & 7971 & 94.46 & 21233 \\
\hline 8777 & 67.80 & 8870 & 68.65 & 203152 \\
\hline 9101 & 70.63 & 0009 & 71.43 & 24244 \\
\hline 8469 & 72.10 & 0563 & 7201 & 24977 \\
\hline 9780 & 74.17 & 2881 & 75.01 & 25024 \\
\hline 45666 & 229.77 & 45155 & 232.37 & 120454 \\
\hline 48504 & 239.06 & 47962 & 241.77 & 127940 \\
\hline 51300 & 251.03 & 50726 & 253.87 & 135314 \\
\hline 53988 & 202.48 & 53384 & 265.45 & 142404 \\
\hline 57710 & 276.80 & 57065 & 279.93 & 150023 \\
\hline 62085 & 292.51 & 61390 & 205.82 & 163761 \\
\hline 67123 & 308.69 & 66372 & 312.18 & 177050 \\
\hline 71802 & 324.83 & 70999 & 328.51 & 189393 \\
\hline 75618 & 340.85 & 74772 & 344.79 & 189458 \\
\hline 79130 & 350.40 & 78245 & 354.36 & 208721 \\
\hline
\end{tabular}

Heat transfer $(C 80$, data set $(8)): p / e=80, \theta / D=0.04, d_{m}=49.82 \mathrm{~mm}, \nabla_{R}(m)=1.306, \nabla_{R}(D)=1.312$ semicircular, flattened (radius $=18 \mathrm{~mm}$ )

\begin{tabular}{|r|r|r|r|r|}
\hline$R_{0}(m)$ & $N u(m)$ & $R_{9}$ & $N_{u_{1}}$ & $R_{\omega_{m}}$ \\
\hline 2002 & 12.00 & 1892 & 12.05 & 2614 \\
\hline 2120 & 12.20 & 2110 & 12.26 & 2769 \\
\hline 2247 & 12.57 & 2236 & 12.63 & 2935 \\
\hline 2371 & 12.71 & 2360 & 12.77 & 3097 \\
\hline 2506 & 13.11 & 2494 & 13.17 & 3273 \\
\hline 2021 & 13.48 & 2600 & 13.55 & 3423 \\
\hline 2723 & 13.7 & 2710 & 13.84 & 355 \\
\hline 2874 & 14.15 & 2860 & 14.22 & 3754 \\
\hline 3035 & 14.61 & 3021 & 14.68 & 3964 \\
\hline
\end{tabular}




\begin{tabular}{|c|c|c|c|c|}
\hline Po $(m)$ & $\operatorname{Nu}(\mathrm{m})$ & $R_{1}$ & $\mathrm{Nu}_{1}$ & $\boldsymbol{P o s}_{\mathrm{m}}$ \\
\hline$\$ 176$ & 14.97 & s161 & 15.04 & 4148 \\
\hline 2010 & 15.42 & $3 \times 24$ & 16.50 & 4503 \\
\hline saps & 15.89 & 3479 & 16.07 & 4585 \\
\hline 8902 & 16.30 & 3014 & 16.47 & 474 \\
\hline 3787 & 16.82 & . 3760 & 17.00 & 4046 \\
\hline $\sec 2$ & 17.38 & 3964 & 17.47 & 5071 \\
\hline 4000 & 17.78 & 8000 & 17.87 & 5224 \\
\hline 4115 & 1822 & 4005 & 18.30 & 5374 \\
\hline 4188 & 18.46 & 4148 & 18.55 & 5413 \\
\hline 4552 & 19.16 & 4331 & 10.25 & 5684 \\
\hline 4522 & 20.06 & 4500 & 20.15 & 5006 \\
\hline 4705 & 20.82 & 4683 & 20.82 & 6145 \\
\hline 4082 & 21.91 & 4938 & 22.02 & 6481 \\
\hline 5120 & 22.64 & 5095 & 22.75 & 6687 \\
\hline 5009 & 23.67 & 5373 & 23.79 & 7052 \\
\hline 5642 & 24.61 & 8815 & 24.73 & 7350 \\
\hline 5879 & 25.55 & 5851 & 25.68 & 7678 \\
\hline 6135 & 28.50 & 6105 & 28.71 & 8013 \\
\hline 6484 & 27.82 & 6452 & 28.05 & 8468 \\
\hline 6775 & 28.80 & 6743 & 29.12 & 8849 \\
\hline 7171 & 50.30 & 7136 & 30.54 & 9066 \\
\hline 7445 & 31.44 & 7410 & 31.60 & ires \\
\hline 7818 & 82.62 & 7781 & 32.78 & 10212 \\
\hline E211 & 35.87 & 8172 & 34.13 & 10725 \\
\hline 6502 & 35.02 & 8521 & 35.19 & 11188 \\
\hline 8015 & 83.10 & 8872 & 36.27 & 11644 \\
\hline 9165 & 56.60 & 0121 & 36.97 & 11071 \\
\hline 2483 & 88.01 & 9437 & 38.20 & 12088 \\
\hline 8374 & 80.50 & 8827 & 39.60 & 12097 \\
\hline 10258 & 40.98 & 10189 & 41.17 & 13372 \\
\hline 10680 & 42.33 & 10000 & 42.54 & 13024 \\
\hline 11108 & $\$ 4.14$ & 11055 & 44.35 & 14508 \\
\hline 11715 & 45.76 & 11659 & 45.98 & 15301 \\
\hline
\end{tabular}




\begin{tabular}{|c|c|c|c|c|}
\hline$A_{0}(m)$ & Nu (m) & Ro 0 & Nu 0 & $\boldsymbol{n}_{m}$ \\
\hline 12000 & 47.86 & 12331 & 48.18 & 16183 \\
\hline 13113 & 50.25 & 18051 & 80.49 & 17128 \\
\hline 13850 & 82.65 & 13764 & 8280 & 18064 \\
\hline 14580 & 54.55 & 14311 & $\mathbf{5 4 . 8 1}$ & 18782 \\
\hline 15200 & 56.75 & 15136 & 67.03 & 18065 \\
\hline 15650 & 88.82 & 15783 & 50.21 & 20714 \\
\hline 16773 & 61.18 & 180003 & 61.47 & 21808 \\
\hline 1767 & 68.43 & 17502 & $e 3.73$ & 20088 \\
\hline 18643 & 68.46 & 18554 & 66.78 & 24350 \\
\hline 10788 & 60.54 & 18093 & 69.88 & 25845 \\
\hline 20028 & 72.82 & 20828 & 73.17 & 27335 \\
\hline 22197 & 75.55 & 22001 & 75.91 & 20992 \\
\hline 23344 & 79.50 & 23232 & 79.88 & 30490 \\
\hline 24497 & 82.01 & 24380 & 82.41 & 31997 \\
\hline 26075 & 85.63 & 25050 & 66.05 & 34057 \\
\hline 28012 & 80.43 & 27877 & $\infty 0.87$ & 36587 \\
\hline 29459 & 84.42 & 20317 & 94.88 & 38477 \\
\hline 30090 & 97.86 & 30832 & $\bullet 8.44$ & 40464 \\
\hline 32650 & 102.22 & 32493 & 102.71 & 42645 \\
\hline 34852 & 106.85 & 34685 & 107.47 & 45521 \\
\hline 36834 & 112.15 & 36558 & 112.69 & 48110 \\
\hline 38700 & 115.66 & 38524 & 116.42 & 50559 \\
\hline 40382 & 120.04 & 40190 & 120.62 & 52758 \\
\hline 43300 & 125.34 & 43003 & 125.84 & 56556 \\
\hline 45375 & 130.41 & 45158 & 131.03 & 59266 \\
\hline 47440 & 134.53 & 47202 & 135.18 & 61975 \\
\hline 49820 & 138.70 & 49590 & 139.46 & 65084 \\
\hline 52627 & 143.40 & 52374 & 144.18 & 68737 \\
\hline 54837 & 147.82 & 54574 & 148.53 & 71624 \\
\hline 57221 & 152.00 & 50047 & 153.34 & 74738 \\
\hline 600262 & 157.80 & 59973 & 158.75 & 78710 \\
\hline 62749 & 162.80 & 62448 & 163.7 & 81858 \\
\hline 65850 & 168.15 & 65534 & 168.96 & 86008 \\
\hline
\end{tabular}




\begin{tabular}{|c|c|c|c|c|}
\hline$D_{0}(m)$ & $N u(m)$ & Ro (i) & Nu $(1)$ & $\mathbf{R o}_{m}$ \\
\hline 00275 & 173.22 & 67948 & 174.06 & 60178 \\
\hline 70790 & 178.07 & 70451 & 178.83 & 02461 \\
\hline T2023 & 181.12 & 72033 & 182.00 & ese25 \\
\hline 75000 & 184.36 & 74670 & 185.25 & 97999 \\
\hline
\end{tabular}

Nunner (1956): Enhancement factors

\begin{tabular}{|c|c|c|c|c|c|}
\hline$e / D$ & $\mathrm{p} / \mathrm{e}$ (set) & $\nabla_{A}^{\circ}$ & $i^{*}$ & $0_{i}^{+}$ & $\Phi_{h_{\text {max }}}$ \\
\hline 0.08 & $10(1)$ & 1.518 & 0.43 & 7.48 & 28 \\
\hline 0.08 & 5 (2) & 1.246 & 0.5 & 8.48 & 2.6 \\
\hline 0.08 & $20(3)$ & 2.456 & 0.245 & 3.06 & 2.5 \\
\hline 0.04 & $20(4)$ & 1.262 & 0.631 & 5.48 & 2.2 \\
\hline 0.04 & $20(5)$ & 1.262 & 0.631 & 4.15 & 2.1 \\
\hline 0.08 & $20(6)$ & 1.005 & 0.724 & 4.24 & 2.4 \\
\hline 0.04 & $80(7)$ & 0.989 & 0.866 & 2.31 & 1.4 \\
\hline \multicolumn{2}{|c|}{ smooth } & 1 & 1 & 1 & 1 \\
\hline
\end{tabular}

+ Based on $R e_{m}=50,000$

- Based on the data of Table 1 
Table A.9

Nikuradse (1933)

Friction data for sand-grain roughened tubes

$Q / D=0.033, \downarrow_{R}=0.921, \downarrow_{f}=1.031$

\begin{tabular}{|c|c|c|c|c|c|}
\hline $\log (R \theta)$ & $\log (4000)$ & $\mathbf{R e}_{\boldsymbol{\theta}}$ & 1 & $\boldsymbol{R} \boldsymbol{\theta}_{\mathrm{m}}$ & $f_{m}$ \\
\hline 2.739 & 1.056 & 549 & 0.02841 & 505 & 0.02930 \\
\hline 2.884 & 0.911 & 766 & 0.02037 & 705 & 0.02101 \\
\hline 3.016 & 0.793 & 1037 & 0.01554 & 955 & 0.01602 \\
\hline 3.109 & 0.698 & 1285 & 0.01246 & 1184 & 0.01285 \\
\hline 3.184 & 0.620 & 1528 & 0.01043 & 1407 & 0.01076 \\
\hline 3.345 & 0.503 & 2211 & 0.00796 & 2036 & 0.00821 \\
\hline 3.358 & 0.492 & 2281 & 0.00776 & 2100 & 0.00800 \\
\hline 3.388 & 0.493 & 2443 & 0.00777 & 2249 & 0.00801 \\
\hline 3.386 & 0.502 & 2430 & 0.00794 & 2237 & 0.00819 \\
\hline 3.429 & 0.522 & 2688 & 0.00832 & 2475 & 0.00858 \\
\hline 3.458 & 0.538 & 2870 & 0.00862 & 2643 & 0.00889 \\
\hline 3.480 & 0.543 & 3022 & 0.00873 & 2782 & 0.00901 \\
\hline 3.454 & 0.555 & 2843 & 0.00898 & 2617 & 0.00926 \\
\hline 3.475 & 0.576 & 2986 & 0.00942 & 2750 & 0.00971 \\
\hline 3.513 & 0.574 & 3261 & 0.00937 & 3003 & 0.00966 \\
\hline 3.534 & 0.588 & 3423 & 0.00968 & 3151 & 0.00999 \\
\hline 3.529 & 0.604 & 3379 & 0.01004 & 3111 & 0.01035 \\
\hline 3.558 & 0.603 & 3612 & 0.01003 & 3325 & 0.01034 \\
\hline 3.564 & 0.620 & 3666 & 0.01042 & 3376 & 0.01074 \\
\hline 3.589 & 0.625 & 3881 & 0.01054 & 3573 & 0.01087 \\
\hline 3.602 & 0.636 & 4004 & 0.01081 & 3686 & 0.01114 \\
\hline 3.633 & 0.641 & 4293 & 0.01095 & 3953 & 0.01129 \\
\hline 3.643 & 0.652 & 4396 & 0.01121 & 4047 & 0.01156 \\
\hline 3.666 & 0.651 & 4638 & 0.01120 & 4271 & 0.01155 \\
\hline 3.687 & 0.655 & 4863 & 0.01130 & 4477 & 0.01166 \\
\hline
\end{tabular}




\begin{tabular}{|c|c|c|c|c|c|}
\hline $\log (R \theta)$ & $\log (400)$ & Re & 1 & $\mathbf{R} \boldsymbol{e}_{\mathrm{m}}$ & $t_{m}$ \\
\hline 3.695 & 0.661 & 4958 & 0.01146 & 4565 & 0.01182 \\
\hline 3.713 & 0.670 & 5164 & 0.01170 & 4755 & 0.01206 \\
\hline 3.743 & 0.669 & 5538 & 0.01168 & 5098 & 0.01204 \\
\hline 3.765 & 0.679 & 5818 & 0.01194 & 5357 & 0.01231 \\
\hline 3.768 & 0.686 & 5862 & 0.01214 & 5397 & 0.01252 \\
\hline 3.820 & 0.692 & 6611 & 0.01229 & 6087 & 0.01267 \\
\hline 3.859 & 0.699 & 7227 & 0.01250 & 6654 & 0.01289 \\
\hline 3.908 & 0.705 & 8090 & 0.01268 & 7448 & 0.01307 \\
\hline 3.964 & 0.709 & 9212 & 0.01279 & 8482 & 0.01319 \\
\hline 4.006 & 0.723 & 10136 & 0.01320 & 9333 & 0.01362 \\
\hline 4.046 & 0.728 & 11105 & 0.01336 & 10225 & 0.01378 \\
\hline 4.074 & 0.728 & 11870 & 0.01336 & 10929 & 0.01378 \\
\hline 4.081 & 0.736 & 12050 & 0.01362 & 11094 & 0.01405 \\
\hline 4.119 & 0.744 & 13145 & 0.01386 & 12102 & 0.01429 \\
\hline 4.137 & 0.734 & 13707 & 0.01354 & 12620 & 0.01397 \\
\hline 4.181 & 0.739 & 15179 & 0.01371 & 13975 & 0.01414 \\
\hline 4.208 & 0.739 & 16137 & 0.01371 & 14857 & 0.01414 \\
\hline 4.197 & 0.748 & 15743 & 0.01398 & 14495 & 0.01442 \\
\hline 4.219 & 0.753 & 16576 & 0.01415 & 15262 & 0.01459 \\
\hline 4.267 & 0.753 & 18495 & 0.01416 & 17028 & 0.01460 \\
\hline 4.294 & 0.748 & 19683 & 0.01398 & 18123 & 0.01442 \\
\hline 4.313 & 0.761 & 20547 & 0.01443 & 18918 & 0.01488 \\
\hline 4.346 & 0.756 & 22199 & 0.01424 & 20439 & 0.01468 \\
\hline 4.372 & 0.770 & 23524 & 0.01472 & 21659 & 0.01518 \\
\hline 4.397 & 0.765 & 24929 & 0.01456 & 22952 & 0.01501 \\
\hline 4.419 & 0.767 & 26219 & 0.01461 & 24140 & 0.01506 \\
\hline 4.435 & 0.776 & 27253 & 0.01491 & 25092 & 0.01538 \\
\hline 4.450 & 0.768 & 28175 & 0.01466 & 25941 & 0.01512 \\
\hline 4.476 & 0.778 & 29921 & 0.01501 & 27549 & 0.01548 \\
\hline
\end{tabular}




\begin{tabular}{|c|c|c|c|c|c|}
\hline $\log (R \theta)$ & $\log (4001)$ & $\mathbf{R e}_{\boldsymbol{\theta}}$ & $t$ & $\mathbf{R}_{\boldsymbol{\theta}_{\mathrm{m}}}$ & $f_{m}$ \\
\hline 4.481 & 0.772 & 30277 & 0.01478 & 27876 & 0.01524 \\
\hline 4.508 & 0.771 & 32223 & 0.01475 & 29668 & 0.01521 \\
\hline 4.534 & 0.773 & 34220 & 0.01482 & 31506 & 0.01528 \\
\hline 4.559 & 0.773 & 36224 & 0.01483 & 33352 & 0.01529 \\
\hline 4.594 & 0.776 & 39262 & 0.01493 & 36149 & 0.01540 \\
\hline 4.630 & 0.773 & 42692 & 0.01483 & 39307 & 0.01529 \\
\hline 4.686 & 0.779 & 48512 & 0.01501 & 44665 & 0.01548 \\
\hline 4.723 & 0.771 & 52864 & 0.01475 & 48672 & 0.01521 \\
\hline 4.730 & 0.777 & 53722 & 0.01498 & 49462 & 0.01544 \\
\hline 4.812 & 0.777 & 64898 & 0.01494 & 59752 & 0.01541 \\
\hline 4.838 & 0.769 & 68847 & 0.01470 & 63388 & 0.01516 \\
\hline 4.858 & 0.776 & 72100 & 0.01494 & 66383 & 0.01541 \\
\hline 4.882 & 0.772 & 76241 & 0.01480 & 70196 & 0.01526 \\
\hline 4.893 & 0.780 & 78231 & 0.01505 & 72028 & 0.01552 \\
\hline 4.913 & 0.772 & 81841 & 0.01479 & 75351 & 0.01525 \\
\hline 4.933 & 0.778 & 85616 & 0.01498 & 78827 & 0.01545 \\
\hline 4.957 & 0.777 & 90630 & 0.01496 & 83444 & 0.01542 \\
\hline 4.990 & 0.776 & 97706 & 0.01493 & 89958 & 0.01540 \\
\hline 5.004 & 0.783 & 101013 & 0.01517 & 93003 & 0.01564 \\
\hline 5.063 & 0.781 & 115524 & 0.01508 & 106364 & 0.01555 \\
\hline 5.088 & 0.775 & 122421 & 0.01489 & 112714 & 0.01536 \\
\hline 5.106 & 0.782 & 127657 & 0.01512 & 117534 & 0.01559 \\
\hline 5.133 & 0.783 & 135715 & 0.01515 & 124954 & 0.01563 \\
\hline 5.148 & 0.774 & 140460 & 0.01486 & 129322 & 0.01533 \\
\hline 5.156 & 0.785 & 143355 & 0.01524 & 131988 & 0.01572 \\
\hline 5.178 & 0.781 & 150614 & 0.01510 & 138671 & 0.01557 \\
\hline 5.209 & 0.776 & 161676 & 0.01491 & 148856 & 0.01538 \\
\hline 5.238 & 0.775 & 172992 & 0.01488 & 159275 & 0.01535 \\
\hline 5.232 & 0.783 & 170411 & 0.01516 & 156898 & 0.01563 \\
\hline
\end{tabular}




\begin{tabular}{|c|c|c|c|c|c|}
\hline $\log (R \theta)$ & $\log (4001)$ & $R \theta$ & $f$ & $R \theta_{m}$ & $f_{m}$ \\
\hline 5.305 & 0.777 & 201705 & 0.01497 & 185711 & 0.01544 \\
\hline 5.348 & 0.773 & 222650 & 0.01483 & 204995 & 0.01530 \\
\hline 5.376 & 0.783 & 237723 & 0.01518 & 218873 & 0.01565 \\
\hline 5.403 & 0.780 & 252729 & 0.01506 & 232689 & 0.01553 \\
\hline 5.437 & 0.780 & 273631 & 0.01507 & 251934 & 0.01554 \\
\hline 5.456 & 0.776 & 285948 & 0.01492 & 263274 & 0.01539 \\
\hline 5.511 & 0.782 & 324229 & 0.01513 & 298519 & 0.01561 \\
\hline 5.565 & 0.777 & 367635 & 0.01498 & 338484 & 0.01544 \\
\hline 5.586 & 0.783 & 385837 & 0.01516 & 355242 & 0.01564 \\
\hline 5.614 & 0.780 & 411515 & 0.01508 & 378884 & 0.01555 \\
\hline 5.690 & 0.784 & 490234 & 0.01520 & 451361 & 0.01568 \\
\hline 5.792 & 0.782 & 618878 & 0.01513 & 569804 & 0.01560 \\
\hline 5.828 & 0.783 & 672227 & 0.01518 & 618923 & 0.01565 \\
\hline 5.862 & 0.778 & 728607 & 0.01499 & 670832 & 0.01546 \\
\hline 5.883 & 0.778 & 763859 & 0.01501 & 703289 & 0.01548 \\
\hline 5.926 & 0.780 & 843178 & 0.01507 & 776318 & 0.01554 \\
\hline 5.957 & 0.779 & 905106 & 0.01503 & 833336 & 0.01550 \\
\hline 5.995 & 0.780 & 988420 & 0.01506 & 910044 & 0.01553 \\
\hline 6.020 & 0.783 & 1046330 & 0.01516 & 963350 & 0.01564 \\
\hline
\end{tabular}

$e / D=0.016, \downarrow_{R}=0.890, \downarrow_{1}=1.055$

\begin{tabular}{|r|r|r|r|r|r|}
\hline $\log (R e)$ & $\log (400)$ & $R e$ & $f$ & $R e_{m}$ & $f_{m}$ \\
\hline 2815 & 0.976 & 653 & 0.02366 & 581 & 0.02495 \\
\hline 2900 & 0.903 & 795 & 0.02002 & 707 & 0.02111 \\
\hline 3.065 & 0.735 & 1162 & 0.01358 & 1034 & 0.01433 \\
\hline 3.226 & 0.584 & 1681 & 0.00959 & 1496 & 0.01011 \\
\hline 3.310 & 0.528 & 2042 & 0.00844 & 1817 & 0.00890 \\
\hline 3.373 & 0.482 & 2361 & 0.00758 & 2100 & 0.00800 \\
\hline
\end{tabular}




\begin{tabular}{|c|c|c|c|c|c|}
\hline $\log (R \theta)$ & $\log (400)$ & Re & I & $R \theta_{m}$ & $f_{m}$ \\
\hline 3.415 & 0.497 & 2603 & 0.00786 & 2316 & 0.00829 \\
\hline 3.555 & 0.561 & 3592 & 0.00909 & 3196 & 0.00959 \\
\hline 3.597 & 0.577 & 3953 & 0.00944 & 3516 & 0.00995 \\
\hline 3.640 & 0.580 & 4363 & 0.00950 & 3881 & 0.01002 \\
\hline 3.664 & 0.593 & 4614 & 0.00980 & 4104 & 0.01033 \\
\hline 3.717 & 0.586 & 5214 & 0.00964 & 4639 & 0.01017 \\
\hline 3.766 & 0.592 & 5830 & 0.00978 & 5187 & 0.01031 \\
\hline 3.760 & 0.585 & 5750 & 0.00962 & 5115 & 0.01014 \\
\hline 3.795 & 0.574 & 6232 & 0.00937 & 5544 & 0.00988 \\
\hline 3.834 & 0.578 & 6820 & 0.00945 & 6067 & 0.00997 \\
\hline 3.882 & 0.577 & 7618 & 0.00944 & 6777 & 0.00995 \\
\hline 3.889 & 0.570 & 7742 & 0.00928 & 6887 & 0.00979 \\
\hline 3.942 & 0.578 & 8750 & 0.00947 & 7784 & 0.00999 \\
\hline 3.941 & 0.569 & 8722 & 0.00927 & 7759 & 0.00978 \\
\hline 3.989 & 0.572 & 9742 & 0.00933 & 8666 & 0.00984 \\
\hline 4.017 & 0.580 & 10390 & 0.00951 & 9243 & 0.01003 \\
\hline 4.057 & 0.577 & 11395 & 0.00944 & 10137 & 0.00996 \\
\hline 4.084 & 0.587 & 12127 & 0.00965 & 10789 & 0.01018 \\
\hline 4.137 & 0.582 & 13707 & 0.00956 & 12194 & 0.01008 \\
\hline 4.136 & 0.590 & 13692 & 0.00973 & 12181 & 0.01026 \\
\hline 4.196 & 0.610 & 15693 & 0.01018 & 13960 & 0.01073 \\
\hline 4.198 & 0.594 & 15760 & 0.00982 & 14020 & 0.01036 \\
\hline 4.267 & 0.605 & 18475 & 0.01006 & 16435 & 0.01061 \\
\hline 4.296 & 0.615 & 19747 & 0.01031 & 17567 & 0.01087 \\
\hline 4.311 & 0.607 & 20459 & 0.01011 & 18201 & 0.01067 \\
\hline 4.337 & 0.624 & 21751 & 0.01052 & 19349 & 0.01109 \\
\hline 4.395 & 0.628 & 24822 & 0.01060 & 22082 & 0.01119 \\
\hline 4.427 & 0.635 & 26731 & 0.01078 & 23780 & 0.01137 \\
\hline 4.451 & 0.627 & 28266 & 0.01058 & 25146 & 0.01116 \\
\hline
\end{tabular}




\begin{tabular}{|c|c|c|c|c|c|}
\hline $\log \left(R_{\theta}\right)$ & $\log (4001)$ & Re & $f$ & $\mathbf{R} \boldsymbol{\theta}_{\mathrm{m}}$ & $f_{m}$ \\
\hline 4.504 & 0.642 & 31879 & 0.01097 & 28359 & 0.01157 \\
\hline 4.566 & 0.634 & 36773 & 0.01077 & 32713 & 0.01136 \\
\hline 4.612 & 0.640 & 40897 & 0.01092 & 36382 & 0.01152 \\
\hline 4.635 & 0.648 & 43153 & 0.01113 & 38389 & 0.01173 \\
\hline 4.672 & 0.645 & 47024 & 0.01104 & 41833 & 0.01165 \\
\hline 4.739 & 0.652 & 54829 & 0.01122 & 48777 & 0.01184 \\
\hline 4.797 & 0.646 & 62639 & 0.01107 & 55724 & 0.01168 \\
\hline 4.829 & 0.658 & 67384 & 0.01136 & 59945 & 0.01199 \\
\hline 4.856 & 0.658 & 71791 & 0.01137 & 63866 & 0.01199 \\
\hline 4.901 & 0.655 & 79587 & 0.01129 & 70801 & 0.01191 \\
\hline 4.933 & 0.656 & 85800 & 0.01132 & 76328 & 0.01194 \\
\hline 4.962 & 0.661 & 91609 & 0.01145 & 81496 & 0.01208 \\
\hline 4.990 & 0.665 & 97706 & 0.01155 & 86919 & 0.01218 \\
\hline 5.034 & 0.656 & 108199 & 0.01133 & 96254 & 0.01195 \\
\hline 5.063 & 0.656 & 115648 & 0.01133 & 102881 & 0.01195 \\
\hline 5.069 & 0.666 & 117274 & 0.01158 & 104327 & 0.01221 \\
\hline 5.103 & 0.663 & 126837 & 0.01150 & 112835 & 0.01213 \\
\hline 5.134 & 0.660 & 136153 & 0.01144 & 121122 & 0.01206 \\
\hline 5.169 & 0.665 & 147414 & 0.01157 & 131140 & 0.01220 \\
\hline 5.167 & 0.656 & 146940 & 0.01133 & 130718 & 0.01195 \\
\hline 5.180 & 0.645 & 151262 & 0.01105 & 134563 & 0.01166 \\
\hline 5.238 & 0.665 & 172807 & 0.01156 & 153730 & 0.01219 \\
\hline 5.228 & 0.656 & 168953 & 0.01132 & 150301 & 0.01194 \\
\hline 5.259 & 0.652 & 181362 & 0.01123 & 161340 & 0.01184 \\
\hline 5.268 & 0.665 & 185300 & 0.01157 & 164844 & 0.01220 \\
\hline 5.314 & 0.663 & 205863 & 0.01151 & 183137 & 0.01214 \\
\hline 5.317 & 0.653 & 207639 & 0.01126 & 184717 & 0.01187 \\
\hline 5.344 & 0.655 & 220983 & 0.01130 & 196587 & 0.01192 \\
\hline 5.341 & 0.663 & 219093 & 0.01151 & 194906 & 0.01214 \\
\hline
\end{tabular}




\begin{tabular}{|c|c|c|c|c|c|}
\hline $\log (R \theta)$ & $\log (400)$ & $R \theta$ & $f$ & $R \theta_{m}$ & $f_{m}$ \\
\hline 5.396 & 0.662 & 248690 & 0.01147 & 221236 & 0.01210 \\
\hline 5.424 & 0.665 & 265241 & 0.01157 & 235960 & 0.01220 \\
\hline 5.452 & 0.661 & 282894 & 0.01145 & 251664 & 0.01207 \\
\hline 5.475 & 0.661 & 298819 & 0.01144 & 265831 & 0.01207 \\
\hline 5.519 & 0.667 & 330557 & 0.01162 & 294065 & 0.01226 \\
\hline 5.543 & 0.661 & 349166 & 0.01146 & 310620 & 0.01209 \\
\hline 5.599 & 0.665 & 396761 & 0.01156 & 352960 & 0.01219 \\
\hline 5.662 & 0.662 & 459151 & 0.01148 & 408463 & 0.01211 \\
\hline 5.723 & 0.662 & 527938 & 0.01148 & 469656 & 0.01211 \\
\hline 5.811 & 0.666 & 646735 & 0.01159 & 575338 & 0.01222 \\
\hline 5.865 & 0.668 & 732529 & 0.01163 & 651661 & 0.01226 \\
\hline 5.915 & 0.665 & 822608 & 0.01155 & 731796 & 0.01218 \\
\hline 5.964 & 0.665 & 819804 & 0.01156 & 818262 & 0.01219 \\
\hline 6.012 & 0.669 & 1028480 & 0.01166 & 914943 & 0.01230 \\
\hline & & & & & \\
\hline
\end{tabular}


Table A.10

Blackwelder and Kreith (1970)

Heat transfer data for circular tube with twisted tape inserts

Smooth tube data

\begin{tabular}{|l|r|r|}
\hline Re & Nu & \\
\hline 2994 & 7.04 \\
\hline 3751 & 7.36 \\
\hline 3334 & 9.14 \\
\hline 6103 & 16.27 \\
\hline 6275 & 15.70 \\
\hline 6575 & 16.70 \\
\hline 11487 & 26.65 \\
\hline 19586 & 41.01 \\
\hline 24491 & 45.02 \\
\hline 43233 & 65.56 \\
\hline 47391 & 77.71 \\
\hline 53132 & 91.54 \\
\hline 53967 & 88.01 \\
\hline 59672 & 87.58 \\
\hline 65524 & 95.69 \\
\hline & & \\
\hline & & \\
\hline
\end{tabular}


$\mathrm{P} / \mathrm{D}=3.54, \nabla_{A}=2.45$

\begin{tabular}{|c|c|c|c|}
\hline$R_{e}$ & $\mathrm{Nu}$ & $\boldsymbol{R} e_{m}$ & $\mathrm{Nu}_{\mathrm{m}}$ \\
\hline 1035 & 5.36 & 2536 & 5.36 \\
\hline 1102 & 5.57 & 2699 & 5.57 \\
\hline 1547 & 10.54 & 3790 & 10.54 \\
\hline 2035 & 13.28 & 4983 & 13.28 \\
\hline 1928 & 13.68 & 4723 & 13.68 \\
\hline 2017 & 14.51 & 4940 & 14.51 \\
\hline 1932 & 15.24 & 4731 & 15.24 \\
\hline 2662 & 18.37 & 6518 & 18.37 \\
\hline 2722 & 21.96 & 6667 & 21.96 \\
\hline 2989 & 22.22 & 7321 & 22.22 \\
\hline 3392 & 27.22 & 8308 & 27.22 \\
\hline 3579 & 27.36 & 8766 & 27.36 \\
\hline 4898 & 33.91 & 11995 & 33.91 \\
\hline 4906 & 32.28 & 12016 & 32.28 \\
\hline 11000 & 55.17 & 26940 & 55.17 \\
\hline 19928 & 89.32 & 48808 & 89.32 \\
\hline 20992 & 91.24 & 51412 & 91.24 \\
\hline 20992 & 96.95 & 51412 & 96.95 \\
\hline 47556 & 149.68 & 116470 & 149.68 \\
\hline 47391 & 157.22 & 116067 & 157.22 \\
\hline 67366 & 19265 & 164990 & 192.65 \\
\hline 66324 & 184.01 & 162437 & 184.01 \\
\hline
\end{tabular}




$$
P / D=8.5, \downarrow_{A}=2.32
$$

\begin{tabular}{|c|c|c|c|}
\hline Re & $\mathrm{Nu}$ & $\boldsymbol{R} \boldsymbol{e}_{\mathrm{m}}$ & $\mathbf{N u}$ \\
\hline 1127 & 5.35 & 2611 & 5.35 \\
\hline 1451 & 7.23 & 3363 & 7.23 \\
\hline 1434 & 7.76 & 3322 & 7.76 \\
\hline 1824 & 8.43 & 4227 & 9.43 \\
\hline 2238 & 11.53 & 5186 & 11.53 \\
\hline 2760 & 15.54 & 6395 & 15.54 \\
\hline 3288 & 20.81 & 7618 & 20.81 \\
\hline 3586 & 19.39 & 8308 & 19.39 \\
\hline 4293 & 21.36 & 9948 & 21.36 \\
\hline 5936 & 29.84 & 13755 & 29.84 \\
\hline 7170 & 30.58 & 16614 & 30.58 \\
\hline 14844 & 47.29 & 34395 & 47.29 \\
\hline 21396 & 66.94 & 49575 & 66.94 \\
\hline 21845 & 72.77 & 50616 & 72.77 \\
\hline 31432 & 96.31 & 72829 & 96.31 \\
\hline 47638 & 118.80 & 110380 & 118.80 \\
\hline 49575 & 138.13 & 114867 & 138.13 \\
\hline 65072 & 155.43 & 150775 & 155.43 \\
\hline 70470 & 168.15 & 163283 & 168.15 \\
\hline
\end{tabular}




$$
P / D=22.0, \nabla_{R}=2.18
$$

\begin{tabular}{|c|c|c|c|}
\hline Re & $\mathbf{N u}$ & $\mathbf{R} \boldsymbol{\theta}_{\mathrm{m}}$ & $\mathrm{Nu}_{m}$ \\
\hline 1202 & 4.85 & 2616 & 4.85 \\
\hline 1843 & 7.35 & 4013 & 7.35 \\
\hline 1847 & 7.82 & 4020 & 7.82 \\
\hline 2571 & 10.22 & 5597 & 10.22 \\
\hline 2699 & 10.96 & 5875 & 10.96 \\
\hline 3985 & 13.41 & 8676 & 13.41 \\
\hline 5645 & 20.14 & 12290 & 20.14 \\
\hline 5895 & 19.45 & 12834 & 19.45 \\
\hline 6199 & 20.14 & 13495 & 20.14 \\
\hline 11871 & 35.50 & 25843 & 35.50 \\
\hline 20524 & 55.17 & 44680 & 55.17 \\
\hline 21211 & 60.47 & 46175 & 60.47 \\
\hline 28974 & 63.10 & 63074 & 63.10 \\
\hline 34335 & 76.07 & 74746 & 76.07 \\
\hline 43158 & 89.47 & 93953 & 89.47 \\
\hline 45225 & $\mathbf{9 3 . 5 1}$ & 98453 & 93.51 \\
\hline 46416 & 90.06 & 101045 & 90.06 \\
\hline 73082 & 121.75 & 159094 & 121.75 \\
\hline 71578 & 129.36 & 155821 & 129.36 \\
\hline
\end{tabular}


Table A.11

Knudsen and Katz (1950)

Heat transfer data for smooth and rough annuli

(a) smooth tube: $\downarrow_{R}=1.692$

\begin{tabular}{|c|c|c|c|c|c|c|c|}
\hline Re & Nuppes & $\mathbf{R}_{m}$ & $N u_{m} P^{O P .4}$ & $\mathbf{R}$ & NuPp ${ }^{0.4}$ & $\boldsymbol{R}_{\mathrm{m}}$ & $\mathrm{Nu}_{m} \mathbf{P r}^{\mathrm{O} .4}$ \\
\hline 2615 & 33.27 & 405 & 30.27 & 20281 & e8.14 & 39398 & $\$ 8.14$ \\
\hline 3224 & 34.46 & 6455 & 34.46 & 23740 & 101.11 & 40175 & 101.11 \\
\hline 3763 & 25.27 & $\infty$ & 35.27 & 20653 & 128.83 & 50182 & 126.83 \\
\hline 5264 & 37.63 & 000 & $\$ 7.63$ & 31034 & 128.67 & 52520 & 126.67 \\
\hline 7940 & 40.84 & 13437 & 10.94 & 57370 & 147.21 & 65256 & 147.21 \\
\hline 8739 & 50.30 & 16481 & 50.30 & 42904 & 170.42 & 72608 & 170.42 \\
\hline 11310 & 55.57 & 10141 & 55.57 & 45847 & 169.75 & 77587 & 169.75 \\
\hline 12898 & 60.14 & 21828 & 60.14 & 45907 & 183.47 & 77688 & 183.47 \\
\hline 15205 & 69.80 & 25883 & 69.80 & 49119 & 183.47 & 83125 & 183.47 \\
\hline 17647 & 84.45 & 20864 & 84.45 & 75550 & 254.96 & 127854 & 254.96 \\
\hline
\end{tabular}

(b) Transverse-fin tubes

Tube 1: $\psi_{R}=1.99$

\begin{tabular}{|c|c|c|c|c|c|c|c|}
\hline Ro & Nurpe.4 & $\mathbf{R o}_{\mathrm{m}}$ & $\mathrm{Nu}_{m} \mathrm{Pr}^{\mathrm{O} \cdot 4}$ & Ro & Nupo.4 & $\mathbf{R}_{\boldsymbol{m}}$ & $\mathrm{Nu}_{\mathrm{m}} \mathrm{Pr}^{0.4}$ \\
\hline 1276 & 21.00 & 2539 & 21.00 & 17841 & 117.05 & 35501 & 117.05 \\
\hline 1706 & 22.37 & 3304 & 22.37 & 21613 & 144.77 & 43006 & 144.77 \\
\hline 2141 & 21.58 & 4260 & 21.58 & 27386 & 168.13 & 54495 & 168.13 \\
\hline 2612 & 21.89 & 5197 & 21.80 & 31981 & 199.47 & 63637 & 199.47 \\
\hline 3120 & 23.88 & 6207 & 23.88 & 37701 & 226.76 & 75020 & 226.76 \\
\hline 3553 & 25.79 & 7071 & 25.79 & 45042 & 275.51 & 81419 & 275.51 \\
\hline 4140 & 29.39 & 8237 & 29.39 & 40853 & 287.54 & 99201 & 287.54 \\
\hline 6118 & 4221 & 12175 & 42.21 & 56384 & 306.21 & 112198 & 306.21 \\
\hline 2248 & 58.44 & 18403 & 58.44 & 61030 & 305.48 & 121450 & 305.48 \\
\hline 10284 & 68.85 & 20424 & 68.85 & 63006 & 360.30 & 125553 & 360.30 \\
\hline 11733 & 76.61 & 23347 & 76.61 & 68548 & 381.88 & 136401 & 381.88 \\
\hline 15008 & $\$ 8.00$ & 30043 & 86.00 & 76254 & 402.84 & 151736 & 402.84 \\
\hline
\end{tabular}


Tube 2: $\downarrow_{A}=2.252$

\begin{tabular}{|c|c|c|c|c|c|c|c|}
\hline Po & Nuppot4 & $\mathbf{n}_{m}$ & $\mathrm{Nu}_{\mathrm{m}_{\mathrm{m}}} \mathrm{Pp}^{\mathrm{O}}$ & no & Nuppo.4 & $\boldsymbol{n o}_{\mathrm{m}}$ & $\mathrm{Nu}_{\mathrm{m}} / \mathrm{PP}^{\mathrm{O} \cdot 4}$ \\
\hline 1010 & 10.57 & se47 & 19.37 & 8231 & 85.65 & 85173 & 85.65 \\
\hline 1102 & 17.82 & 4201 & 17.82 & $œ 946$ & 84.64 & 37898 & 84.64 \\
\hline 1167 & 20.62 & 4445 & 20.62 & 10475 & 80.88 & 30913 & 85.88 \\
\hline 1386 & 2257 & 8281 & 22.57 & 11058 & $\boldsymbol{e 5 . 8 7}$ & 42135 & ย5.87 \\
\hline 1578 & 25.38 & $\infty 014$ & 25.38 & 11800 & 122.12 & 44965 & 122.12 \\
\hline 1666 & $\mathbf{2 8 . 2 4}$ & 6349 & 2624 & 13072 & 112.52 & 48910 & 112.52 \\
\hline 1784 & 2828 & 6000 & 28.28 & 17898 & 141.14 & 68201 & 141.14 \\
\hline 1893 & 3120 & 7213 & 3120 & 20031 & 150.30 & 78612 & 150.30 \\
\hline 2040 & 29.23 & 7800 & 29.23 & 24953 & 190.78 & 95083 & 190.78 \\
\hline 2112 & 31.54 & 8048 & 31.54 & 28384 & 207.31 & 108156 & 207.31 \\
\hline 2479 & 33.94 & 9446 & 33.84 & 30695 & 2226.60 & 116960 & 226.60 \\
\hline 3105 & 35.55 & 11831 & 35.55 & 32094 & 234.81 & 125722 & 234.81 \\
\hline 3489 & 38.81 & 132096 & 38.81 & 36461 & 258.81 & 138934 & 258.81 \\
\hline 4670 & 42.73 & 17793 & 42.73 & 30850 & 275.61 & 151880 & 275.61 \\
\hline 4636 & 46.10 & 17665 & 46.10 & 44850 & 298.76 & 170000 & 298.76 \\
\hline 5312 & 51.30 & 20240 & 51.30 & 46668 & 371.66 & 177827 & 371.66 \\
\hline 6242 & 57.01 & 23783 & 57.01 & 52576 & 334.81 & 200338 & 334.81 \\
\hline 6766 & 59.64 & 25781 & 59.64 & 50303 & 352.75 & 225970 & 352.75 \\
\hline 8076 & 72.19 & 30773 & 72.19 & 72084 & 393.91 & 276060 & 303.91 \\
\hline 8702 & 70.84 & 33158 & 70.84 & & & & \\
\hline
\end{tabular}

Tube 3: ${\downarrow_{R}}=1.616$

\begin{tabular}{|c|c|c|c|c|c|c|c|}
\hline Ro & Nuplo.4 & $\boldsymbol{P o}_{\mathrm{m}}$ & $\mathrm{Nu}_{\mathrm{m}} / \mathrm{Pr}^{0.4}$ & Po & $\mathrm{Nu} / \mathrm{Pr}^{0.4}$ & $R_{o_{m}}$ & Nu/Pi \\
\hline 1018 & 12.18 & 2785 & 12.16 & 15150 & 81.68 & 44467 & 81.68 \\
\hline 1406 & 16.68 & 3846 & 16.68 & 18335 & 94.96 & 50156 & 94.96 \\
\hline 1650 & 18.10 & 4514 & 18.10 & 21380 & 111.19 & 58513 & 111.18 \\
\hline 1705 & 19.53 & 4911 & 18.53 & 20694 & 129.74 & 64818 & 129.74 \\
\hline 1884 & 21.60 & 6427 & 21.60 & 26758 & 130.05 & 73180 & 130.05 \\
\hline 2464 & 26.81 & 6740 & 26.81. & 29357 & 151.38 & 80300 & 151.38 \\
\hline 2888 & 29.61 & 7901 & 29.61 & 32131 & 165.86 & 87898 & 165.86 \\
\hline
\end{tabular}




\begin{tabular}{|c|c|c|c|c|c|c|c|}
\hline Po & Nuppo.4 & $\boldsymbol{n}_{m}$ & $N_{4}$ PPT & Po & Nuppo.4 & $\mathbf{A o}_{\mathrm{m}}$ & $\mathrm{Nu}_{\mathrm{m}} \mathrm{Pr}^{\mathrm{O} .4}$ \\
\hline $\operatorname{ses} 4$ & 20.07 & $\sec$ & 20.97 & 32020 & 10120 & cerper & 18120 \\
\hline 4145 & 33.89 & 11350 & 83.30 & 38050 & 186.97 & 100030 & 186.87 \\
\hline 674 & 42.33 & 10713 & 42.33 & 39287 & 193.20 & 107473 & 10320 \\
\hline 6758 & 46.65 & 18487 & 46.65 & 41275 & 208.05 & 112912 & 208.05 \\
\hline 8330 & 2202 & 22725 & 6222 & 45330 & 213.30 & 124000 & 213.30 \\
\hline 1008 & 60.93 & 20520 & 00.93 & 51017 & 105.37 & 139561 & 195.37 \\
\hline 10030 & 67.23 & 20003 & 67.23 & 62755 & 284.08 & 171672 & 284.08 \\
\hline 11786 & 7245 & 50242 & 72.45 & 72772 & 200.97 & 189073 & 260.97 \\
\hline
\end{tabular}

Tube 4: $\downarrow_{R}=2.918$

\begin{tabular}{|c|c|c|c|c|c|c|c|}
\hline$R_{\theta}$ & Nu/PP.4 & $R \boldsymbol{e}_{\mathrm{m}}$ & $N u_{n} / P^{0.4}$ & $\mathbf{R o}_{\boldsymbol{\theta}}$ & Nu/Pp.4 & $\mathbf{R}_{\mathrm{m}}$ & $\mathrm{Nu}_{m} / \mathrm{PP}^{\mathrm{O}}$ \\
\hline 1047 & 13.05 & 5170 & 13.05 & 15117 & 117.82 & 74646 & 117.92 \\
\hline 1073 & 16.64 & 5209 & 16.64 & 17308 & 137.08 & 85467 & 137.08 \\
\hline 1374 & 20.83 & 6786 & 20.83 & 19197 & 152.64 & 24795 & 152.64 \\
\hline 1555 & 21.82 & 7678 & 21.82 & 20505 & 160.60 & 101254 & 160.69 \\
\hline 1737 & 23.70 & 8577 & 23.70 & 23395 & 182.61 & 115525 & 182.61 \\
\hline 2362 & 27.45 & 11662 & 27.45 & 25585 & 185.71 & 126337 & 105.71 \\
\hline 2992 & 33.35 & 14775 & 33.35 & 27848 & 206.52 & 137512 & 206.52 \\
\hline 3795 & 38.40 & 18741 & 38.40 & 30562 & 228.60 & 150014 & 228.60 \\
\hline 5203 & 45.94 & 25693 & 45.94 & 33540 & 253.95 & 165622 & 253.95 \\
\hline 6348 & 66.82 & 31348 & 56.82 & 36507 & 268.94 & 180271 & 268.94 \\
\hline 7016 & 62.60 & 34647 & 62.60 & 39830 & 291.00 & 196679 & 291.00 \\
\hline 8274 & 69.04 & 40855 & 69.04 & 42795 & 324.43 & 211322 & 324.43 \\
\hline 8585 & 73.20 & 47331 & 73.29 & 44964 & 336.27 & 222033 & 336.27 \\
\hline 10202 & 75.33 & 50370 & 75.33 & 56168 & 358.25 & 277359 & 358.25 \\
\hline 10059 & 87.60 & 53622 & 87.60 & 62006 & 412.99 & 306188 & 412.90 \\
\hline 11833 & 86.50 & 58434 & 86.50 & 70082 & 434.25 & 346065 & 434.25 \\
\hline 13141 & 100.77 & 64888 & 103.77 & & & & \\
\hline
\end{tabular}


Tube 5: $\downarrow_{A}=2.653$

\begin{tabular}{|c|c|c|c|c|c|c|c|}
\hline Po & Nuphas & $\mathbf{n}_{m}$ & $\mathrm{Nu}_{\mathrm{m}} \mathrm{Pr}^{\mathrm{O}}$ & Re & Nuppo.4 & $\mathbf{R}_{m}$ & $\mathrm{Nu}_{\mathrm{m}} / \mathrm{PP} \mathrm{P}^{\mathrm{N}}$ \\
\hline 1212 & 11.93 & 5410 & 11.93 & 8007 & 56.60 & 41787 & 56.69 \\
\hline 1220 & 13.10 & 8517 & 13.10 & 10707 & 67.41 & 48071 & 67.41 \\
\hline 1317 & 13.68 & 8014 & 13.68 & 11980 & 60.12 & 63096 & 00.12 \\
\hline 1284 & 14.64 & 6675 & 14.84 & 13517 & 77.62 & 60089 & $\pi . \infty$ \\
\hline 1582 & 15.83 & 7014 & 15.86 & 15808 & 80.66 & 70975 & 80.66 \\
\hline 1652 & 17.02 & 7327 & 17.02 & 18185 & 98.82 & 81648 & 96.82 \\
\hline 1774 & 18.11 & 7065 & 18.11 & 19887 & 112.55 & 80280 & 112.55 \\
\hline 1870 & 18.22 & 8438 & 18.22 & 22008 & 112.28 & 100562 & 112.28 \\
\hline 2065 & 19.34 & 9272 & 19.34 & 25255 & 124.58 & 113392 & 124.58 \\
\hline 2000 & 21.25 & 8581 & 21.25 & 27913 & 131.00 & 125325 & 131.00 \\
\hline 2318 & 20.80 & 10405 & 20.80 & 30706 & 141.57 & 137863 & 141.57 \\
\hline 2821 & 22.83 & 12666 & 22.83 & 37819 & 138.07 & 160800 & 138.07 \\
\hline 3241 & 24.62 & 14553 & 24.62 & 33580 & 164.58 & 150766 & 164.58 \\
\hline 4040 & 30.75 & 18180 & $\$ 0.75$ & 40778 & 177.02 & 163087 & 177.02 \\
\hline 5703 & 37.45 & 25607 & 37.45 & 45657 & 237.79 & 204993 & 237.79 \\
\hline 7000 & 44.11 & 31428 & 4.11 & 50015 & 257.00 & 264966 & 257.00 \\
\hline 8362 & 51.58 & 37542 & 51.58 & 67730 & 216.11 & 304093 & 216.11 \\
\hline
\end{tabular}

Tube 6: $\downarrow_{R}=3.29$

\begin{tabular}{|c|c|c|c|c|c|c|c|}
\hline Ro & Nu/Pr 0.4 & $\mathbf{R}_{\mathrm{m}}$ & $\mathrm{Nu}_{\mathrm{m}} / \mathrm{Pr}^{\mathrm{O} .4}$ & Ro & NuPp 0.4 & $\mathbf{P e}_{\mathrm{m}}$ & $N u_{\pi} / P^{0.4}$ \\
\hline 1212 & 11.84 & 6748 & 11.84 & 15779 & 137.54 & 87847 & 137.54 \\
\hline 1443 & 15.79 & 8058 & 15.79 & 17048 & 153.41 & 84915 & 153.41 \\
\hline 1569 & 18.53 & 8733 & 18.53 & 19808 & 185.34 & 110282 & 185.34 \\
\hline 1820 & 19.39 & 10183 & 19.39 & 23259 & 233.87 & 129498 & 233.87 \\
\hline 2064 & 23.05 & 11490 & 23.05 & 25818 & 233.04 & 143740 & 233.04 \\
\hline 2315 & 22.75 & 12889 & 22.75 & 27960 & 212.80 & 155668 & 212.90 \\
\hline 3144 & 21.89 & 17503 & 21.89 & 30818 & 286.69 & 171578 & 286.89 \\
\hline 5050 & 58.08 & 28173 & 58.08 & 33888 & 314.78 & 188671 & 314.78 \\
\hline 6428 & 6232 & 35786 & 62.32 & 36743 & 324.16 & 204568 & 324.16 \\
\hline 7654 & 70.26 & 42616 & 70.28 & 30053 & 379.41 & 217427 & 379.41 \\
\hline 8553 & 68.06 & 53187 & 68.08 & 41801 & 405.20 & 232726 & 405.20 \\
\hline
\end{tabular}




\begin{tabular}{|c|c|c|c|c|c|c|c|}
\hline Po & Nuppos & $\mathbf{R o}_{m}$ & $\mathrm{Nu}_{\mathrm{m}} \mathrm{Pp}^{\mathrm{O}}$ & Ro & Nurpo.4 & $\mathbf{R}_{m}$ & $\mathrm{Nu}_{\mathrm{m}} \mathrm{Pr}^{\mathrm{O} 4}$ \\
\hline 9180 & 7.36 & 81100 & 77.56 & 45058 & 40.44 & 200800 & 440.44 \\
\hline 11538 & ex.01 & 64237 & 94.01 & 52846 & 403.30 & 204223 & 403.30 \\
\hline 12893 & 110.16 & 68090 & 110.16 & 60545 & 474.26 & 337066 & 474.26 \\
\hline 13219 & 125.35 & roses & 125.35 & 84053 & 445.64 & 350000 & 45.64 \\
\hline 14315 & 121.30 & 70702 & 121.30 & & & & \\
\hline
\end{tabular}

Knudsen and Katz (1950): Enhancement factors

\begin{tabular}{|c|c|c|c|c|}
\hline Tube & $\downarrow_{R}$ & $\nu_{f}$ & $\varphi_{1}{ }^{+}$ & $\varphi_{h, \text { max }}$ \\
\hline 1 & 1.99 & 0.533 & 1.082 & 1.54 \\
\hline 2 & 2.252 & 0.433 & 1.703 & 1.80 \\
\hline 3 & 1.616 & 0.619 & 1.682 & 1.26 \\
\hline 4 & 2.918 & 0.333 & 1.822 & 1.90 \\
\hline 5 & 2.653 & 0.371 & 2.37 & 1.20 \\
\hline 6 & 3.29 & 0.296 & 2.385 & 2.40 \\
\hline smooth & 1.692 & 0.615 & 1 & 1 \\
\hline
\end{tabular}

+ Based on $\operatorname{Re}_{m}=50,000$. 
Table A.12

Watkinson et al. (1974)

Enhancement factors for finned tubes

\begin{tabular}{|c|c|c|c|c|}
\hline Tube & $\nabla_{R}$ & 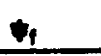 & $S_{\text {minax }}$ & Fin geometry \\
\hline 1 & 2250 & 0.258 & 1.35 & \multirow{7}{*}{$\begin{array}{l}\text { Mith } \\
\text { Epiral } \\
\text { Fins } \\
\text { (HSF) }\end{array}$} \\
\hline 2 & 2.78 & 0.288 & 2.01 & \\
\hline 3 & 2.128 & 0.250 & 1.50 & \\
\hline 4 & 2213 & 0.250 & 1.35 & \\
\hline 5 & 8.050 & 0.286 & 124 & \\
\hline$\theta$ & 2.370 & 0.235 & 1.70 & \\
\hline 18 & 2.817 & 0.070 & - & \\
\hline 11 & 1.434 & 0.267 & 1.62 & \multirow{5}{*}{$\begin{array}{c}\text { High Straight } \\
\text { Fins }\end{array}$} \\
\hline 13 & 1.617 & 0.320 & 1.50 & \\
\hline 14 & 1.888 & 0.308 & 1.60 & \\
\hline 16 & 1.629 & 0.400 & 1.60 & \\
\hline 20 & 1.416 & 0.320 & 1.13 & \\
\hline 15 & 1.045 & 0.333 & 1.87 & \multirow{6}{*}{$\begin{array}{l}\text { Low } \\
\text { Spiral } \\
\text { Fins } \\
\text { (LSF) }\end{array}$} \\
\hline 17 & 1.620 & 0.444 & 1.83 & \\
\hline 19 & 0.887 & 0.333 & 1.57 & \\
\hline 21 & 1.417 & 0.421 & 2.03 & \\
\hline 22 & 1.330 & 0.533 & 1.38 & \\
\hline 23 & 1.421 & 0.521 & - & \\
\hline 10 (smooth) & 1.0 & 1.0 & & 1.0 \\
\hline
\end{tabular}

